\title{
Polar Stratospheric Clouds: Satellite Observations, Processes, and Role in Ozone Depletion
}

\author{
I. Tritscher ${ }^{1}$, M. C. Pitts ${ }^{2}$, L. R. Poole ${ }^{3}$, S. P. Alexander ${ }^{4}$, F. Cairo ${ }^{5}$, M. P. Chipperfield ${ }^{6}$, J.- \\ U. Grooß ${ }^{1}$, M. Höpfner ${ }^{7}$, A. Lambert ${ }^{8}$, B. P. Luo ${ }^{9}$, S. Molleker ${ }^{10}$, A. Orr ${ }^{11}$, R. Salawitch ${ }^{12}$, \\ M. Snels ${ }^{5}$, R. Spang ${ }^{1}$, W. Woiwode ${ }^{7}$, and T. Peter ${ }^{9}$
}

${ }^{1}$ Forschungszentrum Jülich, Institut für Energie und Klimaforschung, Stratosphäre, IEK-7, Jülich, Germany.

${ }^{2}$ NASA Langley Research Center, Hampton, Virginia, USA.

${ }^{3}$ Science Systems and Applications, Inc., Hampton, Virginia, USA.

${ }^{4}$ Australian Antarctic Division, Kingston, TAS, Australia.

${ }^{5}$ Istituto di Scienze dell'Atmosfera e del Clima, Rome, Italy.

${ }^{6}$ School of Earth and Environment, University of Leeds, UK.

${ }^{7}$ Institute of Meteorology and Climate Research, Karlsruhe Institute of Technology, Karlsruhe, Germany.

${ }^{8}$ Jet Propulsion Laboratory, California Institute of Technology, Pasadena, CA, USA.

${ }^{9}$ ETH Zurich, Institute for Atmospheric and Climate Science, Zurich, Switzerland.

${ }^{10}$ Max Planck Institute for Chemistry, Mainz, Germany.

${ }^{11}$ British Antarctic Survey, NERC, Cambridge, UK.

${ }^{12}$ University of Maryland, College Park, MD, USA.

Corresponding authors: Michael Pitts (michael.c.pitts@ nasa.gov)

Ines Tritscher (i.tritscher@fz-juelich.de)

\section{ey Points:}

- We provide a new vortex-wide climatology of polar stratospheric cloud occurrence and composition based on $21^{\text {st }}$ century satellite data.

- We review advances in understanding cloud formation, the role of dynamical processes, and heterogeneous chlorine activation.

- We highlight improvements in techniques for parameterizing polar stratospheric clouds and their effects in global models.

This article has been accepted for publication and undergone full peer review but has not been through the copyediting, typesetting, pagination and proofreading process, which may lead to differences between this version and the Version of Record. Please cite this article as doi: 10.1029/2020RG000702. 


\begin{abstract}
Polar stratospheric clouds (PSCs) play important roles in stratospheric ozone depletion during winter and spring at high latitudes (e.g., the Antarctic ozone hole). PSC particles provide sites for heterogeneous reactions that convert stable chlorine reservoir species to radicals that destroy ozone catalytically. PSCs also prolong ozone depletion by delaying chlorine deactivation through the removal of gas-phase $\mathrm{HNO}_{3}$ and $\mathrm{H}_{2} \mathrm{O}$ by sedimentation of large NAT (nitric acid trihydrate) and ice particles. Contemporary observations by the spaceborne instruments MIPAS (Michelson Interferometer for Passive Atmospheric Sounding), MLS (Microwave Limb Sounder), and CALIOP (Cloud-Aerosol Lidar with Orthogonal Polarization) have provided an unprecedented polar vortex-wide climatological view of PSC occurrence and composition in both hemispheres. These data have spurred advances in our understanding of PSC formation and related dynamical processes, especially the firm evidence of widespread heterogeneous nucleation of both NAT and ice PSC particles, perhaps on nuclei of meteoritic origin. Heterogeneous chlorine activation appears to be well understood. Reaction coefficients on/in liquid droplets have been measured accurately, and while uncertainties remain for reactions on solid NAT and ice particles, they are considered relatively unimportant since under most conditions chlorine activation occurs on/in liquid droplets. There have been notable advances in the ability of chemical transport and chemistry-climate models to reproduce PSC temporal/spatial distributions and composition observed from space. Continued spaceborne PSC observations will facilitate further improvements in the representation of PSC processes in global models and enable more accurate projections of the evolution of polar ozone and the global ozone layer as climate changes.
\end{abstract}

\title{
Plain Language Summary
}

Polar stratospheric clouds (PSCs) occur during winter and early spring in the polar stratosphere, when temperatures are low enough to enable cloud formation despite the extremely dry conditions. Ground-based PSC sightings date back to the late $19^{\text {th }}$ century, but they were little more than a scientific curiosity until the discovery of the Antarctic ozone hole in 1985. Soon thereafter, it was shown that PSCs play a crucial role in converting stable halogen (mainly chlorine) species of anthropogenic origin into reactive gases that rapidly destroy ozone. Considerable progress was made over the next two decades in quantifying these processes through laboratory studies, field campaigns, and limited spaceborne observations. We are now reaping the benefits of new PSC observations over the entire polar regions from three complementary $21^{\text {st }}$ century spaceborne instruments. This paper reviews these instruments and highlights new findings on PSC occurrence and composition. These datasets have also triggered advances in understanding how PSCs form and the influence of atmospheric dynamics, as well as improvements in how detailed cloud processes are approximated in global models. This will ultimately lead to better predictions of how quickly the stratospheric ozone layer will recover from human influence as global climate changes in the future.

This article is protected by copyright. All rights reserved. 


\section{Historical Overview}

There has been a continuing stream of discoveries about polar stratospheric clouds (PSCs) from the first sightings in the late $19^{\text {th }}$ century to contemporary satellite measurements during the $21^{\text {st }}$ century. ${ }^{1}$ While PSCs had been a colorful, fascinating and possibly mysterious phenomenon for the public, and an interesting and challenging research topic for scientists, they remained a meteorological curiosity, occurring naturally and not thought to be harmful in any way. The interest in PSCs changed dramatically after the surprising discovery of the Antarctic ozone hole in the 1980s, when it was hypothesized that the clouds might provide the link between anthropogenic chlorine and polar ozone destruction.

At the time of the discovery of the ozone hole it was known that anthropogenic chlorinecontaining compounds - the chlorofluorocarbons (CFCs) — had been emitted in large amounts into the atmosphere, leading to an almost six-fold increase in stratospheric chlorine loading by 1985 compared to pre-industrial times (WMO, 2018). In the troposphere, CFCs are very stable and long-lived. It was known that they are destroyed by photolysis in the stratosphere, and that the released chlorine could deplete ozone through gas-phase reactions (Molina and Rowland, 1974). However, this ozone depletion would occur primarily under sunlit conditions at low latitudes and would mainly affect the upper stratosphere $(30-50 \mathrm{~km})$, not the lower stratosphere, where the bulk of the ozone resides. Thus, the overall depletion was expected to be 5-10\%. If this were correct, how could reactions involving chlorine be responsible for the ozone hole, under conditions with little insolation, destroying a major portion of the Antarctic ozone layer?

The hypothesis of PSCs' role in polar ozone destruction was twofold: first, that PSCs would host heterogeneous chemical reactions on their particle surfaces to activate chlorine, i.e. transforming it from relatively inert forms into ozone-destroying forms; second, that PSC particles would act as scavengers of reactive nitrogen, which can then no longer serve to deactivate the chlorine. Because PSCs occur at altitudes comprising the bulk of the ozone layer, the activated chlorine can destroy a significant amount of ozone. Polar vortex-wide observations by the contemporary spaceborne instruments MIPAS (Michelson Interferometer for Passive Atmospheric Sounding), MLS (Microwave Limb Sounder), and CALIOP (Cloud-Aerosol Lidar with Orthogonal Polarization), a central part of this review paper, have brought about a comprehensive and clearer understanding of PSC spatial and temporal distributions, their conditions of existence, and the processes through which they impact polar ozone.

\subsection{Pre-satellite Era}

In the years 1883-1886, the readers of Nature had the pleasure of following a vivid controversy between those who had observed an apparently new type of cloud and "nonobservers" or non-believers (Dieterichs, 1950). What had happened? Clouds of peculiar shapes in brilliant colors had been observed all over England: "cirrocumulus fleeces became lighted up with a pink and then with a deep red colour... A very peculiar greenish and white opalescent haze ... and shone as if with a light of its own..." (Russell, 1883). What the readers of Nature did not know was that a Norwegian meteorologist had already compared the clouds with the colors of mother-of-pearl (Reusch, 1882). About a decade later, the terms "mother-of-pearl clouds" or "nacreous clouds" were suggested as scientific terms (Mohn, 1895). The acronym "PSCs" was

${ }^{1}$ There are many important and detailed steps in the discovery and understanding of PSCs that are not reviewed here in their entirety. Supplement 1 provides a chronological list of more than fifty of the more important developments, and some citations missing here might be mentioned in this supplement.

This article is protected by copyright. All rights reserved. 
introduced only with the advent of satellite measurements (McCormick et al., 1981; 1982). Indeed, the existence of the stratosphere itself was not known until 1902, and only thereafter it became clear that the clouds we now call PSCs are in the stratosphere. In a series of papers, the Norwegian mathematician and astrophysicist Carl Størmer determined their altitude, particle size, and temperature (Størmer, 1929; 1932a,b; 1935). Figure 1 shows: (a) a photograph of PSCs by Størmer from January 1929; (b) his method of determining their altitude; and (c) a modern photograph of PSCs over Finland in February 2017. Before the advent of photography, there were only sketches and paintings, possibly such as Munch's "The Scream" (Figure 1d; Fikke et al., 2017; Prata et al., 2018). The clouds seemed to remain motionless at high altitude despite strong Föhn winds on the ground (Størmer, 1948), which was explained soon thereafter by Dieterichs (1950) in terms of the formation and trapping of the clouds by lee waves downstream of the Scandinavian mountain range. He interpreted moving clouds as break-off lee wave clouds drifting with the overall air flow, while small motions could simply be related to changes in the quasi-stationary wave system, ideas that were confirmed quantitatively more than 50 years later by high-resolution meteorological models (Dhaniyala et al., 2002; Fueglistaler et al., 2002a).

A global stratospheric aerosol layer was discovered in 1960 when Christian Junge launched balloons from Sioux Falls (South Dakota, USA) and Hyderabad (India) and found submicron particles in a layer between the tropopause and about $35 \mathrm{~km}$ altitude. These looked like liquid droplets composed of sulfuric acid and water, formed by the chemical transformation of sulfur-containing gases. This led to the notion of "A World-wide Stratospheric Aerosol Layer" (Junge et al., 1961a, b).

A report on PSC sightings (Stanford and Davis, 1974) listed 156 observations during the century from 1870 to 1972 . Thus, observational evidence was growing, but there were a number of open questions by the dawn of the satellite era (see Toon and Farlow, 1981): Do these clouds consist purely of water ice? Do they interact with the stratospheric $\mathrm{H}_{2} \mathrm{SO}_{4}-\mathrm{H}_{2} \mathrm{O}$ particles in the Junge aerosol layer? Does ice nucleate in these preexisting droplets, and if so, how? Is there enough water vapor and are temperatures low enough, e.g. in the crests of lee waves, to trigger formation of the clouds?

\subsection{Early Satellite Measurements}

For PSCs, the satellite era begins with SAM II (Stratospheric Aerosol Measurement II), a single channel (1- $\mu \mathrm{m}$ wavelength) solar occultation photometer onboard the Nimbus 7 satellite that collected data from October 1978 to December 1993. SAM II measured vertical profiles of aerosol and cloud extinction at latitudes ranging from about $64^{\circ}$ at the solstices to about $80^{\circ}$ at the equinoxes in both hemispheres, making it ideal for PSC observations. The initial SAM II results from 1978/79 were intriguing, showing wintertime PSCs to be strongly associated with low temperature and much more prevalent in the Antarctic than in the Arctic, increasing the Antarctic stratospheric optical depth in a distinct manner for a period of about three months (McCormick et al., 1981). As the data record lengthened, it revealed a high level of annual regularity in Antarctic PSC activity, with sharp increases in stratospheric optical depth beginning each June and ending in September (McCormick et al., 1993). Similar to a heartbeat, the maxima were followed by peculiar minima in October, possibly a cleansing of the air due to scavenging of aerosol by sedimenting large PSC particles. The Arctic showed a similar annual cycle, though with much less pronounced wintertime maxima in optical depth and no end-of-winter minima,

This article is protected by copyright. All rights reserved. 
with high interannual variability and more obvious perturbations due to volcanic eruptions, such as that of El Chichón in 1982.

SAM II was a game changer in terms of PSCs, transforming our observational database from sporadic, weather-dependent glimpses made from the surface into a multi-year climatology. The instrument showed excellent sensitivity, with typical extinction enhancements under PSC conditions of 1-2 orders of magnitude above background. While this was great progress, little attention was paid to the fact that this extinction increase was actually relatively small compared to that expected from ice clouds, and that the extinction increase seemed to persist up to several degrees above the frost point $\left(T_{\text {ice }}\right)$. Were the observed extinctions actually consistent with pure ice PSCs that were confined to small cold regions scattered across the satellite field-of-view? Or were the faint events hinting that there were broad-scale, optically thinner PSCs whose composition (see Table 1) was something other than ice? These questions were asked and answered only after the discovery of the ozone hole (Toon et al., 1986).

\section{Table 1: PSC Composition}

In this review, "PSC composition" is used as an umbrella term to classify PSC chemical components, waters of crystallization, particle phase states, and states of external and internal mixing.

(i) Chemical species - PSC chemical species include stratospheric sulfuric acid aerosols ( $\mathrm{SSA}, \mathrm{H}_{2} \mathrm{SO}_{4}-\mathrm{H}_{2} \mathrm{O}$ ) or supercooled ternary solution (STS, $\mathrm{H}_{2} \mathrm{SO}_{4}-\mathrm{HNO}_{3}-\mathrm{H}_{2} \mathrm{O}$ ) droplets, nitric acid trihydrate (NAT, $\mathrm{HNO}_{3} \cdot 3 \mathrm{H}_{2} \mathrm{O}$ ), other hydrates of $\mathrm{HNO}_{3}$ or $\mathrm{H}_{2} \mathrm{SO}_{4}, \mathrm{H}_{2} \mathrm{O}$ ice, or mixed ensembles of any of these. There are caveats with respect to chemical speciation in the context of remote sensing. MIPAS detects the IR spectral signature of small (radius $<2 \mu \mathrm{m}$ ) NAT particles, but most other remote observations do not provide direct chemical information. However, based on our theoretical and laboratorybased understanding of stratospheric species, PSC chemical speciation can be inferred from lidar backscatter data; e.g. only $\mathrm{H}_{2} \mathrm{O}$ ice can explain large backscatter magnitudes, and only SSA or STS droplets can explain non-depolarized backscsatter signals. This is the basis for the PSC composition classes used throughout this paper.

(ii) Waters of crystallization - Laboratory experiments have shown crystalline $\mathrm{HNO}_{3}$ hydrates, in particular NAT and nitric acid dihydrate (NAD, $\mathrm{HNO}_{3} \cdot 2 \mathrm{H}_{2} \mathrm{O}$ ), to be possible constituents of solid phase PSC particles. The stratospheric stability regions of NAT and NAD overlap, and both have been shown to exist in different (so-called $\alpha$ and $\beta$ ) forms and sulfuric acid tetrahydrate $\left(\mathrm{SAT}, \mathrm{H}_{2} \mathrm{SO}_{4}{ }^{\bullet} 4 \mathrm{H}_{2} \mathrm{O}\right)$ to be possible constituents of solid phase PSC particles. However, only NAT has been detected by balloonborne mass spectrometry, and only $\beta$-NAT has been identified by satelliteborne IR spectrometry (MIPAS). Crystalline hydrates of $\mathrm{H}_{2} \mathrm{SO}_{4}$ might also exist in the stratosphere, but laboratory experiments have shown their formation to be kinetically suppressed. Therefore, in this paper, while NAD and other hydrates cannot be completely excluded under stratospheric conditions, their existence is not likely, and we use "NAT" as an umbrella term for $\mathrm{HNO}_{3}$ containing hydrates.

(iii) Phase state - The phase state of PSC particles can be inferred from polarizationsensitive lidar measurements due to the fact that non-spherical crystals depolarize the

This article is protected by copyright. All rights reserved. 


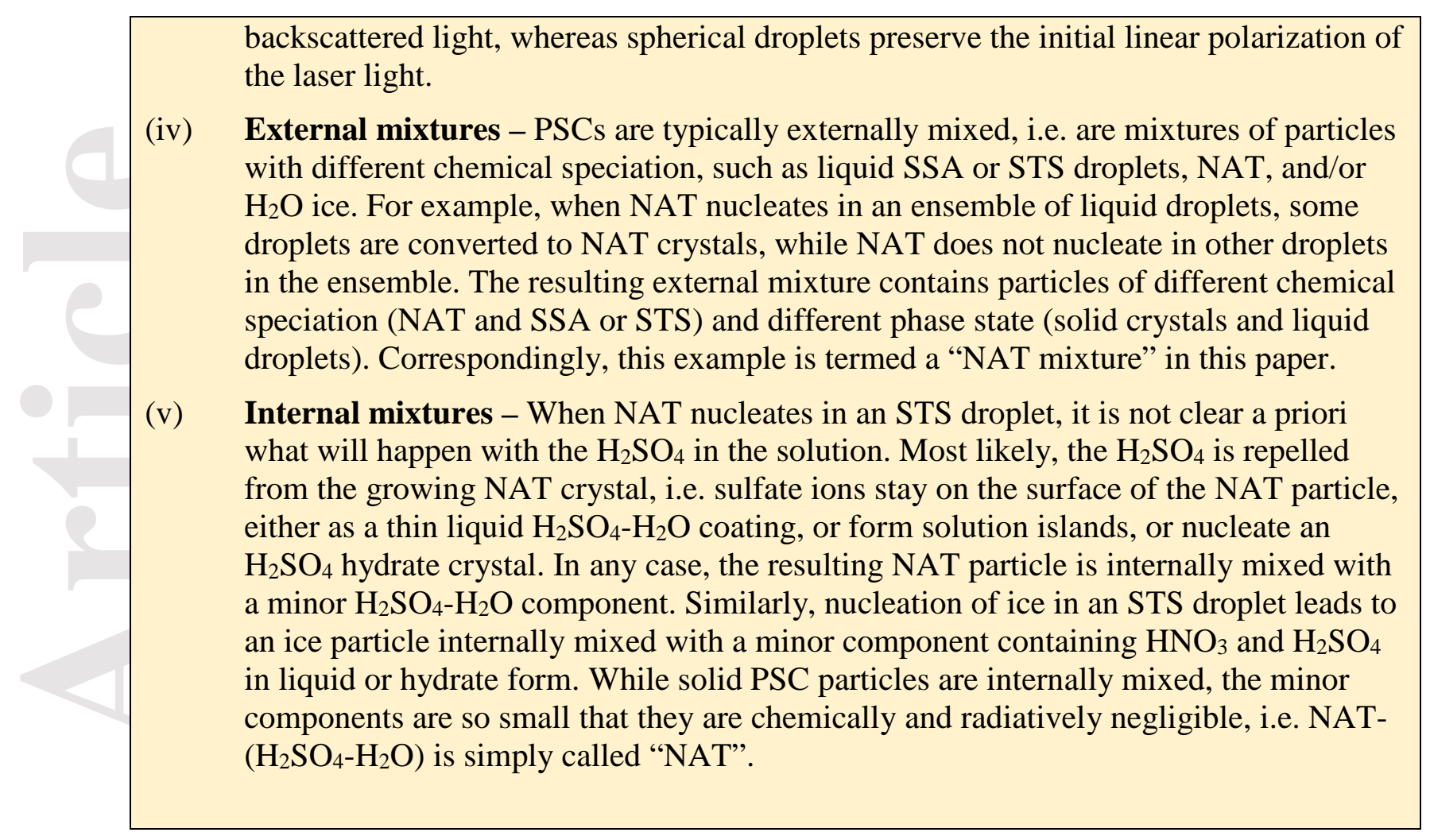

\subsection{The years after discovery of the ozone hole}

In 1985, Joe Farman and colleagues (Farman et al., 1985) reported a discovery that caused disbelief among experts and anxiety in the broader public: strong ozone losses over Antarctica, recurring each September, a phoneomenon later termed the "ozone hole." Farman et al. explained that the ozone loss was caused by anthropogenic chlorine, which was first emitted as CFCs, then was transformed in the atmosphere into active chlorine that destroyed ozone above Antarctica. Later it became clear that their basic point, namely anthropogenic chlorine as cause of this phenomenon, was correct, even though the chemical mechanism they suggested was not. The correct mechanism was related, at least in part, to heterogeneous chemical reactions on the surfaces of PSC particles. In a publication only one year after Farman's discovery, Susan Solomon and coworkers showed that homogeneous (gas phase) chemistry could not explain the observed ozone loss. They argued that PSCs are a unique feature of the extremely cold conditions in the polar lower stratosphere, presumably providing a reaction site for heterogeneous chemical reactions. Solomon et al. (1986) specifically suggested the heterogeneous reaction between $\mathrm{HCl}$ and $\mathrm{ClONO}_{2}$ activated the chlorine, which would then destroy ozone. At the time, this was a bold hypothesis, as it demanded two relatively inert chlorine reservoir gases, $\mathrm{HCl}$ and $\mathrm{ClONO}_{2}$, to react with each other, catalyzed by the ice surfaces in PSCs. In the end, this hypothesis would be fully confirmed.

Heterogeneous chemistry had to this point been neglected, or rather was perceived as a nuisance in work on chemical kinetics, as results for homogeneous gas phase reactions could be compromised by heterogeneous reactions on reactor walls. The suggestion by Solomon et al. (1986) released a wave of investigations into heterogeneous chemistry spanning the period from then until 2000. In a number of laboratories, the reaction kinetics of $\mathrm{ClONO}_{2}, \mathrm{HOCl}$, and $\mathrm{N}_{2} \mathrm{O}_{5}$

This article is protected by copyright. All rights reserved. 
were measured on solid stratospheric materials, first on $\mathrm{H}_{2} \mathrm{O}$ ice, then also on NAT and other hydrates of $\mathrm{HNO}_{3}$ and $\mathrm{H}_{2} \mathrm{SO}_{4}$ with and without $\mathrm{HCl}$-doping (e.g., Hanson and Ravishankara, 1991; Abbatt and Molina, 1992a,b). Later, measurements on $\mathrm{H}_{2} \mathrm{SO}_{4}-\mathrm{H}_{2} \mathrm{O}$ and $\mathrm{H}_{2} \mathrm{SO}_{4}-\mathrm{HNO}_{3}-\mathrm{H}_{2} \mathrm{O}$ solutions were added (e.g., Zhang et al., 1994; Elrod et al., 1995; Ravishankara and Hanson, 1996).

In addition to direct heterogeneous chlorine activation, there are also PSC microphysical processes that promote polar chlorine activation and ozone loss. Based on thermodynamic considerations, Toon et al. (1986) argued that $\mathrm{HNO}_{3}$ hydrates, among them NAT, existed at temperatures above $T_{\text {ice }}$ and would lead to optically thinner PSCs (by condensing only some parts per billion of stratospheric $\mathrm{HNO}_{3}$ versus some parts per million of stratospheric $\mathrm{H}_{2} \mathrm{O}$ in the case of ice). This could explain why PSCs observed by SAM II seemed too optically thin and occurred at too high temperatures to be ice. Further, this would lead to significant denoxification (conversion of $\mathrm{NO}_{\mathrm{x}}$ to $\mathrm{HNO}_{3}$ ) or even denitrification (removal of $\mathrm{HNO}_{3}$ from the stratosphere by gravitational settling of hydrate particles), which would disable the deactivation of chlorine by $\mathrm{NO}_{x}$. Using chemical box modeling, Crutzen and Arnold (1986) showed that this would indeed enhance chlorine activation. All these processes, heterogeneous chlorine activation on PSC surfaces, denoxification, and denitrification, as well as dehydration by sedimenting ice particles, were later shown to affect polar ozone depletion (see Section 5).

While the long limb-viewing path of SAM II could not provide conclusive evidence for two classes of PSC particles, Arctic airborne lidar measurements revealed the signature of small

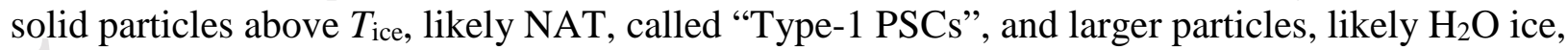
called "Type-2 PSCs" (Poole et al., 1988; Poole and McCormick, 1988). Subsequent mass spectrometric laboratory measurements of NAT vapor pressures $\left(\mathrm{HNO}_{3}\right.$ and $\left.\mathrm{H}_{2} \mathrm{O}\right)$ proved that under stratospheric conditions NAT could indeed exist at temperatures up to $7 \mathrm{~K}$ above $T_{\text {ice }}$ (Hanson and Mauersberger, 1988). The verification of NAT in PSCs in the atmosphere would occur some twelve years later with balloon-borne mass spectrometry (Voigt et al., 2000). A combined analysis by Toon et al. (1990) of lidar backscatter ratios and depolarization ratios revealed that there are actually three PSC types: Type- 2 for ice, Type-1a for non-spherical NAT particles (depolarizing), and a third "Type-1b", which was of unknown composition at that time, but basically consisted of spherical or almost spherical (non-depolarizing) particles with radii $\sim 0.5 \mu \mathrm{m}$. In situ airborne measurements of size distributions and particle growth in Arctic PSCs as function of temperature above $T_{\text {ice }}$ (Dye et al., 1992) coupled with thermodynamic modeling of electrolytic $\mathrm{HNO}_{3}-\mathrm{H}_{2} \mathrm{SO}_{4}-\mathrm{H}_{2} \mathrm{O}$ solutions (Carslaw et al., 1994) provided evidence that the "Type-1b" particles were liquid STS droplets. The identification of Type-1b as liquid STS droplets was supported by laboratory experiments showing that stratospheric binary and ternary solutions remained liquid to temperatures about $4 \mathrm{~K}$ below $T_{\text {ice, }}$, where they crystallized after ice nucleated homogeneously (Koop et al., 1995). The classification of PSCs in three pure categories as Type-1a, Type-1b and Type-2, though still well-known, has become outdated and is no longer recommended, since these "types" are known to be NAT, STS, and ice.

\subsection{The golden era of satellite PSC observations}

While SAM II provided an unprecedented PSC climatology for the Arctic and the Antarctic, its measurements on any particular day were restricted to the latitude at which the solar occultation occurred (i.e., they did not span the polar vortex) and they did not help to distinguish PSC composition. The SAM II climatology was later extended and improved using

This article is protected by copyright. All rights reserved. 
data from the solar occultation instruments Polar Ozone and Aerosol Measurement (POAM) II and III and Stratospheric Aerosol and Gas Experiment (SAGE) II (Fromm et al., 1997, 1999, 2003). Related papers by Strawa et al. (2002) and Poole et al. (2003) showed that using twowavelength solar occultation extinction data gave additional, but again spatially limited information on PSC composition. Valuable information on PSC composition and PSC-related processes, e.g. denitrification and dehydration, was also provided by analyses of data from the Improved Stratospheric and Mesospheric Sounder (ISAMS), Cryogenic Limb Array EtaIon Spectrometer (CLAES), and a first-generation MLS onboard the NASA Upper Atmospheric Research Satellite (e.g., Taylor et al., 1994, Massie et al. 1997; Santee et al., 1995).

We are now reaping the benefits of a "golden age" of long-term, polar-vortex wide PSC observations by three spaceborne instruments that provide fundamentally different, but synergistic information on PSCs. MIPAS, which operated onboard the European Space Agency Envisat satellite from July 2002 to April 2012, collected IR spectra in a limb-viewing mode, from which PSCs can be detected and their composition identified NAT spectroscopically (Höpfner, 2004; Spang et al., 2005). A second-generation MLS, which has been operating since 2004 onboard the NASA Aura satellite, detects spectroscopically the presence of many gaseous species in its field of view, including the PSC precursor gases $\mathrm{HNO}_{3}$ and $\mathrm{H}_{2} \mathrm{O}$ (Lambert et al., 2012). CALIOP, which has been operating since 2006 onboard the joint NASA-CNES environmental satellite CALIPSO (Cloud-Aerosol Lidar and Infrared Pathfinder Satellite Observations), measures backscatter from atmospheric aerosols and clouds, including PSCs (Pitts et al., 2007). CALIOP provides very high spatial and vertical resolution profiles of PSCs as well as indirect information on PSC composition by the presence or absence of changes in the initial polarization state of the laser beam. Rigorous checks and comparisons against data from MIPAS and MLS have enabled the verification and correct implementation of the CALIOP PSC composition classification scheme, and the combination of data from the three sources have fundamentally improved our quantitative knowledge of PSC spatial and temporal distributions and composition.

Section 2 of this paper deals extensively with results from MIPAS, MLS and CALIOP and the resulting better understanding of PSC distributions and composition. Section 3 details our understanding of nucleation pathways of solid PSC particles, where significant progress has been made in the wake of results from the spaceborne instruments, in particular concerning heterogeneous nucleation processes. Section 4 reviews the dynamical forcing of PSCs on different spatial scales, highlighting how small-scale dynamical motions help overcome solid PSC formation barriers and strongly influence the size and number density of the resultant PSC particles. Section 5 reviews our present understanding of heterogeneous chemistry, denitrification, and dehydration by PSCs and their effect on ozone, highlighting the differences between the Arctic and Antarctic polar vortices. Finally, Section 6 describes how PSCs are implemented in present-day chemical transport models and chemistry-climate models, distinguishing between resolved processes versus those for which parameterizations are needed.

\section{PSC Spatial and Temporal Distributions and Composition}

This section will describe the spaceborne CALIOP, MIPAS, and MLS measurements in terms of PSC-related products and compare their theoretical sensitivities to STS and NAT PSC particles. We will discuss how differences in spatial resolution affect intercomparisons of the data and show that the datasets are consistent in terms of PSC composition for scenes that are

This article is protected by copyright. All rights reserved. 
spatially homogeneous. We will then summarize findings on PSC spatial and temporal distributions and composition based on the high spatial resolution CALIOP data record from 2006-2018. We will also explore multi-decadal trends in PSC occurrence by comparing CALIOP data to several long-term polar ground-based lidar data records and the SAM II solar occultation PSC record from 1978-1989.

\subsection{Contemporary PSC Datasets}

\subsubsection{Spaceborne Measurements}

\subsubsection{CALIOP}

CALIOP is a near-nadir viewing two-wavelength polarization-sensitive lidar (532 and $1064 \mathrm{~nm}$ ), with the 532-nm signal separated into orthogonally polarized components (parallel and perpendicular to the polarization plane of the transmitted laser pulse). CALIPSO flew in a 705-km altitude, $98^{\circ}$ inclination orbit as part of the NASA A-train satellite constellation (Stephens et al., 2002), along with the Aqua, Aura, CloudSat, and Orbiting Carbon Observatory2 (OCO-2) satellites, until September 2018, when CALIPSO and CloudSat were maneuvered to fly in formation in a lower $688-\mathrm{km}$ altitude, $98^{\circ}$ inclination orbit. CALIOP has been collecting data nearly continuously from $82^{\circ} \mathrm{S}$ to $82^{\circ} \mathrm{N}$ latitude along $14-15$ orbits per day from mid-June 2006 to the present (Winker et al., 2009). CALIOP PSC studies (Pitts et al., 2007; 2009; 2011; $2013 ; 2018)$ are based on night-time-only measurements of 532-nm parallel $\left(\beta_{\|}\right)$and perpendicular $\left(\beta_{\perp}\right)$ backscatter coefficients assembled on a uniform high spatial resolution grid (5-km along-track $\times 180$ - $\mathrm{m}$ vertical) over altitudes from $8.4-30 \mathrm{~km}$. PSCs are detected as statistical outliers above the existing background stratospheric aerosol in either $\beta_{\perp}$ or in $R_{532}$, which is the ratio of measured total $\left(\beta_{\|}+\beta_{\perp}\right)$ backscatter to molecular backscatter at $532 \mathrm{~nm}$. Successive horizontal averaging $(5,15,45$, and $135 \mathrm{~km})$ ensures that strongly scattering PSCs are found at the finest possible resolution, while enabling the detection of more tenuous PSCs through additional averaging. Because of low signal-to-noise ratio (SNR) at typical stratospheric signal levels, the 1064-nm data have not been used quantitatively in PSC studies to date except to confirm the presence of wave-ice PSCs (Pitts et al., 2011).

Based on a comparison of CALIOP observations of $R_{532}$ and $\beta_{\perp}$ with optical calculations of these quantities for temperature-dependent non-equilibrium mixtures of liquid droplets and NAT or ice spheroids, CALIOP PSCs are separated into three major composition classes: STS; liquid-NAT mixtures ("NAT mixtures"), and liquid-ice mixtures ("ice"). An aspect (diameter-tolength) ratio $\varepsilon=0.9$ is assumed for the NAT and ice spheroids since Engel et al. (2013) found that it produced the best agreement between modeled and observed CALIOP particulate depolarization ratios. Measurements with enhanced $R_{532}$ but no detectable enhancement in $\beta_{\perp}$ are classified as STS (Pitts et al., 2009), representing either pure STS or mixtures of STS and low number densities of NAT or ice particles with undetectable $\beta_{\perp}$. Measurements with detectable enhancements in $\beta_{\perp}$ contain non-spherical (solid) particles and are classified as NAT mixtures or ice. If $T>T_{\mathrm{STS}}$ (STS equilibrium temperature, $\cong T_{\text {ice }}+4 \mathrm{~K}$; Carslaw et al., 1995), the NAT mixtures are SSA and NAT, whereas if $T<T_{\mathrm{STS}}$, the NAT mixtures are STS and NAT. Enhanced NAT mixtures is a sub-class of NAT mixtures with $R_{532}>2$ and $\beta_{\perp}>2 \times 10^{-5} \mathrm{~km}^{-1} \mathrm{sr}^{-1}$, representing those with higher number densities of NAT particles, and corresponding roughly to mixtures with NAT particle radius $\left(r_{\mathrm{NAT}}\right)<3 \mu \mathrm{m}$ and NAT volume density (VD) $>1.0 \mu \mathrm{m}^{3} \mathrm{~cm}^{-3}$

This article is protected by copyright. All rights reserved. 
(Pitts et al., 2018). Wave ice is a sub-class of ice with $R_{532}>50$, representing very high number density ice clouds typical of mountain-wave-induced PSCs.

As an example, Figure 2a shows the distribution of CALIOP Antarctic PSC measurements between 10-18 July 2008 in the $\beta_{\perp}$ vs. $R_{532}$ coordinate system, with PSC composition class/sub-class boundaries. Figure $2 \mathrm{~b}$ shows optical calculations presented in the same reference frame assuming $50 \mathrm{hPa}$ atmospheric pressure, $5 \mathrm{ppmv} \mathrm{H}_{2} \mathrm{O}$, and 3 ppbv $\mathrm{HNO}_{3}$, with instrument noise, which is predominantly shot noise, simulated using the approach of Tritscher et al. (2019).

The CALIOP v2 PSC algorithm (Pitts et al., 2018) also incorporates a retrieval of 532-nm particulate backscatter coefficient which is used to estimate cloud microphysical quantities, namely surface area density (SAD) and VD. These can be compared with measurements from other sensors and used in global models (cf. Section 6). The high spatial resolution of the CALIOP v2 PSC product is illustrated in Figure 3, which presents curtains from one CALIPSO orbit crossing the Antarctic on 17 July 2008 of (a) $R_{532}$, (b) $\beta_{\perp}$, (c) PSC presence and inferred composition, illustrating the spatial inhomogeneity that is often observed, and (d) estimated SAD.

\subsubsection{MIPAS}

MIPAS is a limb-scanning Michelson interferometer that operated onboard the Envisat satellite from July 2002 to April 2012, measuring infrared spectra in the 4-15 $\mu \mathrm{m}$ wavelength range (Fischer et al., 2008). Envisat flew in a sun synchronous ( $98.4^{\circ}$ inclination) orbit, which coupled with additional poleward tilt of the primary MIPAS mirror, allowed measurement coverage almost all the way to both poles (typically to $87^{\circ} \mathrm{S}$ and $89^{\circ} \mathrm{N}$ ). MIPAS measured with very high spectral resolution $\left(0.025 \mathrm{~cm}^{-1}\right)$ from $2002-2004$, after which the resolution was reduced to $0.0625 \mathrm{~cm}^{-1}$ due to technical problems with the interferometer. The MIPAS field of view (FOV) has a vertical full-width half-maximum of $\sim 3 \mathrm{~km}$ and a cross-track extent of $\sim 30$ $\mathrm{km}$, and the MIPAS limb path in the tangent height layer is approximately $400 \mathrm{~km}$.

Infrared spectra measured in the lower stratosphere during polar winters are strongly influenced by the presence of PSC particles. Spang and Remedios (2003) developed a two colorratio (2-CR) approach to determine PSC composition from spectra measured by CRISTA (Cryogenic Infrared Spectrometers and Telescopes for the Atmosphere), which was later refined and applied to MIPAS spectra by Spang et al. (2005). Through comparison with simulated spectra based on laboratory measurements, $\beta$-NAT, i.e. the stable phase of NAT (Koehler et al., 1992), STS, and ice were positively identified in MIPAS spectra (Höpfner et al., 2006a). The MIPAS cloud index (CI), defined as the ratio of the mean spectral intensities in the 788.2$796.2 \mathrm{~cm}^{-1}$ and $832.0-834.4 \mathrm{~cm}^{-1}$ spectral intervals, is sensitive to PSC optical thickness and extinction. Further, the MIPAS NAT index (NI), defined as the ratio of the mean spectral intensities in the $819-821 \mathrm{~cm}^{-1}$ and $788.2-796.2 \mathrm{~cm}^{-1}$ spectral intervals, is sensitive to emissions from NAT particles smaller than about $2-3 \mu \mathrm{m}$ in radius. Recently, Spang et al. (2016) generalized this approach by including five brightness temperature differences (BTD) in addition to CI and NI. This new approach is based on an extensive database of more than 600,000 modelled spectra (Spang et al., 2012) for homogenous layers having a single PSC composition

This article is protected by copyright. All rights reserved. 
(STS, NAT, or ice), with variable microphysical (VD, median particle radius) and macrophysical (horizontal and vertical extent) properties.

The sampling volume of MIPAS $(\sim 3 \mathrm{~km}$ vertical $\times 30 \mathrm{~km}$ cross track $\times 400 \mathrm{~km}$ along the instrument line of sight) is significantly larger than that of CALIOP, and it is unlikely that a single PSC composition would fill this entire volume. The appearance of the peak around 820 $\mathrm{cm}^{-1}$ in MIPAS spectra (Figure $\left.4 \mathrm{a}\right)$ is attributed to small NAT particles $(<2-3 \mu \mathrm{m}$ radius) and provides confidence that these particles are present in the sampling volume, but offers no quantitative information on how much of the measurement volume is filled by NAT. The MIPAS operational PSC composition classification algorithm uses a simple Bayesian probabilistic scheme based on a combination of multiple 2D probability density functions of cloud index, NAT index, and brightness temperature differences to attribute a probability, $P_{\mathrm{j}},(\mathrm{j}=1,2,3)$ that each of the three PSC particle compositions (STS, NAT, or ice) contributes to a measured cloud spectrum. If any $P_{\mathrm{j}}>50 \%$, that particular PSC composition is presumed predominant in the spectrum. More specifically, the major PSC compositions are mapped into five MIPAS PSC categories: (1) ice $\equiv P_{\text {ice }}>50 \%$; (2) NAT $\equiv P_{\mathrm{NAT}}>50 \%$, indicating the presence of small NAT particles; (3) STSmix $\equiv P_{\text {STS }}>50 \%$, indicating the presence of either pure STS or STS with embedded larger NAT particles ( $>2-3 \mu \mathrm{m}$ radius) that cannot be distinguished using the current MIPAS algorithm; (4) NAT_STS, where both $P_{\text {NAT }}$ and $P_{\text {STS }}$ are between 40-50\%; and (5) "unknown" if $P<40 \%$ for all three compositions. The MIPAS classes should be interpreted as the most likely PSC particle composition(s) present within the sampling volume but does not exclude the presence of other compositions. Table 2 provides a summary comparison of the CALIOP and MIPAS measurement approaches and composition class definitions.

Figure 4 (a-d) presents examples of MIPAS measurements from four different limb scans to illustrate the unique spectral fingerprints produced by the various PSC compositions. Panel (a) shows spectra measured over the Antarctic on 17 July 2008, with the characteristic spectral signature of NAT highlighted by the red arrow near $820 \mathrm{~cm}^{-1}$. Panel (b) shows spectra measured over the Arctic on 11 December 2011, with the modified step-like feature near $820 \mathrm{~cm}^{-1}$ instead of the sharper peak seen in Panel (a). Based on an analysis of airborne observations, Woiwode et al. (2016) proposed that this modified NAT feature is an indicator of the presence of highly aspherical $\beta$-NAT particles (see also Section 3.2.1). Recently, a simple detection method sensitive to this modified step-like feature and considering also the distinct scattering pattern towards higher wave numbers was successfully applied for vortex-wide detection of large aspherical $\beta$-NAT in the Arctic winter 2011/12 (Woiwode et al., 2019). However, this modified NAT feature (hence the presence of large aspherical NAT particles) is not considered in the current MIPAS PSC composition algorithm. A third example of MIPAS limb spectra in Panel (c) from 16 January 2010 illustrates the characteristic spectral shape (gradient from 830 to $940 \mathrm{~cm}^{-1}$ ) produced by ice PSCs. As highlighted by the blue arrows, the minimum in the absorption spectrum around $960 \mathrm{~cm}^{-1}$ creates a significant reduction in brightness temperature compared to the $830 \mathrm{~cm}^{-1}$ atmospheric window region (e.g. Spang et al., 2016). Panel (d) shows STS spectra from 17 July 2008 that are characterized by a relatively constant baseline compared to the typical ice spectra in panel (c) and no distinct spectral features. Trace gas emissions are still clearly visible in the STS spectra, indicating the relatively small optical thickness of the clouds. The different spectral behavior in the IR for different PSC compositions and the sensitivity of the spectra to particle size makes the MIPAS measurements extremely valuable, in particular in conjunction with the high spatial resolution CALIOP measurements. MIPAS measurements

This article is protected by copyright. All rights reserved. 
provide the spectroscopic underpinning for the PSC composition inferred from the CALIOP data.

Table 2. CALIOP and MIPAS PSC composition class definitions.

\section{CALIOP PSC Composition Classes}

Measurement Technique: Nadir-viewing, polarization-sensitive lidar (532-nm backscatter)

Measurement Volume: $180 \mathrm{~m} \times 100 \mathrm{~m} \times 5 \mathrm{~km}$

Classification Approach: Inferred by comparison of measured $\beta_{\perp}$ and $R_{532}$ with theoretical optical calculations

\begin{tabular}{|c|c|}
\hline STS & $\begin{array}{l}\text { Pure STS or external mixtures of STS and low number densities of NAT or ice } \\
\text { particles with undetectable } \beta_{\perp}\end{array}$ \\
\hline NAT Mixtures & $\begin{array}{l}\text { External mixtures of low number densities of NAT particles with SSA or STS } \\
\text { droplets }\end{array}$ \\
\hline Enhanced NAT Mixtures & $\begin{array}{l}\text { Sub-class of NAT mixtures with } R_{532}>2 \text { and } \beta_{\perp}>2 \times 10^{-5} \mathrm{~km}^{-1} \mathrm{sr}^{-1} \text { representing } \\
\text { those with higher number densities of NAT particles. These observations } \\
\text { correspond roughly to those NAT mixtures with } \mathrm{r}_{\mathrm{NAT}}<3 \mu \mathrm{m} \text { and NAT VD }>1.0 \\
\mu \mathrm{m}^{3} \mathrm{~cm}^{-3}\end{array}$ \\
\hline Ice & External mixture of ice particles and STS droplets \\
\hline Wave Ice & $\begin{array}{l}\text { Sub-class of ice with } R_{532}>50 \text { representing very high number density ice clouds } \\
\text { typical of mountain-wave-induced PSCs }\end{array}$ \\
\hline & MIPAS PSC Composition Classes \\
\hline Measurement Tech & que: Limb-scanning, Michelson interferometer (infrared emission spectra) \\
\hline & Measurement Volume: $3 \mathrm{~km} \times 30 \mathrm{~km} \times 400 \mathrm{~km}$ \\
\hline $\begin{array}{r}\text { Classification Approacl } \\
\text { comp }\end{array}$ & $\begin{array}{l}\text { Bayesian probabilistic scheme attributes a probability, } P_{\mathrm{j}} \text {, for each of three PSC } \\
\text { sitions (STS, NAT, or ice) to each measured cloud spectrum }\end{array}$ \\
\hline STSmix & $\begin{array}{l}P_{\mathrm{STS}}>50 \% \text { : pure STS or mixtures of STS with large NAT particles }(>2-3 \mu \mathrm{m} \\
\text { radius) that are not detectable spectrally }\end{array}$ \\
\hline NAT & $P_{\mathrm{NAT}}>50 \%:$ Small NAT particles $(<2-3 \mu \mathrm{m}$ radius $)$ present \\
\hline NAT_STS & $\begin{array}{l}\text { Both } P_{\mathrm{NAT}} \text { and } P_{\mathrm{STS}} \text { between } 40-50 \% \text { : Small NAT particles }(<2-3 \mu \mathrm{m} \text { radius }) \text { and } \\
\text { STS present }\end{array}$ \\
\hline Ice & $P_{\text {ice }}>50 \%$ : ice particles present \\
\hline Unknown & $P<40 \%$ for all compositions \\
\hline
\end{tabular}

Figure $4 \mathrm{~g}$ shows altitude profiles of PSC particle VD with a vertical resolution of around $3 \mathrm{~km}$ retrieved from the MIPAS observations (Höpfner et al., 2018). Profiles of minimum/maximum and mean PSC VD are available for each MIPAS limb-scan. The limiting profiles confine the values of VD to within their estimated systematic error, which is about $\pm 40 \%$

This article is protected by copyright. All rights reserved. 
in case of STS or NAT PSCs. In the presence of ice PSCs, i.e. typically VD $>5-10 \mu \mathrm{m}^{3} \mathrm{~cm}^{-3}$, the retrieved values represent only lower limits of VD. MIPAS VD retrievals have been compared to global model simulations by Khosrawi et al. (2018). The example of a VD retrieval in Figure 4g refers to the limb scan in Panel (a) indicated by the black triangle. Panels (e) and (f) show the MIPAS PSC composition categories and derived VD near $21 \mathrm{~km}$ altitude for the same day as the spectra in Panel (a) exhibiting the characteristic NAT spectral feature. The location of the corresponding profile with its classification results is highlighted by a triangle and confirms NAT as a result of the Bayesian classifier. In addition, Panel (e) shows the CALIPSO orbit track (light blue curve) corresponding to the CALIOP data shown in Figure 3, revealing the good correspondence for the large homogeneous ice field in the CALIOP curtain, as well as NAT mixtures, enhanced NAT, and STS PSCs in other segments of the orbit.

\subsubsection{Aura MLS}

MLS, which is aboard the Aura spacecraft launched in July 2004, measures thermal emission at millimeter and sub-millimeter wavelengths from the Earth's limb (Waters et al., 2006). Measurements occur along the forward direction of the Aura spacecraft flight track, with a vertical scan from the surface to $90 \mathrm{~km}$ every $24.7 \mathrm{~s}$. Each orbit consists of 240 scans spaced at $1.5^{\circ}(165 \mathrm{~km})$ along track, with a total of almost 3500 profiles per day and latitudinal coverage from $82^{\circ} \mathrm{S}$ to $82^{\circ} \mathrm{N}$. The Level-1 limb radiance measurements are inverted using 2-D optimal estimation (Livesey et al., 2006) to produce profiles of atmospheric temperature and composition. Of particular relevance for PSC studies are MLS measurements of the primary condensable vapors $\mathrm{HNO}_{3}$ and $\mathrm{H}_{2} \mathrm{O}$. The validation of a previous version of the MLS $\mathrm{H}_{2} \mathrm{O}$ and $\mathrm{HNO}_{3}$ data products and error estimations are discussed in detail by Read et al. (2007), Lambert et al. (2007), and Santee et al. (2007). The current version (v4) of MLS data (Livesey et al., 2017) has single-profile precisions (systematic uncertainties) of 4-15\% (4-7 \%) for $\mathrm{H}_{2} \mathrm{O}$ and 0.6 ppbv (1-2 ppbv) for $\mathrm{HNO}_{3}$. The vertical resolution of $\mathrm{MLS} \mathrm{H}_{2} \mathrm{O}\left(\mathrm{HNO}_{3}\right)$ is 3.1-3.5 (3.5-5.5) $\mathrm{km}$, and the horizontal resolution of MLS $\mathrm{H}_{2} \mathrm{O}\left(\mathrm{HNO}_{3}\right)$ is 180-290 (400-550) km. The MLS FOV spans $3 \mathrm{~km}$ in the cross-track direction.

Along its line of sight, PSCs have no direct radiative effect on MLS measurements of gas-phase constituents. However, Lambert et al. (2012) showed that spatially and temporally correlated MLS $\mathrm{HNO}_{3}$ measurements can be used to infer the presence of PSCs through a decrease in gas-phase $\mathrm{HNO}_{3}$ caused by its sequestration into STS particles or by denitrification via large NAT particles. In the Antarctic case shown in Figure 5, the significant depletions of 4-7 ppbv in $\mathrm{HNO}_{3}$ on 24-25 May 2008 at $46 \mathrm{hPa}$ (Panel a) are clear evidence for $\mathrm{HNO}_{3}$ condensation onto STS or NAT particles. The observation is accompanied by enhancements of about 2 ppbv in $\mathrm{HNO}_{3}$ at $68 \mathrm{hPa}$ (Panel b), i.e. at $2 \mathrm{~km}$ lower altitude. This has been interpreted as renitrification caused by the evaporation of solid NAT particles as they sediment from a colder into a warmer region, leaving behind a denitrified layer with a significant $\mathrm{HNO}_{3}$ reduction of several ppbv (Panel a).

There is a strong synergy for PSC studies between MLS and CALIOP, since the Aura and CALIOP spacecraft flew in formation as part of the afternoon "A-Train" satellite constellation until September 2018. The initial A-Train configuration of CALIPSO and Aura (April 2006 to April 2008) resulted in an across-track orbit offset of $\sim 200 \mathrm{~km}$, with the MLS tangent point leading the CALIOP nadir view by about $7.5 \mathrm{~min}$. From April 2008 to September 2018, Aura and CALIPSO were operated to maintain positioning within tightly constrained control boxes,

This article is protected by copyright. All rights reserved. 
such that the MLS tangent point and the CALIOP nadir point on the ground were synchronized to within 10-20 km spatially and about $30 \mathrm{~s}$ temporally. This synergy is illustrated in Figure 6, where panel (a) shows the same CALIOP PSC orbital curtain from Figure 3, and panels (c, d) show the Aura MLS $\mathrm{H}_{2} \mathrm{O}$ and $\mathrm{HNO}_{3}$ along the same orbit track. The MLS water vapor data can be used to calculate $T_{\text {ice, }}$ which along with ambient temperature interpolated from MERRA-2 reanalysis, allow $T_{\text {MERRA-2 }}-T_{\text {ice }}$ to be mapped onto the CALIOP scene (b). The CALIOP PSC composition curtain shows large contiguous areas of ice and NAT mixtures, but with embedded pixels of other compositions causing a degree of visual speckle. The ice scenes from CALIOP (6a) compare quite favorably with the structure in $T_{\text {MERRA-2 }}-T_{\text {ice }}(6 \mathrm{~b})$. The MERRA-2 temperatures fall to as much as $4 \mathrm{~K}$ below $T_{\text {ice }}$ over a spatial scale of a few thousand kilometers, but on the sub-1000 km scale, the MERRA-2 temperature fields are quite smooth (not shown). Consequently, the structure in the $T_{\text {MERRA-2 }}-T_{\text {ice }}$ field shown in Figure $6 \mathrm{~b}$ results from smallscale changes in $\mathrm{MLS} \mathrm{H}_{2} \mathrm{O}$ (and hence $T_{\text {ice }}$ ). Small scale perturbations, perhaps as induced by gravity waves, are not resolved in the MERRA-2 reanalyses. Large regions of $\mathrm{HNO}_{3}$ depletion are seen where abundances have been reduced to low values by sequestration and denitrification by PSCs compared to pre-existing background values of over $12 \mathrm{ppbv}$. Dehydration of the lower stratosphere caused by large-scale $\mathrm{H}_{2} \mathrm{O}$ sequestration and sedimentation of large ice particles produces regions with $\mathrm{H}_{2} \mathrm{O}$ values up to 2-3 ppmv lower than the 5 ppmv background level.

\subsubsection{Ground-Based Lidar Measurements}

Ground-based lidars have been used to probe PSCs for more than 30 years (Iwasaka, 1985; 1986), and they have spawned many comprehensive analyses on PSC characteristics (e.g., Adriani et al., 1995; Biele et al., 2001; Maturilli et al., 2005). Most ground-based sites utilize elastic backscatter lidars at a wavelength of $532 \mathrm{~nm}$, which are the most effective instruments for discriminating among different PSC compositions (Achtert and Tesche, 2014; Di Liberto et al., 2014). Weather permitting, measurements typically occur once or twice daily, and the data are averaged from 5-60 minutes to increase the SNR. Here, we focus on three ground-based lidar stations with extensive records of PSC observations prior to and during the CALIOP data collection period: McMurdo Station, Antarctica $\left(77.85^{\circ} \mathrm{S}, 166.67^{\circ} \mathrm{E}\right)$, with data from $1990-2010$; Dumont d'Urville, Antarctica $\left(66.67^{\circ} \mathrm{S}, 140.00^{\circ} \mathrm{E}\right)$, with data from 1989-2008; and NyÅlesund, Spitsbergen $\left(78.92^{\circ} \mathrm{N}, 11.93^{\circ} \mathrm{E}\right)$, with data from $1995-2017$. This allows us to assess the consistency between CALIOP and ground-based systems during overlapping measurement periods and also enables the investigation of multi-decadal trends by comparing the contemporary CALIOP record with those ground-based records stretching back to the 1980s and 1990s.

\subsection{Factors Affecting Spaceborne PSC Data Intercomparison}

\subsubsection{STS and NAT Particle Detection Sensitivities}

Lidar backscatter (CALIOP), infrared emission/scattering (MIPAS), and microwave emission (MLS) are fundamentally different physical techniques for probing air parcels containing PSCs. In this section, we will examine the theoretical sensitivity of each technique to the presence of STS and NAT PSC particles. Figure 7 (bottom panel) shows the equilibrium condensed $\mathrm{HNO}_{3}$ fraction in STS (Carlsaw et al., 1995) as a function of temperature relative to the frost point ( $\left.T-T_{\text {ice }}\right)$ and relative to the NAT equilibrium temperature ( $T$ - $T_{\mathrm{NAT}}$; Hansen and Mauersberger, 1988) for $0.1 \mathrm{ppbv}_{2} \mathrm{SO}_{4}, 5 \mathrm{ppmv} \mathrm{H}_{2} \mathrm{O}$, and $12 \mathrm{ppbv}$ total $\mathrm{HNO}_{3}$ at a pressure of

This article is protected by copyright. All rights reserved. 
$46 \mathrm{hPa}$. Figure 7 (top panel) shows STS $R_{532}$ (purple curve) and STS VD (red curve) as a function of condensed $\mathrm{HNO}_{3}$ fraction in STS for these same conditions. The CALIOP threshold for detecting STS in a single 180 -m vertical $\times 135-\mathrm{km}$ horizontal measurement pixel is $R_{532} \approx$ 1.25 , which is equivalent to $7 \%$ condensed $\mathrm{HNO}_{3}$ fraction in STS. The MIPAS threshold for detecting the presence of particles within its limb path is $\mathrm{VD}=0.2-0.3 \mu \mathrm{m}^{3} \mathrm{~cm}^{-3}$, which is equivalent to $4.7-7.9 \%$ condensed $\mathrm{HNO}_{3}$. By comparison, the detection limit (measurement uncertainty) of MLS gas-phase $\mathrm{HNO}_{3}(0.6 \mathrm{ppbv})$ is equivalent to $5 \%$ condensed $\mathrm{HNO}_{3}$ fraction in STS within its limb path. Therefore, both MIPAS and MLS can sense the presence of STS within their limb paths somewhat better than CALIOP can detect STS on a pixel-by-pixel basis. It should be noted that under non-volcanic conditions, the STS detection limit for ground-based lidars can be as low as $R_{532} \approx 1.1$ because of a higher SNR in comparison to CALIOP (Achtert et al., 2012), suggesting that ground-based lidars may be more sensitive to the presence of STS than any of the contemporary spaceborne instruments.

Figure 8 compares the detection sensitivity of CALIOP, MIPAS, and MLS for a variety of STS/NAT mixtures corresponding to a range of NAT number densities and NAT effective

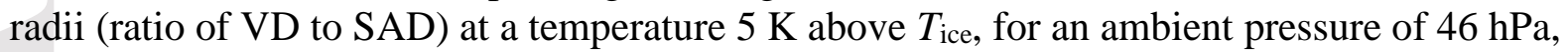
0.1 ppbv $\mathrm{H}_{2} \mathrm{SO}_{4}, 5$ ppmv $\mathrm{H}_{2} \mathrm{O}$, and 12 ppbv total $\mathrm{HNO}_{3}$. The NAT particles are modeled as spheroids with $\varepsilon=0.9$ using T-matrix calculations assuming a power-law NAT size distribution; otherwise, the STS/NAT mixtures are modeled as in Pitts et al. (2009) and Lambert et al. (2012). The green solid line represents the uptake in NAT particles of $0.6 \mathrm{ppbv} \mathrm{HNO}_{3}$ based on the MLS detection threshold within the MLS limb path. The dashed red lines represent condensed $\mathrm{HNO}_{3}$ in NAT particles equivalent to VD $=0.2$ (lower line) and 0.3 (upper line) $\mu \mathrm{m}^{-3} \mathrm{~cm}^{-3}$, based on the MIPAS limits for detecting the presence of particles within its limb path. The dashed curves represent the CALIOP PSC detection thresholds for a single $180-\mathrm{m}$ vertical $\times 135$-km horizontal measurement pixel in terms of $R_{532} \approx 1.25$ (purple) and $\beta_{\perp} \approx 2.5 \times 10^{-6} \mathrm{~km}^{-1} \mathrm{sr}^{-1}$ (blue). The area below any single curve or line represents NAT number density/effective radius combinations that are below the detection limit for that particular measurement technique, and the gray shaded area is below the detection limits of all three satellite instruments. The figure shows that for CALIOP, $\beta_{\perp}$ is more sensitive than $R_{532}$ to the presence of NAT particles over almost the entire range of NAT number densities and effective radii, which is why $\beta_{\perp}$ is used instead of $R_{532}$ for detection of CALIOP NAT mixture (and ice) PSCs. The figure also indicates that both MIPAS and MLS are more sensitive than CALIOP to the presence of large (radii $\gtrsim 4-5 \mu \mathrm{m}$ ), low number density ( $\lesssim$ $5-8 \times 10^{-4} \mathrm{~cm}^{-3}$ ) NAT particles ("NAT rocks"), whereas CALIOP $\beta_{\perp}$ appears to be more sensitive than MIPAS or MLS to synoptic-scale NAT mixtures and enhanced NAT mixtures. Note that while MIPAS is sensitive to the presence of NAT rocks in terms of equivalent VD within its limb FOV, such large NAT particles cannot be distinguished spectrally using the NI. It should also be noted that ground-based lidars may be superior to the satellite instruments in terms of sensitivity to NAT mixtures because of their higher SNR. Thus ground-based lidars are suited for

This article is protected by copyright. All rights reserved. 
detailed process-oriented studies (e.g. Biele et al., 2001) but cannot provide the large-scale coverage that spaceborne instruments offer.

The sensitivity differences between CALIOP, MLS, and MIPAS are reflected in the fact that the onset of the Antarctic PSC season is often detected slightly later by CALIOP ( 1-7 days) than by MLS (Lambert et al., 2016) and MIPAS (Spang et al., 2016; 2018).

\subsubsection{Differences in Spatial/Temporal Sampling}

The downward-viewing CALIOP and the limb-viewing MIPAS and MLS provide vastly different perspectives of PSC scenes. In this section, we discuss the differences in spatial sampling of PSCs by these instruments and how these differences impact quantitative comparisons of the datasets.

A distinct advantage of CALIOP is its very high spatial resolution with PSC products assembled on a 5-km along-track $\times 180$-m vertical grid along each of the 14-15 orbits per day. However, CALIOP PSCs are not always detected at the 5-km scale; more tenuous PSCs are detected at the $15-\mathrm{km}, 45-\mathrm{km}$, or $135-\mathrm{km}$ scales through successive averaging. The MIPAS and MLS limb-viewing instruments have much larger measurement volumes: $\sim 3 \mathrm{~km}$ vertical $\times \sim 30$ $\mathrm{km}$ across track $\times \sim 400 \mathrm{~km}$ horizontal for MIPAS; and 3.1-3.5 (3.5-5.5) km vertical $\times 3 \mathrm{~km}$ cross-track $\times 180-290(400-550) \mathrm{km}$ horizontal for MLS $\mathrm{H}_{2} \mathrm{O}\left(\mathrm{HNO}_{3}\right)$, both comprising hundreds of CALIOP measurement samples. The CALIOP data curtains (e.g., Figure 3) typically show that there is considerable inhomogeneity along an orbit in both PSC coverage (cloudy vs. clear pixels) and inferred PSC composition. The limb-viewing instruments are insensitive to this inhomogeneity, and as a result, MIPAS PSC products and MLS gas species abundances are averages over their respective limb measurement volumes.

There tends to be excellent agreement in PSC compositon between CALIOP and MIPAS for CALIOP scenes in which a single PSC composition is predominant, such as the Antarctic PSC curtain from 24 August 2011 shown in Figure 9. The black curves in the figure trace notional MIPAS limb-viewing paths horizontally across the scene at 2-km tangent altitude intervals, assuming the MIPAS FOV to be aligned with the CALIPSO orbit. Note that the other dimensions of the MIPAS sampling volume are $\sim 3 \mathrm{~km}$ in the vertical and $30 \mathrm{~km}$ across-track (into the plane of the figure). The MIPAS composition profile indicated by the symbols in the figure is the closest match in space and time for this CALIOP curtain, occurring within $56 \mathrm{~km}$ spatially and within 1.6 hours in time. In this spatially homogeneous example, CALIOP shows that ice is the predominant composition of the PSC, which is borne out by the MIPAS retrieval indicating ice at altitudes from $18-23 \mathrm{~km}$.

The agreement between CALIOP and MIPAS is less satisfactory for spatially inhomogeneous scenes such as the CALIOP Antarctic PSC orbital curtain from 29 June 2011 shown in Figure 10. This figure again shows notional MIPAS limb-viewing paths across the scene at 2-km tangent altitude intervals, assuming the MIPAS FOV to be aligned with the CALIPSO orbit. The MIPAS composition profile shown in Figure 10 is the closest spatial and temporal match for this CALIOP curtain, occurring within $64 \mathrm{~km}$ spatially and nearly perfectly matched in time. The MIPAS retrieval indicates ice PSCs at limb path tangent point altitudes from about 16-21 km and a NAT PSC at $23 \mathrm{~km}$, whereas CALIOP shows mostly STS and NAT mixtures along the MIPAS limb paths. Although ice is sparse in the CALIOP scene, the MIPAS

This article is protected by copyright. All rights reserved. 
retrieval suggests that the ice has a disproportionate influence on the spectrum measured over the limb path.

\subsubsection{Intercomparisons of PSC Datasets}

\subsubsection{CALIOP vs MIPAS}

The seasonal evolution of PSC areal coverage from CALIOP and MIPAS were compared for select years in Spang et al. (2018) and Tritscher et al. (2019). Figure 11 shows the seasonal evolution of PSC areas over the Antarctic in 2009 as observed by CALIOP (left column) and MIPAS (right column). Shown are total PSC areal coverage (top row), as well as areas for three corresponding CALIOP/MIPAS composition classes. Overall, the seasonal evolution of the PSC areal coverages is qualitatively similar. However, MIPAS exhibits larger maximum values in total areal coverage than CALIOP (Figure 11, top panels), likely due to (1) MIPAS's larger sampling volume, which does not account for partly cloudy conditions frequently observed by CALIOP as discussed earlier in Section 2.2.2, and (2) MIPAS's higher sensitivity to the presence of large $(\approx 4-5 \mu \mathrm{m})$, low number density $\left(\lesssim 5-8 \times 10^{-4} \mathrm{~cm}^{-3}\right)$ NAT particles. In addition, MIPAS cloud detection sensitivity is biased towards the cloud top which causes difficulty in retrieving accurate cloud bottom information. To partially compensate for this effect, the MIPAS cloud presence and composition statistics shown here assume a nominal cloud thickness of up to $4 \mathrm{~km}$ below cloud top for cloud-affected spectra; however, this simplification still appears to underestimate cloud presence at lower altitudes during much of the season.

For a more quantitative assessment of the consistency between CALIOP and MIPAS in terms of overall PSC coverage, a correlation analysis was performed on daily CALIOP and MIPAS PSC spatial volumes (PSC areas integrated over altitudes from 16-30 km) for the 20062011 period when both instruments were making measurements. The MIPAS volumes are based on the VD dataset (Höpfner et al., 2018) rather than the Bayesian composition classification results of Spang et al. (2016). The VD retrieval more accurately identifies cloud top, but still suffers from a lack of definition of cloud bottom for optically thick PSCs. This may produce artefacts that lead to overestimation of PSC thickness. We found that MIPAS PSC volumes were on average larger than the CALIOP PSC volumes by factors of 1.38 in the Antarctic and 1.63 in the Arctic. Factors contributing to the differences between the instruments include uncertainty in MIPAS cloud bottom as well as underestimation of PSC coverage by CALIOP due to its conservative PSC detection thresholds. However, a more important factor is that the differences are due to overestimation of PSC coverage by MIPAS since there is no accounting for cloud patchiness within its large limb-viewing sampling volume, whereas CALIOP determines cloudiness on a pixel-by-pixel basis. One would then conclude from the results that PSCs are typically patchier in the Arctic (larger scaling factor) than in the Antarctic, which appears to be true from examination of the orbital scenes, but this needs to be confirmed by more detailed studies of the CALIOP data in the future. Figure 12 shows the combined CALIOP and scaled MIPAS time series of daily PSC spatial volume for the years 2002-2018, capturing the regular "heartbeat" of Antarctic seasonal PSC occurrence (panel a) and the much more irregular "heartbeat" of Arctic PSC seasonal occurrence (panel b). This is the first climatological record of measurement-based PSC spatial volume spanning 16 years for both polar regions, which can be

This article is protected by copyright. All rights reserved. 
used for chemistry-climate model validation and improvements for a better prediction of future polar ozone loss in a changing climate.

A valuable aspect of intercomparing CALIOP and MIPAS PSC observations is that the spectral identification of PSC composition by MIPAS provides crucial underpinning of the PSC composition that is inferred indirectly from the CALIOP optical measurements. Generally favorable comparisons between MIPAS and earlier (Version 1) CALIOP PSC composition results were found by Höpfner et al. (2009) using the 2-CR method (Section 2.1.1.2) and more recently by Spang et al. (2016) using a new Bayesian classifier approach. Most recently, Höpfner et al. (2018) found that for coincident PSC scenes classified as predominantly (> 50\%) STS by CALIOP, there was good agreement between the magnitudes and vertical profile shapes of CALIOP and MIPAS particle VD. Here, we extend the results of Höpfner et al. (2009) by comparing the CALIOP v2 and MIPAS Bayesian PSC composition classifications for the 20062011 overlap period using more stringent coincidence criteria $(<1$ hour in time and $<100 \mathrm{~km}$ in radial distance from the MIPAS tangent point) and further restricting the comparison to homogeneous CALIOP scenes, defined by the criteria that more than $50 \%$ of the CALIOP individual 5-km measurement samples within the MIPAS FOV are PSCs and more than $75 \%$ of those samples are classified as a single CALIOP composition. Due to the stringent coincidence criteria, no suitable homogeneous scenes were found for wave ice or enhanced NAT mixtures. In other words, these PSC sub-classes typically occur on smaller spatial scales than encompassed by the MIPAS measurement volume. The results for the Antarctic are shown in Figure 13a and can be summarized as follows (note that labels have been excluded for matches $<1 \%$ ):

- For CALIOP scenes that are predominantly ice, there is excellent (> 99\%) agreement with MIPAS ice.

- For CALIOP scenes that are predominantly STS, about $77 \%$ of the MIPAS classifications are either STSmix (39\%) or NAT_STS (38\%). MIPAS classifies about $14 \%$ of the CALIOP STS scenes as ice, likely because ice can be the dominant composition in a spectral sense within the MIPAS FOV even though STS occupies most of the FOV from a spatial point of view.

- For CALIOP scenes that are predominantly NAT mixtures, there is good $(73 \%)$ correspondence with MIPAS NAT.

- The MIPAS NAT_STS class is a better match to the CALIOP STS class than to the CALIOP NAT mixture class. A possible conclusion is that the MIPAS NAT_STS class has only a limited additional information content compared to the STSmix class.

Since PSCs occur much less frequently over the Arctic than over the Antarctic (Pitts et al., 2018; Spang et al., 2018), there are many fewer suitable MIPAS-CALIOP coincidences in

This article is protected by copyright. All rights reserved. 
the Arctic, yielding comparison results with a reduced statistical significance. Nevertheless, we can summarize the results for the Arctic (Figure 13b) as follows:

- For CALIOP scenes that are predominantly ice, there is a $100 \%$ match with MIPAS ice. However, note that there are only 3 Arctic ice PSC cases that met the stringent requirements for both coincidence and scene homegeneity.

- For CALIOP scenes that are predominantly STS, about $85 \%$ of the MIPAS classifications are either STSmix (56\%) or NAT_STS (29\%).

- For CALIOP scenes that are predominantly NAT mixtures, only $18 \%$ are classified as NAT by MIPAS, with the remaining $82 \%$ classified as STSmix. This is a striking contrast to the Antarctic, where agreement of MIPAS NAT with CALIOP NAT mixture scenes was $73 \%$. One possible explanation is that, on average, the size of NAT particles may be larger in the Arctic than in the Antarctic and, thus, less likely to fall into the MIPAS NAT class, which is limited to NAT particles with radii $<2-3 \mu \mathrm{m}$. Another contributing factor may be that the generally more tenuous and spatially patchy PSCs in the Arctic are more difficult to detect by MIPAS limb sounder with its large field-of-view. We have no conclusive evidence that either of these is the primary cause, so the discrepancy remains unresolved.

\subsubsection{CALIOP vs. Aura MLS}

The near-simultaneous and collocated measurements of gas phase $\mathrm{HNO}_{3}$ and $\mathrm{H}_{2} \mathrm{O}$ by Aura MLS have provided additional validation of the PSC composition inferred from CALIOP data and valuable new insight into PSC formation and growth kinetics. The basic approach was introduced by Lambert et al. (2012) and involves comparing the observed temperature-dependent uptake of $\mathrm{HNO}_{3}$ by CALIOP-detected PSCs of different compositions with modeled uptake of $\mathrm{HNO}_{3}$ for equilibrium STS and NAT. The technique was refined by Pitts et al. (2013), who restricted the CALIOP-MLS comparisons to homogeneous scenes: those with > 75\% PSC coverage over the effective MLS measurement volume, with 2/3 of those PSCs identified as a single CALIOP PSC composition. Analysis of CALIOP and MLS observations from 2006-2018 (see Figure 14) show that both STS and ice PSCs are near thermodynamic equilibrium with the gas phase, as expected from theoretical considerations. Lambert and Santee (2018) used these PSC thermodynamic equilibrium temperatures as independent absolute references to evaluate the overall accuracy and precision of polar lower stratospheric temperatures reported in several contemporary reanalysis datasets. Figure 14 also shows that the temperature dependence of NAT mixture PSCs is bimodal in nature, with one mode near $T_{\mathrm{NAT}}$ and a second mode near $T_{\mathrm{STS}}$. Examining temperature histories along back trajectories from CALIOP observation points for the 2009-10 Arctic winter, Pitts et al. (2013) showed this bimodality is likely a consequence of different exposure times of air parcels to temperatures below $T_{\mathrm{NAT}}$. The mode near the STS equilibrium temperature represents air parcels with relatively brief exposure to temperatures below $T_{\mathrm{NAT}}$, in which the uptake of $\mathrm{HNO}_{3}$ is dominated by the much more numerous STS droplets at the lower temperatures (Biele et al., 2001). The NAT mixture mode near NAT equilibrium corresponds to parcels exposed to temperatures below $T_{\mathrm{NAT}}$ for extended periods of time, allowing a larger fraction of the gas-phase $\mathrm{HNO}_{3}$ to condense onto the thermodynamically favored NAT particles and bringing the mixture closer to NAT equilibrium. The bimodality seen

This article is protected by copyright. All rights reserved. 
in the NAT mixture histograms for the entire CALIOP data set is likely related to parcel temperature histories, but this cannot be confirmed without further analyses.

\subsubsection{CALIOP vs. Ground-Based Lidar}

Even though both CALIOP and ground-based lidars employ similar measurement approaches, comparison of PSC observations between them is not straightforward, as there are important operational differences between the two. A major limitation of ground-based lidars is that the quality and quantity of the data is strongly impacted by the presence of tropospheric clouds, which render measurements in the stratosphere above these clouds impossible when the optical depths are sufficient to fully attenuate the lidar's signal. This may induce a bias in PSC statistics, since the formation of PSCs is often associated with tropospheric meteorology, in particular deep tropospheric cloud systems (Wang et al., 2008; Adhikari et al., 2010; Achtert et al., 2012). Spatial inhomogeneity is another confounding factor in comparing CALIOP and ground-based lidar PSC data. Each CALIOP 5-km PSC measurement sample is a "snapshot" of the atmosphere taken in less than $1 \mathrm{~s}$, whereas the ground-based lidar PSC products are generally based on 5-to-60 minute average profiles obtained once or twice daily.

An unambiguous point-to-point comparison of coincident ground-based lidar and CALIOP PSC observations is practically impossible, but statistical comparison of a large number of observations can overcome some of these issues and has been pursued by several groups. One such example is the comparison by Córdoba-Jabonero et al. (2013) of volume depolarization ratio - an indicator of PSC particle shape - measured by the ground-based lidar at Belgrano II $\left(77.87^{\circ} \mathrm{S}, 34.62^{\circ} \mathrm{W}\right)$ with CALIOP data for three Antarctic winters (2009-2011). They found a relatively good agreement, with a weak dependence on the distance between the CALIOP measurement footprint and the ground-based lidar. This suggests relatively homogeneous PSC scenes, likely because the Belgrano II station lies well inside the Antarctic polar vortex.

Snels et al. (2019) performed a statistical comparison of PSCs classified by composition derived from ground-based lidar data at McMurdo Station, Antarctica and from nearby CALIPSO overpasses. Figure 15 compares PSC occurrence and inferred composition from CALIOP (top panel) and the McMurdo ground-based lidar (bottom panel) as a function of day and altitude during the 2006 Antarctic winter. There is largely good agreement between the datasets in the general features of the PSC season, in terms of the occurrence of each composition class and its altitude dependence during the season. One can observe in both panels the predominance of NAT mixtures and the appearance of ice (blue) in the second half of July. However, there are many differences in detail with regard to the daily observations, confirming that the quality of point-to-point comparisons of CALIOP and the ground-based station is limited owing to the high geographic variability of PSCs. A compromise must be reached between using a statistically small sample of near-perfect matches versus using a larger, statistically more significant sample including greater match distances.The analysis of Snels et al. (2019) accepted encounters coincident with CALIPSO overpass times and at match distances $\leq 100 \mathrm{~km}$, over which PSCs can differ considerably even in the Antarctic (see Figure 10). A similar comparison has been performed more recently on a larger dataset of quasi-coincident PSC observations at Concordia (Dome C, Antarctica) by ground-based lidar and CALIOP (Snels et al., 2021). In this

This article is protected by copyright. All rights reserved. 
region with no significant orographic features, large contiguous PSCs were observed, and very good agreement was obtained at match distances $\leq 100 \mathrm{~km}$.

On the other hand, the statistical comparison of many coincident observations shows an acceptable agreement, considering the different observation geometries and other possible biases. Such a statistical comparison was presented by Pitts et al. (2018) as 2D (month vs. altitude) histograms of CALIOP v2 PSC sighting frequency near McMurdo for 2006-2010 and 2006-2017 and near Ny-Ålesund for 2006-2017, noting the qualitative similarity to analogous histograms of ground-based data published by Di Liberto et al. (2014) and Massoli et al. (2006). We reexamine the 2006-2010 period of overlapping CALIOP and McMurdo observations by assuming a PSC was present in the McMurdo ground-based data when $R_{532}>1.25$ in at least three consecutive $150-\mathrm{m}$ bins in the vertical profile between $12-26 \mathrm{~km}$. For comparison, the CALIOP v2 PSC data was confined to measurements lying with $\pm 1^{\circ}$ latitude and $\pm 3.5^{\circ}$ longitude of McMurdo. Both data sets were accumulated in 1 month $\times 2 \mathrm{~km}$ vertical bins between June and September, and average sighting frequencies (number of days with PSCs divided by the total number of observation days) over the 5-year period were calculated for each bin. Figure 16 shows the resulting histograms for the ground-based lidar (left) and CALIOP (right). A bin-by-bin correlation analysis between the two histograms shows a Pearson correlation coefficient of $\sim 0.93$, and the ground-based sighting frequencies are only $\sim 7 \%$ lower than the CALIOP sighting frequencies on average. The conclusion is that the ground-based and CALIOP PSC frequency datasets for McMurdo are consistent, with relatively minor quantitative difference due to different sampling geometries.

\subsection{PSC Characteristics: Seasonal, Geographical and by Composition}

The high spatial resolution CALIOP measurements, underpinned by the MIPAS spectral information on composition and $\mathrm{MLS} \mathrm{HNO}_{3}$ and $\mathrm{H}_{2} \mathrm{O}$ measurements, have provided a wealth of new information on the detailed spatial patterns of PSC occurrence and composition on vortexwide scales. In this section, we describe the seasonal evolution and interannual variability of Antarctic and Arctic PSC spatial coverage and show how PSC composition varies seasonally in a climatological sense. We present climatological mean PSC occurrence polar maps and relate zonal mean distributions of PSC occurrence to distributions of temperature and to the PSC precursor gases $\mathrm{HNO}_{3}$ and $\mathrm{H}_{2} \mathrm{O}$. Finally, we investigate the possibility of temporal trends in PSC occurrence by comparing CALIOP data with ground-based lidar and historical (1978-1989) SAM II solar occultation data.

\subsubsection{Overall Seasonal Evolution}

The vortex-wide, seasonal evolution of PSC occurrence is depicted by the total areal coverage of PSCs over the polar region as a function of altitude and time (Pitts et al., 2018; Spang et al., 2018). Temperatures cold enough for PSC formation/existence are typically confined to the polar vortex, which in the Antarctic is large, relatively axisymmetric, and generally similar from year to year. Hence, it is not surprising that the seasonal evolution of PSC coverage in the Antarctic has relatively little interannual variability. The climatological mean of Antarctic PSC areal coverage observed by CALIOP (2006-2018) is depicted in Figure 17a. The onset of the Antarctic PSC season generally occurs in mid-May with the specific date dependent on the details of the evolving Antarctic polar vortex such as its coldness and shape, which can vary significantly from year to year. The spatial extent of Antarctic PSCs typically peaks in July

This article is protected by copyright. All rights reserved. 
and August when the vortex is largest and coldest and then diminishes markedly in September and approaches zero as temperatures warm in mid to late October. PSCs extend in altitude from near the tropopause up to $>25 \mathrm{~km}$, but the altitude of maximum areal coverage systematically decreases from above $20 \mathrm{~km}$ early in the season to near $15 \mathrm{~km}$ by September, in conjunction with a downward shift in the axis of coldest temperatures as the vortex warms from above.

In contrast, in the Northern Hemisphere the more irregular underlying surface topography leads to stronger upward-propagating planetary wave activity, which typically leads to a weaker and more distorted Arctic polar vortex than its Antarctic counterpart. As a result, temperatures in the Arctic polar vortex are generally higher than in the Antarctic and highly variable from yearto-year. Sudden stratospheric warmings can severely disrupt or even completely break down the vortex in mid-winter, resulting in a lack of PSCs at these times due to the warm stratospheric temperatures. The climatological mean seasonal evolution of Arctic PSC areal coverage as observed by CALIOP is vastly different from the mean Antarctic picture, see Figure 17b. Pitts et al. (2018) showed that the climatological Arctic mean PSC coverage is unlike any single year in the 13-year CALIOP Arctic data record; hence the climatological mean is not a very useful representation of the seasonal behavior of Arctic PSCs in a model. Rather, orographic/upper tropospheric forcing and the dynamic variability of the Arctic vortex can produce significant deviations from the climatological mean PSC coverage depicted in Figure 17b.

Pitts et al. (2018) quantified the interannual variability in PSC coverage by examining the range of daily values of PSC spatial volume (daily area coverage integrated over altitude) over the 2006-2017 period. Figure 18a shows the updated time series of the 13-year (2006-2018) mean, standard deviation, and range of daily values of Antarctic PSC spatial volume, with maximum and minimum values color-coded according to the year in which they occurred. At the peak of the season in July, the relative standard deviation in PSC spatial volume is about $\pm 25 \%$, a value similar to the interannual variability in maximum PSC area observed by MIPAS between 2002 and 2011 (Spang et al., 2018). The year-to-year variability in Arctic PSC coverage is shown in Figure 18b, which depicts the time series of the CALIOP 12-year mean daily PSC spatial volumes over the Arctic along with the standard deviations, maxima, and minima. The relative year-to-year variability in the PSC spatial volume in the Arctic is much larger than in the Antarctic, with the relative standard deviations exceeding $100 \%$ for most days. The vast difference in PSC spatial coverage between the hemispheres is also evident in Figure 18, with mid-winter Antarctic spatial volumes 6-8 times larger on average.

\subsubsection{PSC Coverage by Composition Class}

The high spatial resolution CALIOP data record is also providing the first detailed depiction of the seasonal evolution of PSCs by composition (Pitts et al., 2018). Figure 19 shows the 2006-2018 climatological mean relative Antarctic PSC spatial coverage (compositionspecific area normalized by total area) observed by CALIOP for (a) STS; (b) NAT mixtures, including enhanced NAT mixtures; and (c) ice, including wave ice. Figure 19d shows the 13year mean contour plot of $T-T_{\mathrm{NAT}}$, where $T$ is the ambient temperature from MERRA-2 reanalyses, and $T_{\mathrm{NAT}}$ (Hanson and Mauersberger, 1988) is calculated using Aura MLS gas-phase $\mathrm{HNO}_{3}$ and $\mathrm{H}_{2} \mathrm{O}$ partial pressures in cloud-free regions. To avoid the highly variable onset period in constructing a representative climatology, the analysis is restricted to days and altitudes where PSCs were observed in at least 6 of the 13 Antarctic seasons covered by CALIOP, as demarcated by the thick black contour line on each of the color panels in Figure 19. For STS and NAT

This article is protected by copyright. All rights reserved. 
mixtures, PSC onset occurred in at least 6 years by around 20 May, but as mentioned in Section 2.2.1, MLS and MIPAS detected PSCs earlier in some years (by up to one week).

Lambert et al. (2016) showed that the composition assigned to early season Antarctic PSCs observed by CALIOP depends on the cooling rate along an air parcel trajectory, which is determined by both the shape of the vortex and relative position of the cold pool. In slow cooling situations, much of the available $\mathrm{HNO}_{3}$ is sequestered into NAT particles that form at temperatures between $T_{\mathrm{NAT}}$ and $T_{\mathrm{STS}}$, and the growth of STS droplets at lower temperatures is limited. These PSCs are an external mixture of SSA and low number density, large NAT particles that are classified as CALIOP NAT mixtures if they are detectable through enhanced $\beta_{\perp}$. Depending on the NAT particle number density and size, such PSCs may fall below the CALIOP $\beta_{\perp}$ detection threshold and would not be detectable by CALIOP, but are detectable by MLS through the uptake of $\mathrm{HNO}_{3}$. In situations with rapid cooling, little $\mathrm{HNO}_{3}$ is condensed onto the NAT particles before the temperature drops below $T_{\mathrm{STS}}$, when STS droplets start to grow rapidly via $\mathrm{HNO}_{3}$ and $\mathrm{H}_{2} \mathrm{O}$ condensation. Such PSCs would likely be classified by CALIOP as STS, even though the clouds actually contain low number density (undetectable) NAT particles. A similar dependence of assigned CALIOP PSC composition on vortex shape and cooling rate was found during the 2009-2010 Arctic winter (Pitts et al., 2011, Pitts et al., 2013).

The onset of ice PSCs (Figure 19c) typically occurs around mid-June when temperatures

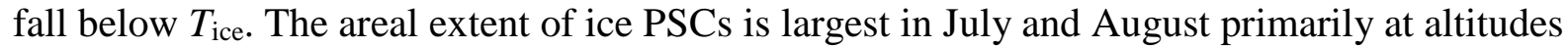
below $20 \mathrm{~km}$, but ice is rarely the predominant composition over vortex-wide scales. However, analyses of MIPAS data by Spang et al. (2018) indicate that ice may at times be the predominant composition between $82^{\circ} \mathrm{S}$ and the South Pole. STS is the most prevalent composition above 20 $\mathrm{km}$ until mid-June and then again at lower altitudes in September and October. The early-season predominance of STS above $20 \mathrm{~km}$ corresponds to the region of largest temperature departures below $T_{\mathrm{NAT}}$, which is consistent with an enhanced liquid particle growth regime. The predominance of STS late in the season at altitudes below $20 \mathrm{~km}$ may be an indication that efficient NAT nuclei have been removed through sedimentation of PSC particles during the winter. Above $\sim 17 \mathrm{~km}$ during July through mid-September, NAT mixtures are the predominant composition which may be an indication of a continuing resupply of nuclei in descending air masses (see Section 3). NAT mixtures is also by far the predominant composition below $17 \mathrm{~km}$ in May and early June which corresponds to a region of temperatures near or just below $T_{\mathrm{NAT}}$, where STS particle growth is thermodynamically impossible.

Antarctic PSC composition is summarized by Figure 20a, which shows the mean seasonlong vertical profile of relative PSC spatial coverage (composition-specific area normalized by total PSC area). NAT mixtures is the majority season-long PSC composition above $18 \mathrm{~km}$, which is consistent with Figure 19b. The relative maxima in season-long STS fraction near $25 \mathrm{~km}$ and $15 \mathrm{~km}$ reflect the early season and late season maxima in STS coverage shown in Figure 19a. The season-long ice maximum near $12 \mathrm{~km}$ may be produced by upper tropospheric cirrus.

Because of extremely high interannual variability, the only meaningful climatological picture of Arctic PSC composition is the analogous mean season-long vertical profile of relative PSC spatial coverage shown in Figure 20b. STS and NAT mixtures are the prevalent Arctic PSC compositions as expected. The two occur in roughly equal proportions above $24 \mathrm{~km}$, while NAT

This article is protected by copyright. All rights reserved. 
mixtures is the predominant composition below $24 \mathrm{~km}$. As in the Antarctic, upper tropospheric cirrus produces the season-long ice maximum near $12 \mathrm{~km}$ in the Arctic.

\subsubsection{Geographical and Zonal Mean Distribution}

Overall PSC occurrence is not typically zonally symmetric, but instead exhibits distinct longitudinal patterns. Figure 21 (top row) shows monthly mean polar maps of overall Antarctic PSC frequency observed by CALIOP at $\theta=500 \mathrm{~K}(\sim 20 \mathrm{~km}$ altitude) for 2006-2018. For context, the mean location of the edge of the vortex (solid black line) and the boundaries of the regions with mean $T<T_{\mathrm{NAT}}$ (solid red line) and $T<T_{\text {ice }}$ (solid white line) are overlaid on the CALIOP maps. PSC occurrence is roughly bounded by the $T<T_{\mathrm{NAT}}$ contour and increases poleward with the highest occurrence frequencies ( $>60 \%$ ) generally located within the region of $T<T_{\text {ice. The }}$ contours of PSC occurrence frequency and the cold pool are not zonally symmetric, but instead are pushed slightly off the pole towards the Greenwich Meridian (GM). This zonal asymmetry is especially pronounced in July-September with the maximum PSC occurrence frequency at $0^{\circ}$ $90^{\circ} \mathrm{W}$ longitude near the base of t'ie Antarctic Peninsula. As discussed later in Section 4.2, the enhancement in PSC occurrence at longitudes near the Antarctic Peninsula is due to frequent mountain wave activity in this region and the large-scale upper tropospheric forcing events, which are more frequent at these longitudes. A local maximum in PSC occurrence near the Antarctic Peninsula was also observed by MIPAS (Spang et al., 2018).

The high resolution of CALIOP data captures the detailed spatial patterns in PSC occurrence by composition. Figure 21 shows the climatological mean geographical distributions of each composition class, illustrating the preferred occurrence patterns of STS (second row), NAT mixtures (third row), and ice PSCs (bottom row) at $\theta=500 \mathrm{~K}$. STS PSCs are widespread during June at this level, but more limited afterwards with occurrence frequencies generally less than $10 \%$ in the interior of the vortex during July-August and less than 5\% during September. NAT mixtures, on the other hand, are relatively widespread over much of the vortex, especially during July and August when occurrence frequencies exceed 35\%. The ring of higher NAT mixture occurrence in July over East Antarctica between $70^{\circ}-75^{\circ} \mathrm{S}$ is consistent with the NAT belt described in detail in the case study of Section 4.2. Ice PSC occurrence is aligned reasonably well with the region of mean $T<T_{\text {ice }}$ over the interior of the vortex generally poleward of $70^{\circ} \mathrm{S}$, with a distinct maximum in July and August near the base of the Antarctic Peninsula arising from the frequent mountain wave and upper-tropospheric forcing events in this region.

Despite the high interannual variability in overall PSC areal coverage in the Arctic, the geographical pattern of overall PSC occurrence there is quite regular climatologically, as illustrated in monthly mean PSC occurrence frequency maps for December-February shown in Figure 22 for CALIOP (2006-2018 average). Arctic PSCs are primarily confined to longitudes from about $60^{\circ} \mathrm{W}$ to $120^{\circ} \mathrm{E}$, a region that corresponds to the climatologically favored location of the Arctic vortex (e.g. Zhang et al., 2016), which is influenced by enhanced zonal wavenumber 1 activity, pushing the vortex off the North Pole towards Eurasia. A similar spatial pattern was noted in MIPAS PSC occurrence in the Arctic (Spang et al., 2018). Due to the extreme interannual variability in Arctic PSC composition, long-term average composition-specific maps are not meaningful and are not presented here.

The interplay between temperature and condensable vapor $\left(\mathrm{HNO}_{3}\right.$ and $\left.\mathrm{H}_{2} \mathrm{O}\right)$ supplies in PSC occurrence is illustrated well in Antarctic monthly mean (May through September) crosssections displayed using the quasi-Lagrangian equivalent latitude (EqLat) vs. potential

This article is protected by copyright. All rights reserved. 
temperature $(\theta)$ coordinate system. Figure 23 shows the cross-sections of CALIOP 2006-2018 monthly average Antarctic PSC occurrence frequency, cloud-free $\mathrm{MLS} \mathrm{HNO}_{3}$ and $\mathrm{H}_{2} \mathrm{O}$, MERRA-2 $T$, and $T-T_{\mathrm{NAT}}$ in respective rows. Note the sharp gradients in $\mathrm{HNO}_{3}$ and $\mathrm{H}_{2} \mathrm{O}$ between the interior and "collar" regions of the vortex that are clearly captured in the EqLat/ $\theta$ cross-sections. The interior of the vortex at EqLat $<-75^{\circ}$ and $\theta=400-500 \mathrm{~K}$ is still largely unperturbed in May but becomes severely denitrified and dehydrated by July. Although temperatures are low near the vortex center in July and August, there are relative minima in PSC occurrence near $\theta=450 \mathrm{~K}$, corresponding to the region of depleted $\mathrm{HNO}_{3}$ and $\mathrm{H}_{2} \mathrm{O}$. The highest PSC frequencies appear above and below these relative minima near the vortex center, as well as closer to the vortex edge, matching reasonably well with the location of minima in the distribution of $T-T_{\mathrm{NAT}}$.

\subsubsection{Investigation of Multi-Decadal Trends}

In this section, we will investigate the possibility of multi-decadal trends in PSC occurrence by presenting comparisons of appropriately subsampled CALIOP PSC data with the SAM II solar occultation PSC occurrence record from 1978-1989 (Poole and Pitts, 1994) and with ground-based lidar PSC observational record stretching back to the late 1980s and early 1990s.

\subsubsection{Comparison of CALIOP and SAM II PSC Data Records}

Pitts et al. (2018) compared 2006-2017 CALIOP PSC sighting frequencies with SAM II solar occultation PSC sighting frequencies from 1978-82 and 1987-89 (Poole and Pitts, 1994), where the years 1983-86 were excluded to avoid the masking influence of the 1982 El Chichón volcanic eruption. CALIOP 532-nm $\beta_{\text {particulate }}$ data were subsampled to the nominal SAM II solar occultation latitudinal sampling pattern, averaged over $230 \mathrm{~km}$ along track $\times 1 \mathrm{~km}$ vertical bins to mimic the occultation measurement volume, and converted to equivalent $1-\mu \mathrm{m}$ particulate extinction $\left(\alpha_{\text {particulate }}\right)$ values using model relationships from Gobbi et al. (1995). Furthermore, SAM II points were excluded that fell below the effective CALIOP detection threshold, and CALIOP data were excluded that were above the effective maximum optical depth limit of SAM II. Figure 24 shows the time series of multi-year (2006-2017) mean CALIOP-SO (where SO denotes sub-sampling at SAM II solar occultation latitudes) and SAM II PSC column occurrence frequencies (occurrence frequency in 2-km layers summed between 14 and $30 \mathrm{~km}$ altitude) in 10-day bins for the Antarctic (a) and Arctic (b), along with 1- $\sigma$ uncertainties in the sample means (which are a measure of natural variability). In the Antarctic, the magnitude and variability of the CALIOP and SAM II PSC column occurrence frequencies are similar, suggesting there have not been any significant changes since the SAM II era.

The CALIOP and SAM II Arctic records are very similar for the month of February. However, CALIOP mean occurrence frequencies for January and December are significantly higher at the 1- $\sigma$ and 2- $\sigma$ confidence level, respectively, than those observed by SAM II, suggesting there has been a positive trend in the Arctic since the 1978-1989 period. This Arctic early winter change requires an explanation, provided the treatment of both datasets described above establishes a useful comparison. PSC occurrence changes may have a number of reasons, such as changes in ambient temperatures, in precursor gas abundances, or more subtle meteorological changes, e.g. vortex symmetry (strength of a wave-1 pattern suppressing the nucleation of large NAT particles). Of these factors, temperature changes have the strongest

This article is protected by copyright. All rights reserved. 
impact on PSCs. In Figure 24c, we compare the volume fraction of temperatures below $T_{\mathrm{NAT}}$ for the two periods, based on ERA-Interim reanalysis data in a layer from $380 \mathrm{~K}$ to $900 \mathrm{~K}$ on 10 -day intervals at time-dependent latitudes between $65^{\circ} \mathrm{N}$ and $83^{\circ} \mathrm{N}$ determined from the nominal SAM II sampling pattern. There is an increase in this quantity at the $1-\sigma$ confidence level during December from the SAM II period to the CALIOP period, but no discernable increase during January. We hypothesize that early winter temperature changes are indeed responsible for the more statistically significant derived change in December PSC occurrence over the 30-year time span. While it is well known that rising greenhouse gas (GHG) concentrations lead to stratospheric cooling (WMO, 2018), the polar regions are generally thought not to undergo this cooling trend, because the acceleration of the Brewer-Dobson circulation-also related to climate change - counteracts the GHG-induced cooling. However, Langematz et al. (2014) found in a Chemistry-Climate Model (CCM) study that rising GHG concentrations lead to a cooling of the Arctic lower stratosphere in early winter, whereas their model did not show any significant temperature changes in late winter or spring. Further work will be required to confirm whether these arguments provide a reliable basis to explain the observed PSC changes.

\subsubsection{Comparison of CALIOP and Ground-Based Lidar Records}

Ground-based lidars provide quasi-continuous stratospheric measurements at single locations, and time series stretching back more than two decades are available from the McMurdo and Dumont d'Urville Antarctic stations and the Ny-Ålesund station in the Arctic, enabling the investigation of multi-decadal trends in PSC occurrence. For example, David et al. (2010) reported a positive but not statistically significant (+3.2 $\pm 5.4 \% /$ decade) trend in Antarctic winter-long PSC occurrence frequency at Dumont d'Urville from 1989-2008. In this section, we present season-long (June-September for the Antarctic, December-February for the Arctic) CALIOP PSC occurrence statistics over the 2006-2018 period for measurements obtained within $\pm 1^{\circ}$ latitude and $\pm 3.5^{\circ}$ longitude of the McMurdo, Ny-Ålesund, and Dumont d'Urville stations. We compare these to newly compiled PSC occurrence statistics from the McMurdo (JuneSeptember, 1989-2010) and Ny-Ålesund (December-February, 1990-2017) ground-based lidars and published record from Dumont d'Urville (David et al., 2010) to examine for potential multidecadal trends. The PSC occurrence frequency here is defined as the number of days during the season that a PSC was detected at any altitude (12 to $25 \mathrm{~km}$ for McMurdo, tropopause $+1 \mathrm{~km}$ to $28 \mathrm{~km}$ for Dumont d'Urville, 14 to $25 \mathrm{~km}$ for Ny-Ålesund) divided by the total number of measurement days for each.

Figure 25a shows the 1990-2018 time series for McMurdo, where the error bars are 2- $\sigma$ uncertainties of the sample means (representing the natural variability). Season-long PSC sighting frequencies at McMurdo have generally ranged between 0.6-0.9, with no discernable multi-decadal trend. Figure 25b shows the 1989-2018 time series for Dumont d'Urville, where sighting frequencies have generally ranged between $0.1-0.6$. Such lower overall sighting frequencies are expected since Dumont d'Urville is located near the edge of the Antarctic vortex. The CALIOP data subsampled at Dumont d'Urville shows no indication of the small positive trend reported by David et al. (2010). Figure 25c shows the 1995-2018 time series for NyÅlesund, where sighting frequencies have generally ranged between 0.1-0.5, with more variability from year to year than seen at either of the two Antarctic stations, as expected. There

This article is protected by copyright. All rights reserved. 
is very good agreement between the ground-based and CALIOP records at Ny-Ålesund during the 2008-2017 overlap period, and there has been no discernable multi-decadal trend.

\section{Table 3: Summary of Section 2}

- Spaceborne data record of vortex-wide PSC coverage and composition for both hemispheres is available from 2002 to present.

- CALIOP and scaled MIPAS PSC coverages are consistent; scaling may be indicative of PSC patchiness, with Arctic being patchier than the Antarctic.

- Combined analysis of CALIOP and MLS show that STS and ice PSCs occur near thermodynamic equilibrium; NAT mixture PSCs show bimodality, one mode near $T_{\mathrm{NAT}}$ for long exposure time to temperatures below $T_{\mathrm{NAT}}$, one near $T_{\mathrm{STS}}$ for short exposure times and kinetically limited $\mathrm{HNO}_{3}$ uptake on NAT.

- CALIOP, MIPAS, and MLS show consistency in PSC composition for homogeneous cloud scenes.

- Antarctic PSC coverage is similar from year-to-year, but each year in the Arctic is unique.

- Unprecedented vortex-wide climatological views of PSC occurrence and composition reveal variation in composition with season, altitude, and spatial position; tying in with changes in $\mathrm{HNO}_{3}$ and $\mathrm{H}_{2} \mathrm{O}$ caused by denitrification and dehydration.

- In the Antarctic, PSC occurrence is very similar between the SAM II (1978-89) and CALIOP (2006-2017) eras; in contrast, there has been a significant increase in Arctic PSC occurrence in December and January, possibly in response to early winter cooling related to climate change.

\section{Formation Pathways and Particle Characteristics}

As discussed in Sections 1 and 2, PSCs are predominantly external mixtures of different particle compositions whose main constituents are water $\left(\mathrm{H}_{2} \mathrm{O}\right)$ and nitric acid $\left(\mathrm{HNO}_{3}\right)$, which have formed on or evolved from stratospheric sulfuric acid aerosol (SSA) droplets. Based on the PSC spatial and temporal distributions and particle composition described in Section 2, the fundamental understanding of PSC formation pathways has advanced considerably during the last two decades. The process of condensational growth of liquid STS droplets is well understood and discussed here only briefly. The processes by which solid PSC particles - primarily NAT and ice - form were noted to be the least well understood in recent reviews of PSC science (Lowe and MacKenzie, 2008; Peter and Grooß, 2012). Based on laboratory experiments, there is no doubt that ice can nucleate homogeneously in STS droplets (Koop et al., 2000), whereas the homogeneous nucleation of NAT is kinetically suppressed (Koop et al., 1995). Homogeneous nucleation of ice in cirrus clouds and PSCs has been confirmed by field observations (e.g. Figure 3 in Koop et al., 2000). However, in recent years evidence has been growing for the existence of a heterogeneous nucleation pathway of solid PSC particles on preexisting foreign nuclei. This is

This article is protected by copyright. All rights reserved. 
based on satellite (Pitts et al., 2011) and in-situ observations (Weigel et al, 2014, Ebert et al., 2016), modeling work (Hoyle et al., 2013; Engel et al., 2013) and additional laboratory studies (James et al., 2018). We will present a comprehensive picture of PSC formation pathways emerging from the new studies, sorted by particle compositions as illustrated in Figure 26.

\subsection{Growth of STS Droplets (Figure 26, Pathway 1)}

The stratospheric background aerosol, which consists of SSA droplets, is globally distributed (Junge et al., 1961b). Under volcanically quiescent conditions, the SSA typically has a lognormal size distribution with a mode radius around $0.07 \mu \mathrm{m}$. Upon cooling, the SSA droplets start to grow by uptake of additional $\mathrm{H}_{2} \mathrm{O}$, as a consequence of the decreasing water vapor pressure of the $\mathrm{H}_{2} \mathrm{SO}_{4}-\mathrm{H}_{2} \mathrm{O}$ solutions (Carslaw et al., 1997). A rapid change in particle composition takes place at around $T_{\text {ice }}+4 \mathrm{~K}$, when the droplets have become sufficiently dilute so that small amounts of $\mathrm{HNO}_{3}$ can condense into the acidic liquid. Around $T_{\text {ice }}+3 \mathrm{~K}$, the weight percentage of $\mathrm{HNO}_{3}$ in the droplets exceeds that of $\mathrm{H}_{2} \mathrm{SO}_{4}$ (Carslaw et al., 1994; Figure $27 \mathrm{~b}$ ). This temperature is called $T_{\mathrm{STS}}$, below which particle volume increases significantly.

STS droplet mode radii increase to around $0.3 \mu \mathrm{m}$ after all the $\mathrm{HNO}_{3}$ has partitioned from the gas phase to the condensed phase. Figure 27 illustrates airborne measurements and thermodynamic electrolyte model calculations of SSA and STS PSCs. Carslaw et al. (1994) demonstrated that airborne measurements by Dye et al. (1992) can be modeled when the thermodynamics of the ternary solutions are properly taken into account. This determines the uptake of $\mathrm{HNO}_{3}$, leading to the observed growth of the STS droplets when temperature decreases. Uptake of $\mathrm{H}_{2} \mathrm{O}$ alone cannot explain the measurements since particle volumes of $\mathrm{H}_{2} \mathrm{SO}_{4} / \mathrm{H}_{2} \mathrm{O}$ solution droplets would remain too small (dotted line, panel (a)). Also, the volume of solid NAT particles does not agree with the observations since nucleation of NAT particles without a kinetic barrier would produce a sharp onset of particle growth immediately below $T_{\mathrm{NAT}}$ (dashed line, panel (a)).

Sulfuric acid tetrahydrate (SAT) particles are the thermodynamically stable form of $\mathrm{H}_{2} \mathrm{SO}_{4} / \mathrm{H}_{2} \mathrm{O}$ mixtures over a wide range of stratospheric conditions (Middlebrook et al., 1993; Koop and Carslaw, 1996). However, laboratory experiments have reported that solutions of $\mathrm{H}_{2} \mathrm{SO}_{4}$ at $195 \mathrm{~K}$ require tempering for days to achieve crystallization as a hydrate (Hülsmann and Biltz, 1934; Luo et al., 1994; and references therein). Direct observational evidence to date that SAT particles exist in the atmosphere is lacking or questionable. Correspondingly, the weak but systematic increase in particle volume at $T>T_{\text {NAT }}$ in Figure 27 a has been interpreted as $\mathrm{H}_{2} \mathrm{O}$ uptake by liquid SSA in the absence of SAT. The existence of SAT has been inferred indirectly based on lidar observations (e.g., Beyerle et al., 2001) of non-spherical particles at ambient temperatures $2 \mathrm{~K}$ above $T_{\mathrm{NAT}}$. However, temperatures in this study were obtained from daily radio soundings, and nominal volume mixing ratios were assumed for $\mathrm{H}_{2} \mathrm{O}(5 \mathrm{ppmv})$ and $\mathrm{HNO}_{3}$ (10 ppbv), simplifications that weaken this conclusion. Zhu et al. (2015) point out that SAT deliquesces when temperature decreases and NAT supersaturation increases, and that nucleation of NAT on SAT requires supercooling by up to $8 \mathrm{~K}$ as shown by laboratory measurements (Iraci et al., 1995) and calculations (MacKenzie et al., 1995). Therefore, even if SAT particles existed in the atmosphere, they might be irrelevant for PSC formation and therefore are not discussed further here. We will return to the nucleation mechanism when discussing the presence of refractory heterogeneous nuclei in the STS particles in Section 3.3.

This article is protected by copyright. All rights reserved. 


\subsection{Characteristics of NAT Particles}

Mixtures of liquid droplets and NAT particles are the dominant PSC composition class observed at most altitudes in both the Antarctic and Arctic (see Section 2.3.2). Hanson and Mauersberger (1988) showed that under typical stratospheric conditions NAT is the stable hydrate of nitric acid and that it can exist at temperatures up to $7 \mathrm{~K}$ above $T_{\text {ice. }}$ In January 2000, the first direct observations of NAT particles on board a balloon gondola above Kiruna, Sweden, were made. A molar ratio of 3:1 between water and nitric acid was measured, confirming the existence of solid crystalline NAT at temperatures above $T_{\text {ice }}$ (Voigt et al., 2000).

There is continuing discussion about the existence of metastable hydrates such as NAD. Laboratory studies indicate the possible existence of NAD under polar stratospheric conditions (Worsnop et al., 1993; Möhler et al., 2006; Stetzer et al., 2006; Grothe et al., 2008). Furthermore, spectroscopically different configurations of NAT and NAD have been identified, namely $\alpha-$ NAT and the more stable $\beta$-NAT, as well as $\alpha$-NAD and $\beta$-NAD (e.g. Tizek et al., 2002, 2004). However, unlike NAT, no evidence has been found for NAD in the stratosphere (Höpfner et al., 2006a). For this reason, we focus on NAT particles, even though nitric acid hydrates other than NAT cannot be excluded under certain conditions.

Since the reviews by Lowe and MacKenzie (2008) and Peter and Grooß (2012), there have been new research on NAT particle shapes. Whereas previously NAT particles have been described as medium-sized (radii $\geq 1 \mu \mathrm{m}$ ) solid, slightly aspherical particles, Molleker et al. (2014) and Woiwode et al. $(2014 ; 2016 ; 2019)$ suggest that NAT particles may be highly aspherical. The following section summarizes findings made from in situ and remote sensing particle measurements in the last decade.

\subsubsection{Evidence for Highly Aspherical $\beta$-NAT Particles}

The RECONCILE (von Hobe et al., 2013) and ESSenCe (Kaufmann et al., 2015) field campaigns in the Arctic winters 2009/10 and 2011/2012 included flights of the Russian highaltitude aircraft M55 Geophysica (Stefanutti et al., 1999). The in situ measurements by Optical Particle Counters (OPCs) and a total $\mathrm{NO}_{\mathrm{y}}$ (the sum of all reactive nitrogen oxides) instrument onboard the Geophysica extended previous spatially limited observations of large $\mathrm{HNO}_{3}$ containing particles with diameters exceeding $20 \mu \mathrm{m}$ (Goodman et al., 1997; Fahey et al., 2001; Brooks et al., 2003) to synoptic scales (Molleker et al., 2014). Apparent particle diameters of up to $30 \mu \mathrm{m}$ and large amounts of condensed $\mathrm{HNO}_{3}$ inferred from these observations cannot be explained with established NAT particle growth scenarios and available condensable $\mathrm{HNO}_{3}$, as sedimentation speeds of such particles would not give them enough time to grow and the condensed $\mathrm{HNO}_{3}$ would exceed the amount present in the corresponding layers of the stratosphere . Collocated infrared limb observations by the airborne MIPAS-STR (Michelson Interferometer for Passive Atmospheric Sounding - STRatospheric aircraft) from the Geophysica confirmed the presence of the $\beta$-NAT phase (e.g. Tizek et al., 2004) and suggest highly aspherical NAT particle shapes (Woiwode et al., 2016).

FSSP (Forward Scattering Spectrometer Probe) and CDP (Cloud Droplet Probe) measurements make use of the forward scattering of laser light by the particles (Molleker et al., 2014). While uncertainty in particle shape and refractive index limit the accuracy of particle sizing, the detected particle number densities are quite precise. Unlike with other measurements in tropospheric cirrus clouds, artifacts affecting particle number densities such as shattering were

This article is protected by copyright. All rights reserved. 
not found in the PSC data. Examples of volume equivalent size distributions (in diameter) measured in NAT-containing PSCs are shown in Figure 28a,b (black lines). In situ measurements of $\mathrm{NO}_{\mathrm{y}}$ involved a forward-facing inlet with a capability of detecting low particle number densities down to $10^{-6} \mathrm{~cm}^{-3}$ (Molleker et al., 2014). Surprisingly, the forward $\mathrm{NO}_{\mathrm{y}}$ signal (sensitive to the sum of gas phase and condensed $\mathrm{HNO}_{3}$ ) during RECONCILE was saturated in many cases due to very high levels of ambient $\mathrm{NO}_{\mathrm{y}}$ during most prominent encounters of NATcontaining PSCs. A corresponding volume equivalent size distribution measured during RECONCILE is shown in Figure 28a (red line). The histogram in Figure 28c summarizes particle number densities representative of NAT measured by the OPCs. Notably, there is a sharp upper limit of NAT particle number density occurrence at $10^{-2} \mathrm{~cm}^{-3}$, with most of the population in the range between $10^{-3}$ to $10^{-2} \mathrm{~cm}^{-3}$. A large NAT particle mode, roughly encompassing particles with diameters greater than $12.5 \mu \mathrm{m}$, shows number densities largely between $10^{-4}-10^{-}$ ${ }^{3} \mathrm{~cm}^{-3}$. A new feature of the latter distribution is the "tail" to higher number densities of up to about $0.5 \times 10^{-2} \mathrm{~cm}^{-3}$. The corresponding gas phase equivalent $\mathrm{HNO}_{3}$ exceeds by about one order of magnitude the value of 1.5 ppbv inferred by Fahey et al. (2001) from Arctic winter 1999/2000 measurements and found in simulations (Grooß et al., 2014). This raises the question as to whether enough condensable $\mathrm{HNO}_{3}$ is available to generate such large particles in the number densities measured. Large (presumably) NAT particles of similar sizes but lower number densities are reported in various publications (Dye et al., 1992, Goodman et al., 1997, Fahey et al., 2001, Brooks et al., 2003).

The issue of large particle sizes and condensable $\mathrm{HNO}_{3}$ amounts was further explored in conjunction with MIPAS-STR infrared limb observations during ESSenCe (Figure 29a). These remote measurements exhibit a spectral signature around $820 \mathrm{~cm}^{-1}$ and an overall spectral pattern compatible with large highly aspherical $\beta$-NAT particles (Woiwode et al. 2016). The measurements were performed inside the lower region of a PSC near $T_{\mathrm{NAT}}$. The collocated in situ particle observations (Molleker et al., 2014) were used to constrain the size distribution (Figure $29 b)$. Mie calculations of spherical $\alpha / \beta-N A T$, NAD, STS and ice particles according to Höpfner et al. (2006a) could not satisfactorily reproduce the observed spectral signature (for spherical $\beta$ NAT particles see red and blue spectra in Figure 29a). In contrast, T-Matrix calculations using the approach of Bi et al. (2013) assuming randomly oriented highly aspherical $\beta$-NAT particles (aspect ratios 0.1 or 10 for elongated or disk-like spheroids, respectively) reproduced the MIPAS-STR observations to a large degree (green spectrum in Figure 29a). Assumptions on particle shapes were motivated by a study by Grothe et al. (2006), who observed highly aspherical $\beta$-NAT in laboratory experiments. The signature at $820 \mathrm{~cm}^{-1}$ is similar to the "peaklike" signature of small $\beta$-NAT particles with high volume densities reported by Spang and Remedios (2003) and Höpfner et al. (2006a), but shows a red-shifted "step-like" shape along with an overall flat spectral baseline towards higher wave numbers. Best agreement was found after scaling the size distribution constrained by the in situ measurements to $20 \%$ smaller sizes (Figure 29b, blue line). In particular, assuming a lognormal size distribution with a median radius $(r)$ of $4.8 \mu \mathrm{m}$ (radius of volume-equivalent sphere), a mode width $(\sigma)$ of 1.35 , and a number density $(n)$ of $1.5 \times 10^{-3} \mathrm{~cm}^{-3}$ for the main mode enabled the most accurate modeling of the observed spectral signature.

We can only speculate on the mechanisms leading to the formation of these highly aspherical, large NAT particles, differing significantly from previous observations and model simulations assuming spherical or weakly aspherical (aspect ratios around 0.9) NAT particles. Particle growth is likely to start at a nearly spherical geometry immediately after nucleation,

This article is protected by copyright. All rights reserved. 
which takes place in a spherical droplet. However, the crystalline particles may acquire higher asphericities when growing to large sizes in mildly supersaturated vapors, making the particle growth more preferable in locations surrounded by less depleted vapor mixing ratios, i.e. close to the tips and far from the center of the crystal. The larger particle sizes suggested by the optical in situ observations (Figure 28) might be explained by an instrument broadening effect when randomly oriented aspherical particles enter the laser beam in combination with a slight overestimation of the particle sizes. The simulations of the MIPAS-STR spectra may still include significant uncertainties, and issues remain in the comparison with the $\mathrm{NO}_{\mathrm{y}}$ observations. Therefore, the growth habits and precise shapes of large NAT particles remain critical parameters for reconciling airborne in situ and remote sensing observations. Furthermore, a variability of particle shapes and habits might be present in NAT PSCs due to different nucleation and growth histories. A growth of NAT layers on ice particles was proposed by Biermann et al. (1998), and hollow NAT shells with low $\mathrm{HNO}_{3}$ content might remain after short growth times and evaporation of the ice core particles (see Goodman et al., 1997). The size distribution associated with the measurements in Figure 29 corresponds to a still very high amount of $\sim 9$ ppbv of gas phase equivalent $\mathrm{HNO}_{3}$, which can only be explained by particles falling slowly and collecting high amounts of $\mathrm{HNO}_{3}$.

Recently, the baseline of the spectrum (i.e. when trace gas signatures are neglected, see Woiwode et al., 2019, Figure 1) from 817.5 to $961 \mathrm{~cm}^{-1}$ was utilized to define a simple detection method sensitive to highly aspherical $\beta$-NAT populations with volume-equivalent median radii of 3-7 $\mu \mathrm{m}$. Underlying size distributions are compatible with in situ observations of large $\mathrm{HNO}_{3}-$ containing particles reported in the literature (compare Molleker et al., 2014, references therein) and available $\mathrm{HNO}_{3}$. Vortex-wide application of the method to MIPAS observations in the Arctic 2011/12 PSC season resulted in positive detections in regions close to $T_{\mathrm{NAT}}$ and above $T_{\text {ice }}$, and were consistent with collocated gas-phase $\mathrm{HNO}_{3}$ sequestration seen by MLS. Thereby, the combination of MIPAS and MLS observations showed unexpectedly fast population growth and gas-phase $\mathrm{HNO}_{3}$ consumption along with long persistence of observed populations.

\subsubsection{Nucleation of NAT Particles}

For the nucleation of NAT, several pathways have been discussed to date. They are described in detail by Peter and Grooß (2012). The most relevant aspects of these possible nucleation mechanisms are summarized in the following sections.

\subsubsection{Kinetic Suppression of Homogeneous NAT Nucleation}

Homogeneous nucleation of NAT particles from STS droplets was first postulated by Molina et al. (1993), but Koop et al. (1995) disproved this idea by showing that bulk samples of ternary solutions can be supercooled to temperatures below $T_{\text {ice }}$ under stratospheric conditions without freezing. A high nucleation barrier renders homogeneous freezing of NAT out of liquid aerosol droplets impossible. Alternative proposals were made about homogeneous nucleation of glassy aerosols (Tabazadeh et al., 1995) or interface-induced homogeneous nucleation (Tabazadeh et al., 2002), where NAT nucleation happens "pseudo-heterogeneously" at the airsolution interface of the droplet. However, both mechanisms have been shown to be inefficient in the stratosphere. Koop et al. (1997a) showed that the existence of an amorphous state is unlikely under stratospheric conditions and that crystal growth at stratospheric temperatures is fast enough to lead to rapid crystallization of a droplet once NAT nucleated. Knopf (2006) evaluated

This article is protected by copyright. All rights reserved. 
the surface-induced nucleation of NAT or NAD in $\mathrm{HNO}_{3}$-containing particles and showed it to be insufficient to explain stratospheric observations. The nucleation of NAT has not been observed in laboratory experiments and the nucleation of NAD in cloud chamber experiments obtained particle number densities of $6 \times 10^{-6} \mathrm{~cm}^{-3}$ (Möhler et al., 2006), which are at least two orders of magnitude lower than low number density NAT mixtures inferred from CALIOP and MIPAS. This means that NAT needs to either nucleate heterogeneously on ice, which has been shown to be efficient, e.g. downstream of mountain wave ice clouds, or requires another independent NAT nucleus. In Figure 26, homogeneous NAT nucleation would correspond to pathway 2 without requiring a heterogeneous nucleus. However, SSA, upon which all PSC particles form, has a significant refractory component (Curtius et al., 2005; Weigel et al., 2014). Given the evidence against homogeneous NAT nucleation, we prefer to think of pathway 2 as being triggered by suitable heterogeneous nuclei in the present review (see Section 3.2.2.3).

\subsubsection{Heterogeneous NAT Nucleation on Preexisting Ice (Figure 26, Pathway 6)}

There is indisputable evidence for heterogeneous nucleation of NAT particles on preexisting ice particles. As mentioned previously, Koop et al. (1997a) showed that crystal growth at stratospheric temperatures is fast enough to lead to rapid crystallization of an STS droplet after NAT nucleation. Deposition nucleation of NAT on bare ice surfaces was suggested to be the most likely nucleation mechanism (Biermann et al., 1998). In an attempt to model this mechanism, Luo et al. (2003) suggested that extreme NAT supersaturations in mountain wave ice PSCs promote NAT nucleation and developed a parameterization for deposition nucleation of NAT on ice. This is supported by observations of NAT PSCs downstream of mountain wave ice clouds (e.g. Carslaw et al., 1998a,b; Wirth et al., 1999). Spaceborne measurements by MIPAS, MLS, and CALIOP led to the discovery of an Antarctic circumpolar belt of NAT PSCs triggered by localized cooling events with ice clouds in mountain waves (Höpfner et al., 2006b; Lambert et al., 2012; Section 4.2). While these NAT PSCs have high number densities up to $0.1-1 \mathrm{~cm}^{-3}$ (Voigt et al., 2003), the origin of PSCs with much lower particle number densities remained unclear for a long time. Fueglistaler et al. (2002a,b) developed the idea of a "mother cloud" as source for low number density NAT particles, which may grow to large sizes, sometimes called "NAT rocks". The largest NAT particles with number densities of about $10^{-4} \mathrm{~cm}^{-3}$ fall out of the NAT mother cloud into undepleted, supersaturated air and may grow to radii of around $10 \mu \mathrm{m}$ some kilometers below the mother cloud. This mechanism can partly explain some lidar measurements (Biele et al., 2001; Pitts et al., 2007, 2009) as well as denitrification, which is caused predominantly by NAT particles in small number densities (Waibel et al., 1999). Yet, iceinduced NAT nucleation cannot fully explain denitrification, because ice nucleation requires temperatures to be typically $7-10 \mathrm{~K}$ below $T_{\mathrm{NAT}}$, which occur only rarely in the Arctic (Carslaw et al., 1999). The contribution of stratospheric mountain waves to the formation of large NAT particles and subsequent denitrification of the Arctic polar vortex has been investigated by Mann et al. (2005) using the 3-D DLAPSE (Denitrification by Lagrangian Particle Sedimentation) model. They concluded that NAT particles that have sedimented from the mother cloud bases can occupy more than $60 \%$ of the NAT supersaturated region. Therefore, they represent an efficient pathway to irreversible denitrification, revealing that "denitrification due to this mechanism could potentially be responsible for as much as $80 \%$ of that observed" (Mann et al., 2005). Nevertheless, more and more subsequent observations indicated the need for an additional heterogeneous nucleation mechanism for NAT particles in the absence of any ice.

This article is protected by copyright. All rights reserved. 


\subsubsection{Heterogeneous NAT Nucleation on Foreign Nuclei (Figure 26, Pathway 2)}

It is well-known that tropospheric cirrus clouds can form after homogeneous nucleation of ice, but heterogeneous freezing is also a well-accepted pathway (e.g. Rogers and Yau, 1989; Pruppacher and Klett, 1997). The possibility that there is a similar pathway for PSC formation has been discussed recently, although the stratosphere is arguably much cleaner and has fewer heterogeneous nuclei than the troposphere. Various studies provided evidence that heterogeneous nucleation of NAT on ice particles generated in regions of mountain-wave activity might not be the sole mechanism for some of the observed NAT PSCs (e.g. Pagan et al., 2004; Voigt et al., 2005). Drdla et al. (2002) discussed different NAT freezing processes and concluded that reproduction of key characteristics of the observed PSCs in the Arctic winter 1999/2000 requires a freezing process that occurs above $T_{\text {ice. }}$. Further evidence for an ice-free NAT nucleation pathway came from measurements during the EUPLEX Arctic campaign in 2003, showing small NAT particles in air masses originating from a warm region with $T>T_{\mathrm{NAT}}+10 \mathrm{~K}$ only 36 hours before the measurement, subsequently cooling to less than $3 \mathrm{~K}$ below $T_{\mathrm{NAT}}$ and $4 \mathrm{~K}$ above $T_{\text {ice }}$. For this case, Voigt et al. (2005) derived an air-volume-averaged NAT nucleation rate and speculated that the heterogeneous NAT nucleation might have occurred on meteoritic material. The suitability of meteoritic material as a possible NAT nucleus had been documented earlier in the laboratory work of Bogdan et al. (1999, 2003). Recently, Ward et al. (2014) presented the first NAT nucleation rate measured in situ by particle sensors on a balloon-borne gondola floating in an air parcel at temperatures below $T_{\mathrm{NAT}}$, but always above $T_{\text {ice. }}$.

The availability of global satellite observations provided compelling evidence that icefree heterogeneous NAT nucleation is regular and widespread. First, CALIOP observations showed that the early season of the Arctic winter 2009/2010 was characterized by patchy, tenuous PSCs composed of primarily low number density liquid/NAT mixtures. Ice PSCs were not detected by CALIOP during this period anywhere in the vortex, MLS showed no sign of dehydration, and temperatures (obtained from high-resolution analyses) remained well above $T_{\text {ice. }}$ Consequently, Pitts et al. (2011) concluded that the observed early season NAT mixture PSCs must have formed through a wide-spread non-ice nucleation mechanism. Subsequently, Hoyle et al. (2013) took CALIOP observations and developed a detailed microphysical parameterization for NAT nucleation based on laboratory results on Arizona Test Dust (Marcolli et al., 2007). Grooß et al. (2014) used this saturation-dependent parameterization for NAT nucleation to replace the former, constant NAT nucleation rate (Grooß et al., 2005) within the Chemical Lagrangian Model of the Stratosphere (CLaMS). Lambert et al. (2016) analyzed MLS and CALIOP data from 2006 through 2015 and confirmed that the initiation of NAT nucleation and the subsequent development of large NAT particles capable of sedimentation and denitrification in the early winter do not emanate from an ice-seeding process. Very recently, Tritscher et al. (2019) demonstrated that the new parameterization in CLaMS accurately reproduces PSC observations in the beginning and the overall denitrification at the end of the 2009/2010 Arctic winter and the 2011 Antarctic winter. Major properties of the heterogeneous NAT nuclei are discussed in the following subsection.

\subsection{The Possible Origin of Heterogeneous NAT Nuclei}

Meteoritic particles have been proposed to serve as ice and/or NAT nuclei (Bogdan et al., 1999,2003 ), and the availability of meteoritic material in the stratosphere and its quality to trigger cloud formation are the subject of present research. There is considerable uncertainty in

This article is protected by copyright. All rights reserved. 
not only the composition, but also number densities and sizes of interplanetary dust particles (Plane, 2012). With an average extraterrestrial mass influx of 20 to 100 tons per day (Cziczo et al., 2001), which compares with 160 tons per day of sulfur influx from the troposphere (or 650 tons per day of aqueous sulfuric acid) during volcanically quiescent times (Thomason and Peter, 2006), meteoritic material constitutes 3 to 15 percent by weight of the stratospheric aerosol. It is spread globally and funneled into the polar winter stratosphere of both hemispheres by the Brewer-Dobson circulation (Engel et al., 2013). Recent in situ instrumentation can distinguish various types of aerosol particles in the stratosphere and determine their relative abundance. In the background lower stratosphere, a large fraction of SSA droplets contains inclusions of meteoritic and/or organic material (Murphy et al., 2014; Schneider et al., 2021). The solid inclusions could have important implications for the ability of particles to act as freezing nuclei for PSCs. During several airborne campaigns, Weigel et al. (2014) conducted in situ measurements of SSA. They measured with a condensation particle counter (CPC) at altitudes up to $20 \mathrm{~km}$ on board the aircraft M-55 Geophysica from Kiruna, Sweden, in January through March 2010 (RECONCILE), as well as in December 2011 (ESSenCe). Particle concentrations as well as the fraction of non-volatile particles were measured, as shown in Figure 30. Up to eight out of eleven particles $\mathrm{cm}^{-3}$ were found to contain thermo-stable (at $250{ }^{\circ} \mathrm{C}$ ) residuals with diameters in the range $10 \mathrm{~nm}-1 \mu \mathrm{m}$. These results confirm earlier Arctic measurements by Curtius et al. (2005), who measured similar vertical profiles in January through March 2003 (EUPLEX). Further, Curtius et al. (2005) also found a higher fraction of nonvolatile compounds inside $(67 \%)$ the vortex than outside (24\%). Those findings support a funneling effect of the winter vortex with air masses subsiding inside the winter vortex from the upper stratosphere and mesosphere, thus enabling transport of refractory particles into the lower polar stratosphere. Similar non-volatile particle fractions were found by Campbell and Deshler (2014) in balloonborne stratospheric condensation nuclei measurements in the Antarctic polar vortex in late winter.

During RECONCILE, samples of aerosol particles with diameters larger than about $10 \mathrm{~nm}$ were collected with a dual-stage cascade impaction system (Ebert et al., 2016). Fe-rich particles, Ca-rich particles, silicates, silicate/carbon mixed particles and mixed metal particles were identified. They originate most likely from different sources, such as meteoric material and space debris and to a lesser extent terrestrial sources (Ebert et al., 2016, Bogdan et al., 1999). During PSC events, the number of large (>500 $\mathrm{nm}$ ) refractory particles decreases. The most likely explanation for this behavior is that large refractory particles are good nuclei for larger (aerodynamic diameter $>1 \mu \mathrm{m}$ ) PSC particles. Such larger PSC particles suffer high transmission losses in the forward sampling (aircraft-borne) inlet and inlet tubing; hence their refractory inclusions do not reach the impactor substrates. Ebert et al. (2016) reported 90\% transmission and sampling for aerodynamic particle diameters $<1 \mu \mathrm{m}$, but $30-40 \%$ for $4 \mu \mathrm{m}$ particles and only $5 \%$ for $6 \mu \mathrm{m}$ particles. Large refractory particles have been observed in impactor samples collected under similar stratospheric conditions in PSC-free regions. Schütze et al. (2017) continued the analyses of the chemical composition, size and morphology of the refractory particles by scanning electron microscopy and energy-dispersive X-ray microanalysis for more particle samples collected in the polar stratosphere during SOLVE. They concluded that "recondensed organic matter and extraterrestrial particles, potentially originating from ablation and fragmentation, were found as possible sources of the refractory carbonaceous particles studied."

This article is protected by copyright. All rights reserved. 
Besides these field measurements, laboratory studies have been performed on the NAT nucleation efficacy of meteoritic particles. Biermann et al. (1996) used micrometeorites collected in Antarctica and immersed them in supercooled ternary bulk solutions to determine heterogeneous NAT freezing rates. They concluded that micrometeorites would be unsuitable for explaining observed NAT PSCs. However, their intent was to explain NAT number densities of $10 \mathrm{~cm}^{-3}$ as they occur only in the densest "enhanced NAT mixture" PSCs, resulting in an upper limit for the nucleation rate corresponding to an atmospheric freezing time of 20 months. Bogdan et al. $(1999,2003)$ used a different approach with nanometer-scale aerosol samples, concluding that meteoritic smoke silica particles can indeed induce heterogeneous freezing of $\mathrm{HNO}_{3}$ hydrates at temperatures above $T_{\text {ice. }}$. Voigt et al. (2005), also pointing to the studies by Bogdan et al. (1999, 2003), argued that the long freezing times derived by Biermann et al. (1999) would be much shorter if NAT particle number densities $\left(n_{\mathrm{NAT}}\right)$ were much lower. For $n_{\mathrm{NAT}}=10^{-4}-10^{-3}$ $\mathrm{cm}^{-3}$, an exposure to sufficiently low temperatures of less than a day would suffice. This is still regarded as upper limit, since Biermann et al. (1996) could not exclude artifacts caused by heterogeneous nucleation on the test tube walls. Based largely on these arguments, meteoritic material emerged as a suitable trigger for the formation of low number density NAT or synopticscale ice clouds in the stratosphere. This was recently confirmed by James et al. (2018), who showed that proxies for both meteoritic materials, smoke particles (amorphous silica analogues: fumed silica and fused quartz) and meteoritic fragments (ground meteorite analogues), have a capacity to nucleate $\mathrm{HNO}_{3}$ hydrates. In combination with estimates from a global meteorite model, they estimated the amount of material in the polar stratosphere and showed that meteoric material might explain NAT observations in early season PSCs, with NAT nucleation starting at even higher temperatures than estimated by Hoyle et al. (2013). In contrast to the resulting synoptic scale PSCs, the origin of enhanced NAT mixtures and wave ice PSCs is attributed to strong temperature fluctuations, for example related to homogeneous nucleation in small-scale orographic wave events.

\subsection{Formation of Stratospheric Ice Particles}

Water vapor is the most abundant condensable species in the stratosphere. Thus, ice crystals, which must form at temperatures a few degrees below $T_{\text {ice, }}$, generally produce a large backscatter ratio and a significant depolarization in lidar measurements and are therefore easy to detect and distinguish from other types of PSCs. However, simulations of ice particle formation remain challenging, because water equilibrates within the STS solution droplets and in the gas phase on timescales of seconds (Meilinger et al., 1995), meaning that small temperature fluctuations, which are not typically resolved in large-scale model simulations, may suffice to initiate ice particle nucleation. Ice particle number densities are very much dependent on the instantaneous cooling rate of the air parcel in the moment the ice cloud forms (Toon et al., 1989b; Hoyle et al., 2005). Synoptic-scale ice PSCs with number densities of $10^{-3}$ to $10^{-2} \mathrm{~cm}^{-3}$ (Dye et al., 1992) can exhibit large particle sizes. In contrast, the entire background aerosol of $\sim 10 \mathrm{~cm}^{-3}$ can freeze in mountain wave events characterized by high cooling rates and, in this case, ice particle radii remain smaller than about $2 \mu \mathrm{m}$ (Fueglistaler et al., 2003). For this reason, the underlying wind and temperature fields including small-scale temperature fluctuations or appropriate parameterizations thereof are of great importance for the modeling of ice PSCs. The dynamical forcing of PSCs is discussed in detail in Section 4. In this subsection, we summarize the most likely routes of ice formation.

This article is protected by copyright. All rights reserved. 


\subsubsection{Homogeneous Ice Nucleation (Figure 26, Pathway 5)}

Homogeneous nucleation of ice particles from aqueous solutions is a well understood pathway to form ice clouds, in the troposphere as well as in the stratosphere. A theoretical description of homogeneous ice nucleation in aqueous solutions of atmospheric relevance was developed by Koop et al. (2000) based on laboratory freezing experiments. Koop and colleagues also developed a useful formulation for the ice nucleation rate coefficient $J$ as function of $\mathrm{H}_{2} \mathrm{O}$ activity and pressure. They showed that a supercooling of about 3-4 K below $T_{\text {ice }}$ is required to trigger homogeneous ice nucleation, i.e. $T_{\text {HomNuc }} \sim T_{\text {ice }}-4 \mathrm{~K}$ (Koop et al., 1998; Carslaw et al., 1998b). In this formulation, the rate of ice nucleation is proportional to the volume of supercooled water. The formulation of Koop et al. (2000) is used in the majority of present models.

Mountain-wave-induced PSCs are predominantly formed by homogeneous ice nucleation. In the Arctic, 54\% of water ice PSCs are attributed to orographic gravity waves (Alexander et al., 2013) and their colorful appearance is indicative of nearly monodisperse distributions of micrometer-sized ice particles resulting from rapid cooling events (Peter and Grooß, 2012).

\subsubsection{Heterogeneous Ice Nucleation on Preexisting NAT Particles (Figure 26, Pathway 3)}

Around 1990, when explanations for the ozone hole were still in their infancy, the importance of PSCs had been recognized. A simple 3-stage model was developed: first SSA droplets would form solid hydrates (e.g. SAT), next NAT would form via deposition nucleation on the $\mathrm{H}_{2} \mathrm{SO}_{4}$ hydrates, and finally ice would nucleate on NAT, with the processes sorted according to their thermodynamic equilibrium temperatures (Koop et al., 1997b; Peter, 1997; Lowe and MacKenzie, 2008). Not much attention had been paid to this potential sequence after homogeneous ice nucleation had been postulated. However, observations of widespread synoptic-scale ice clouds in the Arctic (Pitts et al., 2011; Voigt et al., 2018) suggested that ice nucleation does occur at supersaturations less than that required for homogeneous ice nucleation, i.e. at temperatures above $T_{\text {HomNuc }} \sim T_{\text {ice }}-4 \mathrm{~K}$. Heterogeneous ice nucleation on preexisting NAT and/or SAT particles is a reasonable pathway, which should not be ignored. Laboratory work by Fortin et al. (2003) suggested that a supercooling of 0.1-1.3 K below $T_{\text {ice }}$ would suffice to nucleate ice heterogeneously on SAT surfaces. However, as mentioned earlier, it is unclear if SAT itself ever forms, given its failure to nucleate even in laboratory experiments using 1:4 solutions of $\mathrm{H}_{2} \mathrm{SO}_{4}: \mathrm{H}_{2} \mathrm{O}$ in contact with solid surfaces. Furthermore, even if it does form, it deliquesces upon approaching $T_{\text {ice. }}$. Although depolarized backscatter signals in lidar measurements above $T_{\mathrm{NAT}}$ have been ascribed to SAT, such signals are weak and hard to interpret. Therefore, the nucleation of ice on SAT is highly uncertain (e.g., Lowe and MacKenzie, 2008; Peter and Grooß, 2012). This is different for the nucleation of ice on NAT particles. Barone et al. (1997) showed by means of FTIR reflection-absorption spectroscopy that ice nucleation on NAT or NAD particles at $185 \mathrm{~K}$ occurred only after $\mathrm{H}_{2} \mathrm{O}$ vapor pressures exceeded a saturation ratio of 1.5 , which would be only marginally above the homogeneous ice nucleation threshold. Khosrawi et al. (2011), Engel et al. (2014) and Voigt et al. (2018) revisited the topic recently with the intent of explaining field observations. Based on reciprocity arguments, they applied NAT-on-ice nucleation rates to ice-on-NAT nucleation rates and suggested ice nucleation on preexisting NAT particles as a possible explanation of recent

This article is protected by copyright. All rights reserved. 
observations. However, more careful analyses and laboratory work are required to quantify this pathway in more detail.

\subsubsection{Heterogeneous Ice Nucleation on Foreign Nuclei (Figure 26, Pathway 4)}

Synoptic-scale temperatures in the Arctic stratosphere rarely fall to $T_{\text {HomNuc. }}$ Yet, in January 2010 and in January 2016, ice PSCs were observed in the Arctic on large spatial scales without temperatures being cold enough to explain ice formation homogeneously (Engel et al., 2013; Voigt et al., 2018). Therefore, heterogeneous ice formation mechanisms, which take effect at higher temperatures, have been invoked as an explanation. Bogdan et al. (2003) showed that fumed silica, possibly representative of meteoritic smoke particles, is suitable for inducing heterogeneous freezing of ice under stratospheric conditions. Finally, as already mentioned before, heterogeneous ice nucleation is a well-accepted formation pathway for ice clouds in the troposphere, suggesting that a similar pathway exists also in the stratosphere.

\section{Table 4: Summary of Section 3}

- There is compelling evidence for widespread heterogeneous nucleation of NAT above $T_{\text {ice }}$ based on spaceborne lidar observations, confirming earlier studies already pointing in this direction.

- Heterogeneous nucleation of NAT on foreign nuclei is slow, leading to low number densities of very large NAT particles. These particles sediment readily and are observed vortex-wide, thus producing efficient denitrification.

- There is some evidence that the heterogeneous nuclei might be of meteoritic origin, though other refractory materials or organics have also been identified.

- Ice-induced NAT nucleation is well-characterized, but requires very low temperatures, which in the Arctic are typically only reached in mountain waves.

- Ice-induced NAT nucleation typically leads to dense clouds of small NAT particles, which move through the vortex and may release individual larger, denitrifying particles.

- As is the case with NAT, there is evidence that ice PSCs may form heterogeneously on foreign nuclei.

- Airborne and spaceborne spectroscopic measurements indicate that NAT particles may be highly aspherical, which can possibly explain heretofore unreconcilable in situ measurements of the largest NAT particles.

\section{Dynamical Forcing of PSCs}

The cooling of the polar winter stratosphere may result in widespread PSC occurrence once synoptic-scale temperatures decrease to that required for their formation. PSCs may form at temperatures below their existence thresholds depending upon the nucleation mechanism and can exist briefly at temperatures above their existence thresholds when evaporating or sublimating.

This article is protected by copyright. All rights reserved. 
Local-scale dynamical processes can also result in an increase in PSC formation and occurrence by enabling small-scale temperatures to fall below their formation threshold values even when the synoptic-scale temperatures remain too high. The PSCs formed by these small-scale dynamical processes may then be advected far downstream of their formation regions.

\subsection{Role of Synoptic Scale Dynamics}

Conditions occur every year in the Antarctic stratospheric winter vortex that are suitable for PSCs, with a relatively small observed range of maximum PSC volume, $\max \left(V_{\mathrm{PSC}}\right) \sim 90-$ $150 \times 10^{6} \mathrm{~km}^{3}$ for the years 2002-2018 (see Figure 12a). By contrast, and as highlighted in Section 2 , in the Arctic no winter is alike and PSC occurrence varies significantly, i.e. $\max \left(V_{\mathrm{PSC}}\right)$ $\approx 5-80 \times 10^{6} \mathrm{~km}^{3}$ (see Figure 12b). Reasons for the differences in cloudiness between the two polar regions are:

(1) The Arctic stratospheric winter vortex generally cools to minimum temperatures well above those found in the Antarctic. This is caused by hemispheric differences in land-sea contrast leading to stronger planetary wave activity in the northern hemisphere (Holton and Hakim, 2013). This enhances the Brewer-Dobson circulation in the northern hemisphere and thus the dynamical compression and heating of air above the Arctic. The resulting temperatures in the Arctic are typically higher than in the Antarctic by around $10 \mathrm{~K}$, and often above PSC formation thresholds as a result.

(2) The Arctic stratospheric polar vortex is typically less concentric than the Antarctic, with wind and temperature fields in the Antarctic normally concentric around the pole - often referred to as "vortex-temperature concentricity" (Lawrence et al., 2015). Polar vortextemperature concentricity is a controlling factor in Arctic denitrification, as the less concentric the vortex the shorter the lifetime of NAT particles, the smaller their size and the lower their denitrification potential (Mann et al., 2002). Synoptic-scale blocking events in the troposphere can induce a strong wave- 1 in the Arctic vortex, with some of these waves having amplitudes as high as $35 \mathrm{~K}$ to which air parcels are exposed while circling the Arctic region (e.g. Peter et al., 1994), suppressing concentricity and NAT persistence.

(3) The Arctic stratospheric winter vortex is more prone than its Antarctic counterpart to localscale temperature perturbations (with horizontal scales from tens to hundreds of kilometers, e.g. by mountain waves), which give rise to localized temperature fluctuations that may help overcome nucleation barriers (Section 4.2).

Figure 31 illustrates Arctic PSC volumes measured by CALIOP and MIPAS, reflecting the high seasonal and inter-annual temperature variability in conjunction with the low concentricity of the Arctic vortex. The pattern in measured PSC volumes in panel (a) closely matches that of the volumes derived from synoptic scale temperatures obtained from ERAInterim reanalysis in panel (b). ERA-Interim reanalysis has a horizontal resolution of T255 (triangular truncation at 255), corresponding to a spatial resolution of approximately $80 \mathrm{~km}$, i.e. resolving synoptic scale dynamics but not mountain waves or other small-scale temperature perturbations. The PSC volumes derived from ERA-Interim by assuming $T<T_{\mathrm{NAT}}$ are highly correlated with the satellite PSC measurements, although the derived volumes are about $50 \%$ too large. The red horizontal bars in Figure $31 \mathrm{~b}$ also show the vortex-wide and season-long timeintegrated volume $V_{\mathrm{PSC}}$ (in units $\mathrm{km}^{3} \times \mathrm{d}$ ) where temperatures are below $T_{\mathrm{NAT}}$, a measure of the potential for ozone depletion related to heterogeneous chemical processing on PSCs (adapted

This article is protected by copyright. All rights reserved. 
from WMO, 2018; following Rex et al., 2002). However, comparison of the measured $V_{\text {PSC }}$ with both the black line and red bars in Figure 31 emphasizes that using $T<T_{\mathrm{NAT}}$ as a constant threshold for chlorine activation in chemical modeling cannot be recommended.

On the other hand, PSC volumes in Figure $31 \mathrm{~b}$ derived from $T<T_{\mathrm{NAT}}-3.5 \mathrm{~K}$ (blue shaded regions) provide an almost perfect match with the measurements in Figure 31a. While NAT clouds can exist at temperatures close to or even slightly higher than $T_{\mathrm{NAT}}$ (until the particles fully sublimate), the reduction in temperature threshold by $3.5 \mathrm{~K}$ can be interpreted as a proxy for including the effects of any neglected microscale and dynamical processes. Examples include STS and ice PSCs occurring at lower temperatures $\left(T_{\mathrm{STS}} \approx T_{\mathrm{NAT}}-3-4 \mathrm{~K}\right.$ and $T_{\text {ice }} \approx T_{\mathrm{NAT}}$ $-7-8 \mathrm{~K}$ ) and local-scale dynamical processes, such as unresolved cooling induced by mountain waves and non-orographic wave activity. These smaller-scale processes are discussed in the subsequent sections.

\subsection{Role of Mesoscale Dynamics}

It has been recognized for some time now that PSCs can be formed in the cool phases of upward-propagating mountain waves, which have horizontal wavelengths on the order of tens to hundreds of kilometers, caused by stratified airflow passing over mountains (Cariolle et al., 1989; Carslaw et al., 1998b), even when synoptic-scale temperatures are above the PSC formation thresholds. Mountain waves generally only persist for a few days at most until the tropospheric weather system that forces the waves moves on (Reichardt et al., 2004). The mountain waves themselves are localized either above the mountain range or in wave trains on the lee side of the range. If the background wind increases with height and is unidirectional, wave refraction results in long vertical wavelengths in the stratosphere that can generate large vertical displacements, resulting in localized adiabatic cooling of up to $\sim 15 \mathrm{~K}$ (e.g. Dörnbrack et al., 1999; 2002). Due to the strong wintertime stratospheric winds, a large amount of air can flow through these small-scale PSCs induced by mountain-wave-cooling, even though they remain stationary above the mountain range, resulting in efficient activation of chlorine (Carslaw et al., 1998a).

As mentioned in Section 3, water ice PSCs formed in the cold phases of temperature fluctuations induced by these mountain waves can seed the formation of so-called NAT "mother" clouds (Fueglistaler et al., 2002a,b). These NAT mother clouds, owing to their 7-8 K higher existence temperature than the triggering ice, are not bound to the cold pools in the mountain waves but may be advected significant distances downstream over time scales of hours to days (Cariolle et al., 1989). Observational evidence of increases in NAT PSCs downstream of ice PSCs formed by temperature perturbations induced by mountain waves are plentiful (e.g. Noel et al., 2009; Lambert et al., 2012; Alexander et al., 2011). An Antarctic case study of a NAT 'belt' observed in mid-June 2003 of PSCs, whose presence was due to large temperature fluctuations associated with mountain waves above the Antarctic Peninsula, showed that the NAT clouds propagated almost entirely around the continent (Höpfner et al., 2006b; Eckermann et al., 2009). The formation of PSCs downwind of the orography due to trapped lee waves can also occur, leading to further PSC production (e.g. Dörnbrack et al., 1999).

Mountain wave forcing of PSCs is an important formation mechanism at the start of the PSC season as well as around the edge of the stratospheric vortex, because synoptic-scale temperatures are above the PSC existence threshold (McDonald et al., 2009; Noel and Pitts, 2012; Lambert et al., 2012). While mountain waves are also observed in mid-winter in both

This article is protected by copyright. All rights reserved. 
hemispheres (Pitts et al., 2013), the air has become so cold at this time that even synoptic-scale temperatures are typically below the PSC existence threshold and the contribution to total PSC occurrence from wave-induced PSCs is small, especially in the Antarctic (Alexander et al., 2013).

The formation of wave-induced PSCs is particularly important in the Arctic (Carslaw et al., 1998a, b; Dörnbrack et al., 2002; Mann et al., 2005). The warmer and more disturbed nature of the Arctic polar vortex results in less frequent synoptic-scale temperatures below the PSC existence threshold than in the Antarctic (Pawson et al., 1995), resulting in wave-induced PSCs having a larger contribution to total PSC occurrence in the Arctic (Dörnbrack \& Leutbecher, 2001; Alexander et al., 2013; Hoffmann et al., 2017). Even during cold Arctic winters, observations indicate that some PSCs were formed in the cool phase of temperature perturbations induced by mountain waves. For example, this was found above Scandinavia, Svalbard and Greenland during the anomalously cold 2009/10 Arctic winter (Pitts et al., 2011; Khosrawi et al., 2011; Dörnbrack et al., 2012; Alexander et al., 2013).

While most studies in the literature have focused on mountain wave sources of PSC production, and meteorological operational and forecast data have become increasingly sophisticated in resolving these waves, there is certainly evidence to indicate that non-orographic gravity wave activity also act as a source of PSCs. Non-orographic waves originating from the upper-tropospheric jet and breaking Rossby waves, with still smaller horizontal wavelengths (less than a few hundred $\mathrm{km}$ ), have been identified as PSC formation sources (Shibata et al., 2003; Hitchman et al., 2003; Buss et al., 2004). Because the 'universal' spectra of atmospheric wind and temperature fluctuations have a negative gradient of power spectral density (Balsley and Carter, 1982), much larger cooling rates occur at large horizontal wavenumber scales (i.e. short wavelengths) than at small horizontal wavenumbers (Murphy and Gary, 1995). The universal nature of the power spectra and the ubiquity of the small-scale wave features indicate that high cooling rates are not restricted to mountain waves (Gary, 2006; 2008). These smallscale fluctuations are important globally in the production of cirrus clouds yet are too small to be resolved in global circulation models (Hoyle et al., 2005). As they affect the number density of cloud ice particles and hence the structure of cirrus clouds and PSCs alike, an improved understanding of small-scale temperature variations remains a task of great importance.

Anticyclonic potential vorticity anomalies in the upper troposphere, which can raise the height of the tropopause, result in synoptic-scale lifting and adiabatic cooling of the stratosphere. This lifting can sufficiently cool the polar winter stratosphere to enable PSCs to exist (Teitelbaum et al., 2001; Fromm et al., 2003) and provides the explanation for ozone 'minihole' events (Teitelbaum et al., 2001; Spang et al., 2001) whereby the elevated tropopause reduces the stratospheric ozone column. These anticyclonic disturbances are likely due to blocking highs in the upper troposphere (Kohma and Sato, 2013), and provide favorable conditions for the simultaneous occurrence of both PSCs and cirrus clouds together (Achtert et al., 2012; Kohma and Sato, 2013).

\subsection{Integrated approach to synoptic and mesoscale dynamical forcing of PSCs}

This section provides a demonstration of an integrated approach to studying PSC formation processes from the multiple perspectives of the MLS, MIPAS, CALIOP, and AIRS (Atmospheric Infrared Sounder) spaceborne instruments. In particular, we examine the development of a circumpolar belt of NAT PSCs, a common phenomenon observed over the

This article is protected by copyright. All rights reserved. 
Antarctic and lasting for several days (e.g., Höpfner et al., 2006b). Localized cooling events associated with mountain waves over the Antarctic Peninsula trigger the homogeneous nucleation of ice and subsequently the heterogeneous nucleation of NAT on ice (Höpfner et al., 2006b; Eckermann et al., 2009). The resulting dense NAT clouds can persist and grow downstream after nucleation through the ice-seeding mechanism (Fueglistaler et al., 2002a; Dhaniyala et al., 2002). The evolution of one such event from 28 May to 2 June 2008 is depicted in Figure 32, as seen through the lens of MLS, MIPAS, CALIOP, and AIRS.

On 28 May 2008, high AIRS brightness temperature variances were detected over the Antarctic Peninsula (Figure 32a) and in addition a gravity wave feature was clearly seen in the GEOS-5 temperature contours over the Peninsula (Lambert et al., 2012). The temperature structure (Figure 32a) provides the potential for ice seeding to form NAT clouds around the circumpolar belt. Over the following six days, MIPAS and CALIOP observed PSCs, including enhanced NAT and patches of ice, appearing downstream in the polar belt in the altitude range $18-26 \mathrm{~km}$, with a typical vertical extent of $4 \mathrm{~km}$ and horizontal extents of several thousand kilometers. Ice PSCs were first detected by MIPAS at altitudes of 20-23 km near the Peninsula (Figure 32b) on 28-29 May and NAT PSCs were detected further downstream on 29 May by both MIPAS and CALIOP. Although missing the earlier MIPAS detections of ice on 28-29 May, CALIOP detected ice PSCs from 30 May-1 June including sporadic wave ice events over the Antarctic Peninsula and the Ellsworth Mountains. From 1 June the PSCs classified by MIPAS as NAT stand out quite markedly against the background of STSmix detections, clustered at the most northerly latitudes and with maximum cloud opacities occurring at greater altitudes. To complete the picture, the response of the distribution of $\mathrm{HNO}_{3}$ is shown by the MLS observations in Figure 32e, where depletion of $\mathrm{HNO}_{3}$ from the gas-phase is associated with synoptic scale STS PSCs close to the pole, and localized depletion of $\mathrm{HNO}_{3}$ is associated with NAT PSCs in the polar belt.

Figure 33 illustrates the potential for ice seeding of NAT clouds and their development downstream from the region of initial ice formation. We calculated forward trajectories on the $500 \mathrm{~K}$ isentropic surface starting from the locations and times that MIPAS ice (Figure 32b and CALIOP wave ice (Figure 32c) were detected over the Antarctic Peninsula and the Ellsworth Mountains. These trajectories enclose the region downstream where the NAT mixture PSCs were detected from 29 May-2 June. On 2 June the northward extent of the NAT mixture PSCs is particularly well bounded by the forward trajectories launched after 30 May.

\subsection{Representation of Orographic Gravity Waves in Models and Reanalyses}

Mesoscale model simulations provide high-resolution trajectory data for microphysical models (Wirth et al., 1999; Voigt et al., 2000). Results from these simulations provided the motivation to apply mesoscale models to predict and analyze PSC observations (e.g. Peter et al., 1992; Volkert and Intes, 1992; Dörnbrack et al., 1998; Cairo et al., 2004; Höpfner et al., 2006b; Eckermann et al., 2006). Figure 34 shows a case study over Scandinavia where PSC backscatter ratio observations, made by an aircraft-borne lidar operating at $1064 \mathrm{~nm}$, are superimposed on mesoscale model output. The PSC backscatter ratio increases markedly downstream of the mountains (indicative of $\mathrm{H}_{2} \mathrm{O}$ ice) and shows clear wave-like oscillations. These correspond closely to the orographic gravity waves generated by the model, indicated in Figure 34 by potential temperature contour lines.

This article is protected by copyright. All rights reserved. 
Weather Research and Forecasting (WRF) modeling studies of wave formation and subsequent PSC formation above the Antarctic Peninsula, along with downstream NAT formation, conducted by Noel and Pitts (2012), compare favorably with CALIPSO PSC observations. Orr et al. (2015) showed that at high horizontal resolution $(4 \mathrm{~km})$, the UK Meteorological Office Unified Model was able to accurately simulate the large mountain-waveinduced temperature fluctuations in the lower stratosphere associated with strong westerly flow over the Antarctic Peninsula. This is illustrated in Figure 35: the simulation captures the amplitude and structure of the mesoscale orographic gravity waves observed by AIRS above the Peninsula.

Efforts have begun on developing and refining parameterizations in global chemistryclimate models that are able to compute the stratospheric temperature fluctuations due to gravity waves (Orr et al., 2015, 2020). These temperature fluctuations would otherwise be missing as current global climate models have a spatial resolution of some hundreds of kilometers and are therefore only able to explicitly resolve waves with relatively long horizontal wavelengths, resulting in insufficient PSC formation and a systematic over-prediction of springtime stratospheric ozone at high-latitudes (Eyring et al., 2006). Efforts are underway to develop variable-resolution global CCMs, such as ICON-ART (ICOsahedral Nonhydrostatic model with Aerosols and Reactive Trace gases; Schröter et al., 2018), which are able to use local grid refinement over mountainous regions in order that the mountain-wave-induced temperature fluctuations are explicitly resolved, obviating the need for their parameterization. Progress towards this goal was limited until detailed observations of PSCs and mountain waves became available, which are essential for a complete evaluation of model results (Höpfner et al., 2006b, Hoffmann et al., 2017; Spang et al., 2018). The temperature fluctuations are also missing from most global atmospheric reanalysis datasets due to their coarse spatial resolution, which are commonly used to force chemistry-transport models (Engel et al., 2013). However, new reanalysis datasets, such as ERA5 and JRA55, have a better spatial resolution of around $50 \mathrm{~km}$, which should be able to better resolve both orographic and non-orographic gravity waves. Indeed, microphysical studies found it necessary to parameterize small-scale non-orographic waves in order for simulations of PSCs to agree with satellite observations (Engel et al., 2013; Tritscher et al., 2019), indicating the importance of these unresolvable waves.

This article is protected by copyright. All rights reserved. 


\section{Table 5: Summary of Section 4}

- Antarctic year-to-year variability of synoptic scale dynamical forcing of PSCs is relatively small, with maximum PSC volumes ranging from $90-150 \times 10^{6} \mathrm{~km}^{3}$.

- In the Arctic, synoptic scale forcing varies dramatically with maximum PSC volumes ranging from $5-80 \times 10^{6} \mathrm{~km}^{3}$, i.e. PSC occurrence varies from negligible to an abundance comparable to that of warmer Antarctic winters.

- Arctic vortex-wide PSC volumes can be approximated by volumes derived from meteorological reanalyses with $T<T_{\mathrm{NAT}}-\Delta T$, where $\Delta T \sim 3.5 \mathrm{~K}$; while this relationship captures synoptic conditions each winter, $\Delta T$ is an empirical quantity, which amalgamates neglected microphysical, mesoscale, and small-scale dynamical processes.

- As has been recognized for some time, PSCs can be formed in the cool phases of mountain (and non-orographic) waves that propagate into the stratosphere, which are mostly unresolved by current global models.

- Mountain wave forcing of PSCs is particularly important where synoptic-scale temperatures are near the PSC existence threshold, such as at the start of the PSC season and close to the edge of the stratospheric vortex.

- Integrated approaches to studying PSC formation, growth, denitrification and dehydration, based on concerted satellite or aircraft instruments combined with microphysical and optical modeling, enable the analysis of PSC formation in mountain waves and their synoptic scale transport downstream in a seamless manner.

- While mesoscale and global atmospheric models, as well as reanalyses, have improved over the last two decades in resolving mountain waves, there is evidence that nonorographic waves also act as a source of PSCs, affecting the number density of nucleated ice particles and hence PSC structure. Increased efforts are therefore required to develop parameterizations that are able to compute the temperature fluctuations induced by unresolved waves, as well as next generation variable-resolution global models that use local grid refinement over mountainous regions.

\section{Heterogeneous Chemistry, Denitrification, and Dehydration by PSCs}

Polar ozone depletion is driven by enhanced levels of $\mathrm{ClO}$ produced by heterogeneous reactions that occur on the surface of PSC particles (Solomon et al., 1986; McElroy et al., 1986a,b). Nearly all of the springtime chemical O ${ }_{3}$ loss occurs through a catalytic cycle initiated by the self-reaction of $\mathrm{ClO}$ (Molina and Molina, 1987) or through other cycles that begin with the reaction of $\mathrm{ClO}$ with $\mathrm{BrO}$ (McElroy et al., 1986a). An overview of the impacts of PSCs during formation of the ozone hole is given in Figure 36, which shows satellite-derived maps of chemical compounds and temperature at $18 \mathrm{~km}$ altitude over Antarctica on 15 September 2008. At this time, temperatures are low, $\mathrm{HCl}$ and $\mathrm{ClONO}_{2}$ have been converted to highly reactive $\mathrm{ClO}$, while $\mathrm{HNO}_{3}$ has been removed by the gravitational settling of PSC particles. The

This article is protected by copyright. All rights reserved. 
abundance of $\mathrm{ClO}$ near the South Pole is low in mid-September due to limited sunlight, which is required to form $\mathrm{ClO}$ from photolysis of $\mathrm{Cl}_{2}$ and $\mathrm{ClOOCl}$. Closer to the edge of the polar vortex, the high values of $\mathrm{ClO}$ cover an extensive area that, at times, exceeds that of the Antarctic continent and can last for several months, leading to efficient destruction of ozone in sunlit regions. Chemical ozone depletion is still ongoing at this time as ozone typically reaches its minimum values in early to mid-October. In the following subsections we review the roles of heterogeneous chemical reactions on PSC surfaces which lead to the activation of chlorine. Further, we describe the importance of gravitational settling of PSC particles that remove $\mathrm{HNO}_{3}$ (denitrification) and $\mathrm{H}_{2} \mathrm{O}$ (dehydration), and highlight the differences between the Arctic and Antarctic polar vortices.

\subsection{Heterogeneous Chemical Reactions on/in PSC Particles}

In the absence of heterogeneous processing, inorganic chlorine $\left(\mathrm{Cl}_{\mathrm{y}}\right)$ in the lower stratosphere of the polar vortices is present almost entirely as $\mathrm{HCl}$ and $\mathrm{ClONO}_{2}$ (Solomon, 1988; Wilmouth et al., 2006), which are termed reservoir species because neither compound is reactive towards $\mathrm{O}_{3}$. Conversely, $\mathrm{BrO}$ constitutes nearly half of the inorganic bromine $\left(\mathrm{Br}_{\mathrm{y}}\right)$ that is present in the lower stratosphere during sunlit conditions (Avallone and Toohey, 2001) under gas phase chemistry. The large difference between $\mathrm{ClO} / \mathrm{Cl}_{\mathrm{y}}(\ll<1)$ and $\mathrm{BrO} / \mathrm{Br}_{\mathrm{y}}(\sim 0.5)$ results mainly from the fact that $\mathrm{Cl}+\mathrm{CH}_{4} \rightarrow \mathrm{HCl}+\mathrm{CH}_{3}$ is exothermic, whereas $\mathrm{Br}+\mathrm{CH}_{4} \rightarrow \mathrm{HBr}+\mathrm{CH}_{3}$ is endothermic, and the resulting molecule $\mathrm{HCl}$ is much more stable than $\mathrm{HBr}$.

Reactions that occur on the surface or in the bulk of PSC particles are central to our understanding of polar ozone depletion. The two main inorganic chlorine reservoirs present during the incipient phase of the winter-time polar vortex, $\mathrm{HCl}$ and $\mathrm{ClONO}_{2}$, do not react in the gas phase in the lower stratosphere. However, heterogeneous reactions such as:

$$
\begin{aligned}
& \mathrm{ClONO}_{2}+\mathrm{HCl}(c) \stackrel{\text { het }}{\longrightarrow} \mathrm{HNO}_{3}(c)+\mathrm{Cl}_{2}, \\
& \mathrm{ClONO}_{2}+\mathrm{H}_{2} \mathrm{O}(c) \stackrel{\text { het }}{\longrightarrow} \mathrm{HNO}_{3}(c)+\mathrm{HOCl}, \\
& \mathrm{HOCl}+\mathrm{HCl}(c) \stackrel{\text { het }}{\longrightarrow} \mathrm{H}_{2} \mathrm{O}(c)+\mathrm{Cl}_{2}, \\
& \mathrm{~N}_{2} \mathrm{O}_{5}+\mathrm{H}_{2} \mathrm{O}(c) \stackrel{\text { het }}{\longrightarrow} \mathrm{HNO}_{3}(c)+\mathrm{HNO}_{3},
\end{aligned}
$$

were postulated to occur on the surface of PSCs, with the Cl-bearing products being photolabile, readily reacting with $\mathrm{O}_{3}$ in catalytic cycles, and the $\mathrm{HNO}_{3}$ and $\mathrm{H}_{2} \mathrm{O}$ remaining in the condensed phase, indicated by (c) (Solomon et al., 1986; McElroy et al., 1986b; Crutzen and Arnold, 1986; Solomon, 1988; Abbatt and Molina, 1992a,b). Reactions R5.1 and R5.2 are responsible for most of the chlorine activation that transforms the chlorine reservoirs $\mathrm{HCl}$ and $\mathrm{ClONO}_{2}$ into active chlorine $\mathrm{ClO}_{\mathrm{x}}$. Reaction $\mathrm{R} 5.3$ also activates chlorine, especially in environments with low $\mathrm{ClONO}_{2}$, while reaction R5.4 is responsible for converting $\mathrm{NO}_{\mathrm{x}}$ to $\mathrm{HNO}_{3}$, especially during polar night in early winter. Early laboratory experiments confirmed that the heterogeneous reactions R5.1 and R5.2 are indeed very effective on ice and on $\mathrm{HNO}_{3} / \mathrm{H}_{2} \mathrm{SO}_{4}$-containing ice surfaces at low temperatures such as those occurring in the wintertime polar stratosphere (Tolbert et al., 1987; Leu, 1988). These studies were still constrained to solid surfaces (ice and NAT-like), as the importance of reactions on/in liquid droplets was unknown at that time. Only later did it become clear that these chemical reactions occur at a similar rate (per unit surface area density,

This article is protected by copyright. All rights reserved. 
SAD) on/in liquid droplets. However, since the SAD of liquid PSCs is usually greater than the SAD of solids, winter chlorine activation is typically dominated by liquid PSCs .

\subsection{Seasonal Evolution of Inorganic Chlorine Species}

We first examine the impact of the fast reactions R5.1-R5.4 on the evolution of inorganic chlorine species under conditions of the cold Antarctic winter stratosphere. Due to the much lower temperatures in the Antarctic compared to the Arctic, there are differences in the occurrence of PSCs between the hemispheres with consequences for ozone depletion. The low temperatures in the Antarctic stratosphere permit ubiquitous PSCs over the winter months (Pitts et al., 2018) and an annual formation of an ozone hole with very few exceptions (Grooß et al., 2005). Temperatures in the Arctic are much more variable from year to year, as is the ozone depletion, which even in years with the coldest and longest vortices only barely reaches the levels seen in the Antarctic.

Once the temperature falls below a critical threshold, about $195 \mathrm{~K}$, gas phase $\mathrm{HCl}$ and $\mathrm{ClONO}_{2}$ are rapidly converted to active chlorine species such as $\mathrm{ClO}, \mathrm{ClOOCl}, \mathrm{HOCl}$, and $\mathrm{Cl}_{2}$, as illustrated schematically in Figure 37. These chlorine activation reactions occur both on the surface and in the bulk of STS and SSA particles, as well as on the surface of NAT and ice particles (Peter, 1997; Solomon et al., 1999; Drdla and Müller, 2012; Grooß et al., 2018).

Reactions R5.1 and R5.2 provide the initial conversion of reservoirs $\mathrm{HCl}$ and $\mathrm{ClONO}_{2}$ to active form in both the Antarctic and Arctic vortices (e.g., Solomon et al., 1986; Salawitch et al., 1988, Crutzen et al., 1992; Grooß et al., 2011; Wohltmann et al., 2017). In particular, R5.1 leads to a titration of $\mathrm{HCl}$ and $\mathrm{ClONO}_{2}$ as the first rapid activation step, in which $\mathrm{ClONO}_{2}$, the less abundant of the two reservoirs, can be consumed entirely, and $\mathrm{HCl}$ decreases at the same time by the same amount (Figure 37a). This first step can happen within hours of the first PSC encounter, e.g. when air is lifted and cooled in mountain waves (Carslaw et al., 1998a). The remaining $\mathrm{HCl}$ after the initial titration can be activated only much more slowly, either via R5.1 after some $\mathrm{ClONO}_{2}$ is reformed through chlorine deactivation, or via $\mathrm{R} 5.3$ with $\mathrm{HOCl}$ produced by $\mathrm{ClO}+$ $\mathrm{HO}_{2} \rightarrow \mathrm{HOCl}+\mathrm{O}_{2}$ (e.g. Crutzen et al., 1992).

Reaction R5.3 plays a much more important role in the Antarctic than the Arctic, due in part to much lower values of $\mathrm{NO}_{\mathrm{x}}$ in the Southern Hemisphere, driven by the lower temperatures, that suppresses reformation of $\mathrm{ClONO}_{2}$ (Crutzen et al., 1992). A recent model analysis indicates about $70 \%$ of the loss of $\mathrm{HCl}$ is driven by Reaction $\mathrm{R} 5.3$ during the chlorine activation phase of the Antarctic, whereas only about $30 \%$ of the loss of $\mathrm{HCl}$ occurs via this route in the Arctic (Wohltmann et al., 2017). Reaction R5.3 also becomes increasingly important for maintaining active chlorine as $\mathrm{O}_{3}$ levels within the Antarctic vortex approach the seasonal minimum (Grooß et al., 2011). Eventually $\mathrm{O}_{3}$ levels within the Antarctic vortex become so low that the most dominant form of active chlorine within the vortex is atomic $\mathrm{Cl}$. At this stage, $\mathrm{HCl}$ is reformed by gas phase reactions such as $\mathrm{Cl}+\mathrm{CH}_{4}$ and $\mathrm{Cl}+\mathrm{H}_{2} \mathrm{CO}$ and levels of active chlorine in the vortex undergo a marked decline. In these cases of very low $\mathrm{O}_{3}$ mixing ratios below about 0.1 ppmv, it is possible that all available chlorine is converted to $\mathrm{HCl}$ and heterogeneous chlorine activation is stopped because of a missing reaction partner even though PSCs may be present (Grooß et al., 2011). Low levels of $\mathrm{NO}_{\mathrm{x}}$ resulting from reaction R5.4 have the consequence that the deactivation of chlorine by the reaction $\mathrm{ClO}+\mathrm{NO}_{2}$ is strongly suppressed, prolonging the period of chlorine-catalyzed ozone depletion (Toon et al., 1986; Crutzen and Arnold, 1986).

This article is protected by copyright. All rights reserved. 


\subsection{Brief Review of Heterogeneous Reaction Probabilities}

The rates of heterogenous reactions R5.1-R5.4 are parameterized in terms of reactive uptake coefficients, also called reaction probabilities $\left(\gamma_{0}\right)$, defined as the number of gas phase reactant molecules colliding with a particle surface and reacting with a molecule on/in the particle divided by the total number of reactant molecules colliding with the surface $\left(\gamma_{0} \leq 1\right)$. Reactions that occur in the bulk of particles (mainly R5.3) are also expressed as surface-based reactions with rates proportional to particle SAD. The reactive uptake coefficient $\gamma_{0}$ is specified as a function of temperature, pressure, and the mixing ratio of gaseous $\mathrm{H}_{2} \mathrm{O}$ based on laboratory observations for SSA, STS, NAT, and $\mathrm{H}_{2} \mathrm{O}$ ice (Hanson et al., 1996; Shi et al., 2001; Hanson, 2003; Burkholder et al., 2015), with the reaction rate (in units of $\mathrm{s}^{-1}$ ) found according to:

$$
k_{\text {het }}=\frac{1}{4} \cdot \gamma_{0} \cdot \mathrm{SAD} \cdot v,
$$

where $\mathrm{SAD}$ is specified per particle composition and $v$ is the mean molecular velocity of the gasphase reactant molecules. The value of $\gamma_{0}$ for reactions R5.1 and R5.3 increases very sharply as a function of decreasing temperature for SSA (Figure 38), resulting in the rapid production of active chlorine and initial titration of gas phase $\mathrm{HCl}$ and $\mathrm{ClONO}_{2}$ once temperature drops below about $195 \mathrm{~K}$ (see chemical lifetimes for a nominal SSA population on the right hand axis of Figure 38).

The main reason for the steep exponential temperature dependence of $\gamma_{0}$ on SSA for reactions R5.1 and R5.3 is the dilution of the aqueous SSA droplets as temperatures drop, allowing $\mathrm{HCl}$ gas to enter the droplets and dissociate to $\mathrm{H}^{+}+\mathrm{Cl}^{-}$. This increase in $\mathrm{HCl}$ solubility was first explained by means of a thermodynamic electrolyte model by Carslaw et al. (1994) (see Figure 27b). Also, $\gamma_{0}$ on SSA for reaction R5.2 increases with decreasing temperature (although not as sharply as $\gamma_{0}$ for R5.1 and R5.3), as the dilution of the SSA droplets with falling temperatures still allows more $\mathrm{H}_{2} \mathrm{O}$ molecules to be freed up to react with $\mathrm{ClONO}_{2}$. The decrease in $\gamma_{0}$ on SSA for R5.2 below $193 \mathrm{~K}$ is due to competition with R5.1, i.e. under such cold conditions the solubility of $\mathrm{HCl}$ becomes sufficiently high and makes R5.1 the dominant reaction pathway. In combination, R5.1 and R5.3 lower the lifetime of $\mathrm{HCl}$ from about 0.5 year to about 1 week when the temperature drops from $200 \mathrm{~K}$ to $195 \mathrm{~K}$.

Figure 39 compares reactions R5.1 and R5.3 on SSA and on STS, NAT, and $\mathrm{H}_{2} \mathrm{O}$ ice PSC particles, but is presented in terms of derived first-order loss rates instead of $\gamma_{0}$ (with nominal chemical lifetimes again shown on the right hand axis). The dashed and solid blue curves in Figure 39a represents the reaction $\mathrm{ClONO}_{2}+\mathrm{HCl}(c)$ on SSA and STS, respectively. The parameterization of this reaction on STS takes into account the uptake of $\mathrm{HNO}_{3}$ into the particles (Figure 27), which increases the droplet surface area by one order of magnitude and explains the approximately tenfold increase in first-order loss rate beyond the one for SSA at temperatures below about $190 \mathrm{~K}$. The first-order loss rate for $\mathrm{ClONO}_{2}+\mathrm{H}_{2} \mathrm{O}(c)$ (Figure 39b) shows a similar increase for STS (solid blue curve) compared to SSA (dashed blue curve) below about $190 \mathrm{~K}$.

The green curves in Figure 39 show the first-order loss rates for reactions R5.1 and R5.2 on NAT particles with $n_{\mathrm{NAT}}=10^{-1} \mathrm{~cm}^{-3}$ (solid curve) and $10^{-4} \mathrm{~cm}^{-3}$ (dashed curve), respectively. The former are representative of the enhanced NAT mixture PSCs primarily observed downwind of wave ice PSCs by CALIOP and MIPAS, while the latter represent the more ubiquitous

This article is protected by copyright. All rights reserved. 
tenuous NAT mixtures observed by both sensors. The loss rates for R5.1 on enhanced NAT mixtures is similar to the loss rates on SSA/STS at temperatures above $193 \mathrm{~K}$. However, CALIOP data (Pitts et al., 2018) show that enhanced NAT PSCs comprise only 4-7\% of total PSCs observed, which effectively reduces their effective loss rate by more than an order of magnitude compared to the ubiquitous liquid droplets. The CALIOP data confirm and generalize earlier ground-based lidar measurements from Ny-Ålesund (Svalbard, Norway), which suggested that only about $1 \%$ of the limited data of three winters were enhanced NAT PSCs, whereas $26 \%$ were tenuous NAT mixtures (Biele et al., 2001).

The cyan curves in Figure 39 show first-order loss rates for reactions R5.1 and R5.3 in wave ice $\left(n_{\text {ice }}=10^{-1} \mathrm{~cm}^{-3}\right.$, solid curve) and synoptic-scale ice $\left(10^{-2} \mathrm{~cm}^{-3}\right.$, dashed curve) PSCs. At first glance, wave-ice-induced $\mathrm{Cl}$ activation appears to be dominant, but CALIOP data (Pitts et al., 2018) show that wave ice PSCs comprise only $0.1 \%$ of all PSCs observed, and in reality most of the activation has already occurred on liquid droplets before widespread ice PSCs are observed.

Another interesting aspect of Figure 39 is that it reveals how different the heterogeneous reaction rates on NAT particles measured by two different laboratories are. The measurements by Abbatt and Molina (1992b) and Hanson and Ravishankara (1993) were made under widely varying conditions, but using a surface reaction model reveals these differences in reactive uptake coefficients by as much as a factor of 100 (Carslaw and Peter, 1997). These differences, though known for a long time, are considered relatively unimportant since heterogeneous chemistry on liquid droplets is under most conditions much more important than that on NAT particles.

Modern models of polar ozone (e.g., Table 1 of Solomon et al., 2015) include the four heterogeneous reactions given above plus at least two additional heterogeneous reactions that involve brominated compounds:

$$
\begin{aligned}
& \mathrm{BrONO}_{2}+\mathrm{H}_{2} \mathrm{O}(c) \stackrel{\text { het }}{\longrightarrow} \mathrm{HOBr}+\mathrm{HNO}_{3}(c), \\
& \mathrm{HOBr}+\mathrm{HCl}(c) \stackrel{\text { het }}{\longrightarrow} \mathrm{BrCl}+\mathrm{H}_{2} \mathrm{O}(c) .
\end{aligned}
$$

There has been a multitude of carefully crafted laboratory measurements combined with physicochemical modeling, which investigated the above-mentioned reactions plus a large number of other potentially important heterogeneous reactions:

$$
\begin{aligned}
& \mathrm{N}_{2} \mathrm{O}_{5}+\mathrm{HCl}(c) \stackrel{\text { het }}{\longrightarrow} \mathrm{ClNO}_{2}+\mathrm{HNO}_{3}(c), \\
& \mathrm{N}_{2} \mathrm{O}_{5}+\mathrm{HBr}(c) \stackrel{\text { het }}{\longrightarrow} \mathrm{BrNO}_{2}+\mathrm{HNO}_{3}(c), \\
& \mathrm{BrONO}_{2}+\mathrm{HCl}(c) \stackrel{\text { het }}{\longrightarrow} \mathrm{BrCl}+\mathrm{HNO}_{3}(c), \\
& \mathrm{ClONO}_{2}+\mathrm{HBr}(c) \stackrel{\text { het }}{\longrightarrow} \mathrm{BrCl}+\mathrm{HNO}_{3}(c), \\
& \mathrm{HOCl}+\mathrm{HBr}(c) \stackrel{\text { het }}{\longrightarrow} \mathrm{BrCl}+\mathrm{H}_{2} \mathrm{O}(c), \\
& \mathrm{HOBr}+\mathrm{HCl}(c) \stackrel{\text { het }}{\longrightarrow} \mathrm{BrCl}+\mathrm{H}_{2} \mathrm{O}(c) . \\
& \mathrm{HOBr}+\mathrm{HBr}(c) \stackrel{\text { het }}{\longrightarrow} \mathrm{Br}_{2}+\mathrm{H}_{2} \mathrm{O}(c) .
\end{aligned}
$$

This article is protected by copyright. All rights reserved. 


$$
\begin{aligned}
& \mathrm{HONO}+\mathrm{HCl}(c) \stackrel{\text { het }}{\longrightarrow} \mathrm{ClNO}+\mathrm{H}_{2} \mathrm{O}(c) . \\
& \mathrm{HONO}+\mathrm{HBr}(c) \stackrel{\text { het }}{\longrightarrow} \mathrm{BrNO}+\mathrm{H}_{2} \mathrm{O}(c) .
\end{aligned}
$$

In each of these reactions, "het" indicates that the reaction requires the surface or bulk of a particle, i.e. either SSA/STS or NAT or ice. Reaction R5.7 provides a minor pathway for conversion of gas phase $\mathrm{HCl}$ to active chlorine. It occurs with a moderate speed on SSA and is therefore also responsible for the conversion of $\mathrm{NO}_{\mathrm{x}}$ to $\mathrm{HNO}_{3}$ in the dark and cold polar vortices in winter resulting in very low $\mathrm{NO}_{\mathrm{x}}$ mixing ratios. Reactions R5.8-R5.13, all of which involve brominated compounds, affect the nighttime reservoir of $\mathrm{BrO}$ and provide a slight enhancement in the rate of $\mathrm{O}_{3}$ loss, due to suppression of daytime $\mathrm{HOBr}$ and $\mathrm{HBr}$ (Lary et al., 1996). However, $\mathrm{BrO}$ is thought to be converted nearly entirely to $\mathrm{BrCl}$ at night when active chlorine is present in the vortex due to a branch of the $\mathrm{ClO}+\mathrm{BrO}$ reaction (Canty et al., 2005; Wohltmann et al., 2017) and the effect of these four reactions on computed polar $\mathrm{O}_{3}$ loss is small, about $2 \%$ (Lary et al., 2006). Similarly, R5.14 and R5.15 have been investigated in a number of laboratory studies (cf. Burkholder et al., 2015), but are likely to play only minor roles under atmospheric conditions. All the laboratory studies have been analyzed and synthesized into recommended values of $\gamma_{0}$ as a function of temperature, pressure, ambient $\mathrm{H}_{2} \mathrm{O}$, etc. by two primary groups: the Subcommittee on Gas Kinetic Data Evaluation for Atmospheric Chemistry of IUPAC (Crowley et al., 2010 and http://iupac.pole-ether.fr) as well as the NASA Panel for Data Evaluation (Burkholder et al., 2019 and https://jpldataeval.jpl.nasa.gov).

\subsection{Reactions on Liquid SSA/STS, NAT, and Ice}

There has been recent attention devoted to assessing the relative importance of reactions occurring on liquid SSA/STS droplets versus reactions on solid NAT particles, for both chlorine activation and $\mathrm{O}_{3}$ loss (Drdla and Müller, 2012; Solomon et al., 2015). As noted earlier, field and laboratory observations both indicate chlorine activation occurs rapidly when the temperature drops below about $195 \mathrm{~K}$ (see Figure 39). Below this temperature, liquid droplets start taking up ambient $\mathrm{HNO}_{3}$ and $\mathrm{H}_{2} \mathrm{O}$ (Carslaw et al., 1994; Peter, 1997). Even though NAT can exist at temperatures below about $195 \mathrm{~K}$ ( $T_{\mathrm{NAT}}$ in the lower stratosphere with nominal levels of $\mathrm{HNO}_{3}$ and $\mathrm{H}_{2} \mathrm{O}$ ), NAT particles are unlikely to nucleate before the air cools to several degrees below $T_{\text {NAT }}$ (Dye et al., 1992). Thus, heterogeneous reactions on liquid aerosols play a significant role for chlorine activation (Solomon et al., 1999). As shown by the dashed and solid blue curves in Figure 39a, much of the initial activation of chlorine takes place on the surface of liquid SSA particles at $T<195 \mathrm{~K}$, where activation rates are shorter than one week, as suggested by Drdla and Müller (2012). A series of sensitivity studies conducted using a global model with specified dynamics concluded "very limited ozone losses occur ... if liquid PSCs or binary aerosols alone are allowed to drive heterogeneous chemistry through reactivities and surface areas characteristic of temperatures of $192 \mathrm{~K}$ or warmer" (Solomon et al., 2015). It is important to note that these simulations also include dehydration, which results in significantly longer chlorine activation rates at $192 \mathrm{~K}$ compared to those displayed in Figure 39. Under dehydrated conditions the reactivity on liquid aerosols above $192 \mathrm{~K}$ is indeed not sufficient to maintain chlorine activation throughout the winter. The simulations of Solomon et al. (2015) also assume $n_{\mathrm{NAT}}=0.01 \mathrm{~cm}^{-3}$ and $n_{\text {ice }}=0.1 \mathrm{~cm}^{-3}$, which except for dense orographically-driven clouds leads to an overestimation of the reactivity on these solid particles. Although $n_{\mathrm{NAT}}$ cannot be derived directly from satellite observations, values between $10^{-4} \mathrm{~cm}^{-3}$ and $10^{-3} \mathrm{~cm}^{-3}$ appear to be more consistent

This article is protected by copyright. All rights reserved. 
with the majority of CALIOP data (Pitts et al., 2018). In situ observations also suggest $n_{\mathrm{NAT}}$ to be in the range of $10^{-4} \mathrm{~cm}^{-3}$ (Voigt et al., 2005) to $10^{-3} \mathrm{~cm}^{-3}$ (Ward et al., 2014). This range of $n_{\text {NAT }}$ is also consistent with simulations of denitrification by sedimenting NAT particles (Carslaw et al., 2002; Grooß et al, 2014).

Despite this debate, the importance of heterogeneous chemistry on the liquid particles (Solomon et al., 1999) remains beyond dispute. For chlorine activation, the first-order loss rates on any existing particle composition would be fast enough to completely deplete $\mathrm{ClONO}_{2}$ in a few days for temperatures at and below $T_{\mathrm{NAT}}$.

\subsection{Denitrification and Dehydration}

Toon et al. (1986) were the first to propose that the sedimentation of PSC particles could play a vitally important role for the formation of the Antarctic ozone hole. The sedimentation of PSC particles containing $\mathrm{HNO}_{3}$ would remove reactive nitrogen (i.e., $\mathrm{NO}_{y}$ ) from the lower stratosphere, preventing the reformation of $\mathrm{NO}$ and $\mathrm{NO}_{2}$ from $\mathrm{HNO}_{3}$ photolysis once the vortex warmed and sunlight returned. Toon et al. (1986) even referred to PSCs as "nitric acid clouds". Model calculations soon demonstrated the importance of denitrification for the development of the ozone hole (e.g., McElroy et al., 1986b; Salawitch et al., 1988; Turco et al., 1989), but were based on relatively crude assumptions. Later studies documented the importance of denitrification for enhancing chemical loss of ozone in the Arctic stratosphere (e.g, Drdla and Schoeberl, 1993; Waibel et al., 1999). The first observational evidence for the sedimentation of stratospheric cloud particles was provided by a depression in the formation temperature of PSCs based on analysis of satellite data (McCormick and Trepte, 1987). Soon after, balloonborne frost point measurements obtained over Antarctica showed that ambient $\mathrm{H}_{2} \mathrm{O}$ could also be removed by the sedimentation of PSC particles (Rosen et al., 1988). Fahey et al. (1989; 1990a) presented aircraft observations from instruments on board the NASA ER-2 aircraft over a series of flights from Punta Arenas, Chile, that revealed the occurrence of both denitrification and dehydration within the Antarctic lower stratospheric vortex. At about the same time, Poole and McCormick (1988) used airborne lidar observations in the Arctic to show that PSC particles form in two distinct stages: one that takes place about 2 to $6 \mathrm{~K}$ above $T_{\text {ice, }}$, now known to involve STS (Carslaw et al., 1994; Tabazadeh et al., 1994) and NAT (Hanson and Mauersberger, 1988); and a second indicating significant ice particle growth below $T_{\text {ice. }}$. Aircraft observations obtained in the Arctic vortex revealed the presence of denitrification in the absence of dehydration (Fahey et al., 1990b). Interestingly, dehydration likely moderates chemical loss of polar ozone because, following the lowering of ambient $\mathrm{H}_{2} \mathrm{O}$, PSC particles subsequently form less readily and the rate of heterogeneous reactions that activate chlorine decline exponentially as a function of available $\mathrm{H}_{2} \mathrm{O}$ (Portmann et al., 1996; Brasseur et al., 1997; Chipperfield and Pyle, 1998). For a proper simulation of the impact of PSCs on polar ozone, models must contain accurate representations of the rate of heterogeneous chemical reactions on the surface of various types of particles, and must properly represent where, when, and how denitrification (and for the Antarctic, dehydration) occurs. The situation is further complicated by the possible presence of NAT particles within clouds that form under low temperature conditions in orographically driven lee waves (Dhaniyala et al., 2002; Fueglistaler et al., 2002a,b; Peter and Grooß, 2012). The resulting clouds with many small NAT particles (enhanced NAT) move downstream and may consequently lead to large, rapidly sedimenting NAT particles, when the largest particles fall out of the lower edge of the cloud (see Subsection 3.2.2.2). These processes are difficult to represent in global models and need to be parameterized (Murphy and Gary, 1995; Gary, 2006; 2008).

This article is protected by copyright. All rights reserved. 
Extensive chemical loss of polar $\mathrm{O}_{3}$ in both hemispheres has always been accompanied by extensive denitrification, i.e. the removal of $\mathrm{NO}_{\mathrm{y}}$ from the polar, lower stratosphere by the sedimentation of PSC particles (see upper right panel in Figure 36). This requires the formation of solid NAT particles (Solomon, 1988; Salawitch et al., 1989; Fahey et al., 2000; Voigt et al., 2000; Manney et al., 2011; Peter and Grooß, 2012; Grooß et al., 2014). As such, NAT particles are considered to be an essential component of our understanding of polar ozone depletion, even though their direct chemical influence via heterogeneous reactions is small compared to SSA/STS (Figure 39). An important condition for effective denitrification is that an optimum number of NAT particles form: neither all pre-existing stratospheric aerosol particles (i.e. about $\left.10 \mathrm{~cm}^{-3}\right)$, as these particles must then stay small $(\sim 0.3 \mu \mathrm{m})$ and sediment only slowly given the limited $\mathrm{HNO}_{3}$ abundance; nor too few particles (e.g. less than $10^{-5} \mathrm{~cm}^{-3}$ ), which would grow and sediment quickly, but remain limited in their denitrification potential. The process of denitrification requires a selective nucleation mechanism forming a small but sufficient number of solid NAT particles (Salawitch et al., 1989; Fahey et al., 2001). As discussed in Section 3, the nuclei achieving this are still under debate and possibly of meteoritic origin.

Finally, when NAT and ice PSC particles sediment into higher temperature regions at lower altitudes, the particles evaporate and produce enhancements in $\mathrm{HNO}_{3}$ (nitrification) and $\mathrm{H}_{2} \mathrm{O}$ (hydration). Simulations have successfully shown to reproduce this effect (Grooß et al., 2005, 2014; Tritscher et al., 2019; see Section 6).

\subsection{Impact of PSCs on Gas Phase Composition}

The rapidity of the loss of $\mathrm{HCl}$ and $\mathrm{ClONO}_{2}$ and the production of $\mathrm{ClO}$ and associated other active chlorine species at temperatures below $~ 195 \mathrm{~K}$ has been confirmed by satellite observations (Santee et al., 1995; von Clarmann et al., 2009; Santee et al., 2011), ground-based and airborne remote observations (Farmer et al., 1987; Solomon et al., 1987a,b; Toon et al., 1989a), as well as in situ measurements (Anderson et al., 1991; Wilmouth et al., 2006) in both hemispheres. Figure 40 shows observations of the ratio of active chlorine with respect to the reservoir gases. While no chlorine activation is seen outside the vortex, inside the cold vortex significant chlorine activation of up to $90 \%$ occurred.

As pointed out above, the seasonal evolution of $\mathrm{Cl}_{\mathrm{y}}$ partitioning shown in Figure 37 is an idealized sketch. Early editions of the WMO/UNEP Ozone Assessment showed a similar sketch as Figure 37 but assuming the mixing ratios of the reservoir gases $\mathrm{HCl}$ and $\mathrm{ClONO}_{2}$ to be very similar at the beginning of the winter and therefore the early titration to be almost complete. Later, this was corrected by Wilmouth et al. (2006, 2018), showing that $\mathrm{HCl}$ stayed finite during the winter, but still reached significantly lower values than suggested by Figure 37 through the longest part of the winter. Interestingly, despite the discovery of the ozone hole dating back more than three decades with the underlying mechanisms believed to be well understood, current stateof-the-art models have problems quantitatively reproducing the observed $\mathrm{HCl}$ in the lower Antarctic stratosphere in early winter (Grooß et al., 2018). While the models describe the development of the ozone hole during the early sunlit period very well, they significantly overestimate $\mathrm{HCl}$, one of the key chemical species, inside the polar vortex during polar night.

The discrepancy exists in different models to varying extent, such as the trajectory-based chemical Lagrangian model CLaMS, as well as in Eulerian models SD-WACCM (the specified dynamics version of the Whole Atmosphere Community Climate Model) and TOMCAT/SLIMCAT. Figure 41 shows some examples, providing a reality check for the

This article is protected by copyright. All rights reserved. 
idealized Figure 37 mentioned above. As argued by Grooß et al. (2018), the $\mathrm{HCl}$ discrepancy hints at some unknown process in the formulation of stratospheric chemistry that is currently not represented in the models. The somewhat smaller discrepancies in the Eulerian models compared to the Lagrangian trajectory model may be traced back to larger numerical diffusion in the transport scheme of the Eulerian models. Although the missing process has not yet been identified, Grooß et al. (2018) investigated a number of hypotheses on the basis of the characteristics of the discrepancy: (i) underestimated $\mathrm{HCl}$ uptake into the PSC particles would show a $T$-dependence different to that observed; (ii) direct photolysis of particulate $\mathrm{HNO}_{3}$ cannot occur in the middle of the winter; (iii) additional production of $\mathrm{NO}_{\mathrm{x}}$ and $\mathrm{HO}_{\mathrm{x}}$ from ionization caused by galactic cosmic rays can explain only about $20 \%$ of the discrepancy; (iv) a hypothetical decomposition mechanism of particulate $\mathrm{HNO}_{3}$, e.g. involving galactic cosmic rays, might resolve the $\mathrm{HCl}$ discrepancy. Although this unknown mechanism is not yet identified, there is some evidence that PSCs may be involved as the difference between observed and simulated $\mathrm{HCl}$ correlates with sunlight time below temperatures of $195 \mathrm{~K}$. It is, however, important to note that the $\mathrm{HCl}$ discrepancy occurs in early winter when a large fraction of the polar vortex is still in darkness, i.e. well before spring when the significant chemical ozone loss occurs. Therefore, there is only a minor impact of about $2 \%$ on the overall ozone column loss over the course of Antarctic winter and spring compared to a simulation with artificially increased chlorine activation.

\section{Table 6: Summary of Section 5}

- Heterogeneous chlorine activation takes place under most conditions on/in liquid SSA/STS droplets, much less on NAT or ice particles.

- Heterogeneous reaction rates on NAT particles measured by different laboratories differ by orders of magnitude, but this is considered relatively unimportant since heterogeneous chemistry is much more important on liquid particles than on NAT.

- Extensive chemical loss of polar $\mathrm{O}_{3}$ in both hemispheres is always accompanied by extensive denitrification, i.e. the removal of $\mathrm{NO}_{\mathrm{y}}$ from the polar lower stratosphere by the sedimentation of PSC particles that are able to grow to large sizes.

- The denitrification observed in both hemispheres provides evidence for a selective NAT nucleation mechanism, resulting in NAT number densities suitable for denitrification. It has been speculated that the nucleation might occur on particles of meteoritic origin.

- The rapidity of the loss of $\mathrm{HCl}$ and $\mathrm{ClONO}_{2}$ and the production of $\mathrm{ClO}$ and associated other active chlorine species at temperatures below $\sim 195 \mathrm{~K}$ has been confirmed by satellite and in situ observations in both hemispheres.

- Models appear to systematically overestimate $\mathrm{HCl}$ inside the vortex in early winter. There is evidence suggesting that PSCs are involved in yet unknown chemical processes yielding $\mathrm{HCl}$ decomposition in the dark winter polar vortex. However, this likely has only a minor impact on the total ozone depletion during a winter/spring season.

This article is protected by copyright. All rights reserved. 


\section{Parameterizations of PSCs in Global Models}

This section provides an overview of how PSCs and their impacts are parameterized in global atmospheric models. Despite their critical role in polar stratospheric chemistry, PSCs are often modeled in a rather simplified manner. The degree of complexity in the model formulations ranges from just switching on chlorine activation reactions as soon as the temperature falls below the threshold $T_{\mathrm{NAT}}$ to sophisticated descriptions of PSC microphysics and chemistry. Typically, global models such as Chemical Transport Models (CTMs), Chemistry-Climate Models (CCMs), or Earth System Models (ESMs) cannot reproduce PSCs in time-consuming detail, but rather aim to include the effects of PSCs on the atmospheric chemical composition, especially related to chlorine activation, denitrification, and polar ozone loss. For the simulation of PSC microphysics and chemistry there are, in principle, three relevant aspects: (1) the formation, maintenance and composition of the PSCs, (2) the heterogeneous reactions on the surface or in the bulk of the PSC particles, and (3) the vertical redistribution of $\mathrm{HNO}_{3}$ and $\mathrm{H}_{2} \mathrm{O}$ due to sedimentation of the larger PSC particles.

\subsection{Microphysical and Chemical Processes}

\subsubsection{Formation of PSCs in Large-Scale Models}

The scientific basis of PSC formation has been introduced in Section 3, with Figure 26 showing the different formation pathways of the individual PSC compositions: the nucleation of ice can occur homogeneously or heterogeneously on pre-existing particles, whereas the nucleation of NAT must occur heterogeneously on solid nuclei. Recent studies have demonstrated the importance of heterogeneous nucleation on foreign nuclei, but not much is known about these nuclei other than they may consist of meteoritic or a variety of other materials (Ebert et al., 2016; Schütze et al., 2017; Schneider et al., 2021). Therefore, the simulation of heterogeneous PSC formation requires certain assumptions to be made. In gravity waves, temperatures may quickly drop well below $T_{\text {ice }}$ reaching the homogeneous nucleation threshold of ice (Koop et al., 2000), leading to homogeneous ice nucleation in a large fraction of the liquid SSA droplets before they can take up significant amounts of $\mathrm{HNO}_{3}$. The formation of wave ice is often not well represented in global models since the extent of the local wave structure and the corresponding cooling rates are typically neither explicitly resolved nor parameterized (see Section 4). Also with respect to other aspects of PSC composition (see Table 1), global models cannot reproduce every detail, but rather aim to reproduce the chemical impact of PSCs as realistically as possible.

The modeling of PSCs requires not only the determination of the composition class (ice, NAT, STS) or mixture of classes (e.g., STS/NAT) at the time of PSC formation, but also their further evolution. For example, NAT and ice particles grow or shrink after a change in temperature until they reach thermodynamic equilibrium by depleting/enriching gas-phase $\mathrm{H}_{2} \mathrm{O}$ and $\mathrm{HNO}_{3}$. Similarly, the liquid SSA droplets take up $\mathrm{HNO}_{3}$ from the gas phase to form STS, enhancing the $\mathrm{HNO}_{3}$ vapor pressure of the $\mathrm{HNO}_{3}-\mathrm{H}_{2} \mathrm{SO}_{4}-\mathrm{H}_{2} \mathrm{O}$ solution and depleting the $\mathrm{HNO}_{3}$ in the gas phase until equilibrium between both is reached. Instead of treating these timedependent kinetic processes in an explicit manner, large-scale models commonly assume thermodynamic equilibrium between the gas phase and PSC particles. For liquid STS, this steady-state assumption is fully justified, since liquid particles of $0.1 \mu \mathrm{m}$ radius need only about 10 min to equilibrate with gas-phase $\mathrm{HNO}_{3}$ (Meilinger et al., 1995). This is not the case for NAT

This article is protected by copyright. All rights reserved. 
particles in low number densities, where it may take days to reach equilibrium (Carslaw et al., 2002).

\subsubsection{Modeling Heterogeneous Chemical Reactions}

The importance of heterogeneous chemistry on SSA and PSC particles of different composition was emphasized in Section 5 with the key reactions R5.1-R5.15, sorted roughly in decreasing order of significance. These reactions can have both surface-based and volume-based components, but are typically parameterized in the models as purely surface-based reactions. Besides heterogeneous chlorine activation in the polar stratosphere, the deactivation of $\mathrm{NO}_{\mathrm{x}}$ and formation of $\mathrm{HNO}_{3}$ via heterogeneous $\mathrm{N}_{2} \mathrm{O}_{5}$ hydrolysis (R5.4) affects the chemical composition of the polar vortex in early winter, making it more prone to strong ozone depletion. While with respect to the heterogeneous reactions on PSCs, there has been no major new research within the last two decades, some contradictory parameterizations of heterogeneous reaction rates on NAT surfaces remain, as shown in Figure 39 (Abbatt and Molina, 1992b; Hanson and Ravishankara, 1993; Wegner et al., 2012). However, this detail is believed to not have a significant impact on model results, since the reactions on liquid SSA and STS are typically faster in this temperature range. Established chemistry modeling schemes include most of the heterogeneous reactions R5.1-R5.15 occurring on any of the PSC particle classes.

\subsubsection{Size- and Shape-Dependent Particle Sedimentation}

PSC particles undergo sedimentation, at a speed that depends mainly on particle size. For spherical particles, the sedimentation speed scales approximately with the square of particle radius and is roughly $1 \mathrm{~m} / \mathrm{h}$ for $1 \mu \mathrm{m}$ radius and $100 \mathrm{~m} / \mathrm{h}$ for $10 \mu \mathrm{m}$ radius. The impact of PSC particle sedimentation is parameterized differently in various models, ranging from fixed vertical velocities for a given PSC class to Lagrangian tracking of individual particles, see Table 7. Besides particle size, the sedimentation velocity depends to lesser degrees on atmospheric pressure (Müller and Peter, 1992) and on particle shape (Woiwode et al., 2014), as non-spherical particles have a larger drag, which has been used to explain certain observations of particularly large PSC particles (Molleker et al., 2014; Woiwode 2016).

An accurate simulation of sedimentation can further be challenging, because very large PSC particles (ice or NAT) may sediment through a significant fraction of a vertical model grid box in a model time step, which may cause problems with numerical diffusion. Large NAT or ice particles with $r>5 \mu \mathrm{m}$, though they may occur only in very low particle number densities on the order of $10^{-3}-10^{-4} \mathrm{~cm}^{-3}$ (Fueglistaler et al., 2002b), are responsible for significant vertical redistribution of $\mathrm{HNO}_{3}$ or $\mathrm{H}_{2} \mathrm{O}$. This sedimentation process leads to denitrification and dehydration and, upon their evaporation, to re-nitrification and re-hydration at lower altitudes. Owing to their importance, these large NAT particles have sometimes been labeled as "NAT rocks" (Fahey et al., 2001; Fueglistaler et al., 2002; Riviere et al., 2003; Molleker et al., 2014).

\subsection{Modeling PSCs and their Impacts}

Depending on the specific modeling purpose, PSCs and their impacts are represented in current atmospheric models in rather different ways. Owing to the complexity of the mechanisms, the treatment in models does not usually take into account the full, detailed PSC characteristics, such as heterogeneous or homogeneous nucleation, PSC composition class, NAT

or ice number density, etc. When the aim of a model study is mainly to describe the impact of the

This article is protected by copyright. All rights reserved. 
PSCs on ozone on the vortex scale, simplifications can be justified, since many processes involving PSCs 'saturate', e.g. after the available chlorine has been fully activated because the chemical reactivity and the available PSC surface area densities increase rapidly below $T_{\mathrm{NAT}}$.

Therefore, a realistic simulation of the outcome of heterogeneous chemical processing does not necessarily require a detailed representation of the processes involved. For example, in the beginning of the polar winter, the heterogeneous reaction $\mathrm{HCl}+\mathrm{ClONO}_{2}(\mathrm{R} 5.1)$ undergoes a titration step until all available $\mathrm{ClONO}_{2}$ has reacted with $\mathrm{HCl}$. The duration of this step may be short compared with the timescales relevant for ozone depletion. There are only rare examples when the initial chlorine activation step does not fully deplete the available $\mathrm{ClONO}_{2}(\mathrm{Nakajima}$ et al., 2016). Hence, the actual reaction rate may not be very relevant due to the steep relationship between ambient temperature and activation time (see Figure 39). Another example of a saturation effect is the maintenance of elevated active chlorine levels in the Antarctic lower stratosphere through $\mathrm{HCl}$ null cycles. Very different assumptions for the rate of reaction $\mathrm{HOCl}+$ $\mathrm{HCl}$ (R5.3) yield similar modeled ozone depletion (Grooß et al., 2011; Müller et al., 2018). In addition, the impact on ozone depletion of omitting some PSC composition classes in models has been studied (Kirner et al., 2015; Solomon et al., 2015). Kirner et al. (2015) showed that omitting the heterogeneous chemistry on NAT particles with $n_{\text {NAT }}$ of about $3 \times 10^{-5} \mathrm{~cm}^{-3}$ has almost no direct impact on chlorine activation (although the indirect effect via denitrification is a strong one). Only NAT-containing clouds with high number densities, i.e. enhanced NAT mixtures with $n_{\mathrm{NAT}}=0.01 \mathrm{~cm}^{-3}$, can be expected to have sufficiently large surface area densities to have a significant local impact on heterogeneous chemistry, and this only when assuming the high heterogeneous reaction probabilities measured by Hanson and Ravishankara (1993) (see green curves in Figure 39). However, only 4-7\% of total PSCs observed by CALIOP are classified as enhanced NAT mixtures (Pitts et al., 2018), so that their vortex-wide impact will remain limited (see Section 5.3). Correspondingly, Solomon et al. (2015) showed in simulations zeroing halogen heterogeneous rates on liquid particles that about half of the ozone loss could be reproduced when assuming dense NAT and ice PSCs $\left(n_{\mathrm{NAT}}=0.01 \mathrm{~cm}^{-3}\right.$ and $\left.n_{\mathrm{ice}}=0.1 \mathrm{~cm}^{-3}\right)$ and the reaction rates measured by Hanson and Ravishankara (1993).

Other processes that may experience saturation are denitrification and dehydration. When temperatures remain below the threshold $T_{\mathrm{NAT}}$ or $T_{\text {ice }}$ for a significant time, denitrification or dehydration may be close to the maximum possible freeze-drying effect, i.e. so much NAT or ice has been removed by gravitational settling that the formation of further NAT or ice becomes increasingly unlikely. If denitrification is overestimated in the early phase of the winter, it would hinder NAT formation and further denitrification later in the season due to less available $\mathrm{HNO}_{3}$, leading to a partial compensation. The largest differences between various parameterizations of denitrification are therefore expected during the onset of the process. However, simplified parameterizations have difficulties in correctly simulating details of $\mathrm{NO}_{\mathrm{y}}$ redistribution and chlorine activation when temperatures are close to the threshold temperature. Also, empirical model parameterizations may not be valid in conditions caused by a changing climate. It is therefore important to test the parameterized PSC schemes in global models against sophisticated explicit models or, when available, validate them against dedicated observations.

Finally, we emphasize that the accuracy of the temperature in the models is very critical. As the processes involving PSCs are strongly temperature-dependent, the PSC parameterizations rely on a very good simulation of stratospheric temperatures. CTMs, which use the temperatures from assimilated data (meteorological analyses or re-analyses), typically have realistic synoptic-

This article is protected by copyright. All rights reserved. 
scale temperatures, but this is often not the case for free-running CCMs or ESMs. These models often suffer from a temperature bias (the so-called "cold bias") in the polar stratosphere that can be on the order of $5 \mathrm{~K}$ or even larger (e.g. Butchart et al., 2011). Such a misrepresentation of polar stratospheric temperatures would cause an exaggerated ozone depletion, regardless of the sophistication of the PSC scheme. Conversely, as discussed in Section 4, CCMs/ESMs poorly represent fine-scale temperature fluctuations induced by orographic and non-orographic gravity waves, which are also important for PSC formation and may partly compensate the cold bias apparent in many of these models.. Section 4 describes efforts to (directly) parameterize the stratospheric temperature fluctuations due to such waves, which otherwise would be missing (Orr et al., 2015; 2020). Alternatively, an (indirect) way is to adjust the number densities of solid particles in an appropriate manner so that agreement with a measured optical signal (e.g.

CALIOP backscatter) is optimized (Tritscher et al., 2019).

\subsection{Specific Treatment of PSCs in Different Models}

For selected global models, this subsection gives an overview of how PSC microphysics and chemistry are parameterized. For comparison, we also discuss process-resolving PSC box models. The Chemistry Climate Model Initiative (CCMI) coordinated simulations of 20 global atmospheric models with the aim of evaluating and comparing results, e.g. regarding the expected recovery of the ozone hole (Dhomse et al., 2018). Most of these models include PSC schemes to calculate chlorine activation. Heterogeneous reactions and PSC compositions implemented in CCMI models are listed in Table S17 and S19 of Morgenstern et al. (2017). However, the focus of these analyses is typically not the simulation of PSCs themselves, but of their chemical impact, in particular on ozone. For this reason, we concentrate in this section on models that specifically report and analyze PSC simulations. Table 7 summarizes this selection of box models and 3-D models and shows details of PSC parameterizations regarding formation, growth and evaporation of the different PSC particle classes, i.e., STS, NAT and ice (whereas other potentially occurring particles, e.g. SAT, and meteoritic dust are typically neglected). Many of the models can also be switched to setups with less sophisticated PSC parameterizations; the table shows the setup with the highest level of complexity. The formation pathway numbers refer to Figure 26. Most models incorporate the heterogeneous reactions R5.1 to R5.13 on all three classes of PSCs.

The representation of PSC formation in these models separates into two groups. In the first group, the models assume thermodynamic equilibrium between particles and the gas phase. This means that during each individual model time step the gas-phase constituents $\mathrm{HNO}_{3}$ and/or $\mathrm{H}_{2} \mathrm{O}$ are assumed to be in thermodynamic equilibrium with the PSC particles as soon as the temperatures fall below the equilibrium temperature, and the gas-phase concentrations are reduced accordingly. In the second group, kinetic effects due to deviations from thermodynamic equilibrium are accounted for. Furthermore, the PSC sedimentation speed is calculated differently, either interactively using the average size of a PSC distribution or, in some models, by a fixed vertical velocity.

This article is protected by copyright. All rights reserved. 
Table 7. Summary of PSC parameterizations in selected box and global models. Note that nearly all models have different options regarding supersaturations required for formation, prescribed number densities, etc. The options listed here are parameterizations from the most sophisticated versions of these models with respect to PSCs. Formation pathways are numbered according to Figure 26. Some models also include SAT particles. Models are listed in alphabetical order within each group.

\begin{tabular}{|c|c|c|c|c|c|c|}
\hline Box Models & Class & Formation mechanism & Pathways & Particle model & Growth & Sedimentation \\
\hline \multirow{3}{*}{$\overline{D M I}$ Model } & STS & diffusive growth & 1 & Eulerian bins & kinetic & no \\
\hline & NAT & surface nucl ${ }^{\mathrm{a}}+$ on SAT & 2(hom), SAT & Eulerian bins & kinetic & no \\
\hline & ice & hom nucl + on NAT & 3 & Eulerian bins & kinetic & no \\
\hline \multirow{3}{*}{ ZOMM } & STS & diffusive growth & 1 & Lagrangian bins & kinetic & no \\
\hline & NAT & het nucl foreign ptcls $/$ ice & 2,6 & Lagrangian bins & kinetic & no \\
\hline & ice & $\begin{array}{l}\text { hom+on foreign+on } \\
\text { NAT }^{\mathrm{c}}\end{array}$ & 4,5 & Lagrangian bins & kinetic & no \\
\hline \\
\hline Lagrangian Models & Class & Formation mechanism & Pathways & Particle model & Growth & Sedimentation \\
\hline \multirow[t]{2}{*}{ ATLAS } & STS & Henry’s Law & 1 & modal $^{\text {n }}$ & equilibrium & no \\
\hline & $\begin{array}{l}\text { NAT } \\
\text { ice }\end{array}$ & $\begin{array}{l}\text { het nucl on foreign } \text { ptcls }^{\mathrm{d}} \\
\text { het nucl on } \mathrm{NAT}^{\mathrm{e}}\end{array}$ & $\begin{array}{l}2 \\
3\end{array}$ & $\begin{array}{l}\text { single particle } \\
\text { modal }\end{array}$ & $\begin{array}{l}\text { kinetic } \\
\text { equilibrium }\end{array}$ & $\begin{array}{l}\text { Lagrangian } \\
\text { no }\end{array}$ \\
\hline \multirow{3}{*}{ CLaMS } & STS & Henry’s Law & 1 & modal $^{\mathrm{n}}$ & equilibrium & \\
\hline & NAT & het nucl foreign ptcls $/$ ice & 2,6 & single particle & kinetic & Lagrangian $^{t}$ \\
\hline & ice & hom+on foreign $\mathrm{ptcls}^{\mathrm{c}}$ & 4,5 & single particle & kinetic & Lagrangian $^{t}$ \\
\hline Eulerian Models & Class & Formation mechanism & Pathways & Particle model & Growth & Sedimentation \\
\hline \multirow[t]{3}{*}{$\overline{B A S C O E}$} & STS & diffusive growth ${ }^{f}$ & 1 & bin model ${ }^{\circ}$ & kinetic & Stokes ${ }^{\mathrm{u}}$ \\
\hline & NAT & surface nucl ${ }^{\mathrm{a}}+$ on SAT & 2(hom), SAT & bin model $^{\circ}$ & kinetic & Stokes $^{\mathrm{u}}$ \\
\hline & ice & hom nucl + on $\mathrm{NAT}^{\mathrm{g}}$ & 3,5 & bin model ${ }^{\circ}$ & kinetic & Stokes $^{\mathrm{u}}$ \\
\hline \multirow[t]{3}{*}{$\overline{E M A C}$ (module PSC) } & STS & Henry's Law & 1 & modal $^{\mathrm{n}}$ & equilibrium & no \\
\hline & NAT & surface nucl $^{\mathrm{a}}+$ on ice & 2 (hom), 6 & bin model ${ }^{p}$ & equil./kinetic & yes \\
\hline & ice & hom+on foreign+on SAT & 4,5, SAT & modal & equil./kinetic & yes \\
\hline \multirow[t]{3}{*}{ LMDz Reprobus } & STS & Henry's Law & 1 & modal $^{\mathrm{n}}$ & equilibrium & no \\
\hline & NAT & het nucl on foreign ptcls & 2 & mean size & equilibrium & ye: \\
\hline & ice & het nucl on NAT & 3 & mean size & equilibrium & yes \\
\hline \multirow[t]{3}{*}{ SOCOL V3.0 } & STS & Henry's Law & 1 & modal $^{\mathrm{n}}$ & equilibrium & \\
\hline & NAT & het nucl on foreign ptcls ${ }^{i}$ & 2 & modal $^{\mathrm{r}}$ & equilibrium & Stokes $^{\mathrm{u}}$ \\
\hline & ice & het nucl on foreign ptcls $\mathrm{s}^{\mathrm{h}, \mathrm{k}}$ & 4 & modal & equilibrium & Stokes $^{u}$ \\
\hline \multirow[t]{3}{*}{ TOMCAT/SLIMCAT } & STS & Henry's Law & 1 & modal $^{\mathrm{n}}$ & equilibrium & \\
\hline & NAT & het nucl on foreign ptcls ${ }^{\mathrm{m}}$ & 2 & single particle & equil./kinetic & Stokes/Lagrangian ${ }^{\mathrm{t}}$ \\
\hline & ice & het nucl on foreign ptcls ${ }^{\mathrm{h}}$ & 4 & modal & equil./kinetic & optional \\
\hline \multirow{3}{*}{$\overline{W A C C M}$} & STS & diffusive growth & 1 & bin model $^{\mathrm{s}}$ & kinetic & Stokes ${ }^{u}$ \\
\hline & NAT & surface nucl ${ }^{\mathrm{a}}+$ on ice & 2 (hom), 6 & bin model $^{\mathrm{s}}$ & kinetic & Stokes $^{\mathrm{u}}$ \\
\hline & ice & hom nucl+on NAT & 3,5 & bin model ${ }^{\mathrm{s}}$ & kinetic & Stokes $^{\mathrm{u}}$ \\
\hline
\end{tabular}

(a) Tabazadeh et al. (2002); (b) Hoyle et al. (2013); (c) Engel et al. (2013); ${ }^{\text {(d) }}$ at supersaturation 5 and prescribed $n_{\mathrm{NAT}}=$ $1 \mathrm{~cm}^{-3}$; ${ }^{(\mathrm{e})}$ at $T_{\text {ice }}$ with prescribed $n_{\text {ice }}=0.01 \mathrm{~cm}^{-3}$; (f) employing PSC module of Larsen et al. (2002; 2004); (g) 3-4 K below $T_{\mathrm{ice}}$; ${ }^{\text {(h) }}$ at $T_{\mathrm{ice}}$; ${ }^{\text {(i) }}$ formed at $T_{\mathrm{NAT}}$; ${ }^{(\mathrm{k})}$ prescribed $n_{\text {ice }}=0.05 \mathrm{~cm}^{-3}$; ${ }^{\text {(m) }}$ constant volume average formation rate $8.1 \times 10^{-10} \mathrm{~cm}^{-3} \mathrm{~s}^{-1}$; ${ }^{(\mathrm{n})}$ Carslaw et al. (1995); ${ }^{\left({ }^{\circ}\right)} 36$ radius size bins from $2 \mathrm{~nm}$ to $36 \mu \mathrm{m}$; (p) 8 radius size bins from 0.1 to $18 \mu \mathrm{m}$; ${ }^{(\mathrm{q})}$ prescribed $n_{\text {ice }}=0.042 \mathrm{~cm}^{-3}$ and $r_{\text {ice }}=0.1 \mu \mathrm{m}$; ${ }^{(\mathrm{r})}$ mean $r_{\mathrm{NAT}}=5 \mu \mathrm{m}, n_{\mathrm{NAT}} \leq 5 \times 10^{-4} \mathrm{~cm}^{-3}$; ${ }^{\text {(s) }} 22$ radius size bins from $0.3 \mathrm{~nm}$ to $20 \mu \mathrm{m}$; ${ }^{(t)}$ Lagrangian simulation of single representative particles (Davies et al., 2005 ); (u) incl. Cunningham slip flow following Pruppacher and Klett (1997).

This article is protected by copyright. All rights reserved. 
Some of the models listed in Table 7 offer specific versions that allow the simulation of PSCs in greater detail than with the standard versions. For example, the Whole Atmosphere Climate Community Model WACCM offers a detailed treatment of PSCs with a parameterization introducing mixtures of NAT and STS (Wegner et al., 2013). This complexity is achieved by allowing only a fraction of the total available $\mathrm{HNO}_{3}$ to condense onto NAT and the remaining part to condense into STS. This scheme uses prescribed size distributions and number densities (e.g., NAT forms at a supersaturation of 10 , about $3 \mathrm{~K}$ below $T_{\mathrm{NAT}}$, at a prescribed particle number density of $10^{-2} \mathrm{~cm}^{-3}$ ). In contrast, Zhu et al. (2015) implemented a very detailed nonequilibrium kinetic PSC module in WACCM, allowing NAT to nucleate in STS particles (Zhu et al., 2017a, b).

There are also model setups in which detailed microphysical PSC models are coupled to a global model. For example, Daerden et al. (2007) coupled the detailed PSC microphysical box model by Larsen et al. (2002) to the Belgian CTM BASCOE (Belgian Assimilation System of Chemical ObsErvations). In the models DLAPSE, which is the Langrangian sedimentation module of TOMCAT/SLIMCAT (Davies et al., 2005), CLaMS (Grooß et al., 2005, 2014; Tritscher et al., 2019), and ATLAS (Alfred Wegener InsTitute LAgrangian Chemistry/Transport System) (Wohltmann et al., 2010), the simulation of PSCs is formulated using a Lagrangian approach such that the simulations follow single representative particles along their trajectories. The Lagrangian setup allows simulation of the vertical redistribution of $\mathrm{NO}_{\mathrm{y}}$ and $\mathrm{H}_{2} \mathrm{O}$ by the sedimentation of NAT and ice particles, respectively. A critical factor in this approach is the parameterization of nucleation processes, which range from a globally constant rate (Davies et al., 2005; Grooß et al., 2005; Wohltmann et al., 2010) to detailed representations of the nucleation efficiency of foreign nuclei in the heterogeneous nucleation process (Grooß et al., 2014; Tritscher et al., 2019).

\subsection{Comparisons of PSC Simulations with Observations}

A comparison of PSC observations with models is not trivial and can involve different levels of complexity. Here, we categorize possible approaches by labels C1 to C6, namely to compare

- C1: geographic volume or area, irrespective of composition class;

- C2: geographic extent for a specific PSC composition class (STS, NAT, ice);

- C3: composition classification along a selected orbit or flight-path curtain;

- C4: PSC size distributions, number densities, or SAD;

- C5: the optical signal of PSCs, e.g. from lidar or limb infrared observations;

- C6: PSC impact on chlorine activation, denitrification, dehydration, and ozone depletion;

- or a combination of the above.

Approaches (C1) and (C2) require the application of PSC detection thresholds to the model output that are comparable to those used by the observational system, e.g., an optical parameter such as the cloud index. A corresponding threshold needs to be defined in the model, e.g., the value or range of simulated PSC SAD in a region that indicates ice, NAT or STS.

Other comparisons with observations include PSC composition classification along a selected orbit or flight-path curtain (C3) or the size distribution and number density of the PSC particles or a parameter like SAD (C4). Mie or T-Matrix calculations allow a comparison of the

This article is protected by copyright. All rights reserved. 
PSC optical signal that, for example, a lidar system or IR limb sounder would observe for the simulated PSCs (C5). Finally, it is possible (C6) to compare the impact of the PSCs on chlorine activation (Wegner et al., 2016) and also the vertical redistribution of $\mathrm{HNO}_{3}$ and $\mathrm{H}_{2} \mathrm{O}$ caused by the PSCs (Grooß et al., 2014; Di Liberto et al., 2015; Tritscher et al., 2019). There are examples of observation-model comparisons for all of these methods.

In the following, we summarize these comparisons with different levels of model complexity ranging from box models to global CCMs, with the focus on global models, in the order given in Table 7. We use the C1-C6 classification above to characterize these comparisons.

Comparisons of the DMI and ZOMM box models with observations. Box models offer the possibility of representing the microphysical and chemical processes with a high level of detail. Two model examples are listed in Table 7. One detailed microphysical box model is the DMI (Danish Meteorological Institute) model ( Larsen et al., 2002). This has been used to investigate PSCs observed in-situ from balloons or remotely by satellites. For instance, for a balloon flight in January 2000, the box model was integrated along mesoscale back trajectories that represented the finer structures of the mountain wave-induced temperature history. A detailed comparison provided insight into PSC formation and growth mechanisms and explained the formation of different PSC compositions along the observed balloon profile (C3). For balloon observations in December 2002, Larsen et al. (2004) demonstrated good agreement between measured and calculated optical properties (C5) assuming the observed PSCs were composed of mixtures of liquid and solid particles. Combining this model with a mountain wave forecast model and an infrared limb radiative transfer scheme, MIPAS observations of an Antarctic NAT belt have been analyzed by comparing measured radiance ratios with simulated ones (Höpfner et al., 2006b) (C5).

Another model is the Zürich Optical and Meteorological Model, ZOMM, which has been employed as box or 1-D column model to simulate PSCs (e.g. Meilinger et al., 1995; Luo et al., 2003; Hoyle et al., 2013; Engel et al., 2013, 2014; Di Liberto et al., 2015) as well as cirrus clouds (e.g. Hoyle et al., 2005; Brabec et al., 2012). ZOMM is initialized at temperatures where the presence of SSA alone can be assumed, with $\mathrm{H}_{2} \mathrm{O}$ mixing ratios from observations, e.g., by MLS. The model is run along individual trajectories, allowing for changing pressure and temperature, where droplets grow and shrink in a full kinetic treatment and without being restricted to the initial lognormal shape of the distribution. Validation of ZOMM results has been performed in different ways (C3-C6).

Comparisons of global models with observations. We discuss below some comparisons of PSC observations with results from the global models listed in Table 7, which includes both Eulerian and Lagrangian CTMs as well as CCMs. Note that most CCMs can be nudged towards reanalyses, resulting in realistic synoptic-scale temperatures - although as discussed above, they would still miss the effects of localized temperature perturbations from mesoscale gravity waves. For the simulation of PSCs, these nudged simulations, often referred to as specified dynamics (SD) mode, are similar to CTM simulations.

Lagrangian CTMs ATLAS and CLaMS. Trajectory-based transport and sedimentation schemes for NAT and ice particles similar to DLAPSE have also been incorporated into the global Lagrangian CTMs CLaMS (McKenna et al., 2002a,b; Konopka et al., 2004; Grooß et al., 2005) and ATLAS (Wohltmann et al., 2010), which emulated most of the concepts of CLaMS, including the Lagrangian simulation of NAT (but not ice) particles. ATLAS was used to simulate

This article is protected by copyright. All rights reserved. 
stratospheric chemistry for the Arctic winter 1999/2000, with a focus on polar ozone depletion and denitrification, validating the model by means of measurements taken during the SOLVE/THESEO 2000 campaign. A model run including detailed treatment of denitrification showed excellent agreement with both long-lived tracers and species from chemical chlorine, nitrogen and hydrogen families, including ozone, measured aboard the ER-2 aircraft and balloons (C6), see Wohltmann et al. (2010).

Grooß et al. (2014) compared PSCs modeled using CLaMS with CALIOP observations on the basis of the optical signal that would be observed by the satellite instrument (C5). The optical signal derived from the simulated PSCs agrees with the observations to within their uncertainty levels, and the vertical redistribution of $\mathrm{NO}_{\mathrm{y}}$ due to sedimenting NAT particles is in accordance with satellite (ACE-FTS, Atmospheric Chemistry Experiment-Fourier Transform Spectrometer) and airborne in situ (SIOUX, Stratospheric Observation Unit for nitrogen oXides) observations (Grooß et al., 2014). A follow-up study byTritscher et al. (2019) provided a comprehensive comparison of the spatial volume of PSCs observed by CALIOP and MIPAS (C1, C2; see Figure 6 of Tritscher et al. 2019), composition classification along CALIOP orbits based on the optical parameters (C3), and the impacts of the particle sedimentation on gas-phase $\mathrm{HNO}_{3}$ and $\mathrm{H}_{2} \mathrm{O}(\mathrm{C} 6)$, reproducing the observed re-hydration of the lower stratosphere resulting from ice PSC particle evaporation. In this study, the detailed structure of PSC composition has also been compared (C3). Figure 42 shows the PSC composition classification by CALIOP along one orbit track on 18 January 2010 and the corresponding results derived from CLaMS (C3). This example demonstrates the ability of the CLaMS model to reproduce the detailed spatial distribution of PSCs by composition, illustrating the robustness of the parameterization of heterogeneous NAT and ice nucleation in CLaMS.

A further example for the comparison of the PSC impact (C6) is a CLaMS simulation of chlorine compounds in early winter 2011 in Antarctica (Grooß et al., 2018). Here, a discrepancy is reported indicating a missing process possibly involving PSCs that is evident also in simulations by other models (see Section 5.6 and Figure 41).

Eulerian CTMs. As discussed above, CTMs use the temperatures from assimilated data, such as meteorological analyses or re-analyses, and thus typically have realistic synoptic-scale temperatures, though mesoscale temperature variations still need to be parameterized.

BASCOE. Incorporating the DMI box model into the global CTM BASCOE allowed Daerden et al. (2007) to produce a detailed 36-bin PSC size distribution, from which extinction coefficients could be derived to compare with satellite observations. Modeled gas phase $\mathrm{HNO}_{3}$ and $\mathrm{H}_{2} \mathrm{O}$ were also compared to satellite (MIPAS and POAM, respectively) observations. Within the model resolution of $1.875^{\circ} \times 2.5^{\circ}$, this approach yielded results that compared well with satellite aerosol extinction data from POAM (C5) and $\mathrm{HNO}_{3}$ from MIPAS (C6).

Reprobus. LMDz Reprobus (Jourdain et al., 2008; Marchand et al., 2012) was the first global model testing a "liquid only" PSC scheme (Lefèvre et al., 1998), which assumed that the particles remain in liquid phase down to $T_{\text {ice, }}$ and used the analytical expression by Carslaw et al. (1995) to calculate the equilibrium composition and volume of the $\mathrm{H}_{2} \mathrm{SO}_{4}-\mathrm{H}_{2} \mathrm{O}$ binary and $\mathrm{HNO}_{3}-\mathrm{H}_{2} \mathrm{SO}_{4}-\mathrm{H}_{2} \mathrm{O}$ ternary droplets as a function of temperature and the total amounts of $\mathrm{H}_{2} \mathrm{O}$, $\mathrm{HNO}_{3}$, and $\mathrm{H}_{2} \mathrm{SO}_{4}$. In this configuration, Reprobus was used to simulate total ozone for the 1996-97 Arctic winter and compared with TOMS satellite measurements (C6). The observed record low ozone values in late March were well reproduced.

This article is protected by copyright. All rights reserved. 
TOMCAT/SLIMCAT. TOMCAT/SLIMCAT (Chipperfield, 2006; Feng et al., 2011) offers two approaches to modeling NAT PSCs, either a modal parameterization with an equilibrium NAT scheme or a Lagrangian approach following individual representative NAT particles called DLAPSE. Feng et al. (2011) used the TOMCAT/SLIMCAT off-line CTM to compare these two approaches in terms of the resulting denitrification (C6). For simulations of the cold Arctic winter 2004/2005 the DLAPSE-based scheme gave a better simulation of the observed denitrification in the Arctic polar vortex than the equilibrium scheme, which overestimated the extent of the denitrification by about $40 \%$ relative to observations (Feng et al., 2011). Analysis of the DLAPSE-based model showed that denitrification caused an additional $30 \%$ ozone loss compared to a run in which it was ignored. The excess denitrification in the equilibrium model produced around 5-10\% more simulated ozone loss. While the DLAPSE code provided a better simulation of denitrification, its implementation in a 3-D model requires a trajectory calculation, which is often prohibitive for the already-expensive CCMs. Parameters in the equilibrium scheme (e.g., particle sedimentation rates) could be adjusted to improve the agreement in any winter, but this level of tuning is not applicable to all possible polar winters.

Free-running climate models: CCMs and ESMs. As mentioned above, these models aim to simulate the past, present and future climate, including that of the polar stratospheres, interacting with the chemistry of the atmosphere. As a drawback, these models may easily have cold biases of a few degrees Kelvin. While this is not a significant problem for simulating the radiative state of the atmosphere or for gas-phase chemistry, such deviations are crucial for PSCs with their sharp formation thresholds. As is the case for CTMs, CCMs and ESMs also typically have too coarse resolution to capture mesoscale temperature fluctuations.

EMAC. In the CCM EMAC (ECHAM5/MESSy Atmospheric Chemistry model), NAT particles are simulated using a bin model, while ice and STS are parameterized using a modal description. The PSC module of EMAC (Kirner et al., 2011) has been used to investigate the impact of different PSC compositions on simulated ozone loss by artificially switching off the presence of individual PSC compositions in the model (Kirner et al., 2015). Observations of $\mathrm{HNO}_{3}, \mathrm{ClO}$ and $\mathrm{O}_{3}$ by MLS have been compared to the model results for validation (C6). Even though the model underestimates polar $\mathrm{HNO}_{3}$ mixing ratios and overestimates polar $\mathrm{O}_{3}$ mixing ratios, the spatial structure and the timing of the $\mathrm{HNO}_{3}$ uptake into the PSC particles are well represented. Khosrawi et al. (2018) compared simulated mean volume densities with volume densities derived from MIPAS observations. While the vertical and temporal distributions are similar, the EMAC simulated volume densities are a factor of three below the lower limit of the observations.

SOCOL. In the SOCOL (modeling tools for studies of SOlar Climate Ozone Links) model (Stenke et al., 2013; Steiner et al., 2021), all PSC compositions are parameterized using a modal setup. Steiner et al. (2021) recently compared CALIOP PSC observations with SOCOLv3 simulations in specified dynamics mode, focusing on Antarctica (C1, C2, C3). The simulated denitrification was evaluated by comparison with $\mathrm{HNO}_{3}$ from MLS (C6). SOCOLv3 considers NAT/STS-mixtures below $T_{\mathrm{NAT}}$ and forms ice below $T_{\text {ice, }}$, with a prescribed ice particle number density, $n_{\text {ice}}$, of $0.01 \mathrm{~cm}^{-3}$. The NAT particle radius $r_{\mathrm{NAT}}$ is fixed at $5 \mu \mathrm{m}$, and the maximum NAT particle number density, $\max \left(n_{\mathrm{NAT}}\right)$, is set to $5 \times 10^{-4} \mathrm{~cm}^{-3}$. The formation of STS via uptake of $\mathrm{HNO}_{3}$ by SSA assumes thermodynamic equilibrium between the condensed phase and the gas phase within each model time-step (following Meilinger et al., 1995). Comparisons with the monthly mean optical signal by CALIOP (depolarization ratio vs. inverse backscatter ratio) were

This article is protected by copyright. All rights reserved. 
made using an optical model (C5), showing a reasonable temporal and spatial agreement of PSC occurrence. However, the coarse model resolution and the fixed ice number density led to an underestimation of mountain-wave-induced ice over Antarctica in the model. For optimized parameters, $\max \left(n_{\mathrm{NAT}}\right)=10^{-3} \mathrm{~cm}^{-3}$ and $n_{\text {ice }}=0.05 \mathrm{~cm}^{-3}$, they found an improved agreement between modeled and observed PSC optical signals (Figures 43a and 43c) and spatial coverage (Figures 43b and 43d), as well as reasonable modeled denitrification. However, the modeled PSC area is overestimated by up to $100 \%$ as a consequence of the cold temperature bias of the model in the polar lower stratosphere, and the fixed $n_{\text {ice }}$ leads to a narrow distribution of ice-containing grid boxes and to a pronounced maximum within the composite histogram.

WACCM. In the WACCM-SD setup of Zhu et al. (2015, 2017a,b), PSCs have been simulated divided into size bins. Good agreement was found between computed STS volume densities and those derived from balloon observations (C4). Furthermore, a detailed comparison was performed with CALIOP PSC composition classes (C3, C5, C6). Figure 44 depicts the PSC occurrence for individual composition classes during the Antarctic winter 2010 simulated by WACCM (Zhu et al., 2017a) and the corresponding quantities derived from CALIOP backscatter measurements (left panels, C1). The center column of Figure 44 shows the areal extent of individual simulated PSC compositions (STS, NAT, ice) without applying the criteria defining the CALIOP composition classes. The right column shows the model results as they would be observed by CALIOP (as STS, MIX and ice). This column is derived from the WACCM results using an optical model (C5). Zhu et al (2017b) showed that the application of the CALIOP category definitions leads to a better comparison of model and observations, including the modeling of NAT particle effective radii of 1-10 $\mu \mathrm{m}$. However, the CALIOP optical signals are still not fully reproduced, especially for large backscatter ratios, and the incorporation of an additional gravity wave scheme did not improve this bias (Zhu et al., 2017b). The simulated dehydration reproduces the observations of MLS, while the denitrification depends strongly on the implied wave amplitude and is overestimated when using the additional gravity wave scheme.

Multi-model comparisons. Snels et al. (2019) compared PSCs from ground-based and satellite lidar observations and compared the derived PSC distributions with results from CCMs employing various methods of including PSCs (see Figure 15). The comparison included four models from the CCMVal-2 project (Eyring et al., 2010) and one model from the CCMI project (Morgenstern et al., 2017). First, Snels et al. investigated the zonal asymmetry of PSC occurrence (C2), for example caused by mountain waves over the Antarctic Peninsula. Second, they compared the derived the SAD for NAT- and ice-containing PSCs (C4). The comparison showed that CCMs suffer from cold biases in the polar stratosphere, which differ among the individual models. In an attempt to validate the PSC schemes in the models independently of this temperature bias, they made a statistical comparison of PSC occurrence as a function of $T-$ $T_{\text {NAT. }}$ However, this comparison still indicated significant differences in the fractional occurrence of PSC composition. For example, the WACCM model, which in general compared best with the observations, still suggests that $95 \%$ of all PSCs in the model at $T_{\mathrm{NAT}}-10 \mathrm{~K}\left(60-82^{\circ} \mathrm{S}\right)$ are ice clouds, whereas CALIOP observations show that the ice fraction amounts to only about $40 \%$.

This article is protected by copyright. All rights reserved. 


\section{Table 8: Summary of Section 6}

- PSCs have been incorporated into various global models with very different degrees of complexity. Typically, simplifications are used that mimic the chlorine activation and the vertical redistribution of $\mathrm{NO}_{y}$ and $\mathrm{H}_{2} \mathrm{O}$. Comparisons of PSC simulations with observations range from PSC geographic distribution and PSC chemical impact to detailed PSC composition and particle sizes.

- A major issue is that free-running CCMs often show significant synoptic-scale temperature biases and, furthermore, miss local temperature fluctuations arising from gravity waves, especially in the polar stratosphere. This results in misrepresentations of PSCs and PSC-related processes due to their strong temperature dependence. However, a mitigating factor is that the impact of PSCs on atmospheric composition is often saturated, i.e. chlorine activation is limited by the available reactants and not by the heterogeneous chemical reaction coefficients, which depend on PSC properties and temperature.

- A detailed PSC scheme (and accurate representation of local temperature fluctuations from gravity waves) is necessary for the simulation of PSCs and their impact in non-saturated cases. This situation occurs, for example, when temperatures are barely low enough for PSC formation, at the beginning/end of the PSC season, or at the geographical edges of activated regions, and hence is more relevant in the Arctic than the Antarctic winter stratosphere.

- Even when compensating for temperature biases, e.g. by nudging to meteorological observations or by scaling temperatures relative to $T_{\mathrm{NAT}}$, models may still show significant discrepancies in PSC composition compared to observations.

\section{Summary and Outlook}

There has been substantial progress in our understanding of PSC spatial and temporal distributions and composition, largely due to the vortex-wide data record in both hemispheres from the spaceborne MIPAS (2002-2012), MLS (2004-present), and CALIOP (2006-present) instruments. For spatially homogeneous cloud scenes, there is general consistency among the three instruments in the major PSC composition classes: STS, liquid-NAT mixtures, and $\mathrm{H}_{2} \mathrm{O}$ ice. CALIOP and MLS data show that STS and ice PSCs occur in near thermodynamic equilibrium, while NAT mixtures show a bimodality depending on exposure time to temperatures below $T_{\text {NAT. }}$. CALIOP and MIPAS multi-year records of PSC coverage are consistent when the MIPAS data are downscaled (by 30-40\% in the Arctic and Antarctic, respectively) to account for PSC patchiness over the large MIPAS FOV. During each winter, PSC composition varies with time, altitude, and spatial position in response to changes in temperature and changes in $\mathrm{HNO}_{3}$ and $\mathrm{H}_{2} \mathrm{O}$ due to denitrification and dehydration. Over decadal timescales, Antarctic PSC coverage is very similar between the CALIOP (2006-2017) and SAM II (1979-1989) eras, whereas there appears to have been a systematic increase in Arctic PSC occurrence in December and January, possibly in response to early winter cooling related to climate change. For the future, measurements of NAT particle shape would lead to more realistic calculations of NAT optical properties and improve the characterization of PSC composition using polarization-sensitive lidars such as CALIOP. Recently, the characteristic spectral signature of large highly aspherical $\beta$-NAT particles has been used to detect populations of large NAT particles vortex-wide in archived MIPAS data from the Arctic winter 2011/12. This method may be used and refined for

This article is protected by copyright. All rights reserved. 
data from future spaceborne infrared instruments.

There have also been significant advances in our understanding of PSC formation processes and particle characteristics. CALIOP observations indicate widespread heterogeneous nucleation of NAT particles at temperatures several $\mathrm{K}$ above $T_{i c e}$, which clearly exceeds the uncertainty of state-of-the-art meteorological analyses. There is evidence that ice PSCs also can form via heterogeneous nucleation. The heterogeneous nuclei may be of meteoritic origin, though other refractory materials or organics have also been identified in stratospheric aerosol particles. Heterogeneous NAT nucleation is slow, leading to low number densities of large NAT particles, an important requirement for efficient denitrification. Ice-induced NAT nucleation, which previously was the only well-characterized mechanism for NAT formation, remains important but requires very low temperatures, which in the Arctic are reached typically only in the cooling phases of mountain waves. Ice-induced NAT nucleation typically leads to dense clouds of small NAT particles ("enhanced NAT mixtures"), which move through the vortex and may release individual larger, denitrifying particles.

Satellite data show that there is relatively little year-to-year variability in synoptic-scale dynamical forcing of PSCs in the Antarctic. In contrast, synoptic-scale dynamical forcing of PSCs in the Arctic varies dramatically from year to year, with PSC abundance ranging from negligible to an amount comparable to that of warmer Antarctic winters. Vortex-wide PSC volumes can be approximated by volumes at $T<T_{\mathrm{NAT}}-3.5 \mathrm{~K}$, as derived from meteorological reanalyses. As has been recognized for some time, PSCs can form in the cool phases of mountain waves that propagate into the stratosphere, which are only partly resolved by current global models. Mountain wave forcing of PSCs is particularly important at the start of the PSC season and near the edge of the stratospheric vortex when/where synoptic-scale temperatures are above PSC formation thresholds (i.e., especially important in the Arctic). While atmospheric models and reanalyses have improved over the last two decades in resolving mountain waves, there is evidence that unresolved non-orographic, small-scale temperature perturbations may also affect the formation of PSCs, affecting the number density of ice particles and hence PSC structure. Increased efforts will be required to develop parameterizations that capture the unresolved temperature fluctuations.

It is now well established that under most conditions, heterogeneous chlorine activation takes place on/in liquid SSA/STS droplets, not on solid NAT or ice particles. Large discrepancies still exist between heterogeneous reaction rates on NAT particles measured by various laboratories, but these are considered relatively unimportant. In addition, extensive chemical loss of polar $\mathrm{O}_{3}$ in both hemispheres appears to require extensive denitrification. The denitrification observed in both hemispheres provides evidence for a selective NAT nucleation mechanism, possibly on nuclei of meteoritic origin, that results in NAT number densities that are optimum for denitrification. An interesting open question is why/how do the cold polar vortices routinely and naturally produce such a seemingly optimum number density of NAT nuclei?

The rapidity of the loss of $\mathrm{HCl}$ and $\mathrm{ClONO}_{2}$ and the production of $\mathrm{ClO}$ and associated other active chlorine species at temperatures below about $195 \mathrm{~K}$ has been confirmed by satellite and in situ observations in both hemispheres. However, models appear to systematically overestimate $\mathrm{HCl}$ inside the vortex, likely due to an unknown process that converts $\mathrm{HCl}$ into active chlorine. This unknown mechanism may involve PSCs since the difference between observed and simulated $\mathrm{HCl}$ correlates with time spent in sunlight and temperatures below 195 $\mathrm{K}$.

This article is protected by copyright. All rights reserved. 
PSCs are incorporated into various global models with a different degrees of complexity. Typically, simplifications are used that mimic chlorine activation and the vertical redistribution of $\mathrm{NO}_{\mathrm{y}}$ and $\mathrm{H}_{2} \mathrm{O}$. Depending on the aim of the simulations, these simplifications may be justified. The detailed reproduction within models of PSCs with respect to particle composition, size distribution, impact on vertical redistribution of $\mathrm{HNO}_{3}$ and $\mathrm{H}_{2} \mathrm{O}$ and impact on chlorine activation and ozone depletion remains challenging. This situation could be improved by further detailed comparisons of model results with the variety of available observations, especially those from satellites. A major issue is that free-running CCMs often show significant temperature biases, especially in the polar stratosphere region which yields misrepresentation of PSCs and PSC-related processes due to their strong temperature dependence. In many cases, the impact of PSCs on atmospheric composition is saturated, especially for very cold stratospheric winters. That means that the chlorine activation is limited by the available reactants and not by the actual heterogeneous chemical reaction speed or denitrification. Furthermore, the concentration of $\mathrm{HNO}_{3}$ or $\mathrm{H}_{2} \mathrm{O}$ available for steady-state uptake into PSC particles in a simple model could be limited by earlier denitrification and/or dehydration, determining what can really sediment out. In these cases, the simulations produce reasonable results despite an incomplete PSC representation in the model. However, there are always non-saturated cases, namely when temperatures are near the PSC formation threshold, or at the beginning/end of the PSC season or at the geographical edges of activated regions. In these cases, a detailed PSC scheme (including, if available, the use of a parameterization to incorporate the effects of temperature fluctuations due to small-scale gravity waves) will yield better results than a simple parameterization. Thus, although there are good arguments for the use of simplified PSC parameterizations in global models, it remains important to understand and simulate the processes in detail, especially when considering PSC effects under climatically changing conditions.

Understanding future changes of stratospheric ozone and climate in the polar regions requires awareness of long-term trends in the global circulation and in the radiative and dynamical heating/cooling of the polar stratosphere. These processes both influence and are influenced by the rate of ozone recovery and by trends in stratospheric GHG abundances, including $\mathrm{H}_{2} \mathrm{O}$, which is still poorly constrained by observations. PSCs, through their formation mechanisms and their role in defining stratospheric composition, can play the twofold role of markers and drivers of climate changes. Therefore, it is important to continue to monitor their long-term trends, for which the development and launch of state-of-the-art spaceborne instruments with capabilities similar to or exceeding those of MIPAS, MLS, and CALIOP is crucial.

\section{Acknowledgments and Data}

This work was inspired by discussions at the SPARC (Stratosphere-troposphere Processes and their Role in Climate)-sponsored workshop on PSCs held at ETH Zurich in August 2014. Out of this workshop, the PSC initiative (PSCi) was established as a SPARC activity in 2015 with the ultimate goal of compiling this review paper. Much of the work was facilitated by a series of meetings of the PSCi team at the International Space Science Institute (ISSI) in Bern, Switzerland. We gratefully acknowledge the support of SPARC and ISSI in this effort. I. Tritscher was funded by the Deutsche Forschungsgemeinschaft (DFG) under project number 310479827. Support for L. Poole is provided under NASA contract NNL11AA10D. M. C. Pitts' work is supported by the NASA CALIPSO-CloudSat Science Team. S. P. Alexander was supported by the Australian Antarctic Science project 4012. A. Lambert's work at the Jet

This article is protected by copyright. All rights reserved. 
Propulsion Laboratory, California Institute of Technology, was carried out under a contract with the National Aeronautics and Space Administration. W. Woiwode was supported by the Karlsruhe House of Young Scientists (KHYS). M. P. Chipperfield was supported by the NERC SISLAC grant (NE/R001782/1) and by a Royal Society Wolfson Merit Award. R. Salawitch appreciates support from the NASA Atmospheric Composition Modeling and Analysis Program and Aura Science Team. We would also like to thank Lars Hoffman (FZJ-JSC) for providing the AIRS gravity wave data and Roland Neuber for providing the $\mathrm{Ny}$-Ålesund lidar data. We are grateful to Ralf Weigel for providing Figure 30; Yunqian Zhu for providing Figure 42; and Michael Steiner for providing Figure 44. The sources of the supporting data are listed in the table below.

\begin{tabular}{|c|c|c|}
\hline Instrument / parameter & Data access & Reference \\
\hline \multicolumn{3}{|l|}{ MIPAS: } \\
\hline $\begin{array}{l}\text { PSC presence and } \\
\text { composition }\end{array}$ & $\begin{array}{l}\text { https://datapub.fz- } \\
\text { juelich.de/slcs/mipas/psc/index.html }\end{array}$ & Spang et al., 2016, 2018 \\
\hline Volume Density & $\begin{array}{l}\text { https://www.imk- } \\
\text { asf.kit.edu/english/308.php }\end{array}$ & Höpfner et al., 2018 \\
\hline & https://doi.org/10.5445/IR/1000086658 & \\
\hline $\begin{array}{l}\text { CALIOP PSC data } \\
\text { products: }\end{array}$ & $\begin{array}{l}\text { https://asdc.larc.nasa.gov/project/CALI } \\
\text { PSO/CAL_LID_L2_PSCMask-Prov- } \\
\text { V1-10_V1-10 }\end{array}$ & Pitts et al., 2018 \\
\hline \multicolumn{3}{|l|}{ MLS: } \\
\hline $\mathrm{H}_{2} \mathrm{O}$ & $\begin{array}{l}\text { https://disc.gsfc.nasa.gov/datasets?page } \\
=1 \& \text { keywords=ML2H2O_004 }\end{array}$ & $\begin{array}{l}\text { Lambert et al (2015), MLS/Aura Level } 2 \\
\text { Water Vapor }\left(\mathrm{H}_{2} \mathrm{O}\right) \text { Mixing Ratio V004, } \\
\text { Greenbelt, MD, USA, Goddard Earth } \\
\text { Sciences Data and Information Services } \\
\text { Center (GES DISC) Accessed: } 1 \text { July } 2020\end{array}$ \\
\hline $\mathrm{HNO}_{3}$ & $\begin{array}{l}\text { https://disc.gsfc.nasa.gov/datasets?page } \\
=1 \& \text { keywords=ML2HNO3_004 }\end{array}$ & $\begin{array}{l}\text { Manney et al (2015), MLS/Aura Level } 2 \\
\text { Nitric Acid (HNO } \text { H Mixing Ratio V004, }_{\text {Greenbelt, MD, USA, Goddard Earth }} \\
\text { Sciences Data and Information Services } \\
\text { Center (GES DISC) Accessed: } 1 \text { July } 2020\end{array}$ \\
\hline $\begin{array}{l}\text { Ground-based Lidar: } \\
\text { McMurdo, Dumont } \\
\text { d'Urville, and Ny-Ålesund }\end{array}$ & http://www.ndaccdemo.org/ & $\begin{array}{l}\text { Snels et al., } 2018 \\
\text { Di Liberto et al., } 2014\end{array}$ \\
\hline
\end{tabular}

This article is protected by copyright. All rights reserved. 


\section{References}

Abbatt, J. P. D., and M. Molina (1992a), The heterogeneous reaction of $\mathrm{HOCl}+\mathrm{HCl} \rightarrow \mathrm{Cl}_{2}+\mathrm{H}_{2} \mathrm{O}$ on ice and nitric-acid trihydrate: Reaction probabilities and stratospheric implications, Geophys. Res. Lett., 19 (5), 461-464, doi: 10.1029/92GL00373.

Abbatt, J. P. D., and M. J. Molina (1992b), Heterogeneous interactions of $\mathrm{ClONO}_{2}$ and $\mathrm{HCl}$ on nitric acid trihydrate at 202 K, J. Phys. Chem., 96, 7674-7679, doi:10.1021/j100198a036.

Achtert, P., and M. Tesche (2014), Assessing lidar-based classification schemes for polar stratospheric clouds based on 16 years of measurements at Esrange, Sweden, J. Geophys. Res. A, 119 (3), 1386-1405, doi:10.1002/2013JD020355.

Achtert, P., M. K. Andersson, F. Khosrawi, and J. Gumbel (2012), On the linkage between tropospheric and Polar Stratospheric clouds in the Arctic as observed by space-borne lidar, Atmos. Chem. Phys., 12, 3791-3798, doi:10.5194/acp-12-3791- 2012.

Adhikari, L., Z. Wang, and D. Liu (2010), Microphysical properties of Antarctic polar stratospheric clouds and their dependence on tropospheric cloud systems, J. Geophys. Res. A, 115 (D4), doi:10.1029/2009JD012125.

Adriani, A., T. Deshler, G. D. Donfrancesco, and G. P. Gobbi (1995), Polar stratospheric clouds and volcanic aerosol during spring 1992 over McMurdo Station, Antarctica: Lidar and

This article is protected by copyright. All rights reserved. 
particle counter comparisons, J. Geophys. Res. A, 100 (D12), 25,877-25,897, doi:10.1029/95JD02029.

Alexander, S. P., A. R. Klekociuk, M. C. Pitts, A. J. McDonald, and A. Arevalo- Torres (2011), The effect of orographic gravity waves on Antarctic polar stratospheric cloud occurrence and composition, J. Geophys. Res., 116, D06109, doi: 10.1029/2010JD015184.

Alexander, S. P., A. R. Klekociuk, A. J. McDonald, and M. C. Pitts (2013), Quantifying the role of orographic gravity waves on polar stratospheric cloud occurrence in the Antarctic and the Arctic, J. Geophys. Res., 118 (20), 11,493-11,507, doi: 10.1002/2013JD020122.

Anderson, J. G., D. W. Toohey, and W. H. Brune (1991), Free radicals within the Antarctic vortex: The role of CFCs in Antarctic ozone loss, Science, 251, 39-46, doi: 10.1126/science.251.4989.39.

Arctowski, H. (1902), Nuages lumineux et nuages irisés, Ciel et Terre, 22, 17-21.

Assmann, R. (1902), Über die Existenz eines wärmeren Luftstromes in der Höhe von 10 bis 15 km., Sitzber. Königl. Preuss. Akad. Wiss. Berlin, 24, 495-504.

Avallone, L. M., and D. W. Toohey (2001), Test of halogen photochemistry using in situ measurements of $\mathrm{ClO}$ and $\mathrm{BrO}$ in the lower polar stratosphere, J. Geophys. Res., 106, 10,411-10,421, doi: 10.1029/2000JD900831.

Balsley, B. B., and D. A. Carter (1982), The spectrum of atmospheric velocity fluctuations at 8 and 86 km, Geophys. Res. Lett., 9, 465-468, https://doi.org/10.1029/GL009i004p00465.

Barone, S. B., M. A. Zondlo, and M. A. Tolbert (1997), A kinetic and product study of the hydrolysis of $\mathrm{ClONO}_{2}$ on Type Ia polar stratospheric cloud materials at $185 \mathrm{~K}, J$. Phys. Chem., 101 (46), 8643-8652, doi:10.1021/jp971107k.

Beyerle, G., H. Deckelmann, R. Neuber, J. M. Rosen, E. Reimer, and M. R. Schoeberl (2001), Occurrence of solid particles in the winter polar stratosphere above the nitric acid trihydrate coexistence temperature inferred from ground-based polarization lidar observations at Ny-Ålesund, Spitsbergen, J. Geophys. Res., 106 (D3), 2979-2992, doi:10.1029/2000JD900569.

Bi, L., P. Yang, G. W. Kattawar, and M. I. Mishchenko (2013), Efficient implementation of the invariant imbedding T-matrix method and the separation of variables method applied to

This article is protected by copyright. All rights reserved. 
large nonspherical inhomogeneous particles, J. Quant. Spectr. Radiat. Transfer, 116, 169-183, doi:https://doi.org/10.1016/j.jqsrt.2012.11.014.

Biele, J., A. Tsias, B. P. Luo, K. S. Carslaw, R. Neuber, G. Beyerle, and T. Peter (2001), Nonequilibrium coexistence of solid and liquid particles in Arctic stratospheric clouds, $J$. Geophys. Res., 106 (D19), 22,991-23,007, doi: 10.1029/2001JD900188.

Biermann, U. M., T. Presper, T. Koop, J. Mossinger, P. J. Crutzen, and T. Peter (1996), The unsuitability of meteoritic and other nuclei for polar stratospheric cloud freezing, Geophys. Res. Lett., 23 (13), 1693-1696, doi:10.1029/96GL01577.

Biermann, U. M., J. N. Crowley, T. Huthwelker, G. K. Moortgat, P. J. Crutzen, and T. Peter (1998), FTIR studies on lifetime prolongation of stratospheric ice particles due to NAT coating, Geophys. Res. Lett., 25 (21), 3939-3942, doi: 10.1029/1998GL900040.

Bogdan, A., and M. Kulmala (1999), Aerosol silica as a possible candidate for the heterogeneous formation of nitric acid hydrates in the stratosphere, Geophys. Res. Lett., 26, 1433-1436, doi: 10.1029/1999GL900254.

Bogdan, A., M. J. Molina, M. Kulmala, A. R. MacKenzie, and A. Laaksonen (2003), Study of finely divided aqueous systems as an aid to understanding the formation mechanism of polar stratospheric clouds: Case of $\mathrm{HNO}_{3} / \mathrm{H}_{2} \mathrm{O}$ and $\mathrm{H}_{2} \mathrm{SO}_{4} / \mathrm{H}_{2} \mathrm{O}$ systems, J. Geophys. Res., 108 (D10), 4302, doi:10.1029/2002JD002605.Bogdan, A., M. J. Molina, H. Tenhu, E. Mayer, and T. Loerting (2010), Formation of mixed-phase particles during the freezing of polar stratospheric ice clouds, Nature Chem., 2, 197-201.

Brabec, M., F. G. Wienhold, B. P. Luo, H. Vömel, F. Immler, P. Steiner, E. Hausammann, U. Weers, and T. Peter (2012), Particle backscatter and relative humidity measured across cirrus clouds and comparison with microphysical cirrus modelling, Atmos. Chem. Phys., 12 (19), 9135-9148, doi:10.5194/acp-12-9135-2012.

Brasseur, G. P., X. Tie, J. Rash, and F. Lefevre (1997), A three-dimensional simulation of the antarctic ozone hole: Impact of anthropogenic chlorine on the lower stratosphere and upper troposphere, J. Geophys. Res., 102, 8909-8930, doi: 10.1029/96JD03398.

Brooks, S. D., D. Baumgardner, B. Gandrud, J. E. Dye, M. J. Northway, D. W. Fahey, T. P. Bui, O. B. Toon, and M. A. Tolbert (2003), Measurements of large stratospheric particles in the Arctic polar vortex, J. Geophys. Res., 108, 4652, doi:10.1029/2002JD003278.

Browell, E. V., C. F. Butler, M. A. Fenn, W. B. Grant, S. Ismail, M. Schoeberl, O. B. Toon, M. Loewenstein, and J. R. Podolske (1993), Ozone and aerosol changes during the 19911992 Airborne Arctic Stratospheric Expedition, Science, 261, 1155-1158, doi: 10.1126/science.261.5125.1155.

Burkholder, J. B., S. P. Sander, J. Abbatt, J. R. Barker, C. Cappa, J. D. Crounse, T. S. Dibble, R. E. Huie, C. E. Kolb, M. J. Kurylo, V. L. Orkin, C. J. Percival, D. M. Wilmouth, and P. H. Wine (2019), Chemical kinetics and photochemical data for use in atmospheric studies,

This article is protected by copyright. All rights reserved. 
evaluation number 19, JPL Publication 19-5, Jet Propulsion Laboratory, Pasadena, http://jpldataeval.jpl.nasa.gov.

Buss, S., A. Hertzog, C. Hostettler, T. P. Bui, D. Lüthi, and H. Wernli (2004), Analysis of jet stream induced gravity wave associated with an observed stratospheric ice cloud over Greenland, Atmos. Chem. Phys., 4, 1183-1200, doi:10.5194/acp-4- 1183-2004.

Butchart, N., A. J. Charlton-Perez, I. Cionni, S. C. Hardiman, P. H. Haynes, K. Krueger, P. J. Kushner, P. A. Newman, S. M. Osprey, J. Perlwitz, M. Sigmond, L. Wang, H. Akiyoshi, J. Austin, S. Bekki, A. Baumgaertner, P. Braesicke, C. Bruehl, M. Chipperfield, M. Dameris, S. Dhomse, V. Eyring, R. Garcia, H. Garny, P. Joeckel, J.-F. Lamarque, M. Marchand, M. Michou, O. Morgen- stern, T. Nakamura, S. Pawson, D. Plummer, J. Pyle, E. Rozanov, J. Scinocca, T. G. Shepherd, K. Shibata, D. Smale, H. Teyssedre, W. Tian, D. Waugh, and Y. Yamashita (2011), Multimodel climate and variability of the stratosphere, J. Geophys. Res. A, 116, doi:10.1029/2010JD014995.

Cairo, F., et al. (2004), Polar stratospheric clouds observed during the Airborne Polar Experiment-Geophysica Aircraft in Antarctica (APE-GAIA) campaign, J. Geophys. Res., 109, D07204, doi:10.1029/2003JD003930.

Campbell, P., and T. Deshler (2014), Condensation nuclei measurements in the midlatitude (1982-2012) andAntarctic (1986-2010) stratosphere between 20 and 35 km, J. Geophys. Res. Atmos., 119, doi:10.1002/2013JD019710.

Canty, T., E. Rivireand, R. Salawitch, G. Berthet, J.-B. Renard, K. Pfeilsticker, M. Dorf, A. Butz, H. B. R. Stimpf, D. Wilmouth, E. Richard, D. Fahey, P. Popp, M. Schoeberl, L. Lait, and T. Bui (2005), Nighttime OClO in the winter arctic vortex, J. Geophys. Res., 110, doi:10.1029/2004JD005035.

Cariolle, D., S. Muller, and F. Cayla (1989), Mountain waves, polar stratospheric clouds and the ozone depletion over Antarctica, J. Geophys. Res., 94 (D9), 11,233-11,240, doi: 10.1029/JD094iD09p11233.

Carslaw, K. S. and T. Peter (1997), Uncertainties in reactive uptake coefficients for solid stratospheric particles-I. Surface chemistry, Geophys. Res. Lett., 24, No. 14, 1743-1746, doi: 10.1029/97GL01683.

Carslaw, K. S., B. P. Luo, S. L. Clegg, T. Peter, P. Brimblecombe, and P. J. Crutzen (1994), Stratospheric aerosol growth and $\mathrm{HNO}_{3}$ gas phase depletion from coupled $\mathrm{HNO}_{3}$ and water uptake by liquid particles, Geophys. Res. Lett., 21 (23), 2479-2482, doi:10.1029/94GL02799.

Carslaw, K. S., S. L. Clegg, and P. Brimblecombe (1995), A thermodynamic model of the system $\mathrm{HCl}-\mathrm{HNO}-\mathrm{H}_{2} \mathrm{SO}_{4}-\mathrm{H}_{2} \mathrm{O}$, including solubilities of $\mathrm{HBr}$, from $328 \mathrm{~K}$ to $<200 \mathrm{~K}, \mathrm{~J}$. Phys. Chem., 99, 11,557-11,574, doi: 10.1021/j100029a039.

Carslaw, K. S., T. Peter, and S. L. Clegg (1997), Modeling the composition of liquid stratospheric aerosols, Rev. Geophys., 35, 125-154, doi: 10.1029/97RG00078.

Carslaw, K. S., M. Wirth, A. Tsias, B. P. Luo, A. Dörnbrack, M. Leutbecher, H. Volkert, W. Renger, J. T. Bacmeister, E. Reimer, and T. Peter (1998a), Increased stratospheric ozone

This article is protected by copyright. All rights reserved. 
depletion due to mountain-induced atmospheric waves, Nature, 391, 675-678, doi: $10.1038 / 35589$.

Carslaw, K. S., M. Wirth, A. Tsias, B. P. Luo, A. Dörnbrack, M. Leutbecher, H. Volkert, W. Renger, J. T. Bacmeister, and T. Peter (1998b), Particle microphysics and chemistry in remotely observed mountain polar stratospheric clouds, J. Geophys. Res., 103, 57855796, doi: 10.1029/97JD03626.

Carslaw, K.S., T. Peter, J. T. Bacmeister, and S. D. Eckermann (1999), Widespread solid particle formation by mountain waves in the Arctic stratosphere, J. Geophys. Res., 104, $1827-$ 1836, doi: 10.1029/1998JD100033.

Carslaw, K. S., J. A. Kettleborough, M. J. Northway, S. Davies, R.-S. Gao, D. W. Fahey, D. G. Baumgardner, M. P. Chipperfield, and A. Kleinböhl (2002), A vortex-scale simulation of the growth and sedimentation of large nitric acid hydrate particles, J. Geophys. Res., 107(D20), 8300, doi:10.1029/2001JD000467.

Chipperfield, M. P. (2006), New version of the TOMCAT/SLIMCAT off-line chemical transport model: Intercomparison of stratospheric tracer experiments, Q. J. R. Meteorol. Soc., 132 (617, B), doi:10.1256/qj.05.51.

Chipperfield, M. P., and J. A. Pyle (1998), Model sensitivity studies of Arctic ozone depletion, J. Geophys. Res., 103, 28,389-28,403, doi: 10.1029/98JD01960.

Córdoba-Jabonero, C., J. L. Guerrero-Rascado, D. Toledo, M. Parrondo, M. Yela, M. Gil, and H. A. Ochoa (2013), Depolarization ratio of polar stratospheric clouds in coastal Antarctica: comparison analysis between ground-based Micro Pulse Lidar and space-borne CALIOP observations, Atmos. Meas. Tech., 6 (3), 703-717, doi:10.5194/amt-6-703-2013.

Crowley, J. N., M. Ammann, R. A. Cox, R. G. Hynes, M. E. Jenkin, A. Mellouki, M. J. Rossi, J. Troc, and T. J. Wallington (2010), Evaluated kinetic and photochemical data for atmospheric chemistry: Volume V - heterogeneous reactions on solid substrates (Vol 10, pg 9059, 2010), Atmos. Chem. Phys., 13 (15), 7359, doi:10.5194/acp-13-7359-2013.

Crutzen, P. J., and F. Arnold (1986), Nitric acid cloud formation in the cold Antarctic stratosphere: A major cause for the springtime 'ozone hole', Nature, 342, 651-655, doi:10.1038/324651a0.

Crutzen, P. J., R. Müller, C. Brühl, and T. Peter (1992), On the potential importance of the gas phase reaction $\mathrm{CH}_{3} \mathrm{O}_{2}+\mathrm{ClO} \rightarrow \mathrm{ClOO}+\mathrm{CH}_{3} \mathrm{O}$ and the heterogeneous reaction $\mathrm{HOCl}+$ $\mathrm{HCl} \rightarrow \mathrm{H}_{2} \mathrm{O}+\mathrm{Cl}_{2}$ in "ozone hole" chemistry, Geophys. Res. Lett., 19 (11), 1113-1116, doi:10.1029/92GL01172.

Curtius, J., R. Weigel, H. J. Vössing, H. Wernli, A. Werner, C. M. Volk, P. Konopka, M. Krebsbach, C. Schiller, A. Roiger, H. Schlager, V. Dreiling, and S. Borrmann (2005), Observations of meteoric material and implications for aerosol nucleation in the winter

This article is protected by copyright. All rights reserved. 
Arctic lower stratosphere derived from in situ particle measurements, Atmos. Chem. Phys., 5, 3053-3069, doi: 10.5194/acp-5-3053-2005.

Cziczo, D. J., D. S. Thomson, and D. M. Murphy (2001), Ablation, flux, and atmospheric implications of meteors inferred from stratospheric aerosol, Science, 291 (5509), 17721775, doi:10.1126/science.1057737.

Daerden, F., N. Larsen, S. Chabrillat, Q. Errera, S. Bonjean, D. Fonteyn, K. Hoppel, and M. Fromm (2007), A 3D-CTM with detailed online PSC-microphysics: analysis of the Antarctic winter 2003 by comparison with satellite observations, Atmos. Chem. Phys., 7, 1755-1772, doi: 10.5194/acp-7-1755-2007.

David, C., P. Keckhut, A. Armetta, J. Jumelet, M. Snels, M. Marchand, and S. Bekki (2010), Radiosonde stratospheric temperatures at Dumont d'Urville (Antarctica): trends and link with polar stratospheric clouds, Atmos. Chem. Phys., 10 (8), 3813-3825, doi:10.5194/acp-10-3813-2010.

Davies, S., G. W. Mann, K. S. Carslaw, M. P. Chipperfield, J. A. Kettleborough, M. L. Santee, H. Oelhaf, G. Wetzel, Y. Sasano, and T. Sugita (2005), 3-D microphysical model studies of Arctic denitrification: comparison with observations, Atmos. Chem. Phys., 5 (11), 3093-3109, doi: 10.5194/acp-5-3093-2005.

Deshler, T. (1994), In situ measurements of Pinatubo aerosol over Kiruna on four days between 18 January and 13 February 1992, Geophys. Res. Lett., 21 (13), 1323-1326, doi:10.1029/93GL03227.

Dhaniyala, S., K. A. McKinney, and P. O. Wennberg (2002), Lee-wave clouds and denitrification of the polar stratosphere, Geophys. Res. Lett., 29 (9), doi: 10.1029/2001GL013900.

Dhomse, S. S., D. Kinnison, M. P. Chipperfield, R. J. Salawitch, I. Cionni, M. I. Hegglin, N. L. Abraham, H. Akiyoshi, A. T. Archibald, E. M. Bednarz, S. Bekki, P. Braesicke, N. Butchart, M. Dameris, M. Deushi, S. Frith, S. C. Hardiman, B. Hassler, L. W. Horowitz, R.-M. Hu, P. Jöckel, B. Josse, O. Kirner, S. Kremser, U. Langematz, J. Lewis, M. Marchand, M. Lin, E. Mancini, V. Marécal, M. Michou, O. Morgenstern, F. M. O’Connor, L. Oman, G. Pitari, D. A. Plummer, J. A. Pyle, L. E. Revell, E. Rozanov, R. Schofield, A. Stenke, K. Stone, K. Sudo, S. Tilmes, D. Visioni, Y. Yamashita, and G. Zeng (2018), Estimates of ozone return dates from chemistry-climate model initiative simulations, Atmos. Chem. Phys., 18, 8409-8438, doi:10.5194/acp-18-8409-2018.

Dieterichs, H. (1950), Mother-of-pearl clouds and their problems, Geofisica Pura e Applicata, 16, 128-132, doi:https://doi.org/10.1007/BF02026314.

DiLiberto, L., F. Cairo, F. Fierli, G. DiDonfrancesco, M. Viterbini, T. Deshler, and M. Snels (2014), Observation of polar stratospheric clouds over McMurdo $(77.85 \circ \mathrm{S}, 166.67 \circ \mathrm{E})$ (2006-2010), J. Geophys. Res. A, 119 (9), 5528-5541, doi: 10.1002/2013JD019892.

Dörnbrack, A., M. Leutbecher, R. Kivi, and E. Kyrö (1999), Mountain-wave-induced record low stratospheric temperatures above northern Scandinavia, Tellus, 51A, 951-963, doi:10.1034/j.1600-0870.1999.00028.x.

Dörnbrack, A., M. Leutbecher, J. Reichardt, A. Behrendt, K.-P. Müller, and G. Baumgarten (2001), Relevance of mountain wave cooling for the formation of polar stratospheric

This article is protected by copyright. All rights reserved. 
clouds over Scandinavia: Mesoscale dynamics and observations for January 1997, $J$. Geophys. Res., 106 (D2), 1569-1581, doi: 10.1029/2000JD900194.

Dörnbrack, A., T. Birner, A. Fix, H. Flentje, A. Meister, H. Schmid, E. V. Browell, and M. J. Mahoney (2002), Evidence for inertia gravity waves forming polar stratospheric clouds over Scandinavia, J. Geophys. Res., 107 (D20), 8287, doi: 10.1029/2001JD000452.

Dörnbrack, A., M. C. Pitts, L. R. Poole, Y. J. Orsolini, K. Nishii, and H. Nakamura (2012), The 2009 - 2010 Arctic stratospheric winter - general evolution, mountain waves and predictability of an operational weather forecast model, Atmos. Chem. Phys., 12, 36593675, doi: 10.5194/acp-12-3659-2012.

Drdla, K., and M. R. Schoeberl (2002), Microphysical modeling of the 1999-2000 Arctic winter 2. Chlorine activation and ozone depletion, J. Geophys. Res., 107, 8319, doi:10.1029/2001JD001159, [printed 108 (D5), 2003].

Drdla, K., and R. Müller (2012), Temperature thresholds for chlorine activation and ozone loss in the polar stratosphere, Ann. Geophys., 30, 1055-1073, doi: 10.5194/angeo-30-1-2012.

Drdla, K., R. P. Turco, and S. Elliott (1993), Heterogeneous chemistry on Antarctic polar stratospheric clouds: A microphysical estimate of the extent of chemical processing, $J$. Geophys. Res., 98( D5), 8965- 8981, doi:10.1029/93JD00164.

Drdla, K., M. R. Schoeberl, and E. V. Browell (2002), Microphysical modeling of the 1999-2000 Arctic winter 1. Polar stratospheric clouds, denitrification, and dehydration, J. Geophys. Res., 107, 8312, doi:10.1029/2001JD000782, [printed 108 (D5), 2003].

Dye, J. E., D. Baumgardner, B. W. Gandrud, S. R. Kawa, K. K. Kelly, M. Loewenstein, G. V. Ferry, K. R. Chan, and B. L. Gary (1992), Particle size distributions in Arctic polar stratospheric clouds, growth and freezing of sulfuric acid droplets and implications for cloud formation, J. Geophys. Res., 97, 8015-8034, doi: 10.1029/91JD02740.

Ebert, M., R. Weigel, K. Kandler, G. Günther, S. Molleker, J.-U. Grooß, B. Vogel, S. Weinbruch, and S. Borrmann (2016), Chemical analysis of refractory stratospheric aerosol particles collected within the Arctic vortex and inside polar stratospheric clouds, Atmos. Chem. Phys., 16 (13), 8405-8421, doi:10.5194/acp-16-8405- 2016.

Eckermann, S. D., D. L. Wu, J. D. Doyle, J. F. Burris, T. J. McGee, C. A. Hostetler, L. Coy, B. N. Lawrence, A. Stephens, J. P. McCormack, and T. F. Hogan (2006), Imaging gravity waves in lower stratospheric AMSU-A radiances, Part 2: Validation case study, Atmos. Chem. Phys., 6, 3343-3362, https://doi.org/10.5194/acp-6-3343-2006.

Eckermann, S. D., L. Hoffmann, M. Höpfner, D. L. Wu, and M. J. Alexander (2009), Antarctic NAT PSC belt of June 2003: Observational validation of the mountain wave seeding hypothesis, Geophys. Res. Lett., 36, L02807, doi: 10.1029/2008GL036629.

Elrod, M. J., R. E. Koch, J. E. Kim, and M. Molina (1995), $\mathrm{HCl}$ vapour pressures and reaction probabilities for $\mathrm{ClONO}_{2}+\mathrm{HCl}$ on liquid $\mathrm{H}_{2} \mathrm{SO}_{4}-\mathrm{HNO}_{3}-\mathrm{HCl}-\mathrm{H}_{2} \mathrm{O}$ solutions, Faraday Discuss., 100, 269-278, doi: 10.1039/FD9950000269.

Engel, I., B. P. Luo, M. C. Pitts, L. R. Poole, C. R. Hoyle, J.-U. Grooß, A. Dörnbrack, and T. Peter (2013), Heterogeneous formation of polar stratospheric clouds - Part 2: Nucleation

This article is protected by copyright. All rights reserved. 
of ice on synoptic scales, Atmos. Chem. Phys., 13 (21), 10,769-10,785, doi:10.5194/acp13-10769-2013.

Engel, I., B. P. Luo, S. M. Khaykin, F. G. Wienhold, H. Vömel, R. Kivi, C. R. Hoyle, J.-U. Grooß, M. C. Pitts, and T. Peter (2014), Arctic stratospheric dehydration - part 2: Microphysical modeling, Atmos. Chem. Phys., 14 (7), 3231-3246, doi:10.5194/acp-143231-2014.

Eyring, V., N. Butchart, D. W. Waugh, H. Akiyoshi, J. Austin, S. Bekki, G. E. Bodeker, B. A. Boville, C. Brühl, M. P. Chipperfield, E. Cordero, M. Dameris, M. Deushi, V. E. Fioletov, S. M. Frith, R. R. Garcia, A. Gettelman, M. A. Giorgetta, V. Grewe, L. Jourdain, D. E. Kinnison, E. Mancini, E. Manzini, M. Marchand, D. R. Marsh, T. Nagashima, E. Nielsen, P. A. Newman, S. Pawson, G. Pitari, D. A. Plummer, E.

Rozanov, M. Schraner, T. G. Shepherd, K. Shibata, R. S. Stolarski, H. Struthers, W. Tian, and M. Yoshiki (2006), Assessment of temperature, trace species and ozone in chemistryclimate simulations of the recent past, J. Geophys. Res., 111 (D22), D22308, doi:10.1029/2006JD007327.

Eyring, V., I. Cionni, G. E. Bodeker, A. J. Charlton-Perez, D. E. Kinnison, J. F. Scinocca, D. W. Waugh, H. Akiyoshi, S. Bekki, M. P. Chipperfield, M. Dameris, S. Dhomse, S. M. Frith, H. Garny, A. Gettelman, A. Kubin, U. Langematz, E. Mancini, M. Marchand, T. Nakamura, L. D. Oman, S. Pawson, G. Pitari, D. A. Plummer, E. Rozanov, T. G. Shepherd, K. Shibata, W. Tian, P. Braesicke, S. C. Hardiman, J. F. Lamarque, O. Morgenstern, J. A. Pyle, D. Smale, and Y. Yamashita (2010), Multi-model assessment of stratospheric ozone return dates and ozone recovery in CCMVal-2 models, Atmos. Chem. Phys., 10 (19), 9451-9472, doi:10.5194/acp-10-9451-2010.

Fahey, D. W., K. K. Kelly, G. V. Ferry, L. R. Poole, J. C. Wilson, D. M. Murphy, M. Loewenstein, and K. R. Chan (1989), In situ measurements of total reactive nitrogen, total water, and aerosol in a polar stratospheric cloud in the Antarctic, J. Geophys. Res., 94 (D9), 11299- 11315, doi:10.1029/JD094iD09p11299.

Fahey, D. W., K. K. Kelly, S. R. Kawa, A. F. Tuck, M. Loewenstein, K. R. Chan, and L. E. Heid (1990a), Observations of denitrification and dehydration in the winter polar stratosphere, Nature, 344, 321-324, doi: 10.1038/344321a0.

Fahey, D. W., S. Solomon, S. R. Kawa, M. Loewenstein, J. R. Podolske, S. E. Strahan, and K. R. Chan (1990b), A diagnostic for denitrification in the winter polar stratospheres, Nature, 345, 698-702, doi: 10.1038/345698a0.

Fahey, D. W., R. S. Gao, K. S. Carslaw, J. Kettleborough, P. J. Popp, M. J. Northway, J. C. Holecek, S. C. Ciciora, R. J. McLaughlin, T. L. Thompson, R. H. Winkler, D. G. Baumgardner, B. Gandrud, P. O. Wennberg, S. Dhaniyala, K. McKinney, Th. Peter, R. J. Salawitch, T. P. Bui, J. W. Elkins, C. R. Webster, E. L. Atlas, H. Jost, J. C. Wilson, R. L. Herman, A. Kleinböhl, and M. von König, (2001), The Detection of Large $\mathrm{HNO}_{3}$ -

This article is protected by copyright. All rights reserved. 
Containing Particles in the Winter Arctic Stratosphere, Science, 291, 1026-1031, doi: $10.1126 /$ science. 1057265 .

Farman, J. C., B. G. Gardiner, and J. D. Shanklin (1985), Large losses of total ozone in Antarctica reveal seasonal $\mathrm{ClO}_{\mathrm{x}} / \mathrm{NO}_{\mathrm{x}}$ interaction, Nature, 315, 207-210, doi: $10.1038 / 315207 \mathrm{a} 0$.

Farmer, C., Toon, G., Schaper, P. et al. (1987), Stratospheric trace gases in the spring 1986 Antarctic atmosphere, Nature, 329, 126-130, doi:10.1038/329126a0.

Feng, W., M. P. Chipperfield, S. Davies, G. W. Mann, K. S. Carslaw, S. Dhomse, L. Harvey, C. Randall, and M. L. Santee (2011), Modelling the effect of denitrification on polar ozone depletion for Arctic winter 2004/2005, Atmos. Chem. Phys., 11 (13), 6559-6573, doi:10.5194/acp-11-6559-2011.

Fikke, S. M., J. E. Kristjánsson, and Ø. Nordli (2017), Screaming clouds, Weather, 72 (5), 115121, doi:10.1002/wea.2786.

Fischer, H., M. Birk, C. Blom, B. Carli, M. Carlotti, T. von Clarmann, L. Delbouille, A. Dudhia, D. Ehhalt, M. Endemann, J. M. Flaud, R. Gessner, A. Kleinert, R. Koopmann, J. Langen, M. López-Puertas, P. Mosner, H. Nett, H. Oelhaf, G. Perron, J. Remedios, M. Ridolfi, G. Stiller, , and R. Zander (2008), MIPAS: An instrument for atmospheric and climate research, Atmos. Chem. Phys., 8, doi:10.5194/acp-8-2151-2008.

Fortin, T. J., K. Drdla, L. T. Iraci, and M. A. Tolbert (2003), Ice condensation on sulfuric acid tetrahydrate: Implications for polar stratospheric ice clouds, Atmos. Chem. Phys., 3 (4), 987-997, doi:10.5194/acp-3-987-2003.

Fromm, M. D., J. D. Lumpe, R. M. Bevilacqua, E. P. Shettle, J. Hornstein, S. T. Massie, and K. H. Fricke (1997), Observations of Antarctic polar stratospheric clouds by POAM II: 1994-1996, J. Geophys. Res., 102, 23,659-23,672.

Fromm, M. D., R. M. Bevilacqua, J. Hornstein, E. Shettle, K. Hoppel, and J. D. Lumpe (1999), An analysis of Polar Ozone and Aerosol Measurement (POAM) II Arctic polar stratospheric cloud observations, 1993- 1996, J. Geophys. Res., 104, 24,341-24,357.

Fromm, M., J. Alfred, and M. Pitts (2003), A unified, long-term, high-latitude stratospheric aerosol and cloud database using SAM II, SAGE II, and POAM II/III data: Algorithm description, database definition, and climatology, J. Geophys. Res., 108, 4366, doi:10.1029/2002JD002772.

Fueglistaler, S., B. P. Luo, C. Voigt, K. S. Carslaw, and T. Peter (2002a), NAT-rock formation by mother clouds: A microphysical model study, Atmos. Chem. Phys., 2, 93-98, doi: 10.5194/acp-2-93-2002.

Fueglistaler, S., B. P. Luo, S. Buss, H. Wernli, C. Voigt, M. Müller, R. Neuber, C. A. Hostetler, L. Poole, H. Flentje, D. W. Fahey, M. J. Northway, and T. Peter (2002b), Large NAT

This article is protected by copyright. All rights reserved. 
particle formation by mother clouds: Analysis of SOLVE/THESEO-2000 observations, Geophys. Res. Lett., 29 (12), 1610, doi: 10.1029/2001GL014548.

Fueglistaler, S., S. Buss, B. P. Luo, H. Wernli, H. Flentje, C. A. Hostetler, L. R. Poole, K. S. Carslaw, and T. Peter (2003), Detailed modeling of mountain wave PSCs, Atmos. Chem. Phys., 3, 697-712, https://doi.org/10.5194/acp-3-697-2003.

Gary, B. L. (2006), Mesoscale temperature fluctuations in the stratosphere, Atmos. Chem. Phys., 6, 4577-4589, doi:10.5194/acp-6-4577-2006.

Gary, B. L. (2008), Mesoscale temperature fluctuations in the Southern Hemisphere stratosphere, Atmos. Chem. Phys., 8, 4677-4681, doi:10.5194/acp-8-4677-2008.

Gobbi, G. P. (1995), Lidar estimation of stratospheric aerosol properties: Surface, volume, and extinction to backscatter ratio, J. Geophys. Res. A, 100 (D6), 11,219-11,235, doi:10.1029/94JD03106.

Goodman, J., S. Verma, R. F. Pueschel, P. Hamill, G. V. Ferry, and D. Webster (1997), New evidence of size and composition of polar stratospheric cloud particles, Geophys. Res. Lett., 24 (5), 615-618, doi:10.1029/97GL00256.

Grooß, J.-U., G. Günther, R. Müller, P. Konopka, S. Bausch, H. Schlager, C. Voigt, C. M. Volk, and G. C. Toon (2005), Simulation of denitrification and ozone loss for the Arctic winter 2002/2003, Atmos. Chem. Phys., 5, 1437-1448, doi: 10.5194/acp-5-1437-2005.

Grooß, J.-U., K. Brautzsch, R. Pommrich, S. Solomon, and R. Müller (2011), Stratospheric ozone chemistry in the Antarctic: What determines the lowest values that can be reached and their recovery?, Atmos. Chem. Phys., 11, 12,217-12,226, doi: 10.5194/acp-11-122172011.

Grooß, J.-U., I. Engel, S. Borrmann, W. Frey, G. Günther, C. R. Hoyle, R. Kivi, B. P. Luo, S. Molleker, T. Peter, M. C. Pitts, H. Schlager, G. Stiller, H. Vömel, K. A. Walker, and R. Müller (2014), Nitric acid trihydrate nucleation and denitrification in the Arctic stratosphere, Atmos. Chem. Phys., 14 (2), 1055-1073, doi:10.5194/acp-14-1055-2014.

Grooß, J.-U., R. Müller, R. Spang, I. Tritscher, T. Wegner, M. P. Chipperfield, W. Feng, D. E. Kinnison, and S. Madronich (2018), On the discrepancy of $\mathrm{HCl}$ processing in the core of

This article is protected by copyright. All rights reserved. 
the wintertime polar vortices, Atmos. Chem. Phys., pp. 8647-8666, doi:10.5194/acp-188647-2018.

Grothe, H., H. Tizek, D. Waller, and D. J. Stokes (2006), The crystallization kinetics and morphology of nitric acid trihydrate, Phys. Chem. Chem. Phys., 8, 2232-2239, doi:10.1039/B601514J.

Grothe, H., H. Tizek, and I. K. Ortega (2008), Metastable nitric acid hydrates- possible constituents of polar stratospheric clouds?, Faraday Discuss., 137, 223-234, doi:10.1039/B702343J.

Hanson, D. (2003), Reactivity of $\mathrm{BrONO}_{2}$ and $\mathrm{HOBr}$ on sulfuric acid solutions at low temperatures, J. Geophys. Res., 108 (D8), doi:10.1029/2002JD002519.

Hanson, D. R., and K. Mauersberger (1988), Laboratory studies of the nitric acid trihydrate: Implications for the south polar stratosphere, Geophys. Res. Lett., 15 (8), 855-858, doi:10.1029/GL015i008p00855.

Hanson, D. R., and A. R. Ravishankara (1991), The reaction probabilities of $\mathrm{ClONO}_{2}$ and $\mathrm{N}_{2} \mathrm{O}_{5}$ on 40 to $75 \%$ sulfuric acid solutions, J. Geophys. Res., 96, 17,307-17,314, doi: 10.1029/91JD01750.

Hanson, D. R., and A. R. Ravishankara (1993), Reaction of $\mathrm{ClONO}_{2}$ with $\mathrm{HCl}$ on NAT, NAD, and frozen sulfuric acid and hydrolysis of $\mathrm{N}_{2} \mathrm{O}_{5}$ and $\mathrm{ClONO}_{2}$ on frozen sulfuric acid, $J$. Geophys. Res., 98, 22,931-22,936, doi: 10.1029/93JD01929.

Hanson, D. R., and A. R. Ravishankara (1994), Reactive uptake of $\mathrm{ClONO}_{2}$ onto sulfuric acid due to reaction with $\mathrm{HCl}$ and $\mathrm{H}_{2} \mathrm{O}, J$. Phys. Chem., 98, 5728-5735, doi: $10.1021 / \mathrm{j} 100073 \mathrm{a} 026$.

Hanson, D. R., A. R. Ravishankara, and E. R. Lovejoy (1996), Reaction of $\mathrm{BrONO}_{2}$ with $\mathrm{H}_{2} \mathrm{O}$ on submicron sulfuric acid aerosol and the implications for the lower stratosphere, $J$. Geophys. Res., 101, 9063-9069, doi:10.1029/96JD00347.

Hildebrandson, J. V. H. (1895), Ausserordentliche Wolkenhöhe und irisirende Wolken, Meteorologische Zeitschrift, 12, 71-72.

Hitchman, M. H., M. L. Buker, G. J. Tripoli, E. V. Browell, W. B. Grant, T. J. McGee, and J. F. Burris (2003), Nonorographic generation of Arctic polar stratospheric clouds during December 1999, J. Geophys. Res., 108 (D5), 8325, doi: 10.1029/2001JD001034.

Hoffmann, L., R. Spang, A. Orr, M. J. Alexander, L. A. Holt, and O. Stein (2017), A decadal satellite record of gravity wave activity in the lower stratosphere to study polar

This article is protected by copyright. All rights reserved. 
stratospheric cloud formation, Atmos. Chem. Phys., 17 (4), 2901-2920, doi:10.5194/acp17-2901-2017.

Holton, J.R., and G.J. Hakim, An Introduction to Dynamic Meteorology, Fifth Edition, Academic Press, Elsevier, Amsterdam, ISBN 978-0-12-384866-6.

Höpfner, M. (2004), Study on the impact of polar stratospheric clouds on high resolution mid-IR limb emission spectra, J. Quant. Spectr. Radiat. Transfer, 83 (1), 93-107, doi:10.1016/S0022-4073(02)00299-6.

Höpfner, M., B. P. Luo, P. Massoli, F. Cairo, R. Spang, M. Snels, G. Di Donfrancesco, G. Stiller, T. von Clarmann, H. Fischer, and U. Biermann (2006a), Spectroscopic evidence for NAT, STS, and ice in MIPAS infrared limb emission measurements of polar stratospheric clouds, Atmos. Chem. Phys., 6, 1201-1219, doi: 10.5194/acp-6-1201-2006.

Höpfner, M., N. Larsen, R. Spang, B. P. Luo, J. Ma, S. H. Svendsen, S. D. Eckermann, B. Knudsen, P. Massoli, F. Cairo, G. Stiller, T. Von Clarmann, and H. Fischer (2006b), MIPAS detects Antarctic stratospheric belt of NAT PSCs caused by mountain waves, Atmos. Chem. Phys., 6, 1221-1230, doi: 10.5194/acp-6-1221-2006.

Höpfner, M., M. C. Pitts, and L. R. Poole (2009), Comparison between CALIPSO and MIPAS observations of polar stratospheric clouds, J. Geophys. Res., 114, D00H05, doi:10.1029/2009JD012114.

Höpfner, M., T. Deshler, M. Pitts, L. Poole, R. Spang, G. Stiller, and T. von Clarmann (2018), The MIPAS/Envisat climatology (2002-2012) of polar stratospheric cloud volume density profiles, Atmos. Meas. Tech., 11 (10), 5901-5923, doi:10.5194/amt-11-59012018.

Hoyle, C. R., B. P. Luo, and T. Peter (2005), The origin of high ice crystal number densities in cirrus clouds, J. Atmos. Sci., 62 (7, Part 2), 2568-2579, doi: 10.1175/JAS3487.1.

Hoyle, C. R., I. Engel, B. P. Luo, M. C. Pitts, L. R. Poole, J.-U. Grooß, and T. Peter (2013), Heterogeneous formation of polar stratospheric clouds - Part 1: Nucleation of nitric acid

This article is protected by copyright. All rights reserved. 
trihydrate (NAT), Atmos. Chem. Phys., 13 (18), 9577-9595, doi:10.5194/acp-13-95772013.

Hülsmann O. and W. Biltz, Über die thermische Analyse des Systems Schwefelsäure/Wasser, Z. anorg. Chem., 218, 369-378, 1934.

Iraci, L. T., A. M. Middlebrook, and M. A. Tolbert (1995), Laboratory studies of the formation of polar stratospheric clouds: Nitric acid condensation on thin sulfuric acid films, $J$. Geophys. Res., 100, 20,969-20,977, doi: 10.1029/95JD02267.

Iwasaka, Y. (1985), Lidar measurement of the stratospheric aerosol layer at Syowa Station $\left(69^{\circ} 00^{\prime} \mathrm{S}, 39^{\circ} 35^{\prime} \mathrm{E}\right)$, Antarctica, J. Meteorol. Soc. Japan 63, 283-287, doi: 10.2151/jmsj1965.63.2_283.

Iwasaka, Y. (1986), Non-spherical particles in the Antarctic polar stratosphere- increase in particulate content and stratospheric water vapor budget, Tellus B, 38B (5), 364-374, doi:10.1111/j.1600-0889.1986.tb00261.x.

James, A. D., J. S. A. Brooke, T. P. Mangan, T. F. Whale, J. M. C. Plane, and B. J. Murray (2018), Nucleation of nitric acid hydrates in polar stratospheric clouds by meteoric material, Atmos. Chem. Phys., 18 (7), 4519-4531, doi: 10.5194/acp-18-4519-2018.

Jourdain, L., S. Bekki, F. Lott, and F. Lefevre (2008), The coupled chemistry-climate model LMDz-REPROBUS: Description and evaluation of a transient simulation of the period 1980-1999, Ann. Geophys., 26 (6), 1391-1413, doi: 10.5194/angeo-26-1391-2008.

Junge, C. E., C. W. Changnon, and J. E. Manson (1961a), Stratospheric aerosols, J Meteorol, 18, 81-108, doi: 10.1175/1520-0469(1961)018\%3C0081:SA\%3E2.0.CO;2.

Junge, C. E., C. W. Changnon, and J. E. Manson (1961b), A world-wide stratospheric aerosol layer, Science, 133, 1478-1479, doi:10.1126/science.133.3463.1478-a.

Kaufmann, M., J. Blank, T. Guggenmoser, J. Ungermann, A. Engel, M. Ern, F. Friedl-Vallon, D. Gerber, J. U. Grooß, G. Guenther, M. Höpfner, A. Kleinert, E. Kretschmer, T. Latzko, G. Maucher, T. Neubert, H. Nordmeyer, H. Oelhaf, F. Olschewski, J. Orphal, P. Preusse, H. Schlager, H. Schneider, D. Schuettemeyer, F. Stroh, O. Suminska-Ebersoldt, B. Vogel, C. M. Volk, W. Woiwode, and M. Riese (2015), Retrieval of three-dimensional small-scale structures in upper- tropospheric/lower-stratospheric composition as measured by GLORIA, Atmos. Meas. Tech., 8 (1), 81-95, doi:10.5194/amt-8-81-2015.

Khosrawi, F., J. Urban, M. C. Pitts, P. Voelger, P. Achtert, M. Kaphlanov, M. L. Santee, G. L. Manney, D. Murtagh, and K.-H. Fricke (2011), Denitrification and polar stratospheric cloud formation during the Arctic winter 2009/2010, Atmos. Chem. Phys., 11 (16), 84718487, doi:10.5194/acp-11-8471-2011.

Khosrawi, F., O. Kirner, G. Stiller, M. Höpfner, Santee, M. L., S. Kellmann, and P. Braesicke (2018), Comparison of ECHAM5/MESSy Atmospheric Chemistry (EMAC) simulations of the Arctic winter 2009/2010 and 2010/2011 with Envisat/MIPAS and Aura/MLS

This article is protected by copyright. All rights reserved. 
observations, Atmos. Chem. Phys., 18, 8873-8892, https://doi.org/10.5194/acp-18-88732018.

Kirner, O., R. Ruhnke, J. Buchholz-Dietsch, P. Jöckel, C. Brühl, and B. Steil (2011), Simulation of polar stratospheric clouds in the chemistry-climate-model EMAC via the submodel PSC, Geosci. Model Dev., 4 (1), 169-182, doi:10.5194/gmd-4- 169-2011.

Kirner, O., R. Müller, R. Ruhnke, and H. Fischer (2015), Contribution of liquid, NAT and ice particles to chlorine activation and ozone depletion in Antarctic winter and spring, Atmos. Chem. Phys., 15 (4), 2019-2030, doi:10.5194/acp-15-2019-2015.

Knopf, D. A. (2006), Do NAD and NAT form in liquid stratospheric aerosols by pseudoheterogeneous nucleation?, J. Phys. Chem., 110 (17), 5745-5750, doi: 10.1021/jp055376j, pMID: 16640368.

Koehler, B. G., A. M. Middlebrook, and M. A. Tolbert (1992), Characterization of model polar stratospheric cloud films using Fourier transform infrared spectroscopy and temperature programmed desorption, J. Geophys. Res., 97 (D8), 8065-8074, doi:10.1029/91JD01844.

Kohma, M., and K. Sato (2013), Simultaneous occurrence of polar stratospheric clouds and upper-tropospheric clouds caused by blocking anticyclones in the Southern Hemisphere, Atmos. Chem. Phys., 13, 3849-3864, doi: 10.5194/acp-13-3849-2013.

Konopka, P., Steinhorst, H.-M., Grooß, J.-U., Günther, G., R., M., Elkins, J. W., Jost, H.-J., Richard, E., Schmidt, U., Toon, G., and McKenna, D. S. (2004), Mixing and ozone loss in the 1999-2000 Arctic vortex: Simulations with the three-dimensional Chemical Lagrangian Model of the Stratosphere CLaMS, J. Geophys. Res., 109, D02315, doi:10.1029/2003JD003792.

Koop, T., and K. S. Carslaw (1996), Melting of $\mathrm{H}_{2} \mathrm{SO}_{4} \cdot 4 \mathrm{H}_{2} \mathrm{O}$ particles upon cooling: Implications for polar stratospheric clouds, Science, 272 (5268), 1638-1641, doi:10.1126/science.272.5268.1638.

Koop, T., U. M. Biermann, W. Raber, B. P. Luo, P. J. Crutzen, and T. Peter (1995), Do stratospheric aerosol droplets freeze above the ice frost point?, Geophys. Res. Lett., 22 (8), 917-920, doi:10.1029/95GL00814.

Koop, T., K. S. Carslaw, and T. Peter (1997a), Thermodynamic stability and phase transitions of PSC particles, Geophys. Res. Lett., 24 (17), 2199-2202, doi: 10.1029797GL02148.

Koop, T., B. P. Luo, U. M. Biermann, P. J. Crutzen, and T. Peter (1997b), Freezing of $\mathrm{HNO}_{3} / \mathrm{H}_{2} \mathrm{SO}_{4} / \mathrm{H}_{2} \mathrm{O}$ solutions at stratospheric temperatures: Nucleation statistics and experiments, J. Phys. Chem. A, 101, 1117-1133, doi: 10.1021/jp9626531.

Koop, T., H. P. Ng, L. T. Molina, and M. J. Molina (1998), A new optical technique to study aerosol phase transitions: The nucleation of ice from $\mathrm{H}_{2} \mathrm{SO}_{4}$ aerosols, J. Phys. Chem. A, 102 (45), 8924-8931, doi:10.1021/jp9828078.

Koop, T., B. P. Luo, A. Tsias, and T. Peter (2000), Water activity as the determinant for homogeneous ice nucleation in aqueous solutions, Nature, 406, 611-614, doi: $10.1038 / 35020537$.

Lambert, A., and M. L. Santee (2018), Accuracy and precision of polar lower stratospheric temperatures from reanalyses evaluated from A-Train CALIOP and MLS, COSMIC GPS

This article is protected by copyright. All rights reserved. 
$\mathrm{RO}$, and the equilibrium thermodynamics of supercooled ternary solutions and ice clouds, Atmos. Chem. Phys., 18 (3), 1945-1975, doi:10.5194/acp- 18-1945-2018.

Lambert, A., W. G. Read, N. J. Livesey, M. L. Santee, G. L. Manney, L. Froideveaux, D. L. Wu, M. J. Schwartz, H. C. Pumphrey, C. Jimenez, G. E. Nedoluha, R. E. Cofield, D. T. Cuddy, W. H. Daffer, B. J. Drouin, R. Fuller, R. F. Jarnot, B. W. Knosp, H. M. Pickett, V. S. Perun, W. V. Snyder, P. C. Stek, R. P. Thurstans, P. A. Wagner, J. W. Waters, K. W. Jucks, G. C. Toon, R. A. Stachnik, P. F. Bernath, C. D. Boone, K. A. Walker, J. Urban, D. Murtagh, J. W. Elkins, and E. Atlas (2007), Validation of the Aura Microwave Limb Sounder middle atmosphere water vapor and nitrous oxide measurements, $J$. Geophys. Res., 112 (D24), 24S36, doi:10.1029/2007JD008724.

Lambert, A., M. L. Santee, D. L. Wu, and J. H. Chae (2012), A-train CALIOP and MLS observations of early winter Antarctic polar stratospheric clouds and nitric acid in 2008, Atmos. Chem. Phys., 12 (6), 2899-2931, doi:10.5194/acp-12-2899-2012.

Lambert, A., M. L. Santee, and N. J. Livesey (2016), Interannual variations of early winter Antarctic polar stratospheric cloud formation and nitric acid observed by CALIOP and MLS, Atmos. Chem. Phys., 16 (23), 15,219-15,246, doi:10.5194/acp-16- 15219-2016.

Langematz, U., S. Meul, K. Grunow, E. Romanowsky, S. Oberländer, J. Abalichin, and A. Kubin (2014), Future Arctic temperature and ozone: The role of stratospheric composition changes, J. Geophys. Res. Atmos., 119, 2092-2112, doi:10.1002/2013JD021100.

Larsen, N., S. Hoyer, Svendsen, B. M. Knudsen, I. S. Mikkelsen, C. Voigt, A. Kohlmann, J. Schreiner, K. Mauersberger, T. Deshler, C. Kröger, J. M. Rosen, N. T. Kjome, A. Adriani, F.Cairo, G. Di Donfrancesco, J. Ovarlez, H. Ovarlez, A. Dörnbrack, and T. Birner (2002), Microphysical mesoscale simulations of polar stratospheric cloud formation constrained by in situ measurements of chemical and optical cloud properties, J. Geophys. Res., 107, 8301, doi:10.1029/2001JD000999.

Larsen, N., B. M. Knudsen, S. H. Svendsen, T. Deshler, J. M. Rosen, R. Kivi, C. Weisser, J. Schreiner, K. Mauersberger, F. Cairo, J. Ovarlez, H. Oelhaf, and R. Spang (2004), Formation of solid particles in synoptic-scale Arctic PSCs in early winter 2002/2003, Atmos. Chem. Phys., 4, 2001-2013, doi: 10.5194/acp-4-2001-2004.

Lary, D. J., M. P. Chipperfield, R. Toumi, and T. Lenton (1996), Heterogeneous atmospheric bromine chemistry, J. Geophys. Res., 101, 1489-1504, doi: 10.1029/95JD02839.

Lawrence, Z. D., G. L. Manney, K. Minschwaner, M. L. Santee, and A. Lambert (2015), Comparisons of polar processing diagnostics from 34 years of the ERA-Interim and

This article is protected by copyright. All rights reserved. 
MERRA reanalyses, Atmos. Chem. Phys., 15, 3873-3892, doi:10.5194/acp-15-38732015.

Lefèvre, F., F. Figarol, K. S. Carslaw, and T. Peter (1998), The 1997 Arctic ozone depletion quantified from three-dimensional model simulations, Geophys. Res. Lett., 25(13), 24252428, doi:10.1029/98GL51812.

Leu, M. T. (1988), Laboratory studies of sticking coefficients and heterogeneous reactions important in the Antarctic stratosphere, Geophys. Res. Lett., 15 (1), 17-20, doi:10.1029/GL015i001p00017.

Livesey, N. J., W. V. Snyder, W. G. Read, and P. Wagner (2006), Retrieval algorithms for the EOS Microwave Limb Sounder (MLS) instrument, IEEE Trans. Geosci. Remote Sens., 44, 1144-1155, doi: 10.1109/TGRS.2006.872327.

Livesey, N. J., W. G. Read, P. A. Wagner, L. Froidevaux, A. Lambert, G. L. Manney, L. F. M. Valle, H. C. Pumphrey, M. L. Santee, M. J. Schwartz, S. Wang, R. A. Fuller, R. F. Jarnot, B. W. Knosp, and E. Martinez (2017), Version 4.2x level 2 data quality and description document, JPL D-33509 Rev. C.

Lowe, D., and A. R. MacKenzie (2008), Polar stratospheric cloud microphysics and chemistry, $J$. Atmos. Solar Terr. Phys., 70 (1), 13-40, doi: 10.1016/j.jastp.2007.09.011.

Luo, B. P., T. Peter, and P. Crutzen (1994), Freezing of stratospheric aerosol droplets, Geophys. Res. Lett., 21, (13), 1447-1450, doi: 10.1029/93GL03076.

Luo, B. P., C. Voigt, S. Fueglistaler, and T. Peter (2003), Extreme NAT supersaturations in mountain wave ice PSCs: A clue to NAT formation, J. Geophys. Res., 108, 4443, doi:10.1029/2002JD003104.

MacKenzie, A. R., M. Kulmala, A. Laaksonen, and T. Vesala (1995), On the theories of type 1 polar stratospheric cloud formation, J. Geophys. Res., 100, 11,275- 11,288, doi: https://doi.org/10.1029/95JD00699.

Mann, G. W., S. Davies, K. S. Carslaw, M. P. Chipperfield, and J. Kettleborough (2002), Polar vortex concentricity as a controlling factor in Arctic denitrification, J. Geophys. Res., 107, 4663, doi:10.1029/2002JD002102.

Mann, G. W., K. S. Carslaw, M. P. Chipperfield, S. Davies, and S. D. Eckermann (2005), Large nitric acid trihydrate particles and denitrification caused by mountain waves in the Arctic stratosphere, J. Geophys. Res., 110 (D8), D08202, doi: 10.1029/2004JD005271.

Manney, G. L., M. L. Santee, M. Rex, N. J. Livesey, M. C. Pitts, P. Veefkind, E. R. Nash, I. Wohltmann, R. Lehmann, L. Froidevaux, L. R. Poole, M. R. Schoeberl, D. P. Haffner, J. Davies, V. Dorokhov, H. Gernandt, B. Johnson, R. Kivi, E. Kyrö, N. Larsen, P. F. Levelt, A. Makshtas, C. T. McElroy, H. Nakajima, M. C. Parrondo, D. W. Tarasick, P. von der Gathen, K. A. Walker, and N. S. Zinoviev (2011), Unprecedented Arctic ozone loss in 2011, Nature, 478, 469-475, doi:10.1038/nature10556.

Marchand, M., P. Keckhut, S. Lefebvre, C. Claud, D. Cugnet, A. Hauchecorne, F. Lefevre, M. P. Lefebvre, J. Jumelet, F. Lott, F. Hourdin, G. Thuillier, V. Poulain, S. Bossay, P. Lemennais, C. David, and S. Bekki (2012), Dynamical amplification of the stratospheric

This article is protected by copyright. All rights reserved. 
solar response simulated with the Chemistry- Climate Model LMDz-Reprobus, J. Atmos. Solar Terr. Phys., 75, 147-160, doi: 10.1016/j.jastp.2011.11.008.

Marcolli, C., S. Gedamke, T. Peter, and B. Zobrist (2007), Efficiency of immersion mode ice nucleation on surrogates of mineral dust, Atmos. Chem. Phys., 7 (19), 5081-5091, doi:10.5194/acp-7-5081-2007.

Massie, S. T., J. E. Dye, D. Baumgardner, W. J. Randel, F. Wu, X. Tie, L. Pan, F. Figarol, G. P. Brasseur, M. L. Santee, W. G. Read, R. G. Grainger, A. Lambert, J. L. Mergenthaler, and A. Tabazadeh (1997), Simultaneous observations of polar stratospheric clouds and HNO3 over Scandinavia in January,1992, Geophys. Res. Lett., 24(5), 598, doi: 10.1029/97GL00315.

Massoli, P., M. Maturilli, and R. Neuber (2006), Climatology of Arctic polar stratospheric clouds as measured by lidar in Ny-Ålesund, Spitsbergen $\left(79 \circ \mathrm{N}, 12^{\circ} \mathrm{E}\right)$, J. Geophys. Res. A, 111 (D9), doi:10.1029/2005JD005840.

Maturilli, M., R. Neuber, P. Massoli, F. Cairo, A. Adriani, M. L. Moriconi, and G. D. Donfrancesco (2005), Differences in Arctic and Antarctic PSC occurrence as observed by lidar in Ny-Ålesund (79॰ N, 12॰ E) and McMurdo (78॰ S, 167॰ E), Atmos. Chem. Phys., 5, 2081-2090, doi: 10.5194/acp-5-2081-2005.

McCormick, M. P., and C. R. Trepte (1987), Polar stratospheric optical depth observed between 1978 and 1985, J. Geophys. Res. A, 92 (D4), 4297-4306, doi:10.1029/JD092iD04p04297.

McCormick, M. P., H. M. Steele, P. Hamill, W. P. Chu, and T. J. Swissler (1982), Polar stratospheric cloud sightings by SAM II, J. Atmos. Sci., 39, 1387-1397, doi: 10.1175/1520-0469(1982)039\%3C1387:PSCSBS\%3E2.0.CO;2.

McCormick, M. P., W. P. Chu, G. W. Grams, P. Hamill, B. M. Herman, L. R. McMaster, T. J. Pepin, P. B. Russell, H. M. Steele, and T. Swissler (1981), High- latitude stratospheric aerosols measured by the SAM-II satellite system in 1978 and 1979, Science, 214 (4518), 328-331, doi:10.1126/science.214.4518.328.

McCormick, M. P., P. H. Wang, and M. C. Pitts (1993), Background stratospheric aerosol and polar stratospheric cloud reference models, Adv. Space Res., 13, 7-29, doi: https://doi.org/10.1016/0273-1177(93)90003-T.

McDonald, A. J., S. E. George, and R. M. Woollands (2009), Can gravity waves significantly impact PSC occurrence in the Antarctic?, Atmos. Chem. Phys., 9, 8825-8840, doi: 10.5194/acp-9-8825-2009.

McElroy, M. B., R. J. Salawitch, S. C. Wofsy, and J. A. Logan (1986a), Antarctic ozone: Reductions of Antarctic ozone due to synergistic interactions of chlorine and bromine, Nature, 321, 759-762, doi: 10.1038/321759a0.

McElroy, M. B., R. J. Salawitch, and S. C. Wofsy (1986b), Antarctic O 3 - chemical mechanisms for the spring decrease, Geophys. Res. Lett., 13, 1296-1299, doi: 10.1029/GL013i012p01296.

McKenna, D. S., Konopka, P., Grooß, J.-U., Gunther, G., Müller, R., Spang, R., Offermann, D., and Orsolini, Y. (2002a), A new Chemical Lagrangian Model of the Stratosphere

This article is protected by copyright. All rights reserved. 
(CLaMS): 1. Formulation of advection and mixing, J. Geophys. Res., 107, 4309, doi: 10.1029/2000JD000114.

McKenna, D. S., Grooß, J.-U., Günther, G., Konopka, P., Müller, R., Carver, G., and Sasano, Y. (2002b), A new Chemical Lagrangian Model of the Stratosphere (CLaMS): Part II Formulation of chemistry-scheme and initialisation, J. Geophys. Res., 107, 10.1029/2000JD000 113.

Meilinger, S., K. Koop, B. P. Luo, T. Huthwelker, K. S. Carslaw, U. Krieger, P. J. Crutzen, and T. Peter (1995), Size-dependent stratospheric droplet composition in lee wave temperature fluctuations and their potential role in PSC freezing, Geophys. Res. Lett., 22 (22), 3031-3034, doi:10.1029/95GL03056.

Middlebrook, A. M., L. T. Iraci, L. S. McNeill, B. G. Koehler, M. A. Wilson, O. W. Saastad, M. A. Tolbert, and D. R. Hanson (1993), Fourier transform-infrared studies of thin $\mathrm{H}_{2} \mathrm{SO}_{4} / \mathrm{H}_{2} \mathrm{O}$ films: Formation, water uptake, and solid-liquid phase changes, J. Geophys. Res., 98 (D11), 20,473-20,481, doi:10.1029/93JD02454.

Möhler, O., H. Bunz, and O. Stetzer (2006), Homogeneous nucleation rates of nitric acid dihydrate (NAD) at simulated stratospheric conditions - Part II: Modelling, Atmos. Chem. Phys., 6 (10), 3035-3047, doi:10.5194/acp-6-3035-2006.

Mohn, H. (1895), Perlemorskyer, Naturen, 19, 257-267.

Molina, M. J., and F. S. Rowland (1974), Stratospheric sink for chlorofluoromethanes - chlorine atom-catalyzed destruction of ozone, Nature, 249 (5460), 810-812, doi:10.1038/249810a0.

Molina, L. T., and M. J. Molina (1987), Production of $\mathrm{Cl}_{2} \mathrm{O}_{2}$ from the self-reaction of the $\mathrm{ClO}$ radical, J. Phys. Chem., 91, 433-436, doi: 10.1021/j100286a035.

Molina, M. J., R. Zhang, P. J. Wooldridge, J. R. McMahon, J. E. Kim, H. Y. Chang, and K. D. Beyer (1993), Physical chemistry of the $\mathrm{H}_{2} \mathrm{SO}_{4} / \mathrm{HNO}_{3} / \mathrm{H}_{2} \mathrm{O}$ system: Implications for polar stratospheric clouds, Science, 261, 1418-1423, doi: 10.1126/science.261.5127.1418.

Molleker, S., S. Borrmann, H. Schlager, B. Luo, W. Frey, M. Klingebiel, R. Weigel, M. Ebert, V. Mitev, R. Matthey, W. Woiwode, H. Oelhaf, A. Dörnbrack, G. Stratmann, J.-U. Grooß, G. Günther, B. Vogel, R. Müller, M. Krämer, J. Meyer, and F. Cairo (2014), Microphysical properties of synoptic-scale polar stratospheric clouds: In situ measurements of unexpectedly large $\mathrm{HNO}_{3}$-containing particles in the Arctic vortex, Atmos. Chem. Phys., 14 (19), 10,785-10,801, doi:10.5194/acp-14-10785-2014.

Morgenstern, O., M. I. Hegglin, E. Rozanov, F. M. O’Connor, N. L. Abraham, H. Akiyoshi, A. T. Archibald, S. Bekki, N. Butchart, M. P. Chipperfield, M. Deushi, S. S. Dhomse, R. R. Garcia, S. C. Hardiman, L. W. Horowitz, P. Joeckel, B. Josse, D. Kinnison, M. Lin, E. Mancini, M. E. Manyin, M. Marchand, V. Marecal, M. Michou, L. D. Oman, G. Pitari, D. A. Plummer, L. E. Revell, D. Saint-Martin, R. Schofield, A. Stenke, K. Stone, K. Sudo, T. Y. Tanaka, S. Tilmes, Y. Yamashita, K. Yoshida, and G. Zeng (2017), Review

This article is protected by copyright. All rights reserved. 
of the global models used within phase 1 of the Chemistry-Climate Model Initiative (CCMI), Geosci. Model Dev., 10 (2), 639-671, doi:10.5194/gmd-10-639-2017.

Müller, R., and T. Peter (1992), The Numerical Modelling of the Sedimentation of Polar Stratospheric Cloud Particles, Ber. Bunsenges. Chem., 96 (3), 353-361.

Müller, R., J.-U. Grooß, A. M. Zafar, S. Robrecht, and R. Lehmann (2018), The maintenance of elevated active chlorine levels in the Antarctic lower stratosphere through $\mathrm{HCl}$ null cycles, Atmos. Chem. Phys., 18 (4), 2985-2997, doi:10.5194/acp- 18-2985-2018.

Murphy, D. M., and B. I. Gary (1995), Mesoscale temperature fluctuation and polar stratospheric clouds, J. Atmos. Sci., 52, 1753-1760, doi: 10.1175/15200469(1995)052\%3C1753:MTFAPS\%3E2.0.CO;2.

Murphy, D. M., K. D. Froyd, J. P. Schwarz, and J. C. Wilson (2014), Observations of the chemical composition of stratospheric aerosol particles, Q. J. R. Meteorol. Soc., 140 (681), 1269-1278, doi:10.1002/qj.2213.

Nakajima, H., I. Wohltmann, T. Wegner, M. Takeda, M. C. Pitts, L. R. Poole, R. Lehmann, M. L. Santee, and M. Rex, Polar stratospheric cloud evolution and chlorine activation measured by CALIPSO and MLS, and modeled by ATLAS, Atmos. Chem. Phys., 16, 3311-3325, doi:10.5194/acp-16-3311-2016

Noel, V., and M. Pitts (2012), Gravity wave events from mesoscale simulations, compared to polar stratospheric clouds observed from spaceborne lidar over the Antarctic Peninsula, $J$. Geophys. Res., 117, D11207, doi:10.1029/2011JD017318.

Noel, V., A. Hertzog, and H. Chepfer (2009), CALIPSO observations of wave-induced PSCs with near-unity optical depth over Antarctica in 2006-2007, J. Geophys. Res., 114, D05202, doi:10.1029/2008JD010604.

Orr, A., J. S. Hosking, L. Hoffmann, J. Keeble, S. M. Dean, H. K. Roscoe, N. L. Abraham, S. Vosper, and P. Braesicke (2015), Inclusion of mountain-wave-induced cooling for the formation of PSCs over the Antarctic Peninsula in a chemistry- climate model, Atmos. Chem. Phys., 15 (2), 1071-1086, doi:10.5194/acp-15-1071-2015.

Orr, A., J. S. Hosking, A. Delon, L. Hoffmann, R. Spang, T. Moffat-Griffin, J. Keeble, N. L. Abraham, and P. Braesicke (2020), PSCs initiated by mountain waves in a global chemistry-climate model: A missing piece in fully modelling polar stratospheric ozone depletion, Atmos. Chem. Phys., 20, 1-15, https://doi.org/10.5194/acp-20-1-2020.

Pagan, K. L., A. Tabazadeh, K. Drdla, M. E. Hervig, S. D. Eckermann, E. V. Browell, M. J. Legg, and P. G. Foschi (2004), Observational evidence against mountain-wave generation of ice nuclei as a prerequisite for the formation of three solid nitric acid polar

This article is protected by copyright. All rights reserved. 
stratospheric clouds observed in the arctic in early December 1999, J. Geophys. Res., 109 (D4), doi:10.1029/2003JD003846.

Pawson, S., B. Naujokat, and K. Labitzke (1995), On the polar stratospheric cloud formation potential of the Northern stratosphere, J. Geophys. Res., 100, 23,215-23,225, doi: 10.1029/95JD01918.

Peter, T. (1997), Microphysics and heterogeneous chemistry of polar stratospheric clouds, Ann. Rev. Phys. Chem., 48, 785-822, doi: 10.1146/annurev.physchem.48.1.785.

Peter, T., and J.-U. Grooß (2012), Polar stratospheric clouds and sulfate aerosol particles: Microphysics, denitrification and heterogeneous chemistry, in Stratospheric Ozone

This article is protected by copyright. All rights reserved. 
Depletion and Climate Change, edited by R. Müller, pp. 108-144, Royal Society of Chemistry, doi:10.1039/9781849733182-00108, ISBN: 978-1-84973-002-0.

Peter, T., R. Müller, K. Drdla, K. Petzoldt, E. Reimer (1992). A micro-physical box model for EASOE- preliminary results. Ber. Bunsenges. Phys. Chem., 96, No. 3, 362-367, doi: 10.1002/bbpc. 19920960324.

Peter, I., R. Müller, P. J. Crutzen, and T. Deshler (1994), The lifetime of leewave-induced ice particles in the Arctic stratosphere: II. Stabilization due to NAT-coating, Geophys. Res. Lett., 21, No. 13, 1331-1334, doi: 10.1029/93GL03019.

Pitts, M. C., L. W. Thomason, L. R. Poole, and D. M. Winker (2007), Characterization of Polar Stratospheric Clouds with spaceborne lidar: CALIPSO and the 2006 Antarctic season, Atmos. Chem. Phys., 7 (19), 5207-5228, doi: 10.5194/acp-7-5207-2007.

Pitts, M. C., L. R. Poole, and L. W. Thomason (2009), CALIPSO polar stratospheric cloud observations: second-generation detection algorithm and composition discrimination, Atmos. Chem. Phys., 9 (19), 7577-7589, doi: 10.5194/acp-9-7577-2009.

Pitts, M. C., L. R. Poole, A. Dörnbrack, and L. W. Thomason (2011), The 2009- 2010 Arctic polar stratospheric cloud season: A CALIPSO perspective, Atmos. Chem. Phys., 11 (5), 2161-2177, doi:10.5194/acp-11-2161-2011.

Pitts, M. C., L. R. Poole, A. Lambert, and L. W. Thomason (2013), An assessment of CALIOP polar stratospheric cloud composition classification, Atmos. Chem. Phys., 13 (6), 29752988, doi:10.5194/acp-13-2975-2013.

Pitts, M. C., L. R. Poole, and R. Gonzalez (2018), Polar stratospheric cloud climatology based on CALIPSO spaceborne lidar measurements from 2006 to 2017, Atmos. Chem. Phys., 18 (15), 10,881-10,913, doi:10.5194/acp-18-10881-2018.

Plane, J. M. C. (2012), Cosmic dust in the earth's atmosphere, Chem. Soc. Rev., 41, 6507-6518, doi:10.1039/C2CS35132C.

Poole, L. R., and M. P. McCormick (1988), Polar stratospheric clouds and the Antarctic ozone hole, J. Geophys. Res., 93, 8423-8430, doi: 10.1029/JD093iD07p08423.

Poole, L. R., and M. C. Pitts (1994), Polar stratospheric cloud climatology based on Stratospheric Aerosol Measurement II observations from 1978 to 1989, J. Geophys. Res., 99, 13,083-13,089, doi: 10.1029/94JD00411.

Poole, L. R., M. T. Osborn, and W. H. Hunt (1988), Lidar observations of Arctic polar stratospheric clouds, 1988: Signature of small, solid particles above the frost point, Geophys. Res. Lett., 15 (8), 867-870, doi:10.1029/GL015i008p00867.

Poole, L. R., C. R. Trepte, V. L. Harvey, G. C. Toon, and R. L. VanValkenburg (2003), SAGE III observations of Arctic polar stratospheric clouds - December 2002, Geophys. Res. Lett., 30(23), 2216, doi:10.1029/2003GL018496.

Portmann, R. W., S. Solomon, R. R. Garcia, L. W. Thomason, L. R. Poole, and M.P. McCormick (1996), Role of aerosol variations in anthropogenic ozone depletion in the polar regions, J. Geophys. Res., 101, 22,991-23,006, doi: 10.1029/96JD02608.

This article is protected by copyright. All rights reserved. 
Prata, F., A. Robock, and R. Hamblyn (2018), The Sky in Edvard Munch's The Scream, Bull. Am. Meteorol. Soc., 99 (7), 1377-1390, doi:10.1175/BAMS-D-17-0144.1.

Pruppacher, H., and J. D. Klett (1997), Microphysics of Clouds and Precipitation, 2nd edition, 954 pp., Kluwer Academic Publishers, Dordrecht, Boston, London.

Ravishankara, A. R., and D. R. Hanson (1996), Differences in the reactivity of Type I polar stratospheric clouds depending on their phase, J. Geophys. Res., 101 (D2), 3885-3890, doi: 10.1029/95JD03009.

Read, W. G., A. Lambert, J. Bacmeister, R. E. Cofield, L. E. Christensen, D. T. Cuddy, W. H. Daffer, B. J. Drouin, E. Fetzer, L. Froidevaux, R. Fuller, R. Herman, R. F. Jarnot, J. H. Jiang, Y. Jiang, K. Kelly, B. W. Knosp, L. J.Kovalenko, N. J. Livesey, H.-C. Liu, G. L. Manney, H. M. Pickett, H. C. Pumphrey, K. H. Rosenlof, X. Sabounchi, M. L. Santee, M. J. Schwartz, W. V. Snyder, P. Stek, H. Su, L. L. Takacs, R. P. Thurstans, H. Voemel, P. A. Wagner, J. W. Waters, C. R. Webster, E. M. Weinstock, and D. L. Wu (2007), Aura Microwave Limb Sounder upper tropospheric and lower stratospheric $\mathrm{H}_{2} \mathrm{O}$ and relative humidity with respect to ice validation, J. Geophys. Res., 112 (D24), D24S35, doi: 10.1029/2007JD008752.

Reichardt, J., A. Dörnbrack, S. Reichardt, P. Yang, and T. J. McGee (2004), Mountain wave PSC dynamics and microphysics from ground-based lidar measurements and meteorological modeling, Atmos. Chem. Phys., 4, 1149-1165, doi:10.5194/acp-4-11492004.

Reusch, H. H., (1882), Regnbuefarvede eller iriserende skyer, Naturen, 1, 15.

Rex, M., N. R. P. Harris, P. von der Gathen, R. Lehmann, G. O. Braathen, E. Reimer, A. Beck, M. Chipperfield, R. Alfier, M. Allaart, F. O’Connor, H. Dier, V. Dorokhov, H. Fast, M. Gil, E. Kyrö, Z. Litynska, I. S. Mikkelsen, M. Molyneux, H. Nakane, J. Notholt, M. Rummukainen, P. Viatte, and J. Wenger (1997), Prolonged stratospheric ozone loss in the 1995/96 Arctic winter, Nature, 389, 835-838, doi:10.1038/39849.

Rex, M., R.J. Salawitch, N.R.P. Harris, P. von der Gathen, G.O. Braathen, A. Schulz, H. Deckelmann, M. Chipperfield, B.-M. Sinnhuber, E. Reimer, R. Alfier, R. Bevilacqua, K. Hoppel, M. Fromm, J. Lumpe, H. Küllmann, A. Kleinböhl, H. Bremer, M. von König, K. Künzi, D. Toohey, H. Vömel, E. Richard, K. Aikin, H. Jost, J.B. Greenblatt, M. Loewenstein, J.R. Podolske, C.R. Webster, G.J. Flesch, D.C. Scott, R.L. Herman, J.W. Elkins, E.A. Ray, F.L. Moore, D.F. Hurst, P. Romashkin, G.C. Toon, B. Sen, J.J. Margitan, P. Wennberg, R. Neuber, M. Allart, B.R. Bojkov, H. Claude, J. Davies, W. Davies, H. De Backer, H. Dier, V. Dorokhov, H. Fast, Y. Kondo, E. Kyrö, Z. Litynska, I.S. Mikkelsen, M.J. Molyneux, E. Moran, T. Nagai, H. Nakane, C. Parrondo, F. Ravegnani, P. Skrivankova, P. Viatte, and V. Yushkov (2002), Chemical depletion of

This article is protected by copyright. All rights reserved. 
Arctic ozone in winter 1999/2000, J. Geophys. Res., 107 (D20), doi:10.1029/2001JD000533.

Riviere, E. D., Y. Terao, and H. Nakajima (2003), A Lagrangian method to study stratospheric nitric acid variations in the polar regions as measured by the Improved Limb Atmospheric Spectrometer, J. Geophys. Res. A., 108 (D23), doi: 10.1029/2003JD003718.

Rogers, R. R., \& Yau, M. K. (1989). A short course in cloud physics. Oxford: Pergamon Press.

Rosen, J. M., D. J. Hofmann, J. R. Carpenter, J. W. Harder, and S. J. Oltmans (1988), Balloon borne Antarctic frost point measurements and their impact on polar stratospheric cloud theories, Geophys. Res. Lett., 15 (8), 859-862, doi: 10.1029/GL015i008p00859.

Russell, F. A. R. (1883), Unusual cloud-glow after sunset, Nature, 29 (55), doi: 10.1038/029055a0.

Salawitch, R. J., S. C. Wofsy, and M. B. McElroy (1988), Influence of polar stratospheric clouds on the depletion of Antarctic ozone, Geophys. Res. Lett., 15, 871- 874, doi: 10.1029/GL015i008p00871.

Salawitch, R. J., G. P. Gobbi, S. C. Wofsy, and M. B. McElroy (1989), Denitrification in the Antarctic stratosphere, Nature, 339, 525-527, doi: 10.1038/339525a0.

Santee, M. L., W. G. Read, J. W. Waters, L. Froidevaux, G. L. Manney, D. A. Flower, R. F. Jarnot, R. S. Harwood, and G. E. Peckham (1995), Interhemispheric differences in polar stratospheric $\mathrm{HNO}_{3}, \mathrm{H}_{2} \mathrm{O}, \mathrm{ClO}$, and $\mathrm{O}_{3}$, Science, 267 (5199), 849-852, doi:10.1126/science.267.5199.849.

Santee, M. L., A. Lambert, W. G. Read, N. J. Livesey, R. E. Cofield, D. T. Cuddy, W. H. Daffer, B. J. Drouin, L. Froidevaux, R. A. Fuller, R. F. Jarnot, B. W. Knosp, G. L. Manney, V. S. Perun, W. V. Snyder, P. C. Stek, R. P. Thurstans, P. A. Wagner, J. W. Waters, G. Muscari, R. L. de Zafra, J. E. Dibb, D. W. Fahey, P. J. Popp, T. P. Marcy, K. W. Jucks, G. C. Toon, R. A. Stachnik, P. F. Bernath, C. D. Boone, K. A. Walker, J. Urban, and D. Murtagh (2007), Validation of the Aura Microwave Limb Sounder $\mathrm{HNO}_{3}$ measurements, J. Geophys. Res. A, 112 (D24), doi:10.1029/2007JD008721.

Santee, M. L., G. L. Manney, N. J. Livesey, L. Froidevaux, M. J. Schwartz, and W. G. Read (2011), Trace gas evolution in the lowermost stratosphere from Aura Microwave Limb Sounder measurements, J. Geophys. Res., 116, D18306, doi:10.1029/2011JD015590.

Schneider, J., R. Weigel, T. Klimach, A. Dragoneas, O. Appel, A. Hünig, S. Molleker, F. Köllner, H.-C. Clemen, O. Eppers, P. Hoppe, P. Hoor, C. Mahnke, M. Krämer, C. Rolf, J.-U. Grooß, A. Zahn, F. Obersteiner, F. Ravegnani, A. Ulanovsky, H. Schlager, M. Scheibe, G. S. Diskin, J. P. DiGangi, J. B. Nowak, M. Zöger, and S. Borrmann (2021), Aircraft-based observation of meteoric material in lower-stratospheric aerosol particles between 15 and $68^{\circ}$ N, Atmos. Chem. Phys., 21, 989-1013, https://doi.org/10.5194/acp21-989-2021.

Schröter, J., Rieger, D., Stassen, C., Vogel, H., Weimer, M., Werchner, S., Förstner, J., Prill, F., Reinert, D., Zängl, G., Giorgetta, M., Ruhnke, R., Vogel, B., and Braesicke, P. (2018), ICON-ART 2.1: a flexible tracer framework and its application for composition studies in

This article is protected by copyright. All rights reserved. 
numerical weather forecasting and climate simulations, Geosci. Model Dev., 11, 40434068, doi: 10.5194/gmd-11-4043-2018.

Schütze, K., J. C. Wilson, S. Weinbruch, N. Benker, M. Ebert, G. Günther, R. Weigel, and S. Borrmann (2017), Sub-micrometer refractory carbonaceous particles in the polar stratosphere, Atmos. Chem. Phys., 17 (20), 12,475-12,493, doi:10.5194/acp-17-124752017.

Shi, Q., J. T. Jayne, C. E. Kolb, D. R. Worsnop, and P. Davidovits (2001), Kinetic model for reaction of $\mathrm{ClONO}_{2}$ with $\mathrm{H}_{2} \mathrm{O}$ and $\mathrm{HCl}$ and $\mathrm{HOCl}$ with $\mathrm{HCl}$ in sulfuric acid solutions, $J$. Geophys. Res., 106, 24,259-24,274, doi:10.1029/2000JD000181.

Shibata, T., K. Sato, H. Kobayashi, M. Yabuki, and M. Shiobara (2003), Antarctic polar stratospheric clouds under temperature perturbation by nonorographic inertia gravity waves observed by micropulse lidar at Syowa Station, J. Geophys. Res., 108 (D3), 4105, doi:10.1029/2002JD002713.

Snels, M., A. Scoccione, L. Di Liberto, F. Colao, M. Pitts, L. Poole, T. Deshler, F.Cairo, C. Cagnazzo, and F. Fierli (2019), Comparison of Antarctic polar stratospheric cloud observations by ground-based and space-borne lidar and relevance for chemistry-climate models, Atmos. Chem. Phys., 19, 955-972, doi:10.5194/acp- 19-955-2019.

Snels, M., F. Colao, F. Cairo, I. Shuli, A. Scoccione, M. De Muro, M. Pitts, L. Poole, and L. Di Liberto (2021), Quasi-coincident observations of polar stratospheric clouds by ground-

This article is protected by copyright. All rights reserved. 
based lidar and CALIOP at Concordia (Dome C, Antarctica) from 2014 to 2018, Atmos. Chem. Phys., 21, 2165-2178, doi.org/10.5194/acp-21-2165-2021.

Solomon, P. M., B. Connor, R. L. de Zafra, A. Parrish, J. Barrett, and J. Jaramillo (1987a), High concentrations of chlorine monoxide at low altitudes in the Antarctic spring stratosphere: secular variation, Nature, 328, 411-413, https://doi.org/10.1038/328411a0.

Solomon, S. (1988), The mystery of the Antarctic ozone "hole", Rev. Geophys., 26, 131-148.

Solomon, S. (1999), Stratospheric ozone depletion: A Review of concepts and history, Rev. Geophys., 37 (3), 275-316.

Solomon, S., R. R. Garcia, F. S. Rowland, and D. J. Wuebbles (1986), On the depletion of Antarctic ozone, Nature, 321, 755-758, doi: 10.1029/RG026i001p00131.

Solomon, S., H. G. Mount, R. W. Sanders, and A. L. Schmeltekopf (1987b), Visible spectroscopy at McMurdo station, Antarctica 2. Observations of OClO, J. Geophys. Res., 92, 8329-8338, doi: 10.1029/JD092iD07p08329.

Solomon, S., D. Kinnison, J. Bandoro, and R. Garcia (2015), Simulation of polar ozone depletion: An update, J. Geophys. Res., 120, 7958-7974, doi: 10.1002/2015JD023365.

Spang, R., M. Riese, and D. Offermann (2001), CRISTA-2 observations of the South Polar Vortex in winter 1997: A new dataset for polar process studies, Geophys. Res. Lett., 28, 3159-3162, doi: 10.1029/2000GL012374

Spang, R., and J. Remedios (2003), Observations of a distinctive infra-red spectral feature in the atmospheric spectra of polar stratospheric clouds measured by the CRISTA instrument, Geophys. Res. Lett., 30, 1875, doi:10.1029/2003GL017231.

Spang, R., J. J. Remedios, L. J. Kramer, L. R. Poole, M. D. Fromm, M. Müller, G. Baumgarten, and P. Konopka (2005), Polar stratospheric cloud observations by MIPAS on ENVISAT: Detection method, validation and analysis of the Northern hemisphere winter 2002/2003, Atmos. Chem. Phys., 5, 679-692, doi: 10.5194/acp-5-679-2005.

Spang, R., K. Arndt, A. Dudhia, M. Höpfner, L. Hoffmann, J. Hurley, R. G. Grainger, S. Griessbach, C. Poulsen, J. J. Remedios, M. Riese, H. Sembhi, R. Siddans, A. Waterfall, and C. Zehner (2012), Fast cloud parameter retrievals of MIPAS/Envisat, Atmos. Chem. Phys., 12 (15), 7135-7164, doi:10.5194/acp-12-7135-2012.

Spang, R., L. Hoffmann, M. Höpfner, S. Griessbach, R. Müller, M. C. Pitts, A. M. W. Orr, and M. Riese (2016), A multi-wavelength classification method for polar stratospheric cloud types using infrared limb spectra, Atmos. Meas. Tech., 9 (8), 3619-3639, doi:10.5194/amt-9-3619-2016.

Spang, R., L. Hoffmann, R. Müller, J.-U. Grooß, I. Tritscher, M. Höpfner, M. Pitts, A. Orr, and M. Riese (2018), A climatology of polar stratospheric cloud composition between 2002

This article is protected by copyright. All rights reserved. 
and 2012 based on MIPAS/Envisat observations, Atmos. Chem. Phys., 18 (7), 50895113, doi:10.5194/acp-18-5089-2018.

Stanford, J.L, and J. S. Davis (1974) A century of stratospheric cloud reports: 1870-1972, Bull. Am. Met. Soc., doi: 10.1175/1520-0477(1974)055\%3C0213:ACOSCR\%3E2.0.CO;2.

Stefanutti, L., et al. (Eds.) (1999), The Airborne Platform of Earth observations (APE): The contribution to THESEO, vol. Contract-No. ENV4-CT97-0533 [DG- 12-EHKN], environmental and climate research programme, Final Report.

Steiner, M., Luo, B., Peter, T., Pitts, M. C., and Stenke, A. (2021), Evaluation of polar stratospheric clouds in the global chemistry-climate model SOCOLv3.1 by comparison with CALIPSO spaceborne lidar measurements, Geosci. Model Dev., 14, 935-959, https://doi.org/10.5194/gmd-14-935-2021.

Stenke, A., M. Schraner, E. Rozanov, T. Egorova, B. Luo, and T. Peter (2013), The SOCOL version 3.0 chemistry-climate model: Description, evaluation, and implications from an advanced transport algorithm, Geosci. Model Dev., 6, 1407-1427, doi:10.5194/gmd-61407-2013.

Stephens, G. L., D. G. Vane, R. J. Boain, G. G. Mace, K. Sassen, Z. Wang, A. J. Illingworth, E. J. O' connor, W. B. Rossow, S. L. Durden, S. D. Miller, R. T. Austin, A. Benedetti, and C. a. Mitrescu (2002), The CloudSat mission and the a-train, Bull. Am. Meteorol. Soc., 83 (12), 1771-1790, doi:10.1175/BAMS-83-12-1771.

Stetzer, O., O. Möhler, R. Wagner, S. Benz, H. Saathoff, H. Bunz, and O. Indris (2006), Homogeneous nucleation rates of nitric acid dihydrate (NAD) at simulated stratospheric

This article is protected by copyright. All rights reserved. 
conditions - Part I: Experimental results, Atmos. Chem. Phys., 6 (10), 3023-3033, doi:10.5194/acp-6-3023-2006.

Størmer, C. (1929), Remarkable clouds at high altitudes, Nature, 123 (3094), 260-261, doi:10.1038/123260a0.

Størmer, C. (1932a), Höhenmessungen von Stratosphärenwolken, Beitr. Phys. Atmos., 21, 1-6.

Størmer, C. (1932b), Mother-of-Pearl Clouds over Scandinavia in January and February 1932, Nature, 129, 941.

Størmer, C. (1935), Luminous Night Clouds over Norway in 1933 and 1934, Nature, 135, 103104, https://doi.org/10.1038/135103b0

Størmer, C. (1948), Weather, 3 (13).

Strawa, A. W., K. Drdla, M. Fromm, R. F. Pueschel, K. W. Hoppel, E. V. Browell, P. Hamill, and D. P. Dempsey (2002), Discriminating Types Ia and Ib polar stratospheric clouds in POAM satellite data, J. Geophys. Res., 107(D20), 8291, doi:10.1029/2001JD000458.

Tabazadeh, A., R. P. Turco, K. Drdla, M. Z. Jacobson, and O. B. Toon (1994), A study of type I polar stratospheric cloud formation, Geophys. Res. Lett., 21, 1619-1622, doi: 10.1029/94GL01368.

Tabazadeh, A., O. B. Toon, and P. Hamill (1995), Freezing behavior of stratospheric sulfate aerosols inferred from trajectory studies, Geophys. Res. Lett., 22 (13), 1725-1728, doi:10.1029/95GL01335.

Tabazadeh, A., Y. S. Djikaev, P. Hamill, and H. Reiss (2002), Laboratory evidence for surface nucleation of solid Polar Stratospheric Cloud particles, J. Phys. Chem. A, 106 (5), $10,238-10,246$.

Taylor, F. W, A. Lambert, R. G. Grainger, C. D. Rodgers, and J. J. Remedios (1994), Properties of Northern Hemisphere polar stratospheric clouds and volcanic aerosol in 1991/92 from UARS/ISAMS satellite measurements, J. Atmos. Sci., 51, 3019-3026, 1994.

Teisserenc de Bort, L. (1902), Variations de la temperature de l'air libre dans la zone comprise 8 km et 13 km d'altitude, C. R. Hebd. Seance Acad. Sci., 24, 987-989.

Teitelbaum, H., and R. Sadourny (1998), The role of planetary waves in the formation of polar stratospheric clouds, Tellus A: Dynamic Meteorology and Oceanography, 50 (3), 302312, doi:10.3402/tellusa.v50i3.14528.

Teitelbaum, H., M. Moustaoui, and M. Fromm (2001), Exploring polar stratospheric cloud and ozone minihole formation: The primary importance of synoptic-scale flow perturbations, J. Geophys. Res., 106, 28,173-28,188, doi: 10.1029/2000JD000065.

Thomason, L., and T. Peter (Eds.) (2006), SPARC Assessment of Stratospheric Aerosol Properties, SPARC Report No. 4, WCRP-124, WMO/TD-No.1295.

Tizek, H., E. Knözinger, and H. Grothe (2002), X-ray diffraction studies on nitric acid dihydrate, Phys. Chem. Chem. Phys., 4, 5128-5134, doi:10.1039/B206644K.

Tizek, H., E. Knözinger, and H. Grothe (2004), Formation and phase distribution of nitric acid hydrates in the mole fraction range $\mathrm{x} \mathrm{HNO}_{3}<0.25$ : A combined XRD and IR study, Phys. Chem. Chem. Phys., 6, 972-979, doi:10.1039/B310672A.

This article is protected by copyright. All rights reserved. 
Tolbert, M. A., M. J. Rossi, R. Malhotra, and D. M. Golden (1987), Reaction of chlorine nitrate with hydrogen-chloride and water at Antarctic stratospheric temperatures, Science, 238 (4831), 1258-1260, doi:10.1126/science.238.4831.1258.

Toon, O. B., and N. H. Farlow (1981), Particles Above the Tropopause: Measurements and Models of Stratospheric Aerosols, Meteoric Debris, Nacreous Clouds, and Noctilucent Clouds, Ann. Rev. Earth Planet. Sci., 9 (1), 19-58, doi: 10.1146/annurev.ea.09.050181.000315.

Toon, O. B., and M. A. Tolbert (1995), Spectroscopic evidence against nitric acid trihydrate in polar stratospheric clouds, Nature, 375, 218-221, doi: 10.1038/375218a0.

Toon, O. B., P. Hamill, R. P. Turco, and J. Pinto (1986), Condensation of $\mathrm{HNO}_{3}$ and $\mathrm{HCl}$ in winter polar stratospheres, Geophys. Res. Lett., 13, 1284-1287, doi: 10.1029/GL013i012p01284.

Toon, G. C., Farmer, C. B., Lowes, L. L., Schaper, P. W., Blavier, J.-F., and Norton, R. H. (1989a), Infrared aircraft measurements of stratospheric composition over Antarctica during September 1987, J. Geophys. Res., 94, 16571-16596, doi: 10.1029/JD094iD14p16571.

Toon, O. B., R. P. Turco, J. Jordan, J. Goodman, and G. Ferry (1989b), Physical processes in polar stratospheric ice clouds, J. Geophys. Res., D9, 11,359-11,380, doi: 10.1029/JD094iD09p11359.

Toon, O. B., E. V. Browell, S. Kinne, and J. Jordan (1990), An analysis of LIDAR observations of polar stratospheric clouds, Geophys. Res. Lett., 17, 393-396, doi: 10.1029/GL017i004p00393.

Tritscher, I., J.-U. Grooß, R. Spang, M. P. Pitts, L. R. Poole, R. Müller, and M. Riese (2019), Lagrangian simulation of ice particles and resulting dehydration in the polar winter stratosphere, Atmos. Chem. Phys., 19, 543-563, doi: 10.5194/acp-19-543-2019.

Tsias, A., M. Wirth, K. S. Carslaw, J. Biele, H. Mehrtens, J. Reichardt, C. Wedekind, V. Weiss, W. Renger, R. Neuber, U. von Zahn, B. Stein, V. Santacesaria, L. Stefanutti, F. Fierli, J. Bacmeister, and T. Peter (1999), Aircraft lidar observations of an enhanced type Ia polar stratospheric clouds during APE- POLECAT, J. Geophys. Res., 104 (D19), 23,96123,969, doi: 10.1029/1998JD100055.

Tuck, A. F., R. T. Watson, E. P. Condon, J. J. Margitan, and O. B. Toon (1989), The planning and execution of ER-2 and DC-8 aircraft flights over Antarctica, August and September 1987, J. Geophys. Res., 94, 11,181-11,222, doi: 10.1029/JD094iD09p11181.

Turco, R. P., O. B. Toon, and P. Hamill (1989), Heterogeneous physicochemistry of the polar ozone hole, J. Geophys. Res., 94 (D14), 16,493-16,510, doi: 10.1029/89JD00998.

Turco, R. P., A. Plumb, and E. Condon (1990), The airborne Arctic stratospheric expedition: Prologue, Geophys. Res. Lett., 17 (4), 313-316, doi: 10.1029/GL017i004p00313/abstract.

Voigt, C., J. Schreiner, A. Kohlmann, P. Zink, K. Mauersberger, N. Larsen, T. Deshler, C. Kröger, J. Rosen, A. Adriani, F. Cairo, G. D. Donfrancesco, M. Viterbini, J. Ovarlez, H.

This article is protected by copyright. All rights reserved. 
Ovarlez, C. David, and A. Dörnbrack (2000), Nitric acid trihydrate (NAT) in polar stratospheric clouds, Science, 290, 1756-1758, doi: 10.1126/science.290.5497.1756.

Voigt, C., N. Larsen, T. Deshler, C. Kröger, J. Schreiner, K. Mauersberger, B. P. Luo, A. Adriani, F. Cairo, G. D. Donfrancesco, J. Ovarlez, H. Ovarlez, A. Dörnbrack, B. Knudsen, and J. Rosen, In situ mountain-wave polar stratospheric cloud measurements: Implications for nitric acid trihydrate formation (2003), J. Geophys. Res., 108 (D5), 8331, doi:10.1029/2001JD001185.

Voigt, C., H. Schlager, B. Luo, A. Dörnbrack, A. Roiger, P. Stock, J. Curtius, H. Vössing, S. Borrmann, S. Davies, P. Konopka, C. Schiller, G. Shur, and T. Peter (2005), Nitric acid trihydrate (NAT) formation at low NAT supersaturation in polar stratospheric clouds (PSCs), Atmos. Chem. Phys., 5, 1371-1380, doi: /10.5194/acp-5-1371-2005.

Voigt, C., A. Dörnbrack, M. Wirth, S. M. Groß, M. C. Pitts, L. R. Poole, R. Baumann, B. Ehard, B.-M. Sinnhuber, W. Woiwode, and H. Oelhaf (2018), Widespread polar stratospheric ice clouds in the 2015-2016 Arctic winter - implications for ice nucleation, Atmos. Chem. Phys., 18 (21), 15,623-15,641, doi: 10.5194/acp-18-15623-2018.

Volkert, H., and D. Intes (1992), Orographically forced stratospheric waves over northern Scandinavia, Geophys. Res. Lett., 19, 1205-1208, doi: 10.1029/92GL01160.

von Clarmann, T., Höpfner, M., Kellmann, S., Linden, A., Chauhan, S., Funke, B., Grabowski, U., Glatthor, N., Kiefer, M., Schieferdecker, T., Stiller, G. P., and Versick, S. (2009), Retrieval of temperature, $\mathrm{H}_{2} \mathrm{O}, \mathrm{O}_{3}, \mathrm{HNO}_{3}, \mathrm{CH}_{4}, \mathrm{~N}_{2} \mathrm{O}, \mathrm{ClONO}_{2}$ and $\mathrm{ClO}$ from MIPAS reduced resolution nominal mode limb emission measurements, Atmos. Meas. Tech., 2, 159-175, doi:10.5194/amt-2-159-2009.

von Hobe, M., S. Bekki, S. Borrmann, F. Cairo, F. D'Amato, G. Di Donfrancesco, A. Dörnbrack, A. Ebersoldt, M. Ebert, C. Emde, I. Engel, M. Ern, W. Frey, S. Genco, S. Griessbach, J.U. Grooß, T. Gulde, G. Günther, E. Hösen, L. Hoffmann, V. Homonnai, C. R. Hoyle, I. S. A. Isaksen, D. R. Jackson, I. M. J'anosi, R. L. Jones, K. Kandler, C. Kalicinsky, A. Keil, S. M. Khaykin, F. Khosrawi, R. Kivi, J. Kuttippurath, J. C. Laube, F. Lef evre, R. Lehmann, S. Ludmann, B. P. Luo, M. Marchand, J. Meyer, V. Mitev, S. Molleker, R. Müller, H. Oelhaf, F. Olschewski, Y. Orsolini, T. Peter, K. Pfeilsticker, C. Piesch, M. C. Pitts, L. R. Poole, F. D. Pope, F. Ravegnani, M. Rex, M. Riese, T. Röckmann, B. Rognerud, A. Roiger, C. Rolf, M. L. Santee, M. Scheibe, C. Schiller, H. Schlager, M. Siciliani de Cumis, N. Sitnikov, O. A. Søvde, R. Spang, N. Spelten, F. Stordal, O. Sumińska-Ebersoldt, A. Ulanovski, J. Ungermann, S. Viciani, C. M. Volk, M. vom Scheidt, P. von der Gathen, K. Walker, T. Wegner, R. Weigel, S. Weinbruch, G. Wetzel, F. G. Wienhold, I. Wohltmann, W. Woiwode, I. A. K. Young, V. Yushkov, B. Zobrist, and F. Stroh (2013), Reconciliation of essential process parameters for an enhanced predictability of Arctic stratospheric ozone loss and its climate interactions

This article is protected by copyright. All rights reserved. 
(RECONCILE): Activities and results, Atmos. Chem. Phys., 13 (18), 9233-9268, doi:10.5194/acp-13-9233-2013.

Waibel, A. E., T. Peter, K. S. Carslaw, H. Oelhaf, G. Wetzel, P. J. Crutzen, U. Pöschl, A. Tsias, E. Reimer, and H. Fischer (1999), Arctic ozone loss due to denitrification, Science, 283, 2064-2069, doi: 10.1126/science.283.5410.2064.

Wang, Z., G. Stephens, T. Deshler, C. Trepte, T. Parish, D. Vane, D. Winker, D. Liu, and L. Adhikari (2008), Association of Antarctic polar stratospheric cloud formation on tropospheric cloud systems, Geophys. Res. Lett., 35 (13), doi:10.1029/2008GL034209.

Ward, S. M., T. Deshler, and A. Hertzog (2014), Quasilagrangian measurements of nitric acid trihydrate formation over Antarctica, J. Geophys. Res., 119 (1), 245-258, doi:10.1002/2013JD020326.

Waters, J. W., L. Froidevaux, R. S. Harwood, R. F. Jarnot, H. M. Pickett, W. G. Read, P. H. Siegel, R. E. Cofield, M. J. Filipiak, D. A. Flower, J. R. Holden, G. K. Lau, N. J. Livesey, G. L. Manney, H. C. Pumphrey, M. L. Santee, D. L. Wu, D. T. Cuddy, R. R. Lay, M. S. Loo, V. S. Perun, M. J. Schwartz, P. C. Stek, R. P. Thurstans, M. A. Boyles, S. Chandra, M. C. Chavez, G.-S. Chen, B. V. Chudasama, R. Dodge, R. A. Fuller, M. A. Girard, J. H. Jiang, Y. Jiang, B. W. Knosp, R. C. LaBelle, J. C. Lam, K. A. Lee, D. Miller, J. E. Oswald, N. C. Patel, D. M. Pukala, O. Quintero, D. M. Scaff, W. V. Snyder, M. C. Tope, P. A. Wagner, and M. J. Walch (2006), The Earth Observing System Microwave Limb Sounder (EOS MLS) on the Aura satellite, IEEE Trans. Geosci. Remote Sens., 44 (5), 1106-1121, doi: 10.1109/TGRS.2006.873771.

Wegner, T., J.-U. Grooß, M. von Hobe, F. Stroh, O. Sumińska-Ebersoldt, C. M. Volk, E. Hösen, V. Mitev, G. Shur, and R. Müller (2012), Heterogeneous chlorine activation on stratospheric aerosols and clouds in the arctic polar vortex, Atmos. Chem. Phys., 12, 11,095-11,106, doi:10.5194/acp-12-11095-2012.

Wegner, T., D. E. Kinnison, R. R. Garcia, and S. Solomon (2013), Simulation of polar stratospheric clouds in the specified dynamics version of the Whole Atmosphere Community Climate Model, J. Geophys. Res., pp. 4991-5002, doi: 10.1002/jgrd.50415.

Wegner, T., M. C. Pitts, L. R. Poole, I. Tritscher, J.-U. Grooß, and H. Nakajima (2016), Vortexwide chlorine activation by a mesoscale PSC event in the Arctic winter of 2009/10, Atmos. Chem. Phys., 16, 4569-4577, doi:10.5194/acp-16-4569-2016.

Weigel, R., C. M. Volk, K. Kandler, E. Hösen, G. Günther, B. Vogel, J.-U. Grooß, S. Khaykin, G. V. Belyaev, and S. Borrmann (2014), Enhancements of the refractory submicron

This article is protected by copyright. All rights reserved. 
aerosol fraction in the Arctic polar vortex: feature or exception?, Atmospheric Chemistry and Physics, 14 (22), 12,319-12,342, doi:10.5194/acp-14- 12319-2014.

Wilmouth, D. M., R. M. Stimpfle, J. G. Anderson, J. W. Elkins, D. F. Hurst, R. J. Salawitch, and L. R. Lait (2006), Evolution of inorganic chlorine partitioning in the Arctic polar vortex, J. Geophys. Res., 111, D16308, doi:10.1029/2005JD006951.

Wilmouth, D. M., R. J. Salawitch, and T. P. Canty (2018), Stratospheric ozone depletion and recovery, in Green Chemistry, edited by B. Török and T. Dransfield, pp. 177-209, Elsevier.

Winker, D. M., M. A. Vaughan, A. Omar, Y. Hu, K. A. Powell, Z. Liu, W. H. Hunt, and S. A. Young (2009), Overview of the CALIPSO Mission and CALIOP Data Processing Algorithms, J. Atmos. Ocean. Technol., 26 (11), 2310-2323, doi: 10.1175/2009JTECHA1281.1.

Wirth, M., A. Tsias, A. Dörnbrack, V. Weiss, K. Carslaw, M. Leutbecher, W. Renger, H. Volkert, and T. Peter (1999), Model-guided Lagrangian observation and simulation of mountain polar stratospheric clouds, J. Geophys. Res., 104 (D19), 23,971-23,981, doi:10.1029/1998JD100095.

WMO (2018), World Meteorological Organization, Scientific Assessment of Ozone Depletion: 2018, Global Ozone Research and Monitoring Project-Report No. 58, 588 pp., Geneva, Switzerland.

Wohltmann, I., R. Lehmann, and M. Rex (2010), The Lagrangian chemistry and transport model ATLAS: Simulation and validation of stratospheric chemistry and ozone loss in the winter 1999/2000, Geosci. Model Dev., 3 (2), 585-601, doi: 10.5194/gmd-3-585-2010.

Wohltmann, I., R. Lehmann, and M. Rex (2017), A quantitative analysis of the reactions involved in stratospheric ozone depletion in the polar vortex core, Atmos. Chem. Phys., 17 (17), 10,535-10,563, doi:10.5194/acp-17-10535-2017.

Woiwode, W., J.-U. Grooß, H. Oelhaf, S. Molleker, S. Borrmann, A. Ebersoldt, W. Frey, T. Gulde, S. Khaykin, G. Maucher, C. Piesch, and J. Orphal (2014), Denitrification by large NAT particles: The impact of reduced settling velocities and hints on particle characteristics, Atmos. Chem. Phys., 14 (20), 11,525-11,544, doi:10.5194/acp-14-115252014.

Woiwode, W., M. Höpfner, L. Bi, M. C. Pitts, L. R. Poole, H. Oelhaf, S. Molleker, S. Borrmann, M. Klingebiel, G. Belyaev, A. Ebersoldt, S. Griessbach, J.-U. Grooß, T. Gulde, M. Krämer, G. Maucher, C. Piesch, C. Rolf, C. Sartorius, R. Spang, and J. Orphal (2016), Spectroscopic evidence of large aspherical $\beta$-NAT particles involved in denitrification in

This article is protected by copyright. All rights reserved. 
the December 2011 Arctic stratosphere, Atmos. Chem. Phys., 16 (14), 9505-9532, doi:10.5194/acp-16-9505-2016.

Woiwode, W., Höpfner, M., Bi, L., Khosrawi, F., and Santee, M. L. (2019). Vortex-Wide Detection of Large Aspherical NAT Particles in the Arctic Winter 2011/12 Stratosphere, Geophys. Res. Lett., 46, 13420- 13429, doi:10.1029/2019GL084145.

Wooldridge, P. J., R. Zhang, and M. J. Molina (1995), Phase equilibria of $\mathrm{H}_{2} \mathrm{SO}_{4}, \mathrm{HNO}_{3}$, and $\mathrm{HCl}$ hydrates and the composition of polar stratospheric clouds, J. Geophys. Res. A, 100 (D1), 1389-1396, doi:10.1029/94JD02745.

Worsnop, D. R., L. E. Fox, M. S. Zahniser, and S. C. Wofsy (1993), Vapor pressures of solid hydrates of Nitric Acid: Implications for polar stratospheric clouds, Science, 259, 71-74, doi: 10.1126/science.259.5091.71.

Zhang, R., J. T. Jayne, and M. J. Molina (1994), Heterogeneous interactions of $\mathrm{ClONO}_{2}$ and $\mathrm{HCl}$ with sulfuric acid tetrahydrate: Implications for the stratosphere, J Phys Chem, 98, 867874, doi: 10.1002/chin.199419014.

Zhang Y., O. R. Cooper, A. Gaudel, A. M. Thompson, P. Nédélec, S.-Y. Ogino, and J. J. West (2016), Tropospheric ozone change from 1980 to 2010 dominated by equatorward redistribution of emissions, Nature Geoscience, 9, 875-881, doi:http://dx.doi.org/10.1038/ngeo2827 10.1038/ngeo2827.

Zhu, Y., O. B. Toon, A. Lambert, D. E. Kinnison, M. Brakebusch, C. G. Bardeen, M. J. Mills, and J. M. English (2015), Development of a Polar Stratospheric Cloud Model within the Community Earth System Model using constraints on Type I PSCs from the 2010-2011 Arctic winter, J. Advances in Modeling Earth Sys., 7, 551-585, doi:10.1002/2015MS000427.

Zhu, Y., O. B. Toon, M. C. Pitts, A. Lambert, C. Bardeen, and D. E. Kinnison (2017a), Comparing simulated PSC optical properties with CALIPSO observations during the 2010 Antarctic winter, J. Geophys. Res. A, 122, 1175-1202, doi:10.1002/2016JD025191.

Zhu, Y., O. B. Toon, A. Lambert, D. E. Kinnison, C. Bardeen, and M. C. Pitts (2017b), Development of a polar stratospheric cloud model within the Community Earth System Model: Assessment of 2010 Antarctic winter, J. Geophys. Res. A, 122 (19), 10,50310,523, doi:10.1002/2017JD027003.

This article is protected by copyright. All rights reserved. 


\section{Figure Captions}

Figure 1. (a) PSCs photographed by Carl Størmer from Oslo towards the west directly after sunset on 13 January 1929 (Størmer, 1929); (b) Størmer determined cloud altitudes (22.3-25.3 $\mathrm{km}$ ) by simultaneous measurements from Oslo and Oscarborg, referenced to stars in the constellations of Aquila and Delphinus; (c) Photograph of PSCs above Finland in February 2017 (courtesy of Alpo Vuontisjärvi, www.ounasloma.fi); (d) Edvard Munch's famous self-portrait "The Scream" (1893), with the sky screaming at him. Recently it has been hypothesized (Fikke et al., 2017; Prata et al., 2018) that the sky in "The Scream" is strikingly similar to PSCs.

Figure 2. (a) 2-D histogram of CALIOP Antarctic PSC data for 10-18 July 2008 at latitudes 65$75^{\circ} \mathrm{S}$ and potential temperatures $(\theta) 475-525 \mathrm{~K}$. (b) Theoretical optical calculations for nonequilibrium liquid-NAT and liquid-ice mixtures. The dashed and grey boxes at the lower left represent points that fall below both CALIOP v2 PSC detection thresholds and are classified as non-PSCs, i.e. background stratospheric sulfuric acid aerosols (SSA).

Figure 3. Curtains from one CALIPSO orbit across the Antarctic on 17 July 2008 of (a) $R_{532}$; (b) $\beta_{\perp}$; (c) inferred PSC composition; (d) estimated SAD, and (e) map showing location of orbit track. Thick black line in panels (c) and (d) indicate the MERRA-2 tropopause heights.

Figure 4. (a-d) Measured MIPAS spectra illustrating characteristic spectral features of NAT $(<$ 2-3 $\mu \mathrm{m}$ radius), large NAT, ice and STS PSCs; (e-f) Retrieved MIPAS PSC composition category and VD near $21 \mathrm{~km}$ altitude for the same day as the spectra in (a, d). (g) Profiles of PSC particle volume density (VD) retrieved from the spectra in (a). The light blue curve in (e) is the CALIPSO orbit track from Figure 3, and the highlighted triangles in (e) and (f) mark the location of the VD profile in $(\mathrm{g})$.

Figure 5. Polar stereographic projection of the Aura MLS orbits over a region encompassing the Ross Ice Shelf from 23-25 May 2008 showing the evolution of $\mathrm{HNO}_{3}$ on (a) $46 \mathrm{hPa}(\sim 20 \mathrm{~km})$ and (b) $68 \mathrm{hPa}(\sim 18 \mathrm{~km})$ atmospheric levels. The locations of the South Pole and McMurdo are indicated on each panel by square and diamond symbols, respectively. Orbit track filled symbol colors refer to the numerical scale for $\mathrm{HNO}_{3}$ volume mixing rations on the left. Adapted from Lambert et al. (2012).

Figure 6. (a) 17 July 2008 CALIOP PSC composition curtain from Figure 2(c); (b) $T_{\text {MERRA-2 - }}$ $T_{\text {ice }}$ along this orbit track, where $T_{\text {MERRA-2 }}$ is interpolated from MERRA-2 analyses and $T_{\text {ice }}$ is calculated using MLS $\mathrm{H}_{2} \mathrm{O}$ data; (c-d) $\mathrm{MLS} \mathrm{H}_{2} \mathrm{O}$ and $\mathrm{HNO}_{3}$ along the orbit track.

Figure 7. Bottom: Equilibrium fractional uptake of gas phase $\mathrm{HNO}_{3}$ into STS (Carlsaw et al., $1995)$ as a function of $T-T_{\text {ice }}$ and $T$ - $T_{\text {NAT }}$. Top: STS 532-nm backscatter ratio $\left(R_{532}\right.$, purple curve) and STS particle VD (red curve) as a function of $\mathrm{HNO}_{3}$ condensed in STS. Dashed lines indicate STS detection thresholds for CALIOP (purple), MIPAS (red), MLS (blue), and groundbased lidar (black).

Figure 8. NAT detection sensitivity of liquid-NAT mixtures for a range of NAT number densities and effective radii at $T=T_{\text {ice }}+5 \mathrm{~K}$, pressure $=46 \mathrm{hPa}, \mathrm{H}_{2} \mathrm{O}=5 \mathrm{ppmv}$, and total $\mathrm{HNO}_{3}=12$ ppbv. Detection thresholds are shown for MLS (green solid line), MIPAS (dashed red lines), and CALIOP (dashed purple curve - $R_{532}$; dashed blue curve - $\beta_{\perp}$ ). The gray shaded area is not detectable by any of the three instruments. Light blue areas indicate the particle size and number density regimes for enhanced NAT, synoptic NAT, and NAT rocks.

This article is protected by copyright. All rights reserved. 
Figure 9. MIPAS limb-viewing paths through an Antarctic CALIOP PSC orbital curtain on 24 August 2011, assuming both instruments measured in the same orbital plane. Color scale at right indicates CALIOP PSC composition, and color-coded symbols indicate retrieved MIPAS PSC composition.

Figure 10. MIPAS limb-viewing paths through a spatially inhomogeneous Antarctic CALIOP PSC orbital curtain on 29 June 2011, assuming both instruments measured in the same orbital plane. Color scale at right indicates CALIOP PSC composition, and color-coded symbols indicate retrieved MIPAS PSC composition.

Figure 11. PSC areal coverage during the 2009 Antarctic winter observed by CALIOP (left) and MIPAS (right). Top row = all PSCs. Second row = STS (CALIOP) and STSmix (MIPAS). Third row $=$ ice + wave ice $($ CALIOP $)$ and ice $($ MIPAS $)$. Fourth row $=$ NAT mixtures + enhanced NAT mixtures (CALIOP) and NAT (MIPAS).

Figure 12. CALIOP and scaled MIPAS daily PSC volumes for 2002-2018 for (a) Antarctic, May-Sept. and (b) Arctic, Dec.-Mar. Note the different scales in (a) and (b).

Figure 13. Comparison of CALIOP and MIPAS PSC composition for spatially homogeneous coincident scenes in the (a) Antarctic and (b) Arctic from 2006-2011.

Figure 14. Histograms of CALIOP v2 PSC observations by composition at $21 \mathrm{~km}$ altitude from 13 Antarctic and 12 Arctic winters as a function of (a, c) $T-T_{\text {ice }}$ and (b, d) $T-T_{\text {eq }}$, where $T$ is the ambient temperature at CALIOP/MLS observation points interpolated from MERRA-2 reanalyses and $T_{\text {eq }}$ is equal to $T_{\text {ice, }} T_{\mathrm{NAT}}$ or $T_{\mathrm{STS}}$ (depending on the respective composition class) calculated using MLS gas-phase $\mathrm{H}_{2} \mathrm{O}$ and $\mathrm{HNO}_{3}$ data. Green = STS; orange = NAT mixtures + enhanced NAT mixtures; and dark blue = ice + wave ice. Updated from Pitts et al. (2018).

Figure 15. PSC observations in 2006 above McMurdo. (top) CALIOP around McMurdo; (bottom) Ground-based lidar data. Green = STS; yellow = NAT mixtures; red = enhanced NAT mixtures; and blue $=$ ice + wave ice. Triangles on the time axis indicate the days when at least one observation was available. Adapted from Snels et al. (2019).

Figure 16. PSC occurrence frequencies for June-September 2006-2010 from (left) ground-based lidar measurements at McMurdo and (right) CALIOP v2 data within $\pm 1^{\circ}$ latitude and $\pm 3.5^{\circ}$ longitude of McMurdo. Updated from Pitts et al. (2018).

Figure 17. 2006-2018 mean daily CALIOP PSC/cloud areal coverage over the (a) Antarctic and (b) Arctic. The climatological daily maximum MERRA-2 tropopause heights (dashed white lines) indicate the upper extent of cirrus. Updated from Pitts et al. (2018).

Figure 18. Time series of 2006-2018 mean, standard deviation, and range of daily values of CALIOP (a) Antarctic and (b) Arctic PSC spatial volume (daily areal coverage integrated over altitude). Maxima and minima are color-coded by the year in which they occurred. Updated from Pitts et al. (2018).

Figure 19. 2006-2018 Antarctic mean relative spatial coverage for CALIOP (a) STS; (b) NAT mixtures, including enhanced NAT mixtures; and (c) ice, including wave ice. Thick black line: PSCs in at least 6 of the 13 Antarctic seasons. White region in (c): no ice occurrence detected. Panel (d) shows 2006-2018 mean distribution of $T$ - $T_{\text {NAT. }}$. Updated from Pitts et al. (2018).

Figure 20. Vertical profiles of 2006-2018 mean season-long CALIOP relative spatial coverage by PSC composition for the (a) Antarctic and (b) Arctic. Horizontal bars are 1- $\sigma$ uncertainties in

This article is protected by copyright. All rights reserved. 
the multi-year mean values and are offset by $0.1 \mathrm{~km}$ to avoid overlap. Updated from Pitts et al. (2018).

Figure 21. 2006-2018 average, monthly mean polar maps of CALIOP Antarctic PSC occurrence frequency at $\theta=500 \mathrm{~K}(\sim 20 \mathrm{~km})$. (Top row) All PSCs; (row 2) STS; (row 3) NAT mixtures, including enhanced NAT; (bottom row) ice, including wave ice). Black curves show mean vortex edge. Solid red (white) curves enclose region where mean $T<T_{\mathrm{NAT}}\left(T<T_{\text {ice }}\right.$ ). Light gray regions: not sampled by CALIOP. Updated from Pitts et al. (2018).

Figure 22. 2006-2018 average, monthly mean polar maps of CALIOP Arctic PSC occurrence frequency at $\theta=500 \mathrm{~K}(\sim 20 \mathrm{~km})$. Light gray regions: not sampled by CALIOP. Adapted from Pitts et al. (2018).

Figure 23. Equivalent latitude/potential temperature cross sections of 2006-2018 average, monthly zonal mean of (top row) CALIOP Antarctic PSC occurrence frequency, (row 2) cloudfree $\mathrm{MLS} \mathrm{HNO}_{3}$, (row 3) cloud-free MLS H $\mathrm{H}_{2} \mathrm{O}$, (fourth row) MERRA-2 temperature, and (fifth row) $T-T_{\mathrm{NAT}}$. Heavy dashed curves denote mean location of the vortex edge. Adapted from Pitts et al. (2018).

Figure 24. (a) Antarctic, May-November and (b) Arctic, November-March PSC column occurrence frequencies for CALIOP (2006-2017, black solid) and SAM II (1979-1989, red dashed), along with 1- $\sigma$ uncertainties in the multi-year means. Panel (c) shows the volume fraction of temperatures below $T_{\mathrm{NAT}}$ based on ERA-Interim reanalysis data for the CALIOP (black solid) and SAM II (red dashed) time periods, again with 1- $\sigma$ uncertainties in the means. (a) and (b) adapted from Pitts et al. (2018).

Figure 25. Multi-decadal time series of ground-based lidar and CALIOP season-long PSC sighting frequencies for (a) McMurdo, (b) Dumont d'Urville, and (c) Ny-Ålesund. Vertical bars are $2-\sigma$ uncertainties of the sample means.

Figure 26. Schematic description of different PSC formation pathways reflecting the current state of scientific understanding. Red numbers next to individual arrows are referred to in the remainder of this section and in Section 6, describing the formation pathways in detail. Circles: liquid droplets; hexagons: NAT or ice crystals; black triangles: solid nuclei (e.g. meteoritic). PSC particle compositions observed in the atmosphere include SSA, STS, NAT, and ice. Aerosol droplets that contain a foreign nucleus are symbolized with a triangle surrounded by a circle. The temperatures indicated for $T_{\mathrm{STS}}, T_{\mathrm{ice}}$ and $T_{\mathrm{NAT}}$ are approximate, reflecting typical polar stratospheric conditions. Note that some arrows are unidirectional (i.e. the opposite direction is kinetically blocked), while others are bidirectional. Figure adapted from Hoyle et al. (2013) and Engel et al. (2013).

Figure 27. Volumes and concentrations of STS droplets as function of temperature. (a) Black dots: Arctic measurements by Dye et al. (1992) on 24 January 1989 at 19 km altitude. Lines: thermodynamic model calculations of the volumes of various types of stratospheric particles in thermodynamic equilibrium with the gas phase; dotted line $=\mathrm{SSA}$; dashed line $=$ NAT particles; thick red line $=$ STS. Calculated volumes assume 5 ppmv $\mathrm{H}_{2} \mathrm{O}$ and 10 ppbv total $\mathrm{HNO}_{3}$ at 55 $\mathrm{hPa}$, i.e. about $19 \mathrm{~km}$ altitude. (b) Corresponding weight fractions in STS droplets. Solid curves: $\mathrm{H}_{2} \mathrm{SO}_{4}, \mathrm{HNO}_{3}, \mathrm{HCl}$ (remaining fraction to balance $100 \mathrm{wt} \%$ is $\mathrm{H}_{2} \mathrm{O}$ ). Dashed line corresponds to $\mathrm{H}_{2} \mathrm{SO}_{4}$ in binary $\mathrm{H}_{2} \mathrm{SO}_{4}-\mathrm{H}_{2} \mathrm{O}$ droplets. Figure adapted from Carslaw et al. (1994).

This article is protected by copyright. All rights reserved. 
Figure 28. (a) Differential number size distribution derived from FSSP-100 forward scattering measurements and total $\mathrm{NO}_{\mathrm{y}}$ in situ measurements during a PSC encounter during RECONCILE. (b) Number size distribution derived from Cloud Droplet Probe (CDP) measurements during ESSenCe. (c) Histogram of number concentration for diameters above $1.9 \mu \mathrm{m}, 4.7 \mu \mathrm{m}$ and 12.5 $\mu \mathrm{m}$ recorded within 11.6 flight hours during six flights inside PSCs. (a) and (b) adapted from Molleker et al. (2014); (c) is a synthesis of results from Molleker et al. (2014).

Figure 29. (a) MIPAS-STR observation showing a characteristic signature of large highly aspherical $\beta$-NAT particles during the ESSenCe flight on 11 December 2011 (black). Modeled spectra involving Mie calculations of spherical particles (red/blue) and T-Matrix calculations of highly elongated particles (green) consisting of $\beta$-NAT. (b) Red: Lognormal size distribution constrained by in situ observations and used for modelling the red spectrum in panel (a). Blue: Optimized size distribution after scaling to smaller sizes and used for modelling the blue and green spectra in panel (a). The combination of the opmized size distribution and assuming highly aspherical particles results in best agreement with the observation (cp. green and black spectra in panel (a)). Modified from Woiwode et al. (2016), their Figures 14a and 9b.

Figure 30. Median vertical profiles of submicron-sized particle abundance and non-volatile fraction $f$ at $250^{\circ} \mathrm{C}$, i.e. refractory particles, with 25 th and 75 th percentiles (bars) as function of potential temperature. Where whiskers are not visible, the percentile range is smaller than symbol size. Data are attributed to either originate from inside (dots) or outside the vortex (circles), derived from $\mathrm{N}_{2} \mathrm{O}$ as vortex tracer. Aerosol mixing ratios $N_{\text {total }}$ (first column) and the resulting fraction $f$ of particles featuring temperature stability at $250^{\circ} \mathrm{C}$ (second column). Upper row: ESSENCE 2011. Center row: EUPLEX 2003. Bottom row: RECONCILE 2010. Figure adapted from Weigel et al. (2014).

Figure 31. (a) CALIOP (blue) and scaled MIPAS (green) daily PSC volumes from 2002-2018 for Arctic PSC seasons, defined as December-March (same as Figure13b). Periods without data are marked in gray. (b) ERA-Interim estimated daily PSC volumes with $T<T_{\text {NAT }}$ (thin black line) and $T<T_{\mathrm{NAT}}-3.5 \mathrm{~K}$ (shaded blue). The red horizontal bars show the winter-long timeintegrated PSC volume (units $\mathrm{km}^{3} \times \mathrm{d}$ ) within the Arctic vortex, in which the temperature is below $T_{\text {NAT }}$ (adapted from WMO, 2018).

Figure 32. Composite maps of the geographic distribution of wave activity, PSCs and nitric acid compiled from satellite measurements during 28 May to 2 June 2008 for latitudes poleward of $58^{\circ}$. (a) Wave activity as represented by the largest AIRS brightness temperature variances. Also overlaid are isolines of the Montgomery stream function in gray, and the $189 \mathrm{~K}$ and $192 \mathrm{~K}$ temperature contours in blue-white and green-white, respectively. (b) MIPAS PSC composition classes at $22+/-2 \mathrm{~km}$. (c,d) CALIOP PSC composition classes at $22+/-2 \mathrm{~km}$. (e) $\mathrm{MLS} \mathrm{HNO}_{3}$ at $32 \mathrm{hPa}$. A dashed latitude circle at $82^{\circ} \mathrm{S}$ indicates the limit of the CALIOP and MLS observations. Diamond symbols in (b) and (d) indicate the MIPAS ice and CALIOP wave ice locations used for the initialization of the forward trajectories shown in Figure 33. Square symbols in (b) indicate MIPAS PSC detections that are unable to be specified (unspec). On 31 May MIPAS was operated in an instrument mode dedicated to the upper atmosphere and only provided limited coverage of the lower stratosphere.

Figure 33. Composite map of the geographic distribution of NAT and ice PSCs compiled from satellite measurements (Figure $32(\mathrm{~b}, \mathrm{c})$ ) during 28 May to 2 June 2008 for latitudes poleward of $58^{\circ}$. Forward trajectories on the $500 \mathrm{~K}$ potential temperature surface were launched from the

This article is protected by copyright. All rights reserved. 
MIPAS ice locations (Figure 32 (b)) and CALIOP wave ice locations (Figure 32 (c)) indicated here by the larger diamond symbols. The color scale indicates both the PSC observation times and the time along the trajectory tracks, such that trajectories passing near or intersecting with MIPAS or CALIOP observation tracks at near coincident times have similar colors. Trajectory calculations were based on wind fields from the Goddard Earth Observing System, Version 5 (GEOS-5).

Figure 34. (top) The observed backscatter ratio at $1064 \mathrm{~nm}$ measured by lidar onboard a research aircraft flying across Scandinavia on 25 January 2000 (color) along with potential temperature from an MM5 simulation (10K intervals). MM5 is a regional mesoscale model (a nonhydrostatic weather prediction model). The vertical line marks the position of Kiruna, Sweden, $68^{\circ} \mathrm{N}, 20^{\circ} \mathrm{E}$. (bottom) Elevation of the topography below the flight leg (gray shading: digital topography in 1 $\mathrm{km}$ horizontal resolution; black line, topography in the innermost nested domain of MM5). Figure from Dörnbrack et al. (2002).

Figure 35. Measured (left) and mesoscale modelled (right) estimates of the $15 \mu \mathrm{m}$ Brightness Temperature (BT) perturbations (units K) over the Antarctic Peninsula corresponding to the $666.5 \mathrm{~cm}^{-1}$ AIRS channel. Figure adapted from Orr et al. (2015).

Figure 36. Chemical conditions in the ozone layer over Antarctica during the formation of the ozone hole derived from satellite measurements. Maps show polar orthographic projections nead $18 \mathrm{~km}$ altitude for late winter (September). For details see text. From Figure 7-3 of the WMO/UNEP 20 Questions and Answers (WMO, 2018).

Figure 37. Idealized sketch of the seasonal evolution of inorganic chlorine and temperature in the lower stratosphere inside the Antarctic vortex. Most of the inorganic chlorine is resident as $\mathrm{HCl}$ and $\mathrm{ClONO}_{2}$ as the vortex starts to form (upper panel). Once temperature falls below a critical threshold (lower panel), these reservoir species are converted to active chlorine species $\mathrm{Cl}, \mathrm{ClO}$, $\mathrm{ClOOCl}, \mathrm{Cl}_{2}$, and $\mathrm{HOCl}$. Denitrification and dehydration, the removal of $\mathrm{HNO}_{3}$ and $\mathrm{H}_{2} \mathrm{O}$ by the gravitational settling of large NAT and ice particles, also takes place. Rapid loss of $\mathrm{O}_{3}$ occurs after the return of sunlight, driven by the $\mathrm{ClO}+\mathrm{ClO}$ and the $\mathrm{BrO}+\mathrm{ClO}$ catalytic cycles. After PSC formation ceases in early spring in the Antarctic vortex, active chlorine is converted back to the reservoir gases via $\mathrm{Cl}+\mathrm{CH}_{4} \rightarrow \mathrm{HCl}+\mathrm{CH}_{3}$ and $\mathrm{ClO}+\mathrm{NO}_{2}+\mathrm{M} \rightarrow \mathrm{ClONO}_{2}+\mathrm{M}$. After the vortex circulation is disrupted, $\mathrm{O}_{3}$ is replenished by mixing with extra vortex air and the partitioning of inorganic chlorine back to $\mathrm{HCl}$ and $\mathrm{ClONO}_{2}$ is re-established. For a reality check of this idealized sketch, see Figure 41.

Figure 38. Recommended reactive uptake coefficients (reaction probabilities) $\gamma_{0}$ for key stratospheric heterogeneous processes as a function of temperature $\mathrm{T}$ on stratospheric sulfuric acid aerosols (SSA). The right axis shows approximate chemical lifetimes of the lesser abundant reactants $\mathrm{ClONO}_{2}, \mathrm{HOCl}, \mathrm{N}_{2} \mathrm{O}_{5}$ and $\mathrm{BrONO}_{2}$, assuming $10 \mathrm{~cm}^{-3}$ SSA droplets with $0.1 \mu$ m radius. Adapted from Figure 5-1 of Burkholder et al. (2015).

Figure 39. First-order loss rates for (a) $\mathrm{R} 5.1: \mathrm{ClONO}_{2}+\mathrm{HCl}$ and (b) $\mathrm{R} 5.2: \mathrm{ClONO}_{2}+\mathrm{H}_{2} \mathrm{O}$ for different parameterizations and aerosol types for typical stratospheric conditions ( $50 \mathrm{hPa}, 5 \mathrm{ppmv}$ $\mathrm{H}_{2} \mathrm{O}, 1$ ppbv $\mathrm{HCl}, 0.5$ ppbv $\mathrm{ClONO}_{2}, 10$ ppbv $\mathrm{HNO}_{3}, 0.15$ ppbv $\mathrm{H}_{2} \mathrm{SO}_{4}$ and 10 background SSA particles $\mathrm{cm}^{-3}$ ) in early winter, i.e. before denitrification and dehydration occurs. Solid blue lines depict STS, dashed blue lines SSA. Solid green and orange lines represent NAT particles with density $10^{-1} \mathrm{~cm}^{-3}$, representative of the rare dense NAT clouds ("enhanced NAT mixtures), dashed lines $10^{-4} \mathrm{~cm}^{-3}$, representative of the frequent tenuous NAT clouds ("NAT mixtures").

This article is protected by copyright. All rights reserved. 
Measurements by different laboratories are shown by the different colors. Cyan lines correspond to wave ice (solid, $10 \mathrm{~cm}^{-3}$ ) and ice particles (dashed, $0.01 \mathrm{~cm}^{-3}$ ). Figure adapted from Wegner et al. (2012).

Figure 40. Atmospheric measurements of $(\mathrm{ClO}+2 \times \mathrm{ClOOCl}) / \mathrm{Cl}_{\mathrm{y}}$ as a function of temperature. Data were acquired in situ in the lower stratosphere of the northern hemisphere during January to March 2000. Measurements acquired inside the Arctic polar vortex are in blue and outside the polar vortex are in green. Figure adapted from Wilmouth et al. (2018) based on the measurements of Wilmouth et al. (2006).

Figure 41. Seasonal evolution of chlorine reservoir species in the lower stratosphere inside the Antarctic vortex. Panels show vortex-core averages $\left(>75^{\circ} \mathrm{S}\right)$ on the $500 \mathrm{~K}$ isentrope for (a) $\mathrm{HCl}$ and (b) $\mathrm{ClONO}_{2}$ for different model simulations and satellite measurements. Green lines: observations by MLS and MIPAS. Red lines: CLaMS Lagrangian trajectory model. Blue lines: SD-WACCM global Eulerian model simulations with horizontal resolution of $1.2^{\circ}$. Magenta lines: TOMCAT/SLIMCAT chemical transport model with horizontal resolution of $1.2^{\circ}$. Adapted from Grooß et al. (2018).

Figure 42. PSC classification derived from CALIOP for a single satellite orbit between latitudes 60-82 ${ }^{\circ} \mathrm{N}$ on 18 January 2010 (left) and corresponding simulation by CLaMS (right). The solid and dashed lines in the panels enclose the regions with temperatures below $T_{\text {ice }}$ and $T_{\mathrm{NAT}}$, respectively. Adapted from Tritscher et al. (2019).

Figure 43. Comparison of (top row) CALIOP PSC measurements with (bottom row) SOCOL model results from the simulation using optimized parameters. To compare observations and simulations, the CALIOP PSC detection thresholds (Pitts et al., 2018) were applied to the calculated optical properties of the modeled PSCs. (a, c): 2-D histograms of CALIOP and SOCOL PSC data shown as fraction of all Antarctic PSCs in July 2007, plotted in the optical parameter space of Pitts et al. (2018). (b, d): Time series of CALIOP and SOCOL total PSCcovered area over the region $50^{\circ} \mathrm{S}-90^{\circ} \mathrm{S}$ as a function of altitude for the 2007 Antarctic PSC season.

Figure 44. Comparison of PSC areal coverage for the 2010 Antarctic winter derived from CALIOP data and simulated by WACCM. Left column (a1-a4): CALIOP observations. Center column (b1-b4): PSC coverage for different particle compositions (STS, NAT, ice) simulated directly by WACCM. Right column (c1-c4): PSC coverage modeled by WACCM for various CALIOP composition classes (STS, MIX, ice) identified using the CALIOP classification scheme of Pitts et al. (2018). Adapted from Zhu et al. (2017a).

This article is protected by copyright. All rights reserved. 

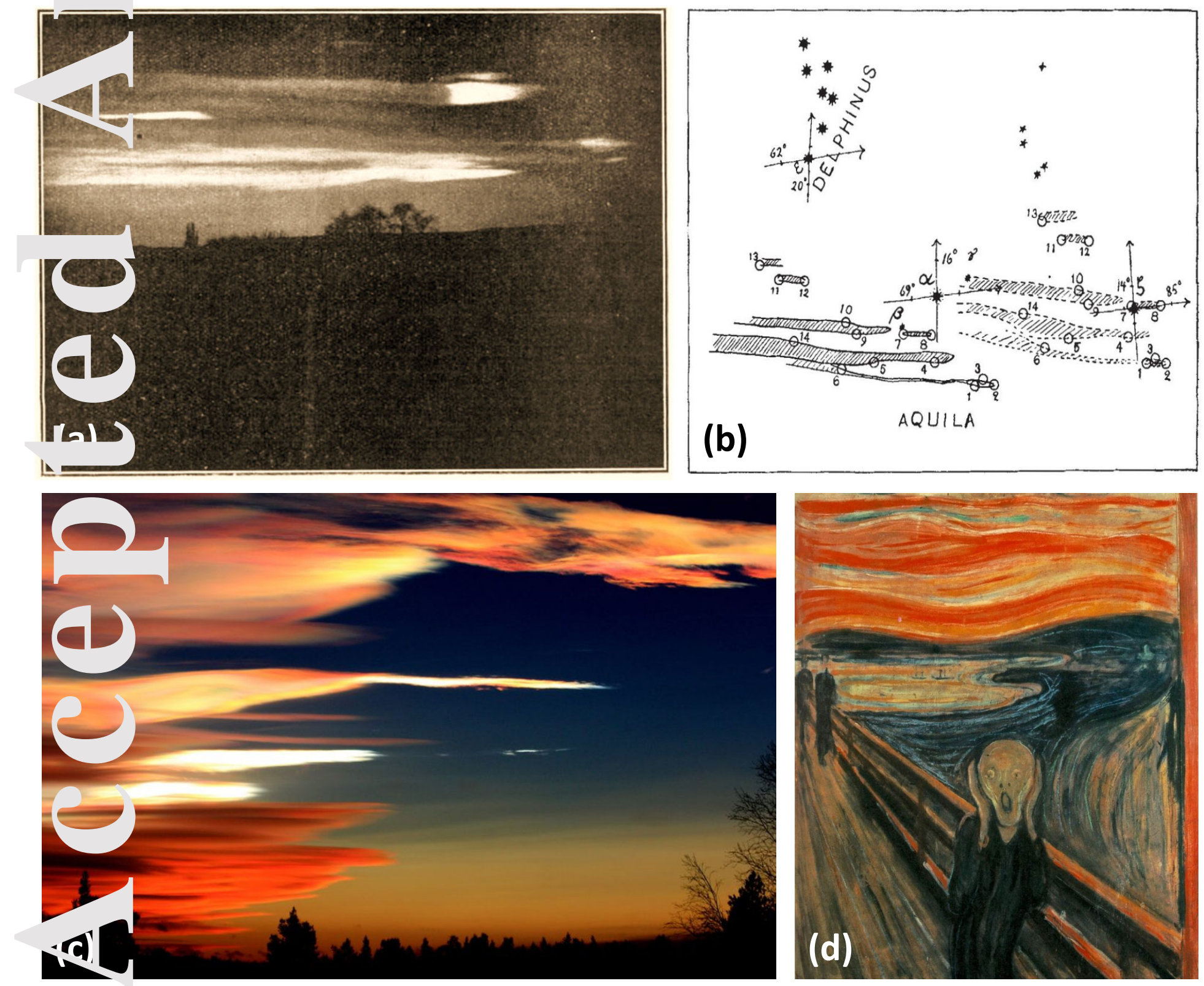

This article has been accepted for publication and undergone full peer review but has not been through the copyediting, typesetting, pagination and proofreading process, which may lead to differences between this version and the Version of Record. Please cite this article as doi: 10.1029/2020RG000702.

This article is protected by copyright. All rights reserved. 

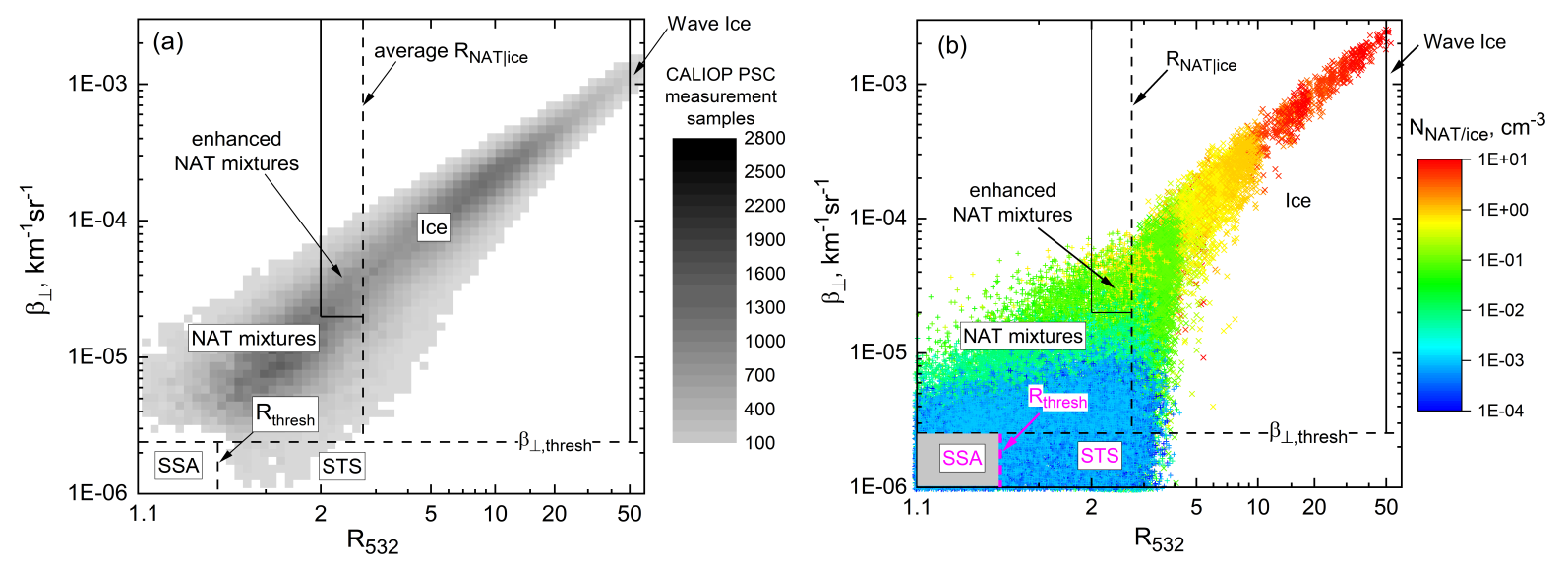

This article is protected by copyright. All rights reserved. 

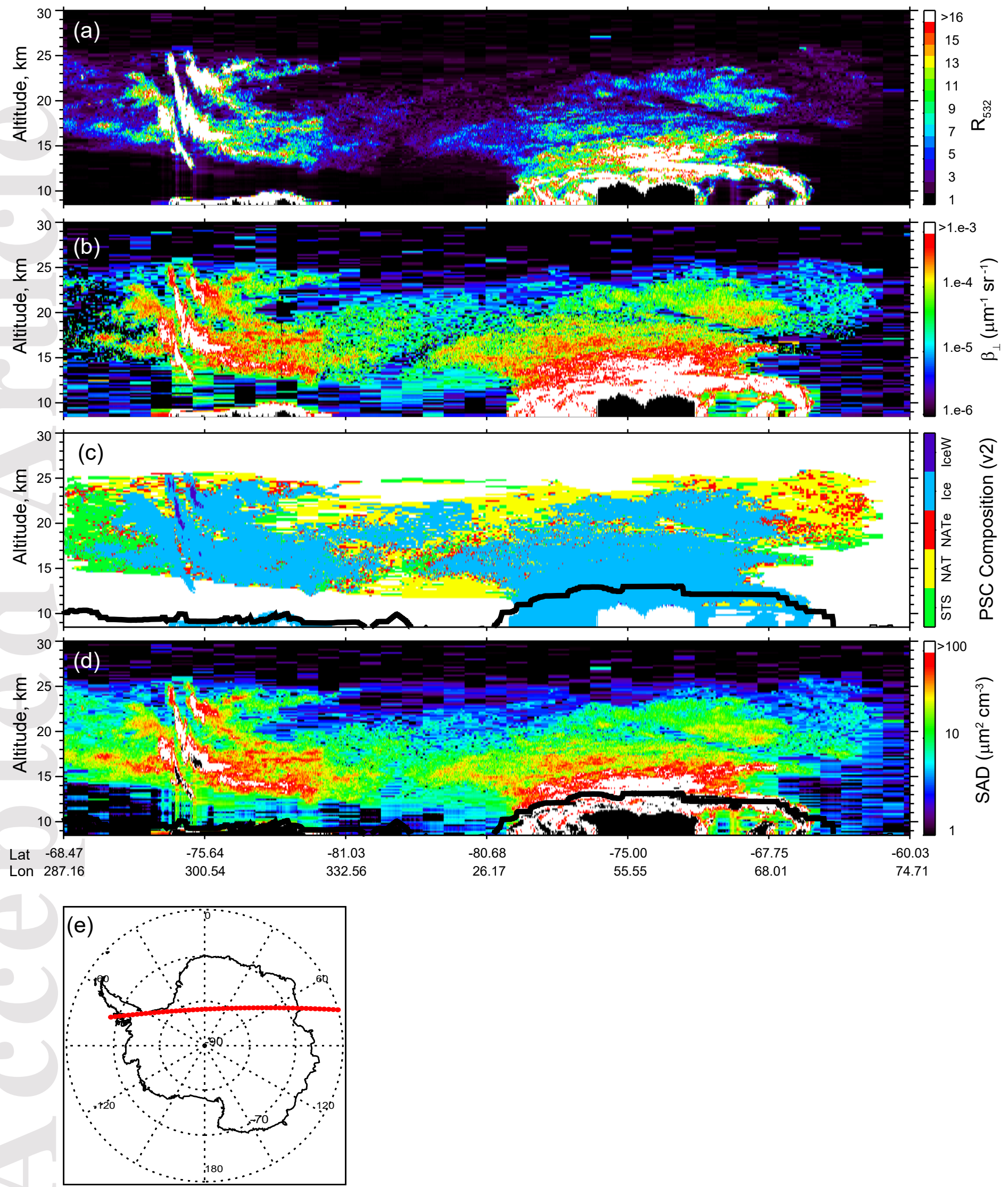

This article is protected by copyright. All rights reserved. 
(a)

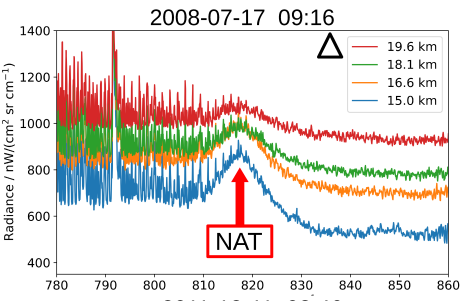

(b)

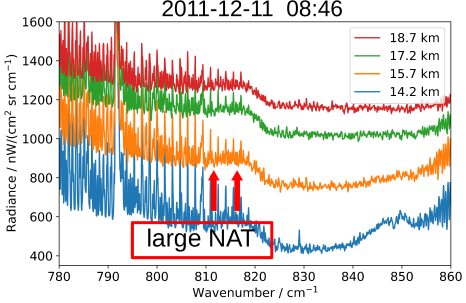

(c)

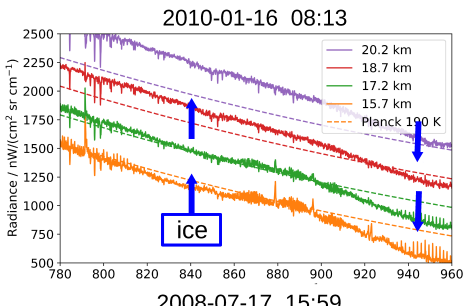

(d)

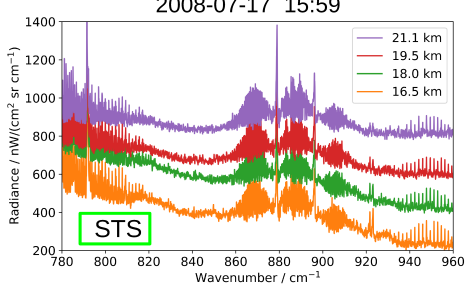

(e)

MIPAS-Classes 2008-07-17 [19.6-21.8] km

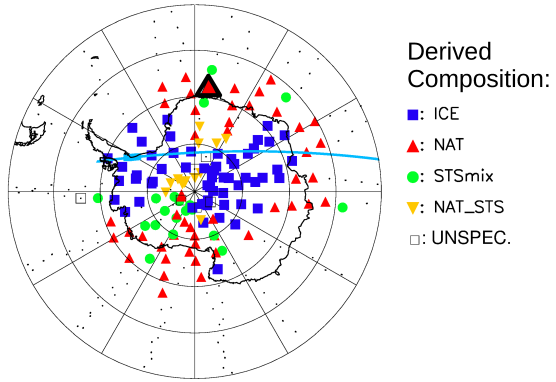

(f)

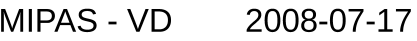

$21 \mathrm{~km}$

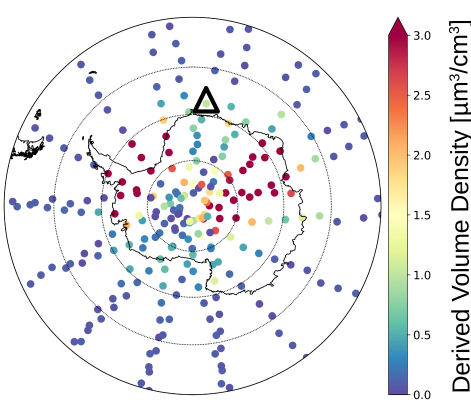

(g)

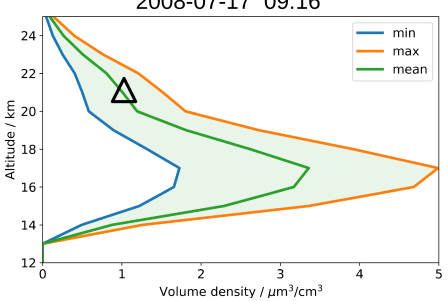

This article is protected by copyright. All rights reserved. 


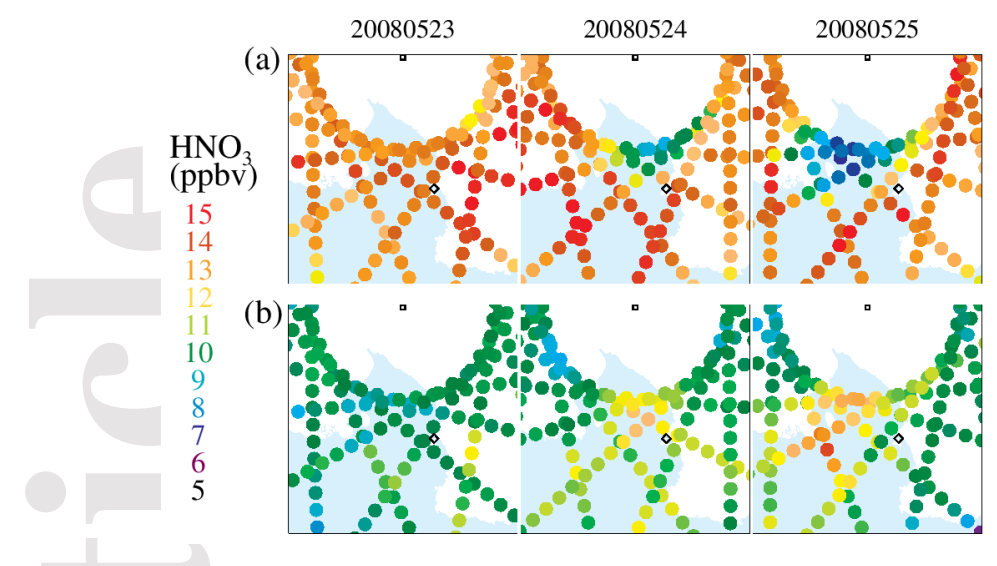

This article is protected by copyright. All rights reserved. 
(a) CALIOP PSC Composition

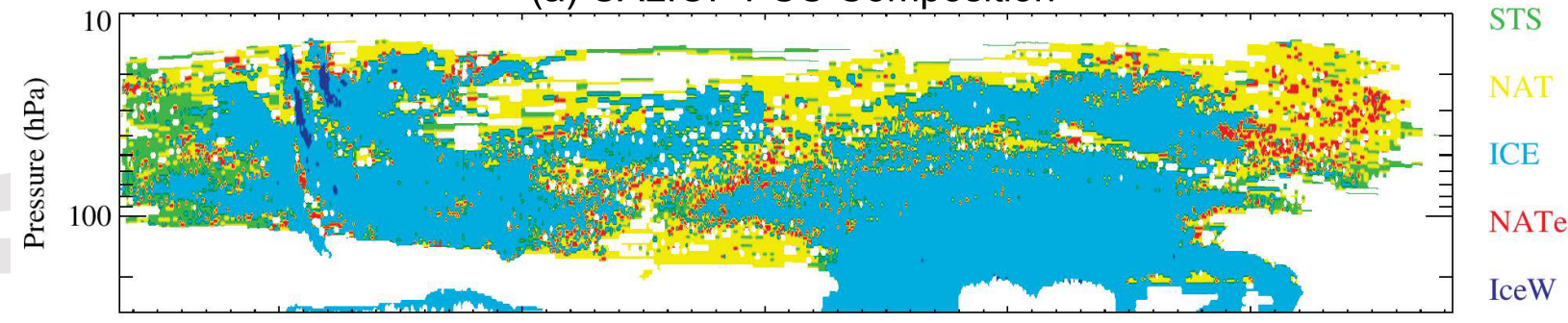

(b) $T_{\text {MERRA-2 }}-T_{\text {ICE }}$

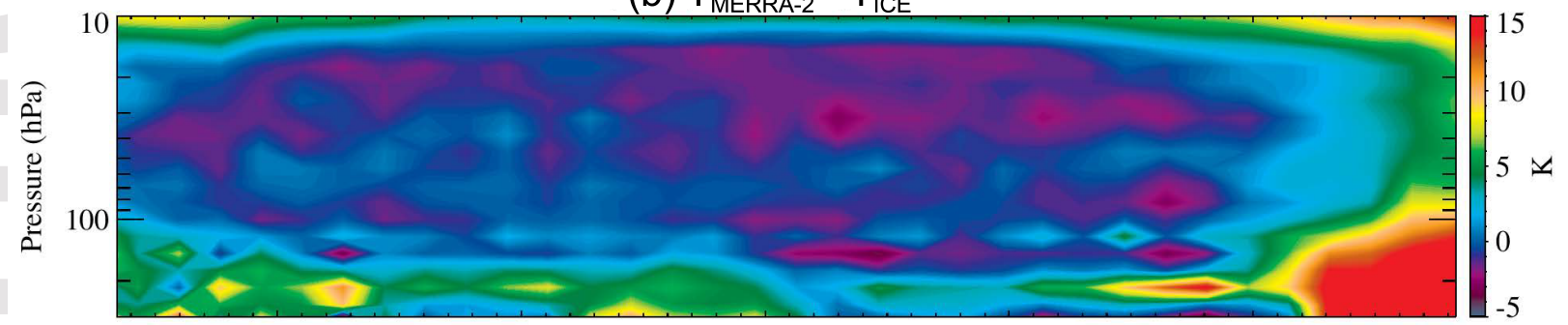

(c) $\mathrm{MLS} \mathrm{H}_{2} \mathrm{O}$

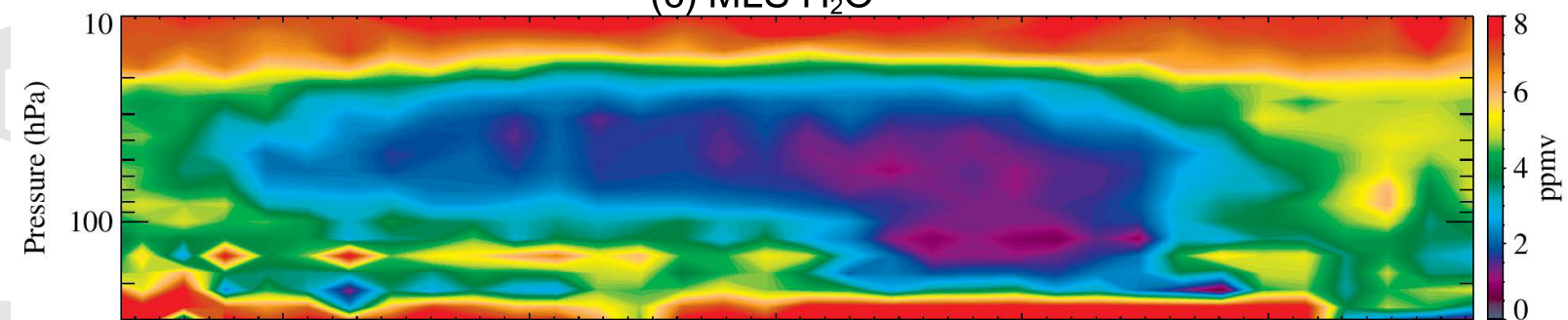

(d) $\mathrm{MLS} \mathrm{HNO}_{3}$

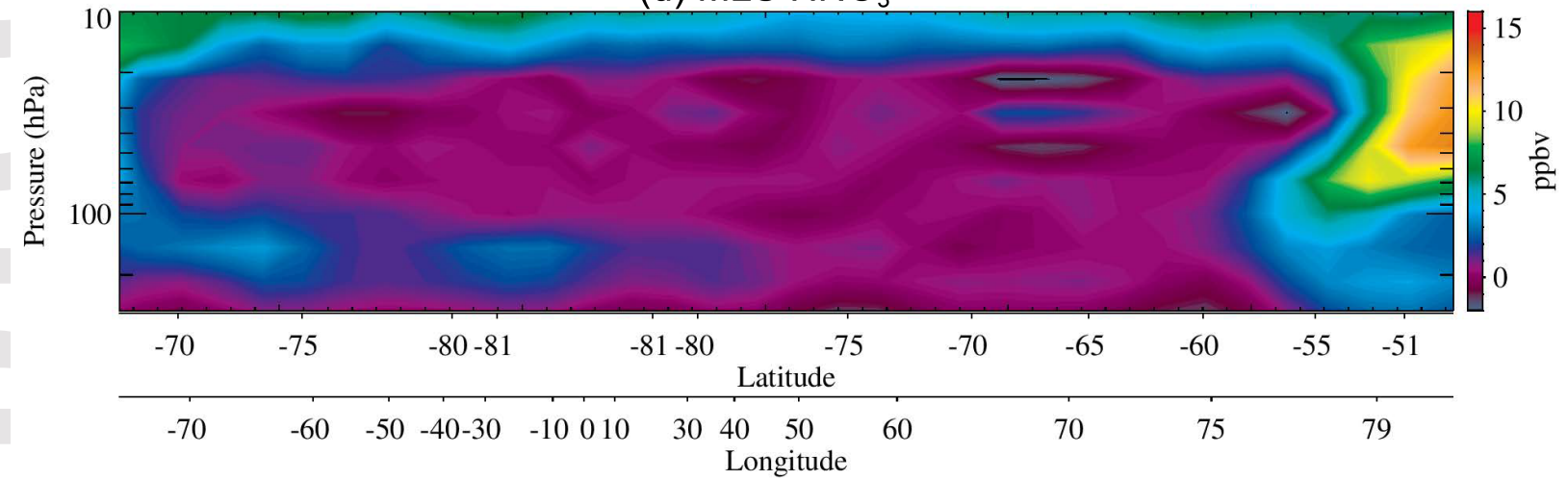

This article is protected by copyright. All rights reserved. 


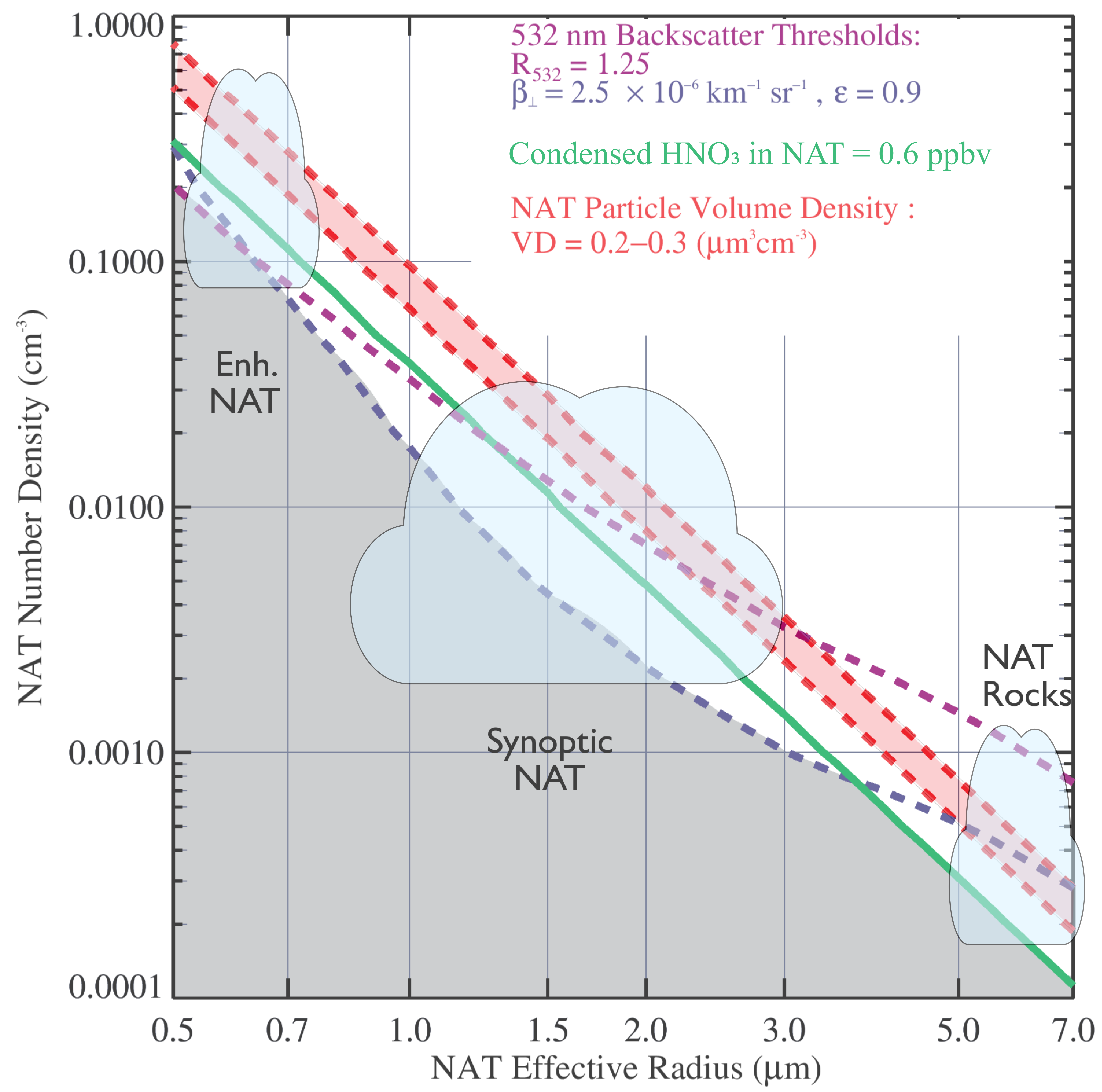




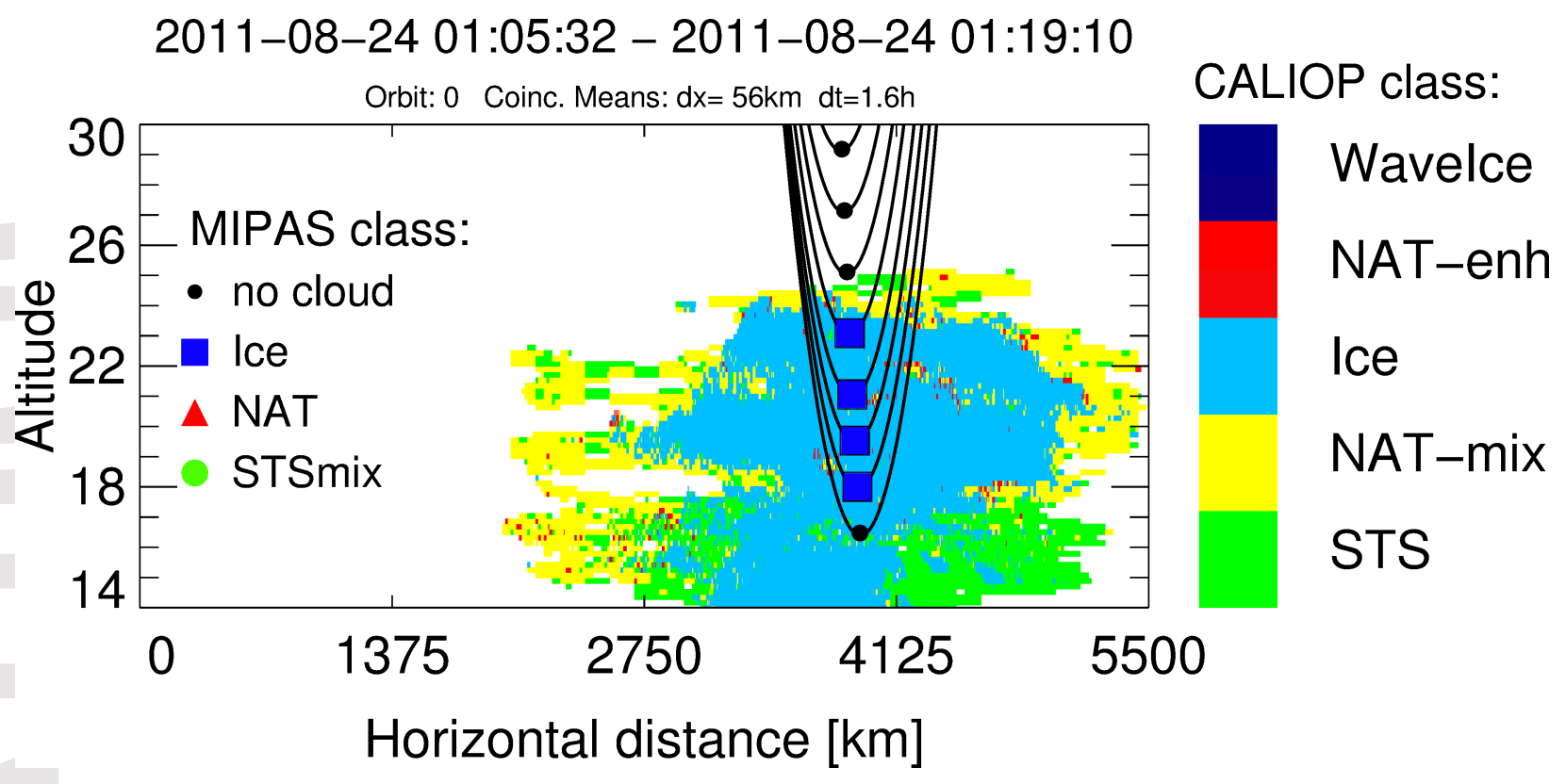

This article is protected by copyright. All rights reserved. 


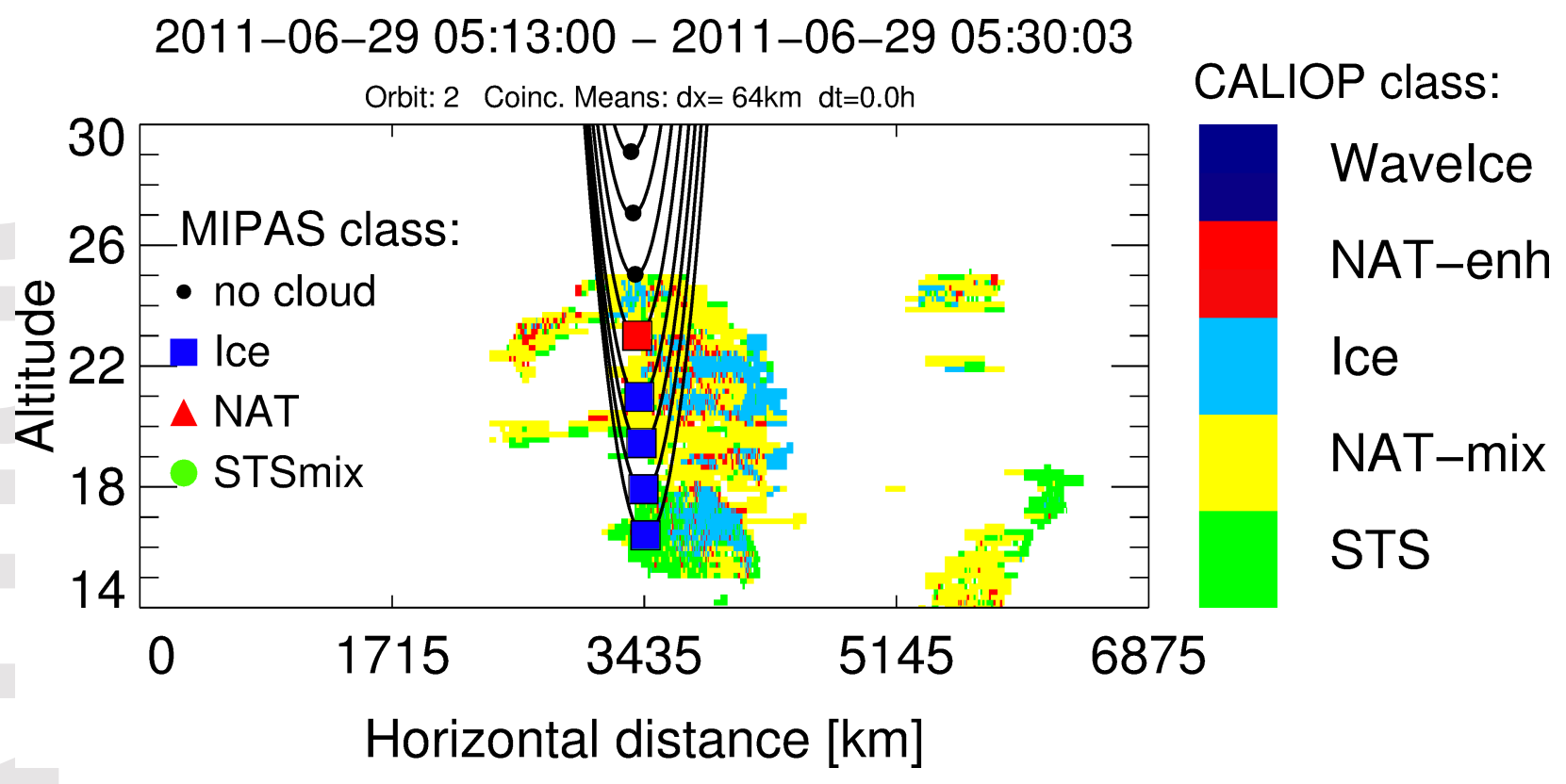

This article is protected by copyright. All rights reserved. 

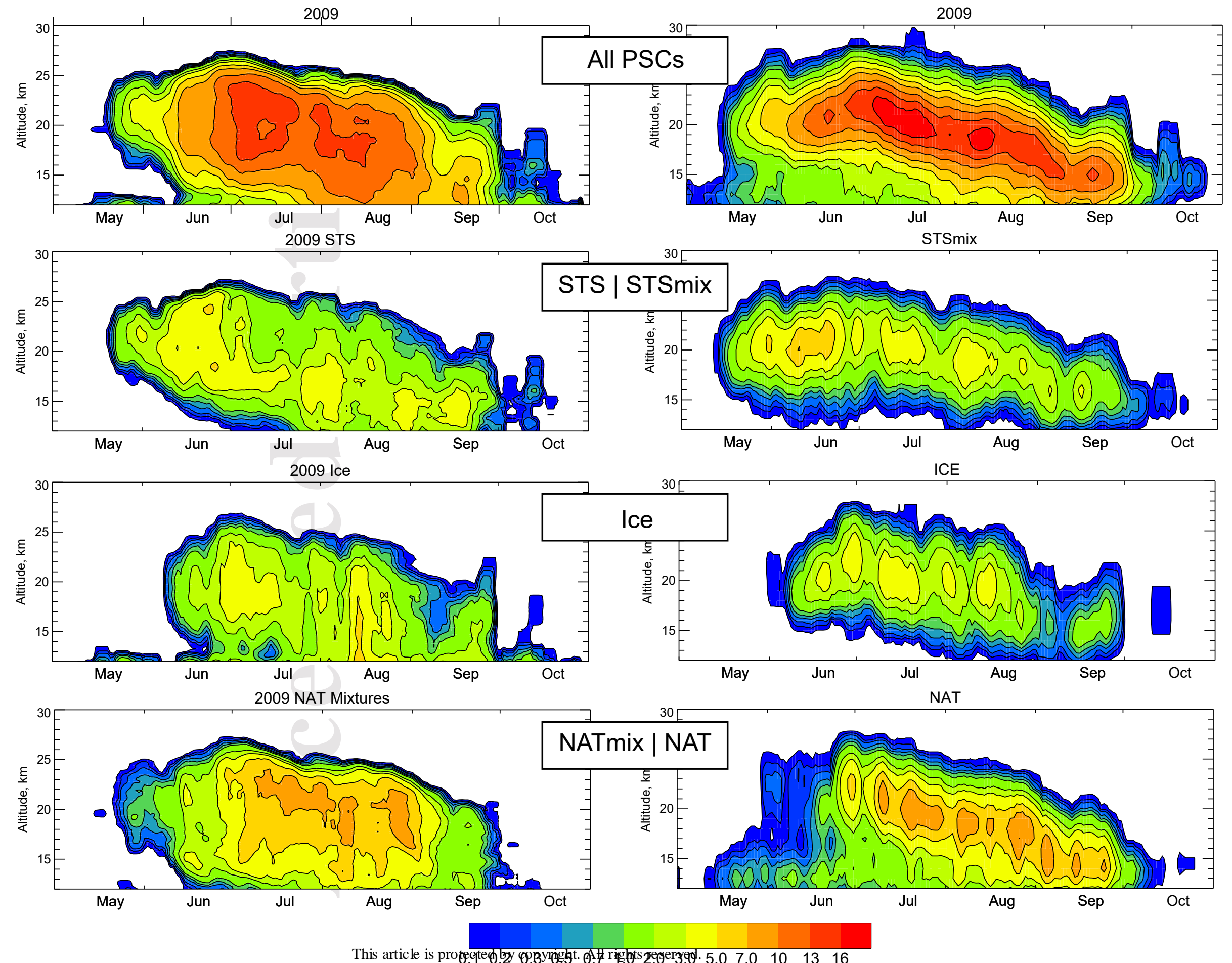

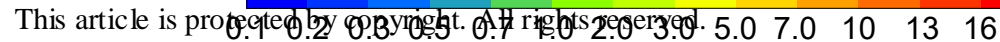



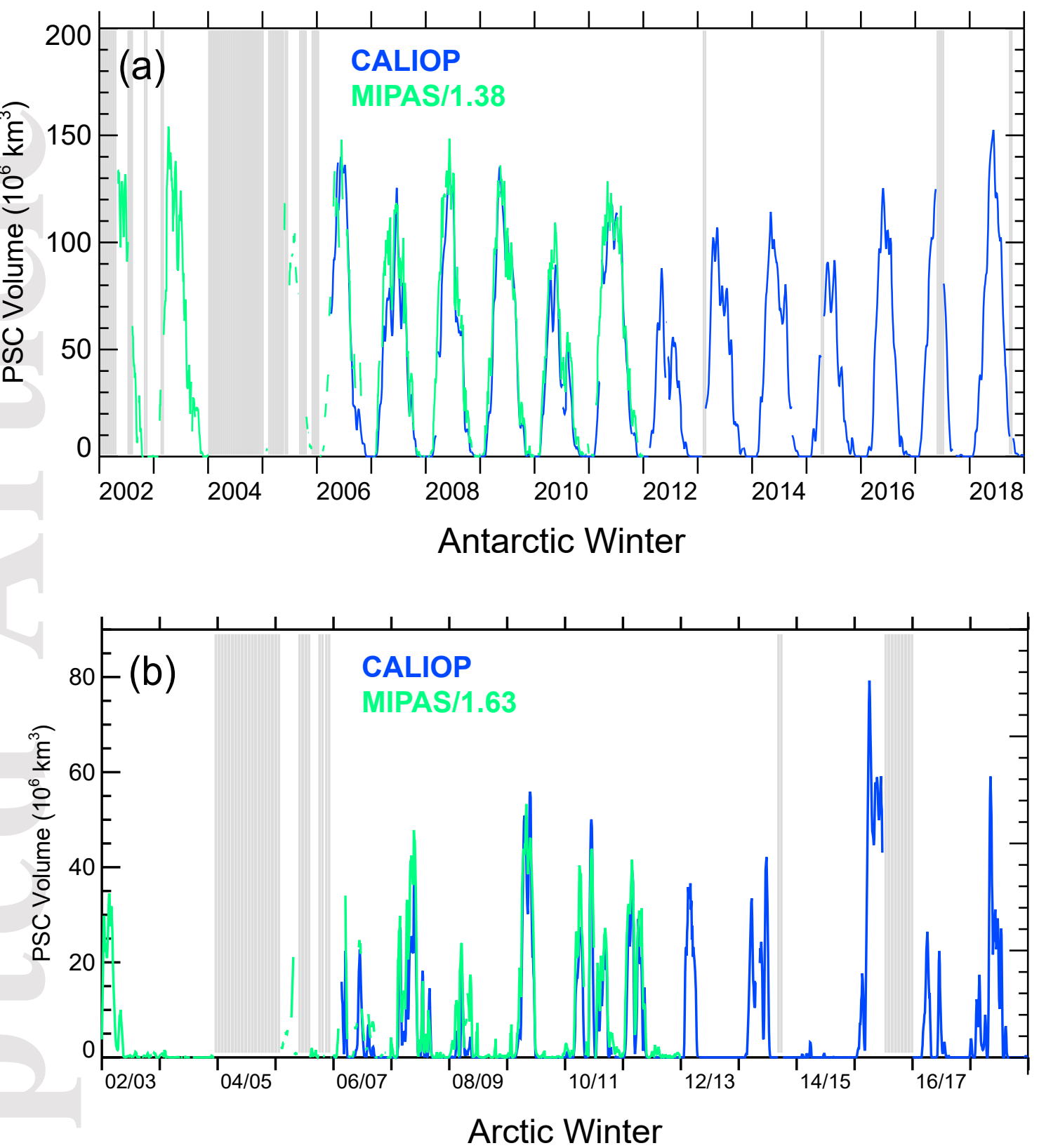

This article is protected by copyright. All rights reserved. 
(a)

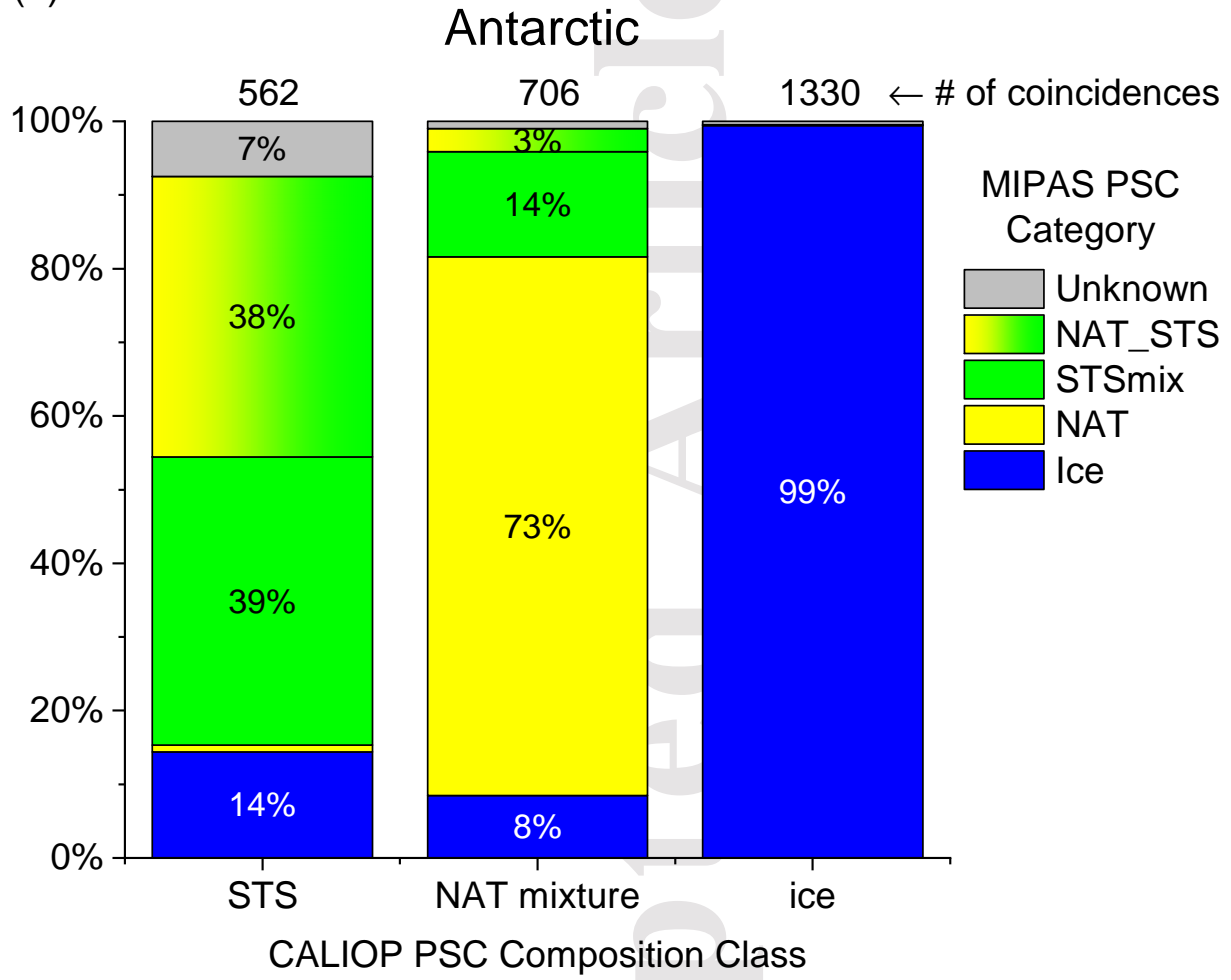

(b)

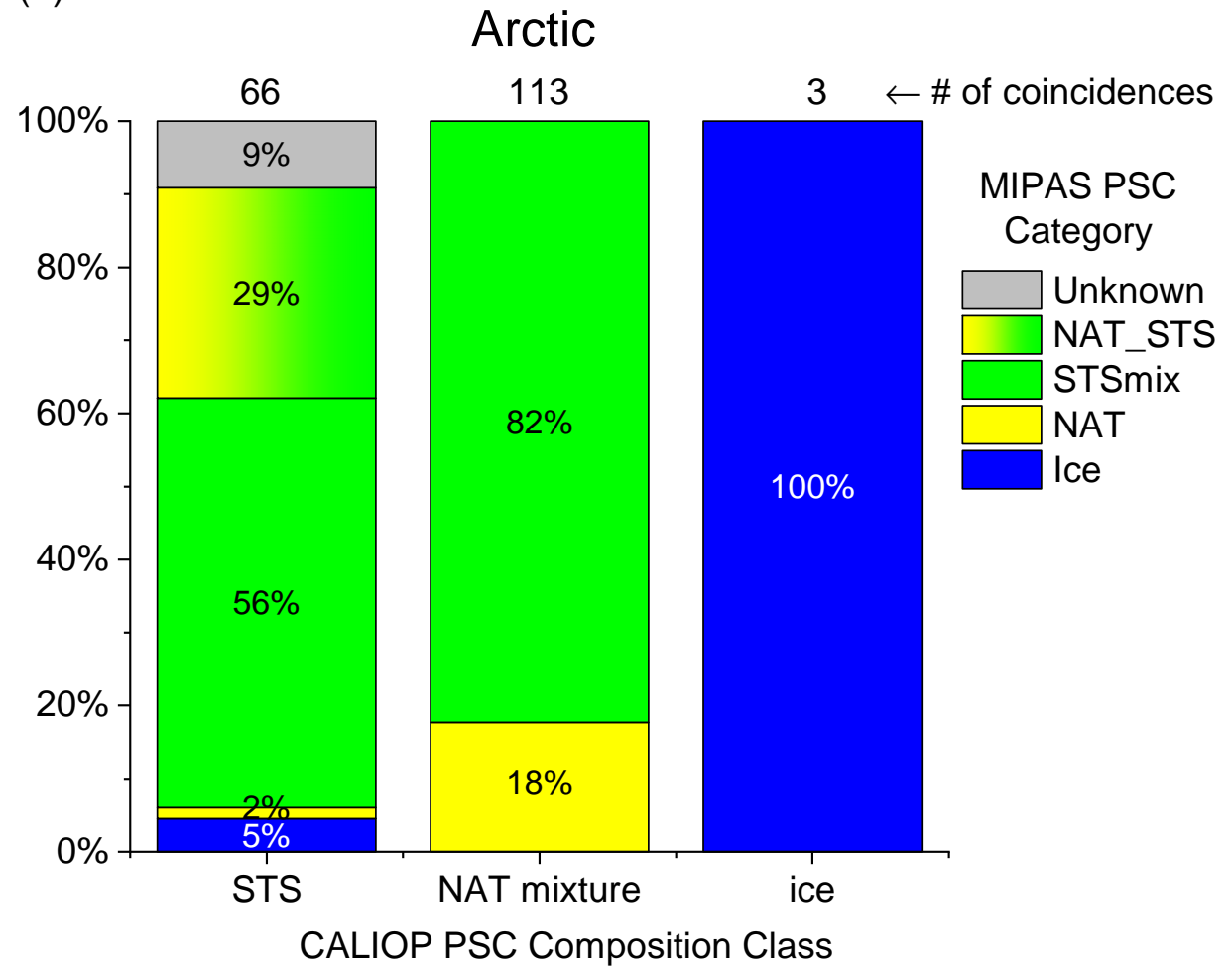


Antarctic 2006-2018
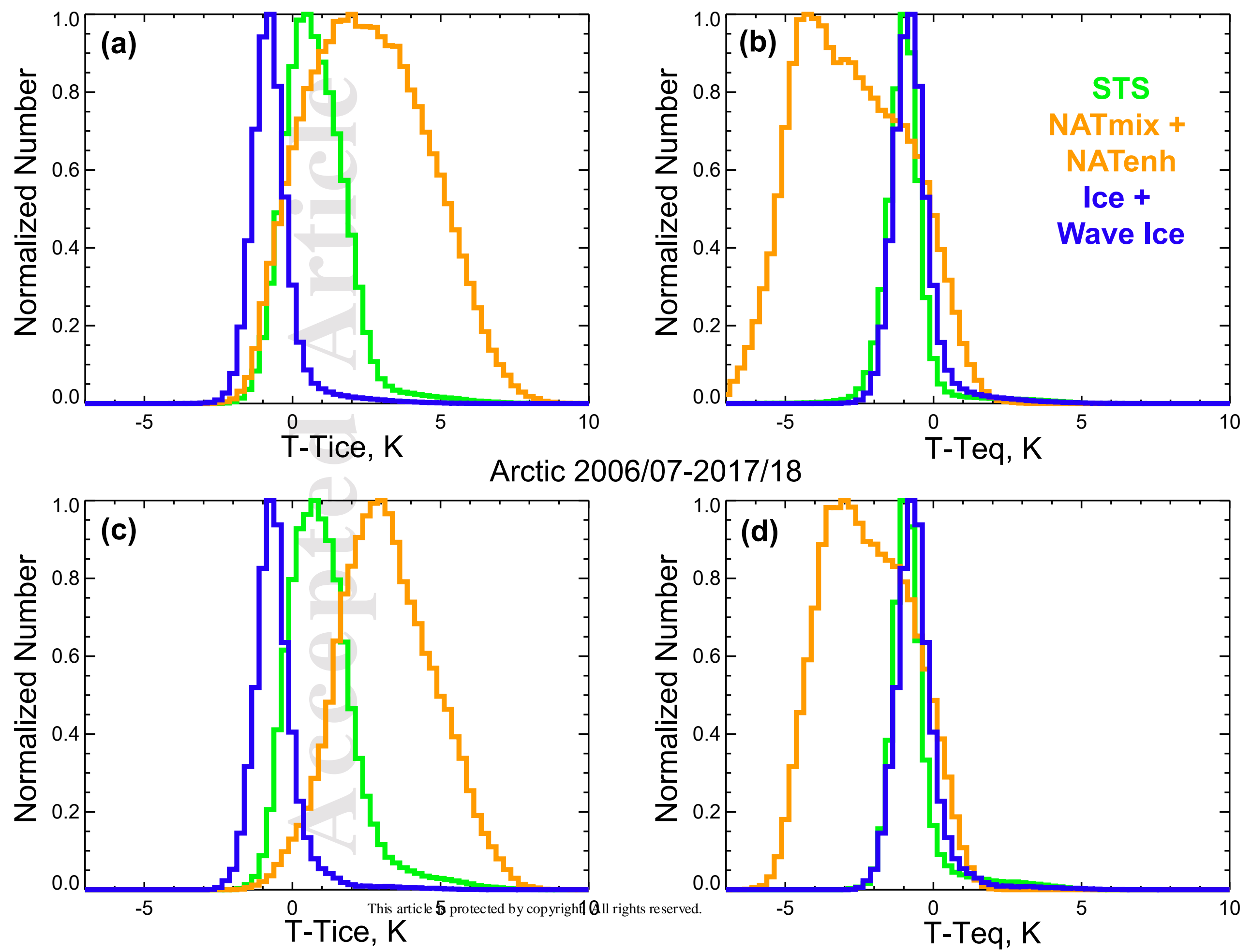

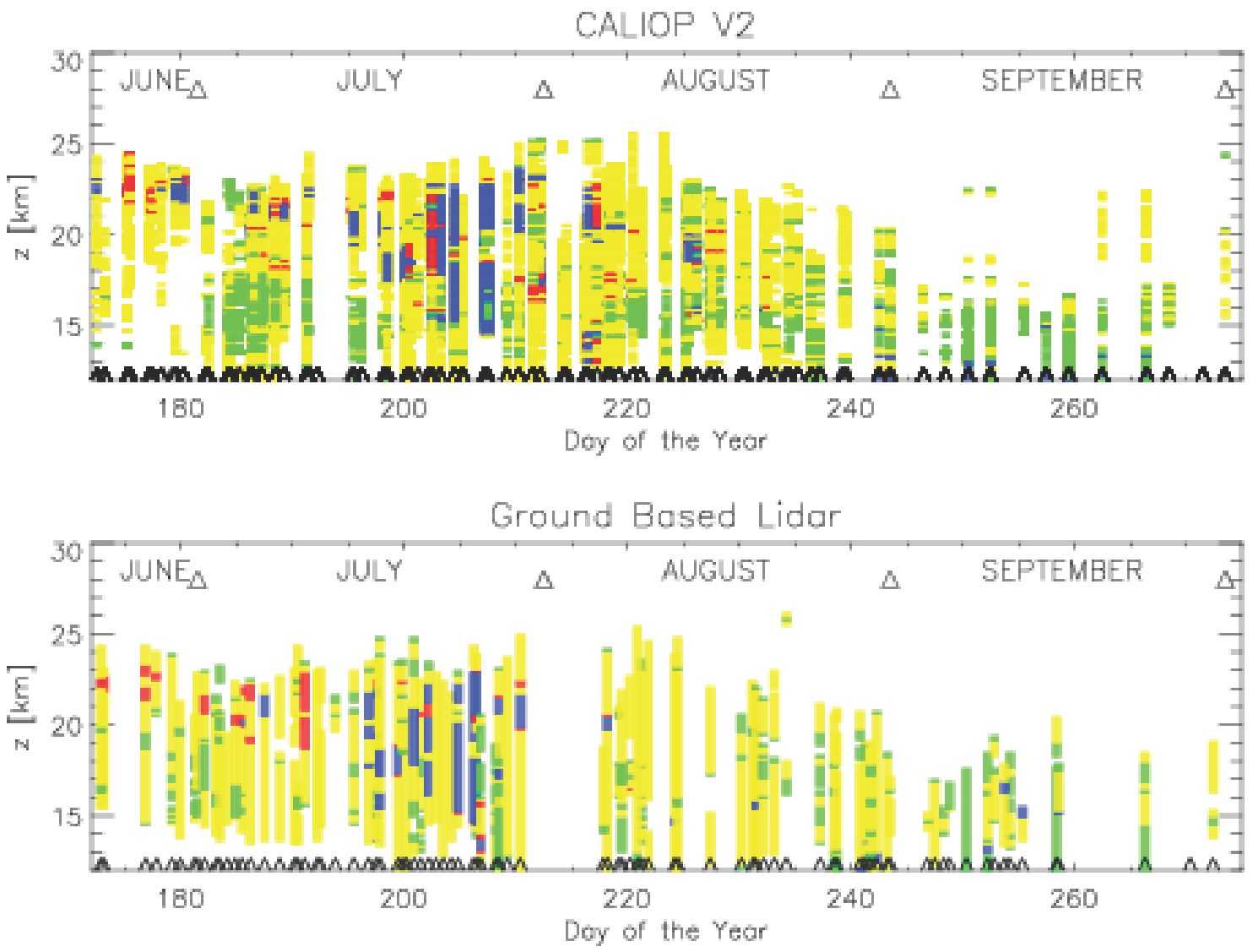

This article is protected by copyright. All rights reserved. 

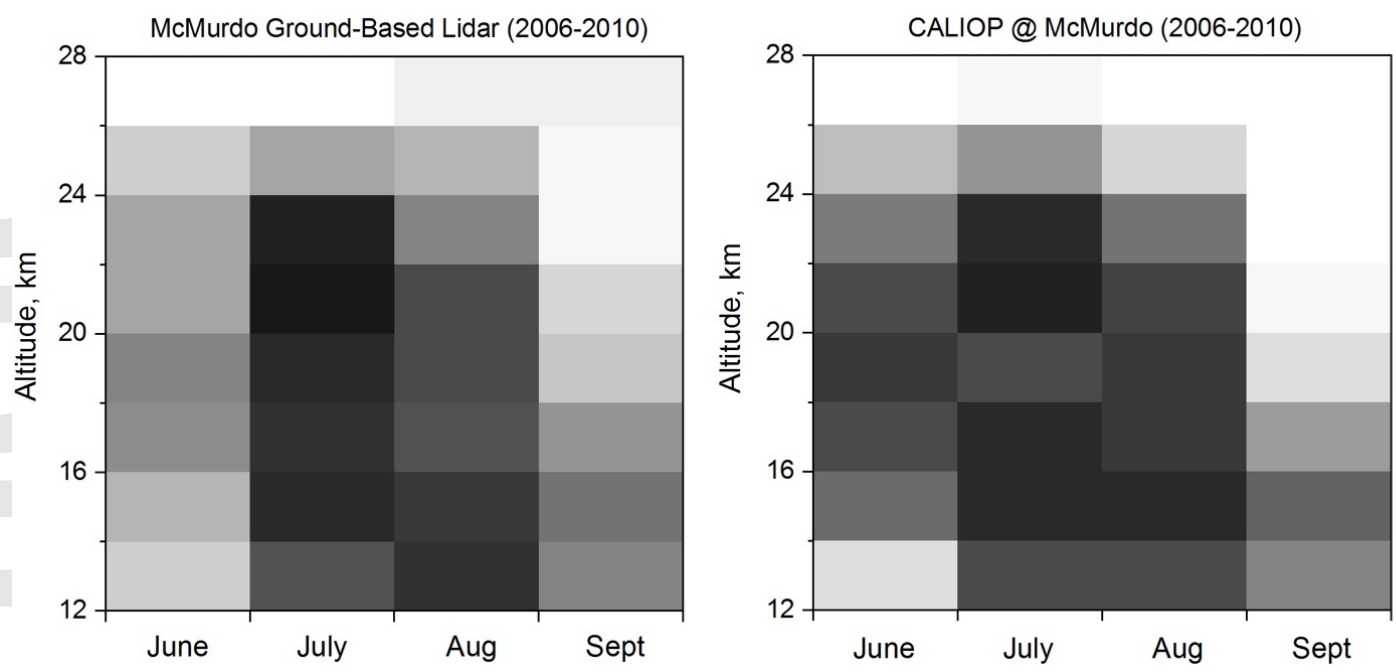

PSC
sighting frequency

10

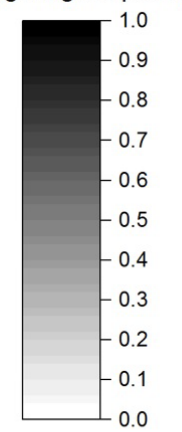

This article is protected by copyright. All rights reserved. 
(a) Antarctic

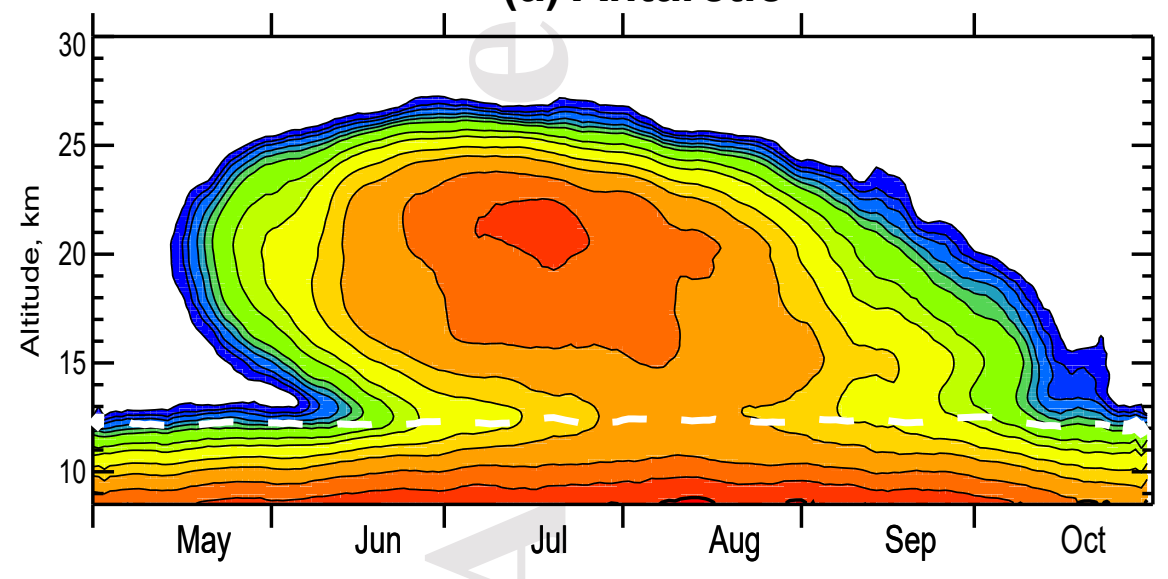

(b) Arctic

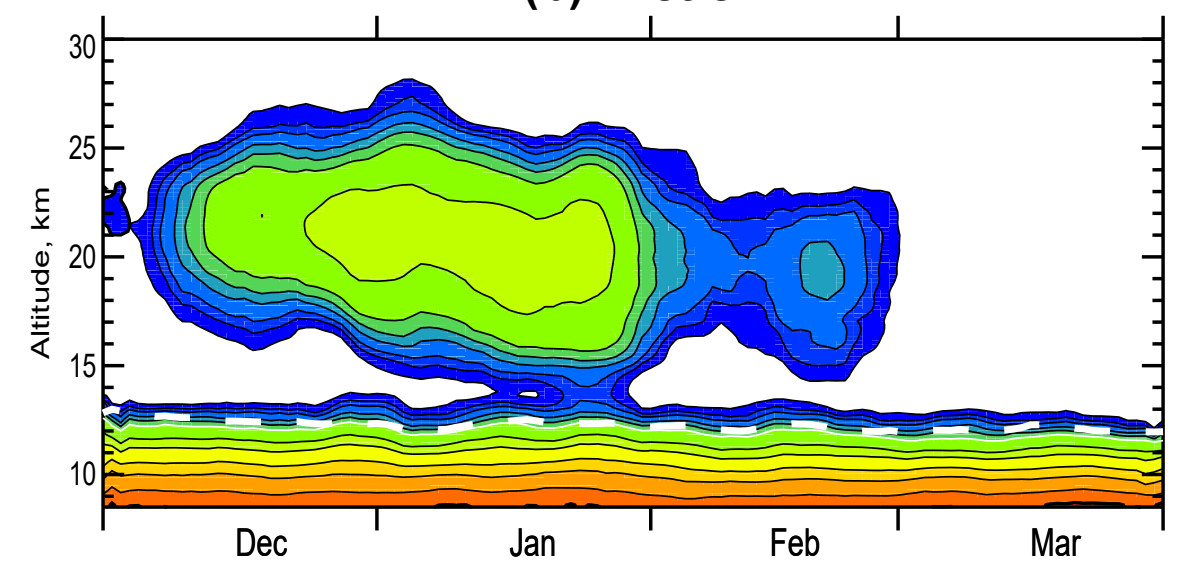

$\begin{array}{lllllllllllll}0.1 & 0.2 & 0.3 & 0.5 & 0.7 & 1.0 & 2.0 & 3.0 & 5.0 & 7.0 & 10 & 13 & 16\end{array}$

PSC Areal Coverage $\left(\times 10^{6} \mathrm{~km}^{2}\right)$ 

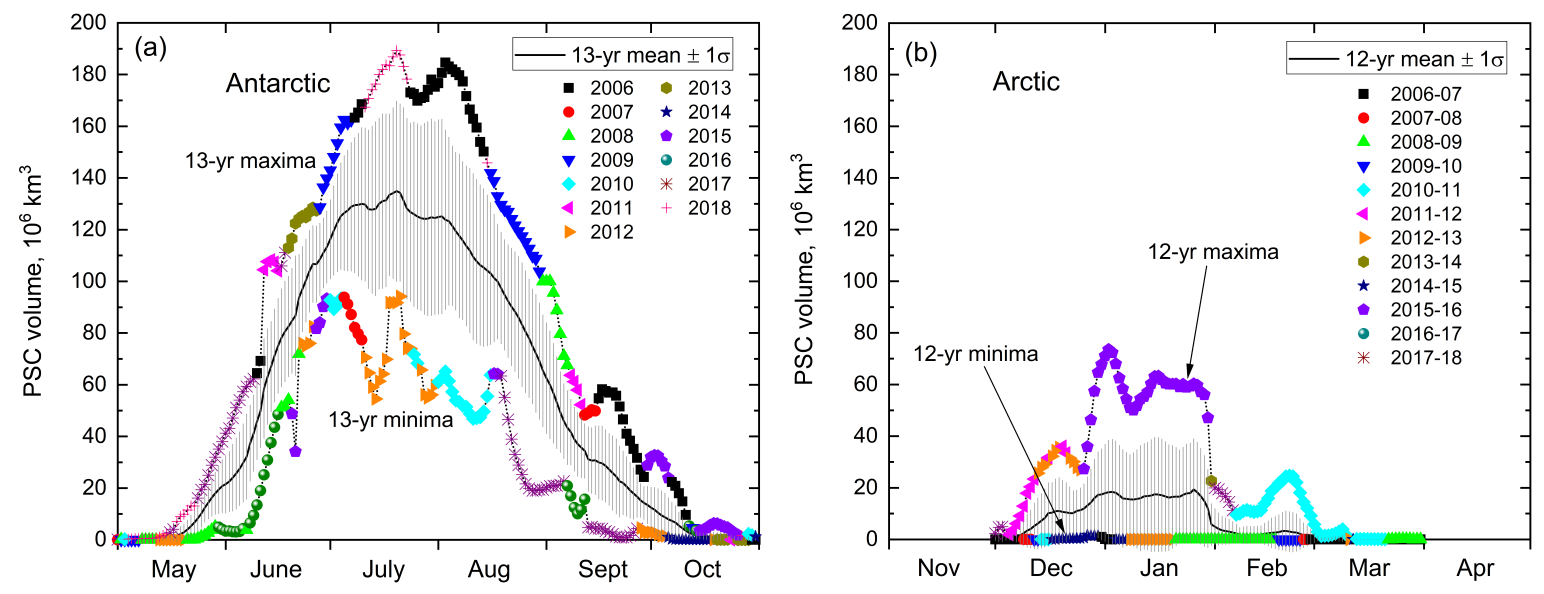

This article is protected by copyright. All rights reserved. 

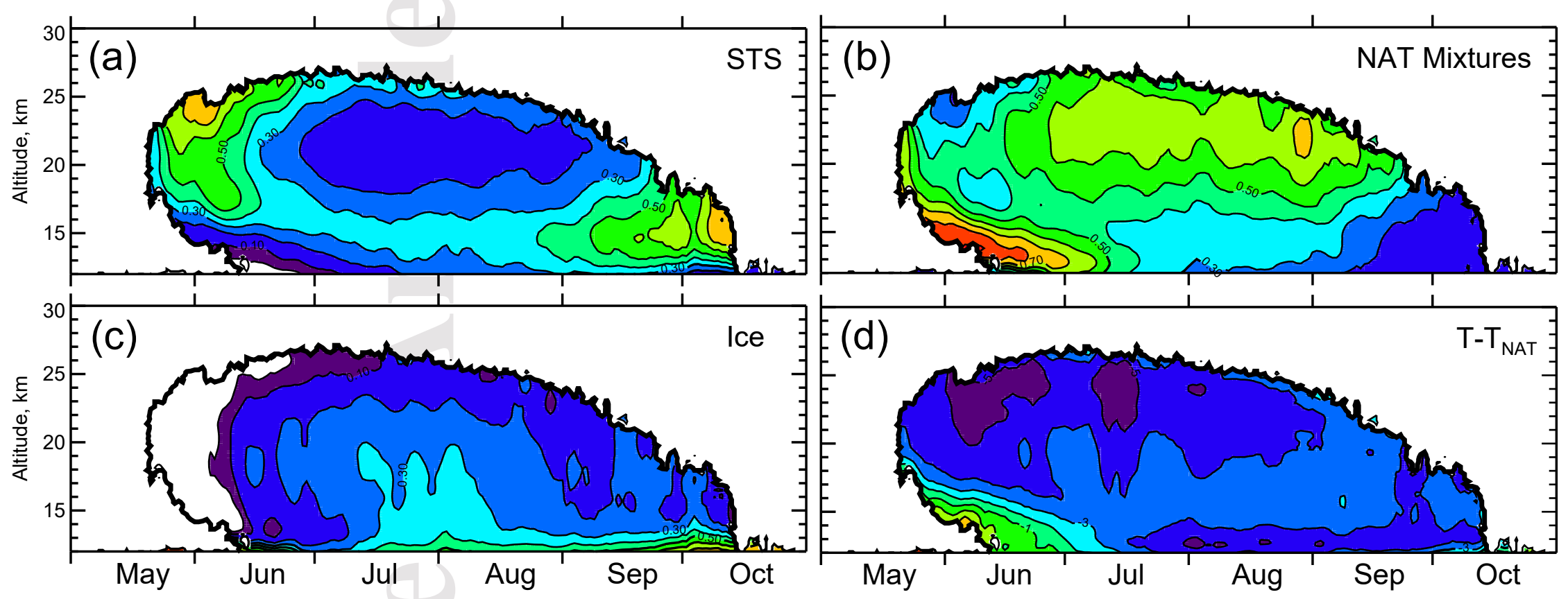

Fraction of Total PSC Area

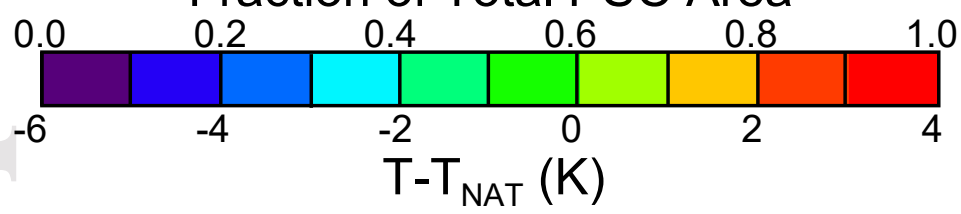



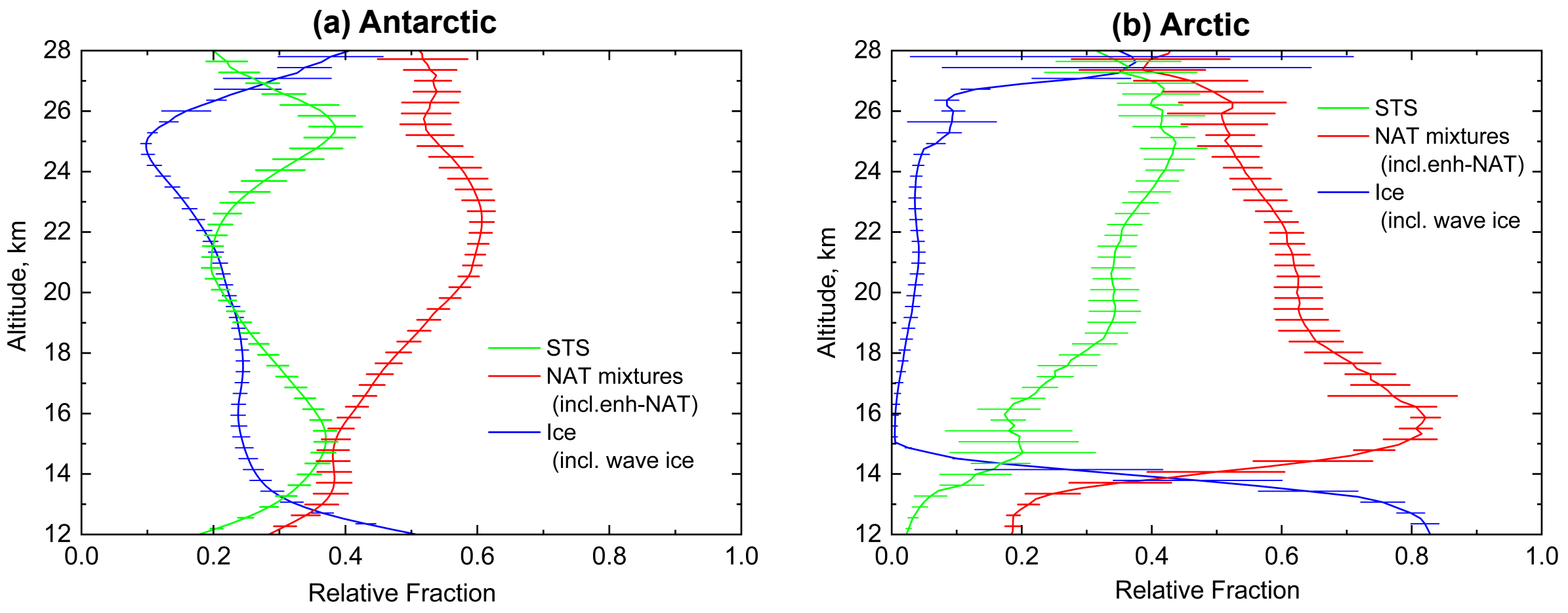

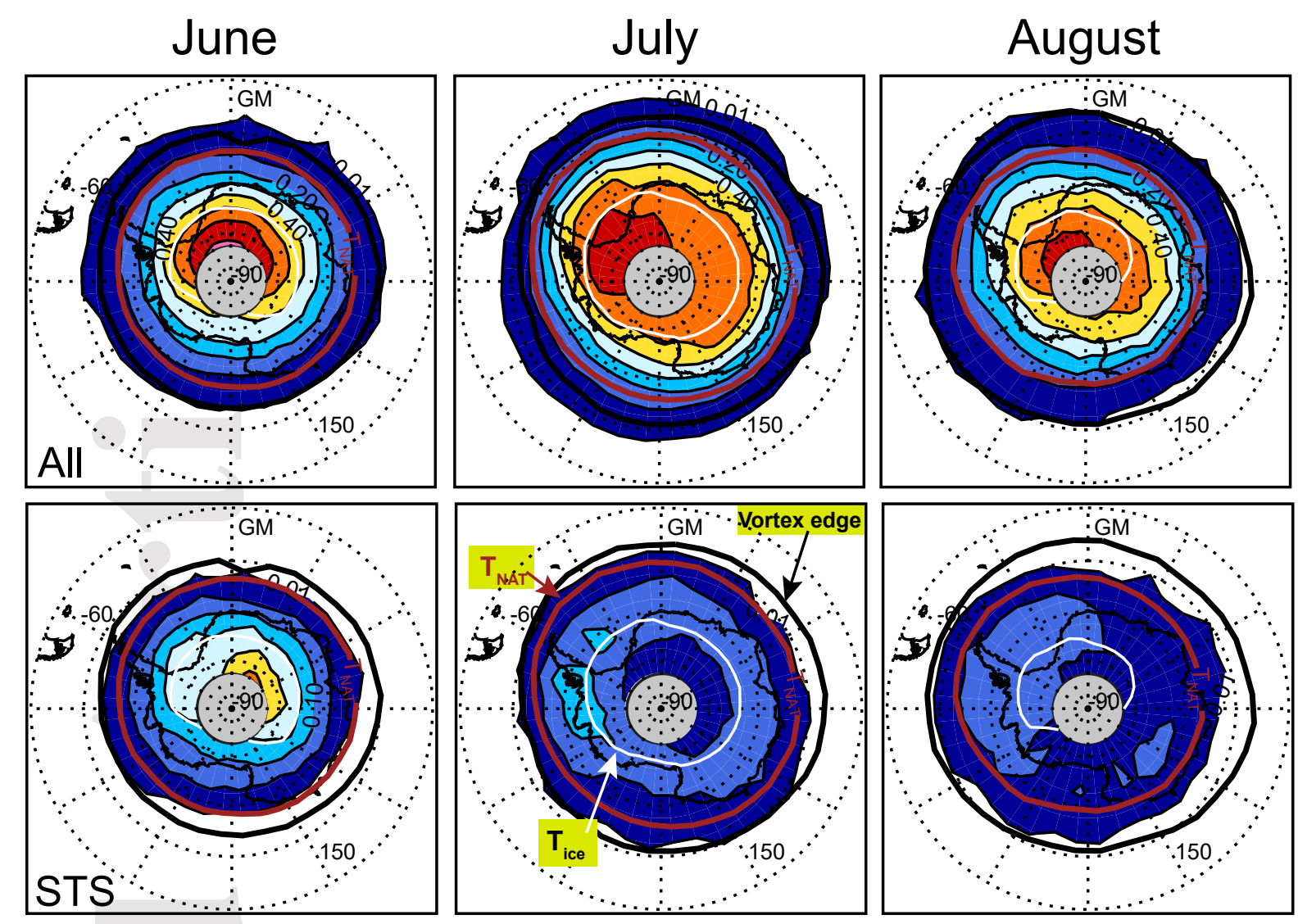

\section{September}
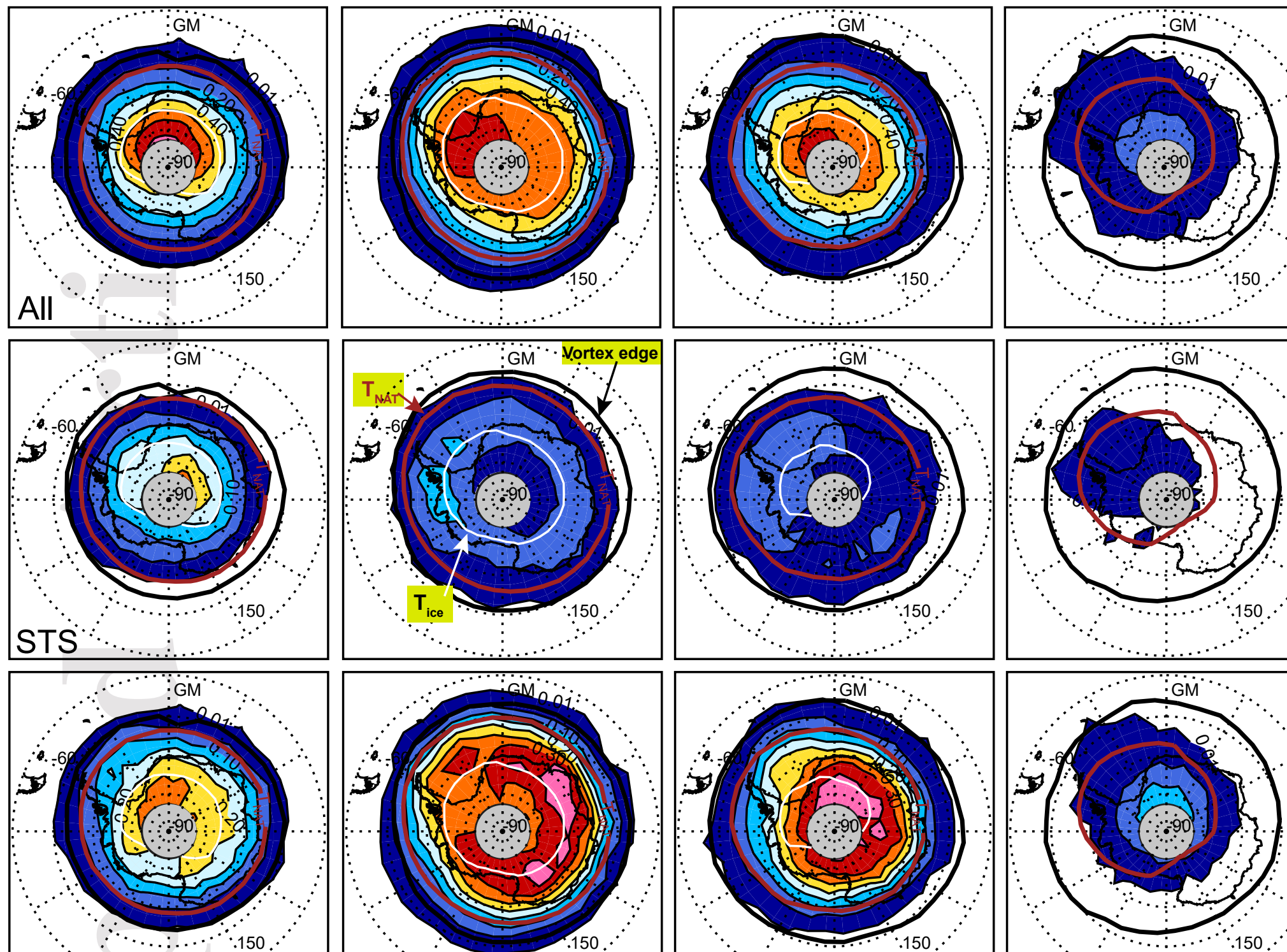

NAT M M
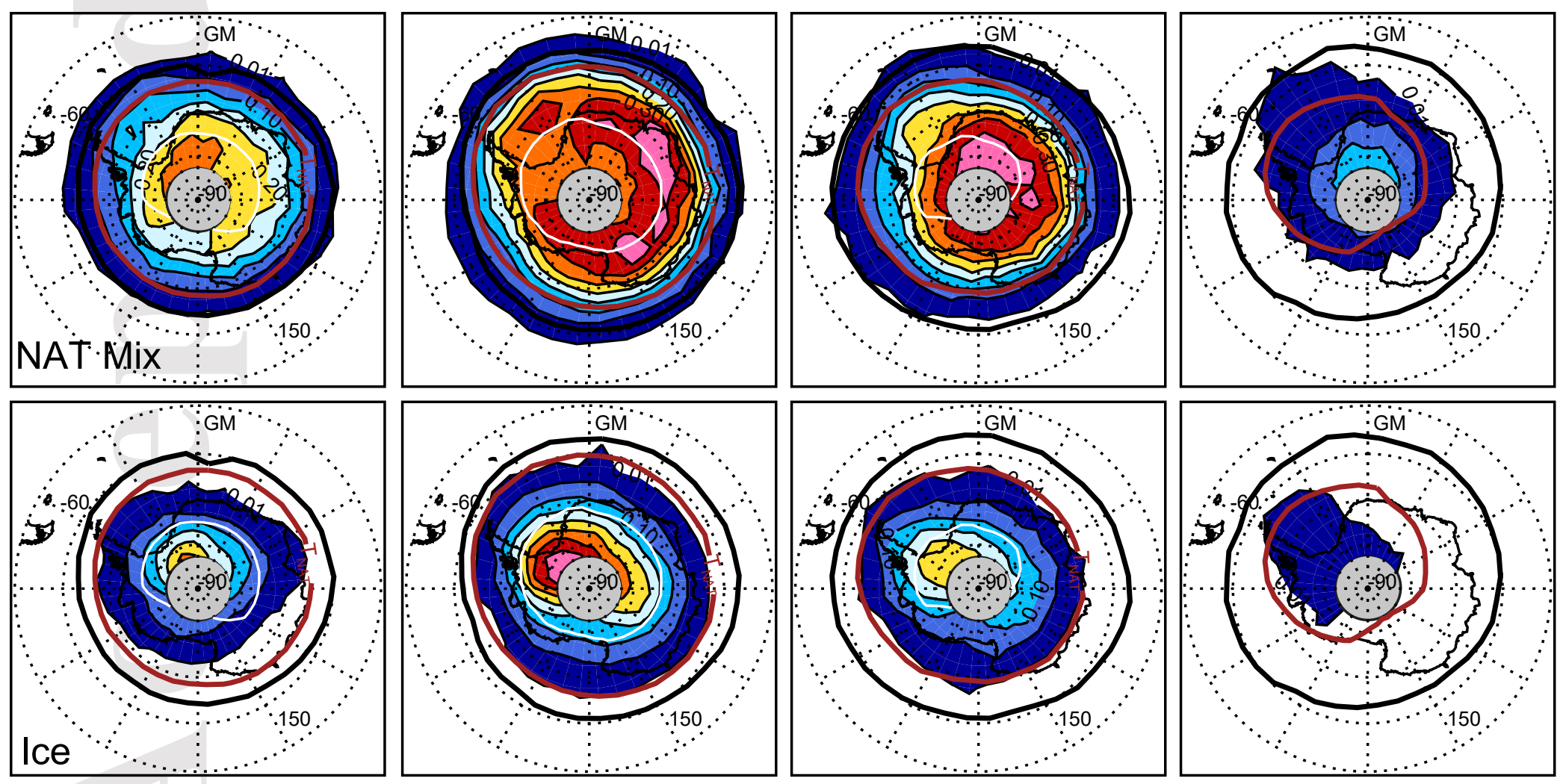
0.01
0.1
0.2
0.3
0.5
0.6
0.7
All
0.01
$0.1 \quad 0.15$
0.2
0.25
0.3
0.35
Individual
This article is protected Bysfyrgicaurrenterererequency 


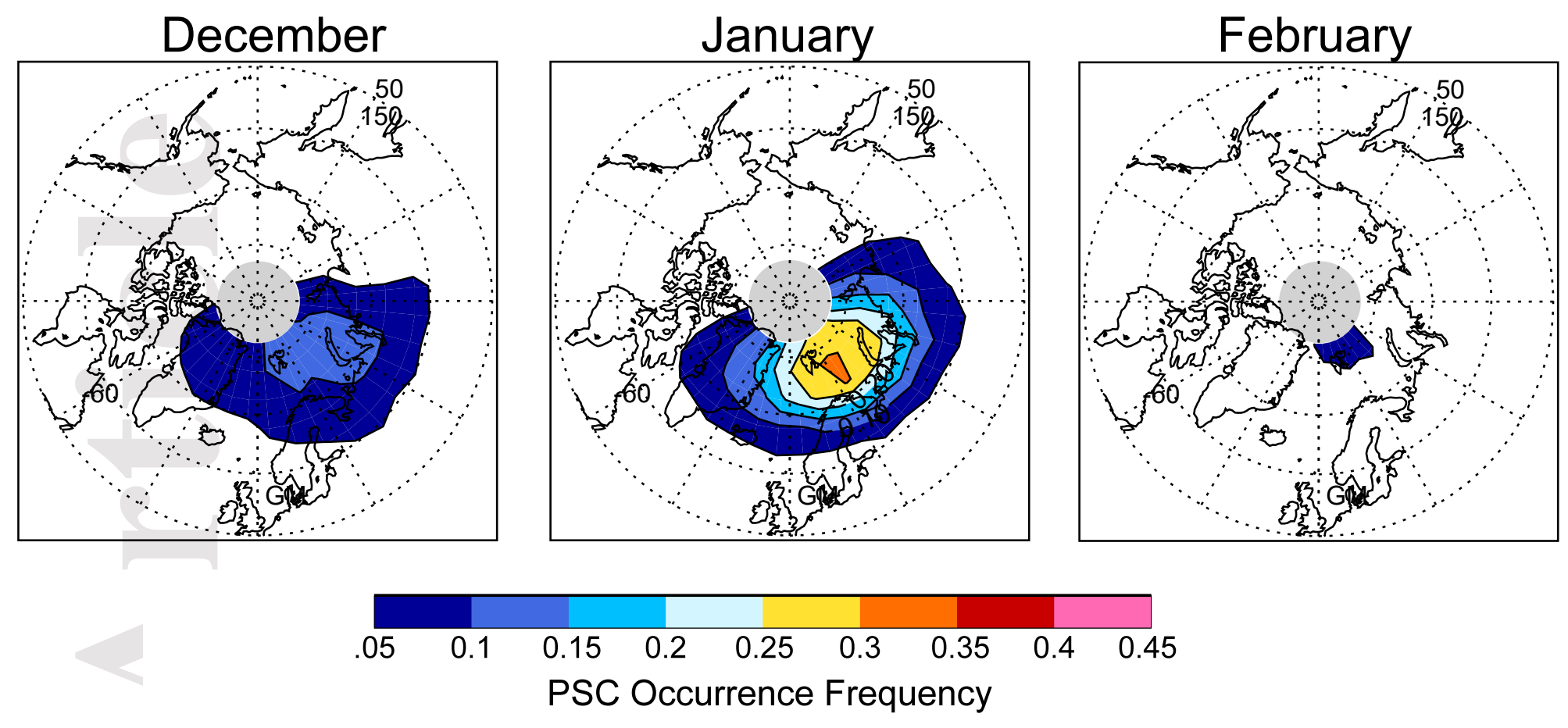

This article is protected by copyright. All rights reserved. 
Кวuәnbəد」

난 00000000

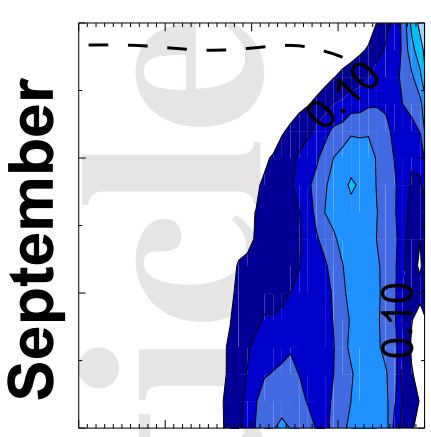

$\frac{\sqrt{3}}{3}$
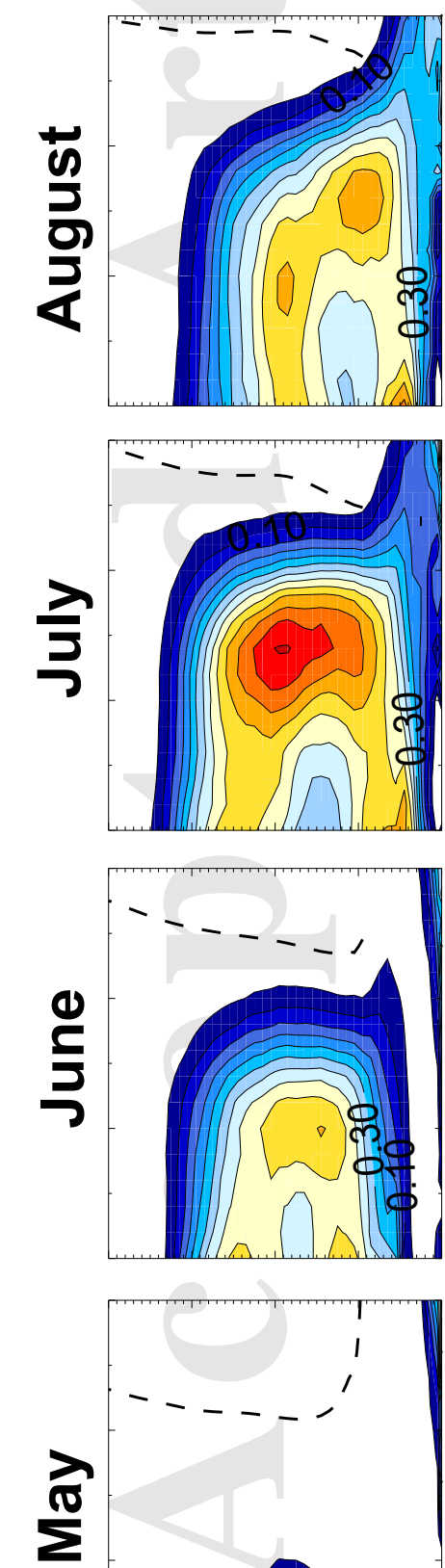

( $\wedge$ qdd) $\varepsilon O N H$

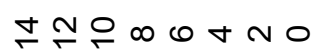
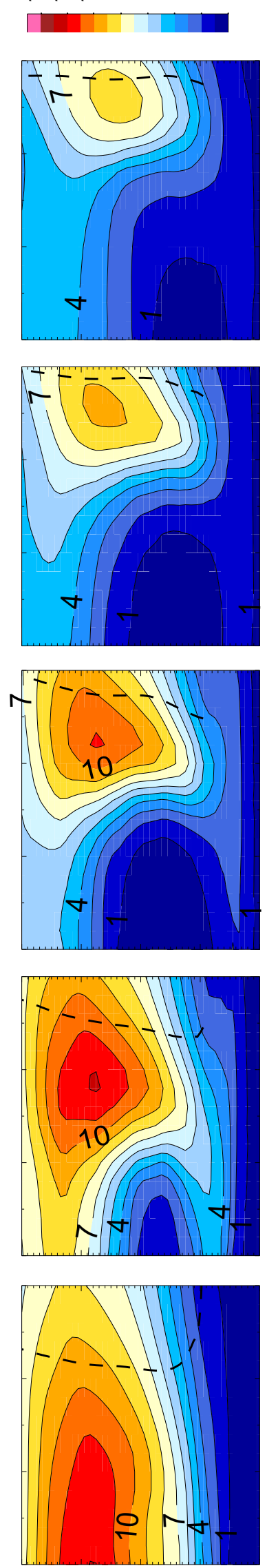

(^udd) $\mathrm{OZH}$

$\infty \wedge \bullet$ เ $\rightarrow$ N
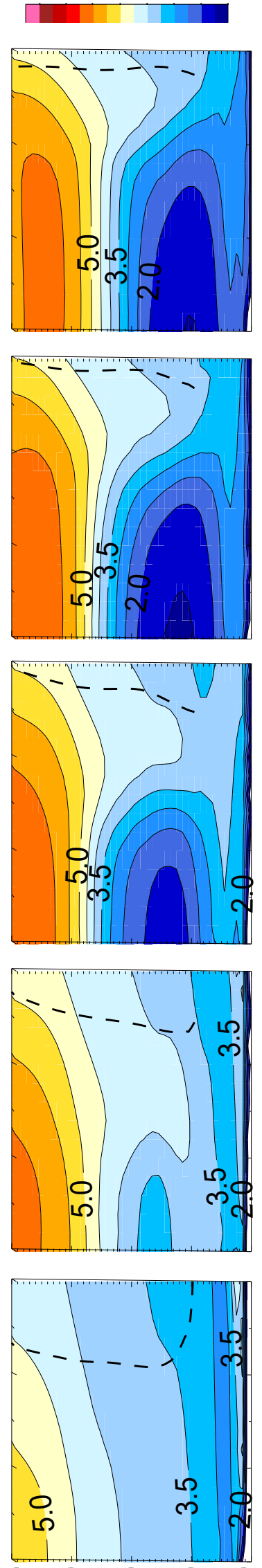

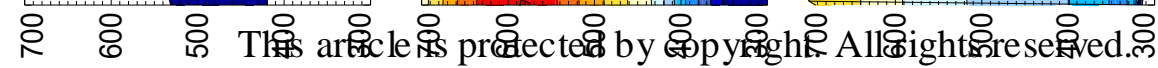
duə। ןe!̣uəłod (y) $\perp$

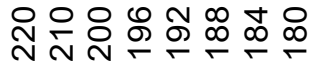

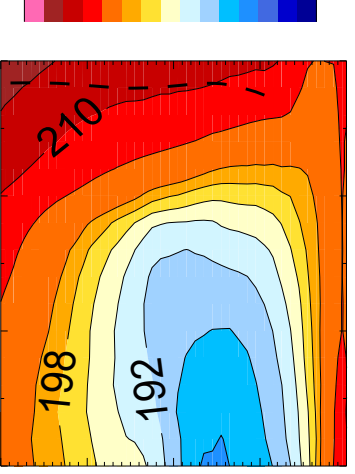

윤ㅁำ
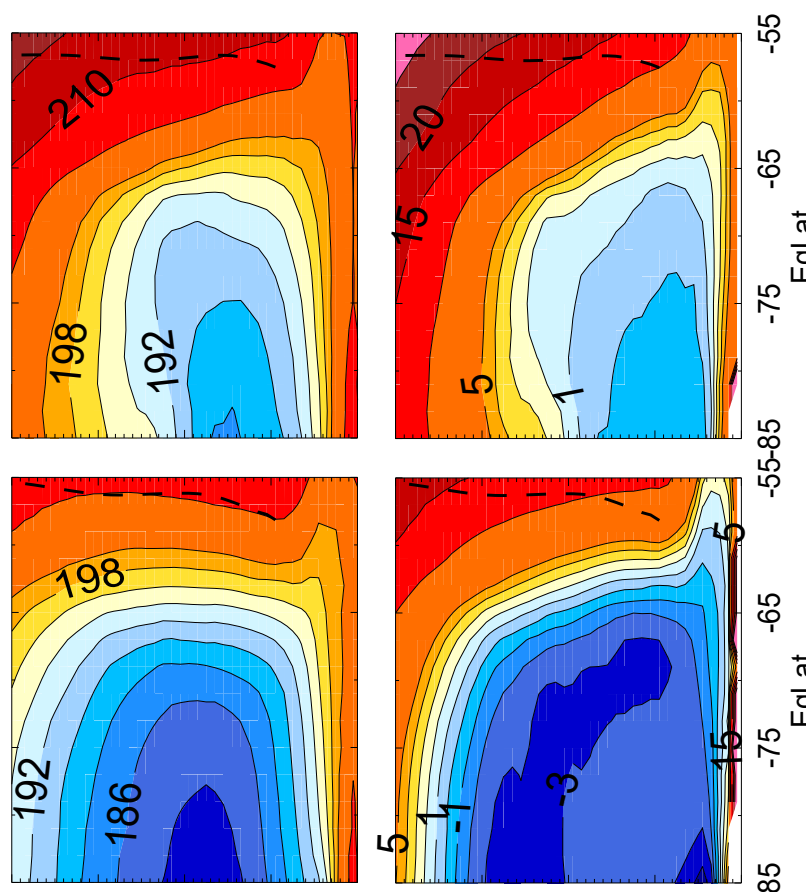

$\infty$

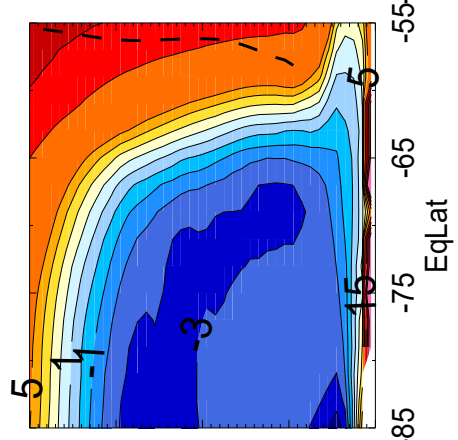

$\infty$
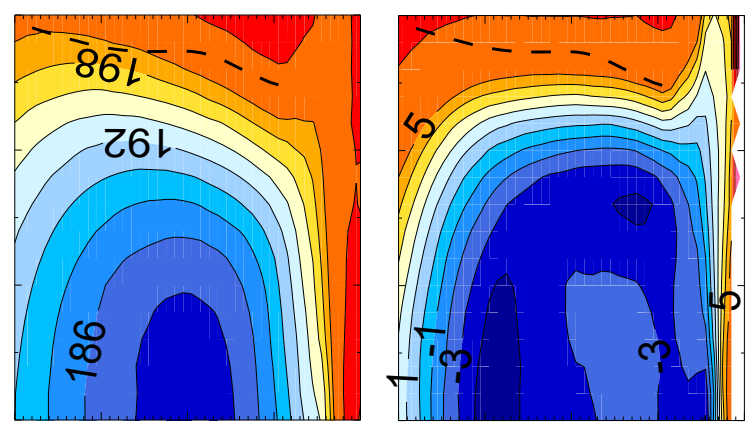

畧
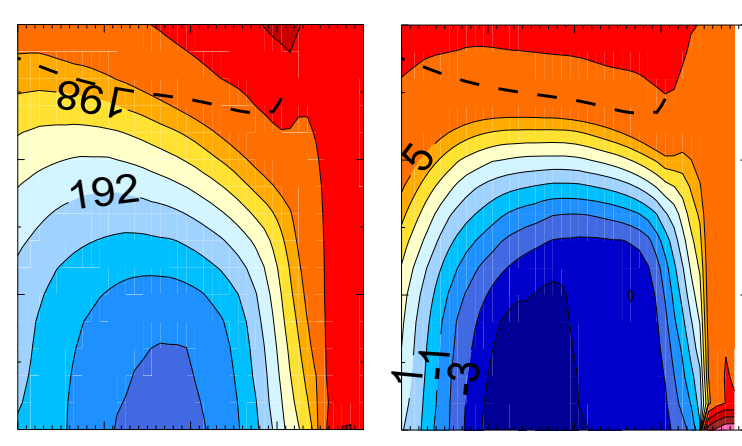

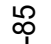

นุ

@ొ

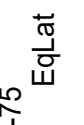

$\stackrel{1}{\infty}$

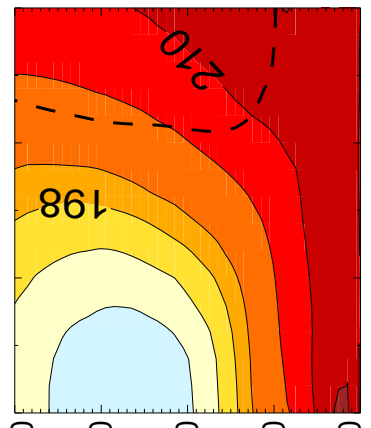

ஓ \& \& \& \&

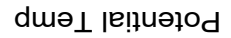

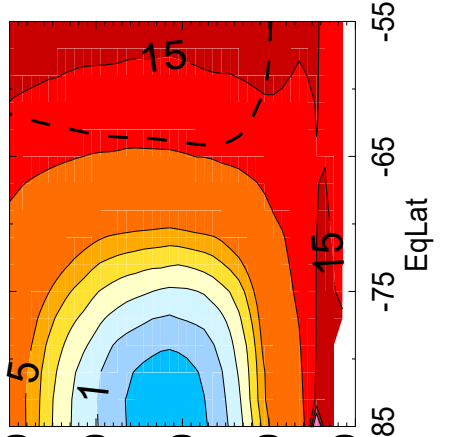

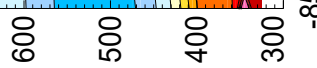

duәр |е!̨uәғо 

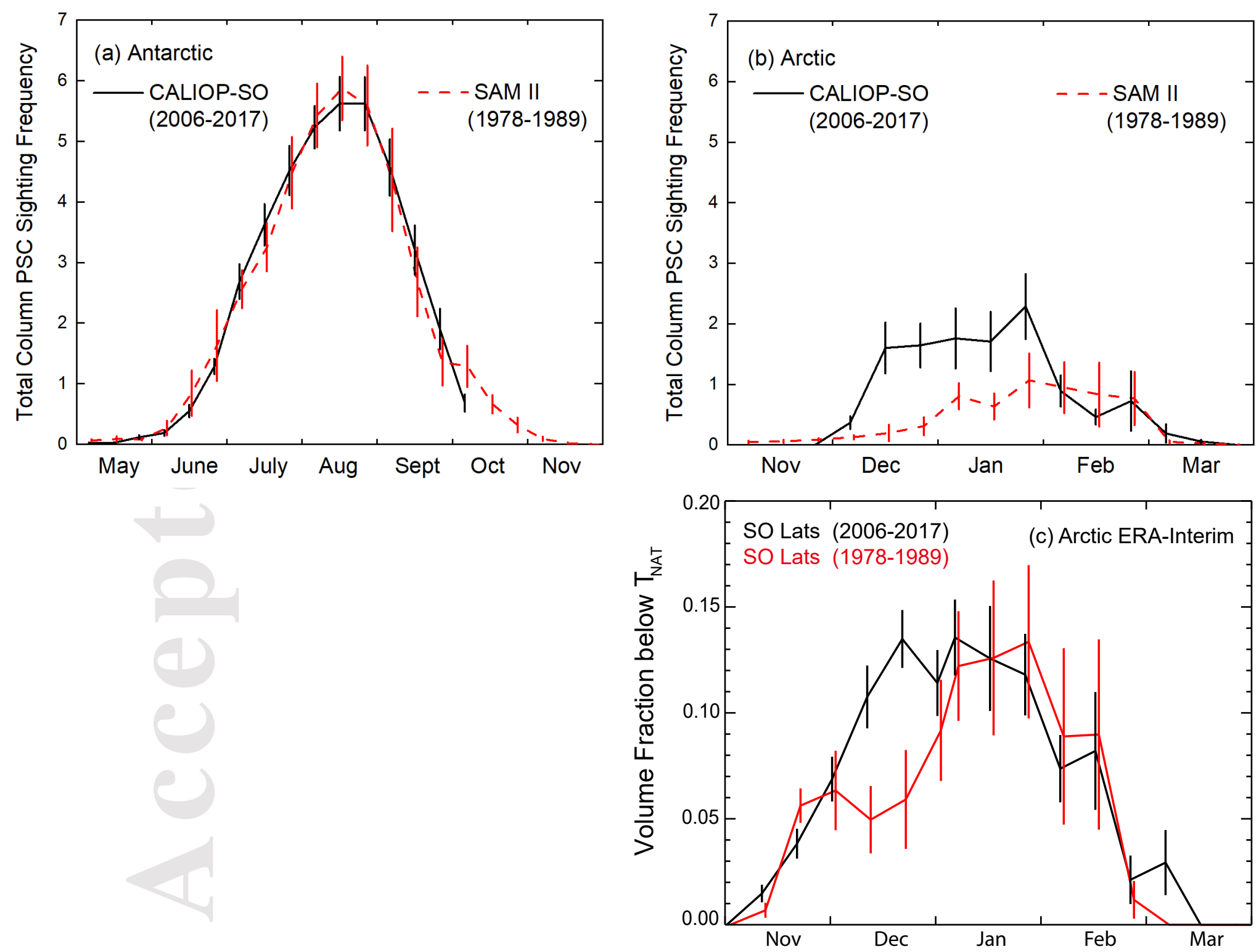

This article is protected by copyright. All rights reserved. 

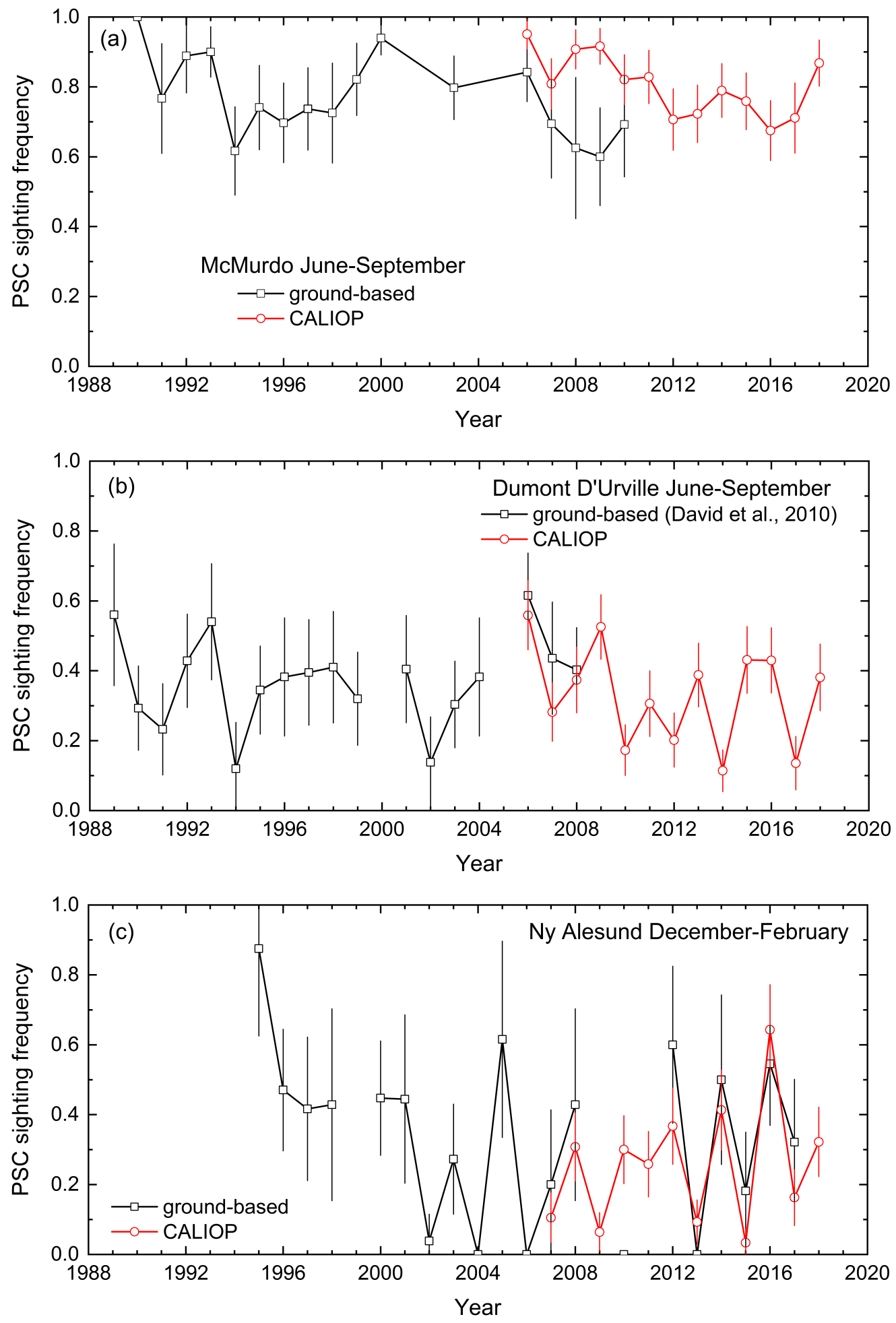

This article is protected by copyright. All rights reserved. 


\section{$\mathrm{H}_{2} \mathrm{SO}_{4} / \mathrm{H}_{2} \mathrm{O}$}

$T[\mathrm{~K}] \quad$ liquid stratospheric sulfuric acid aerosol

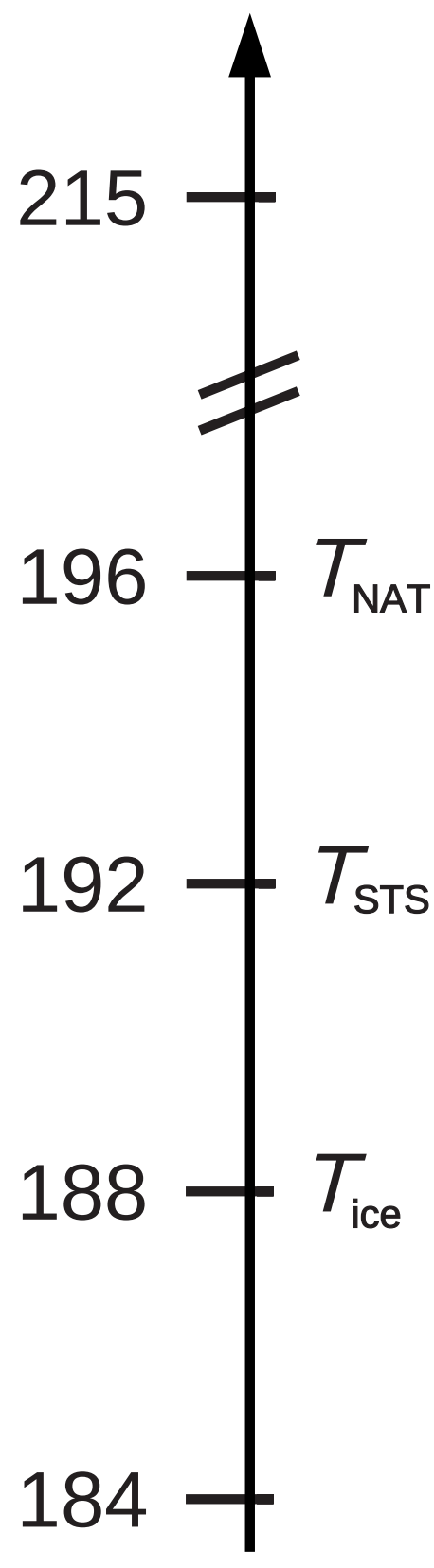
SSA

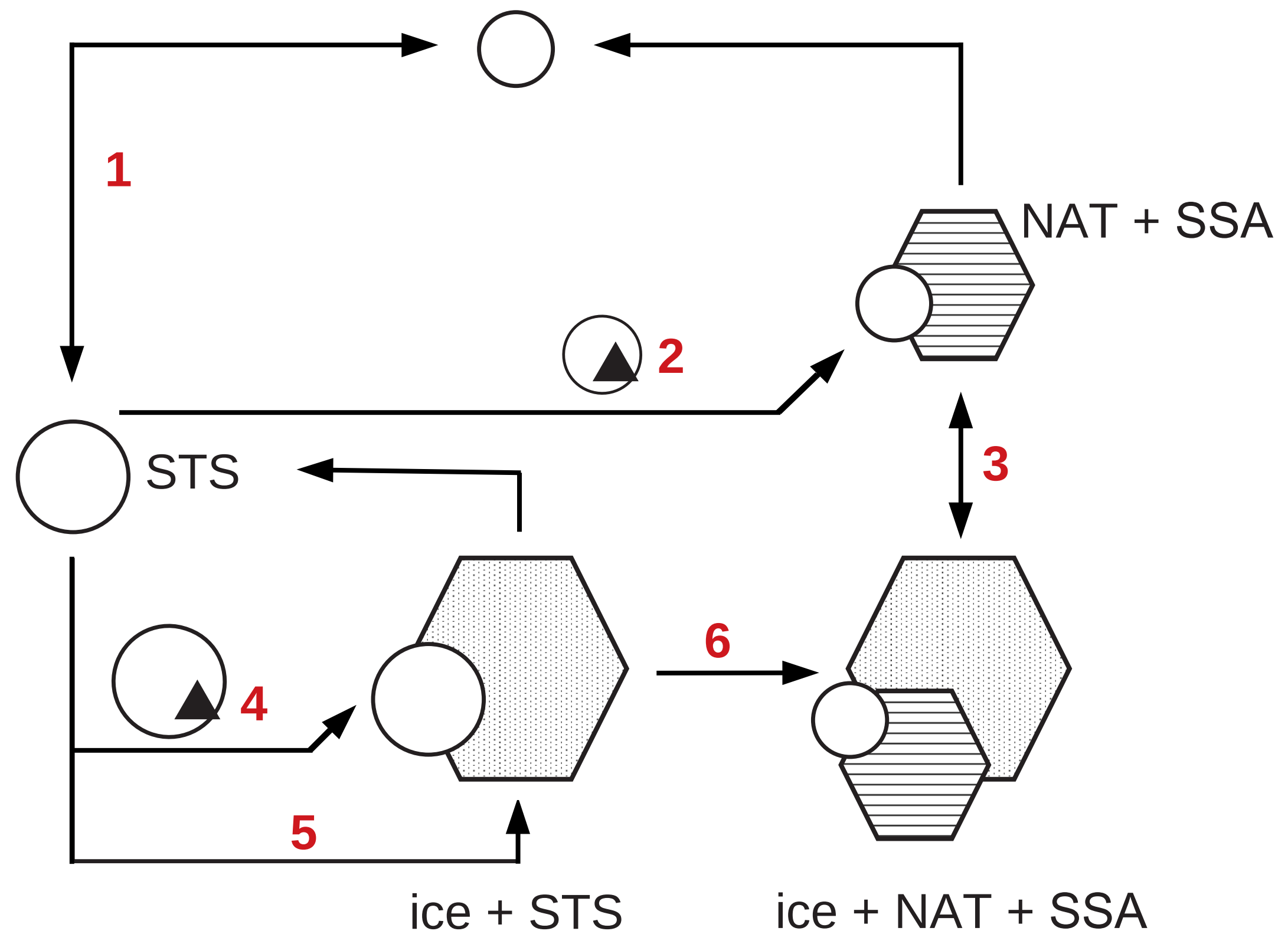



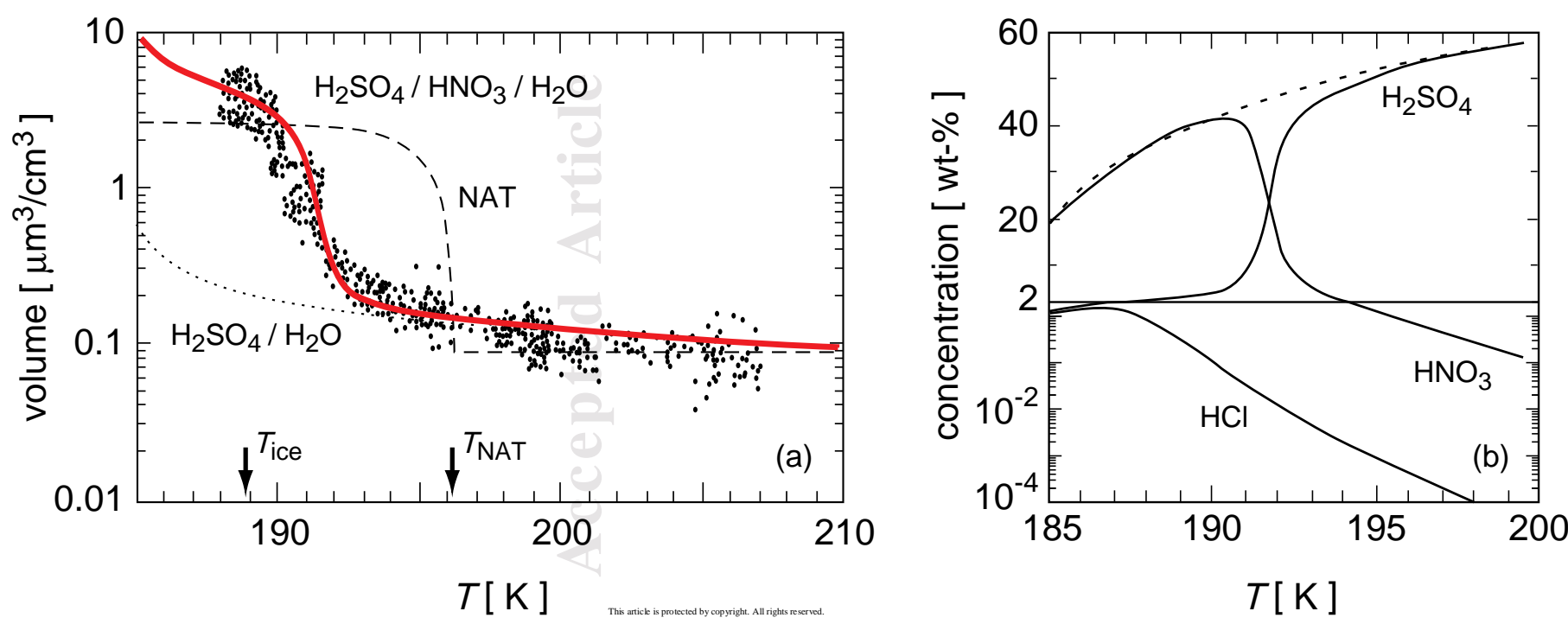

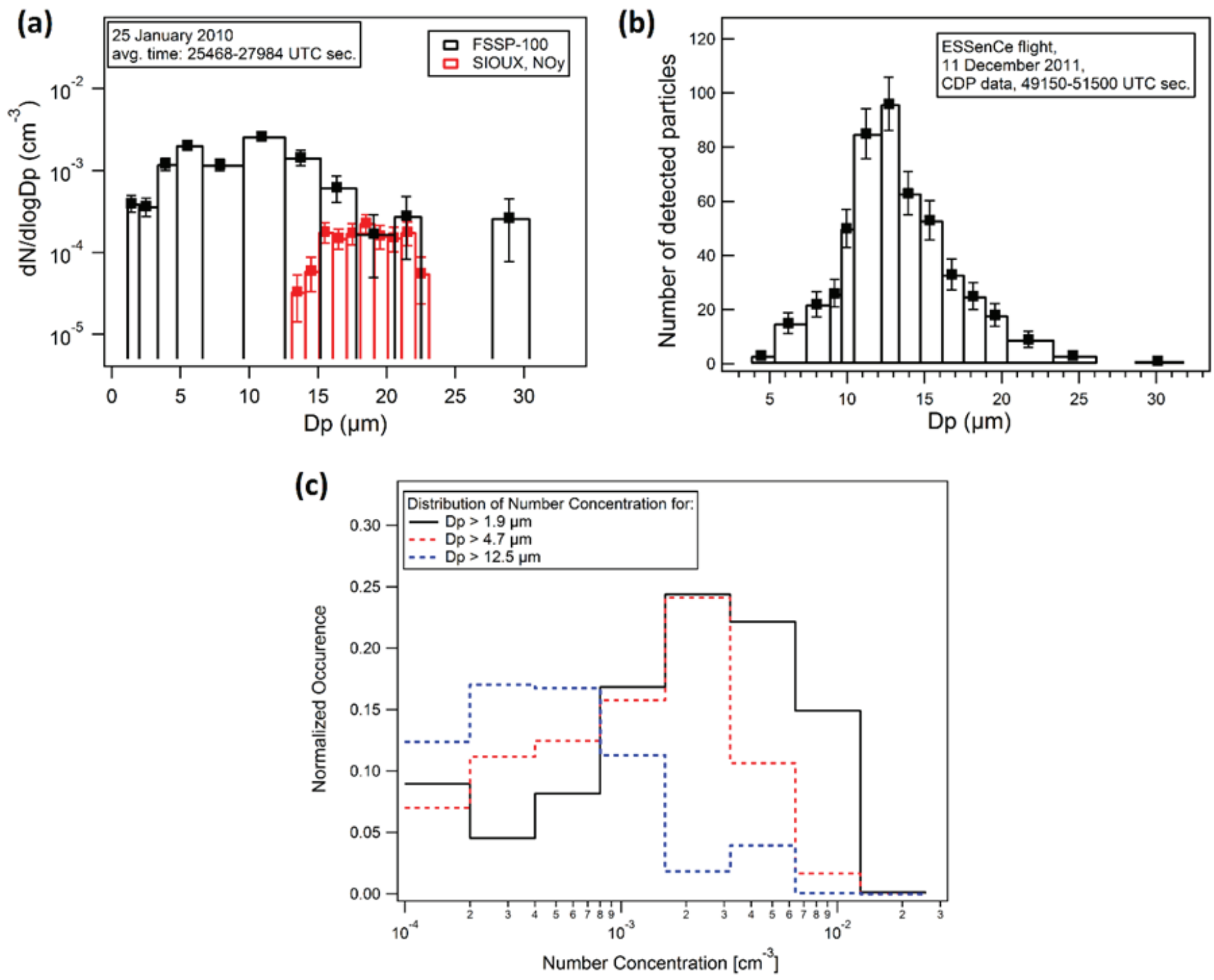

This article is protected by copyright. All rights reserved. 

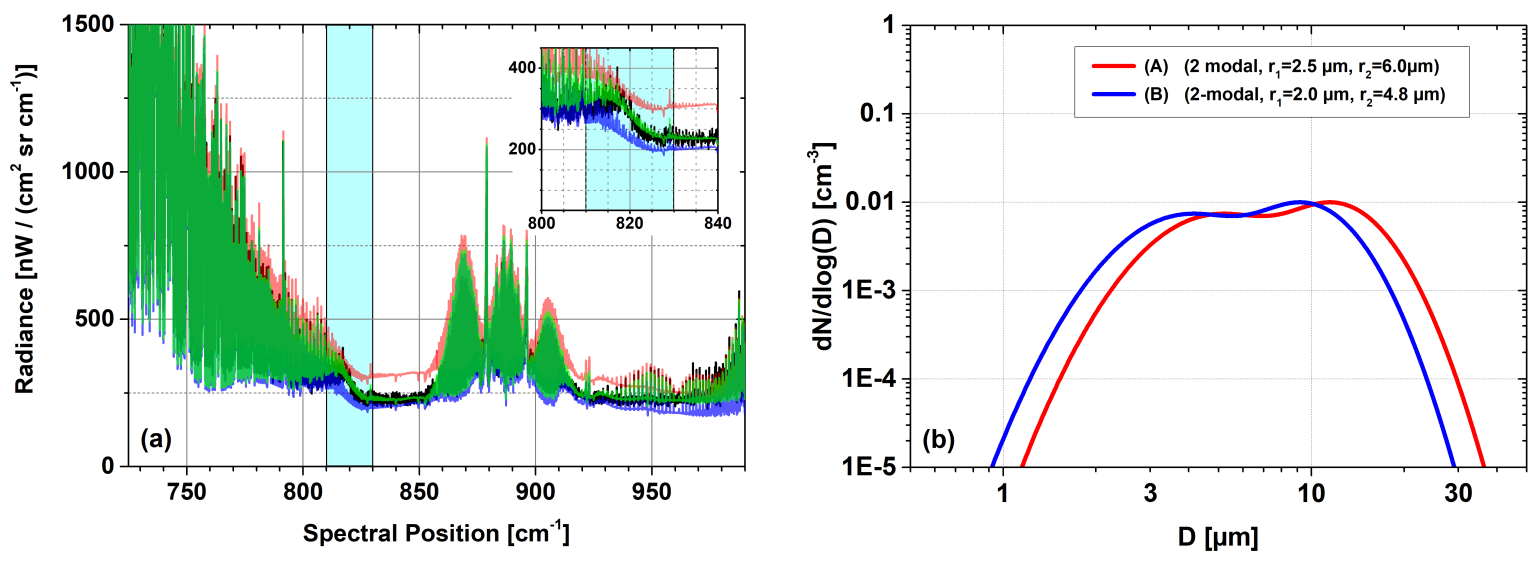

This article is protected by copyright. All rights reserved. 


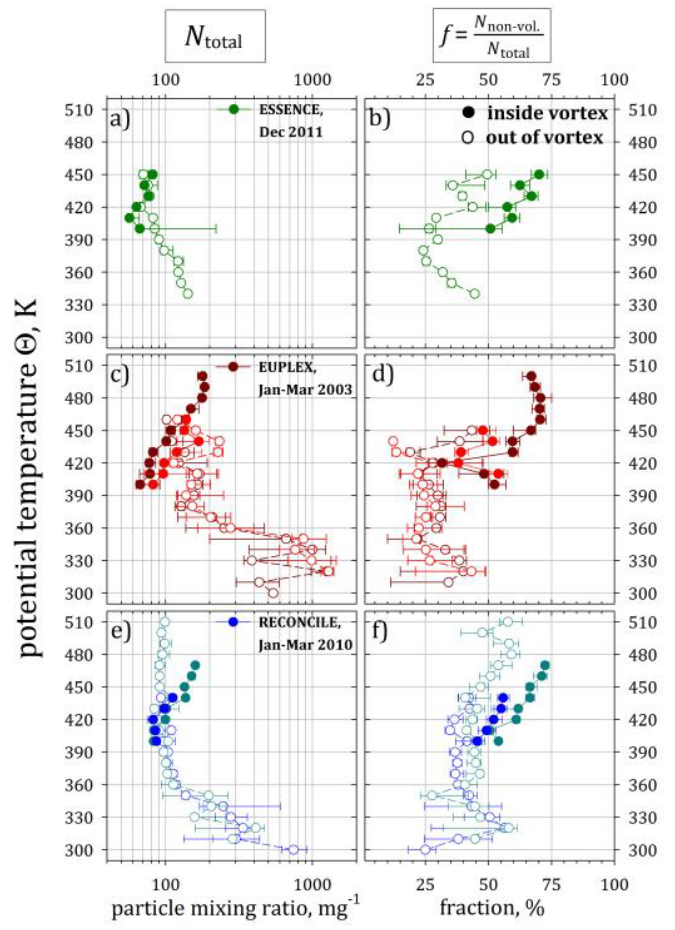

This article is protected by copyright. All rights reserved. 


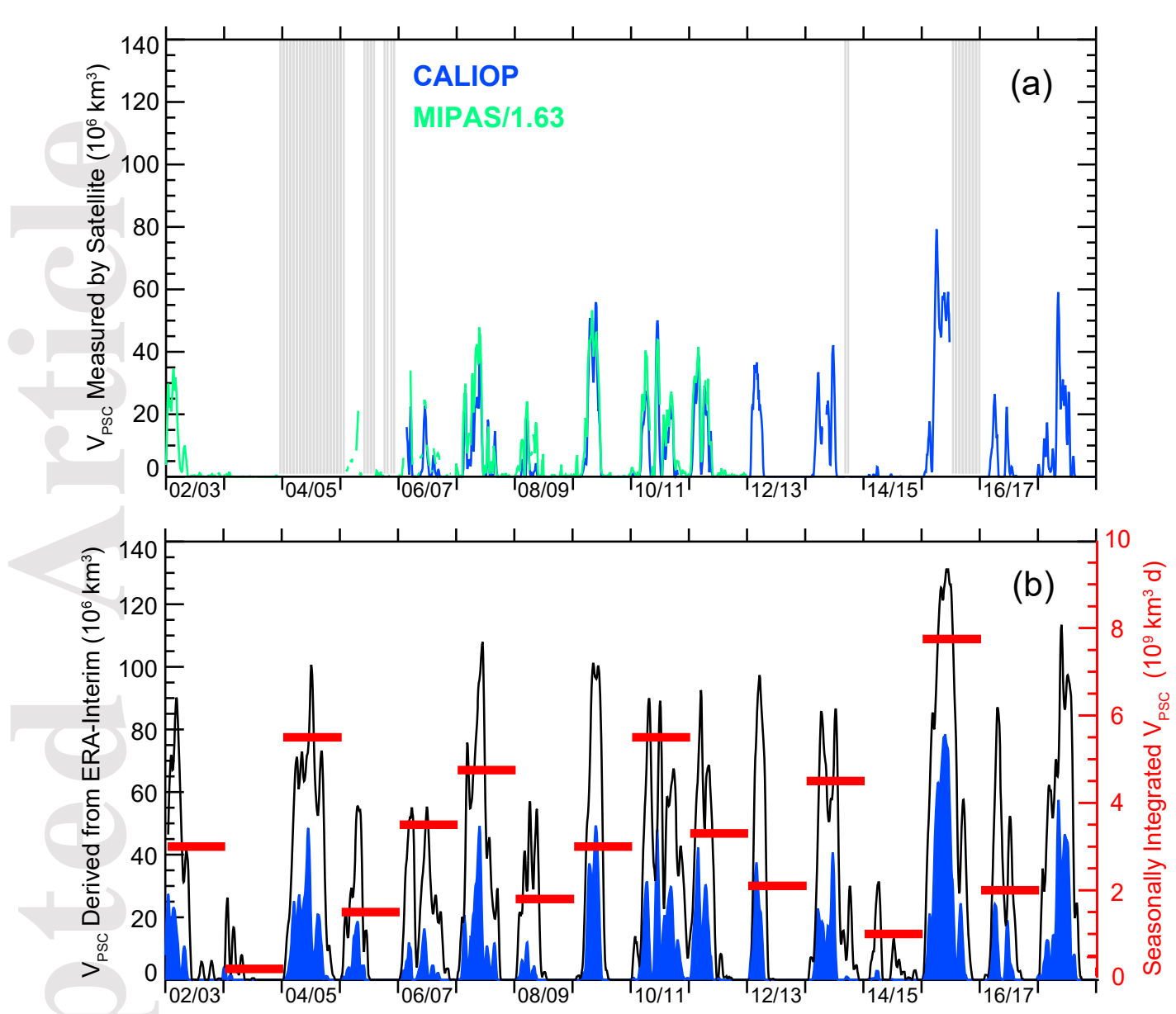

This article is protected by copyright. All rights reserved. 

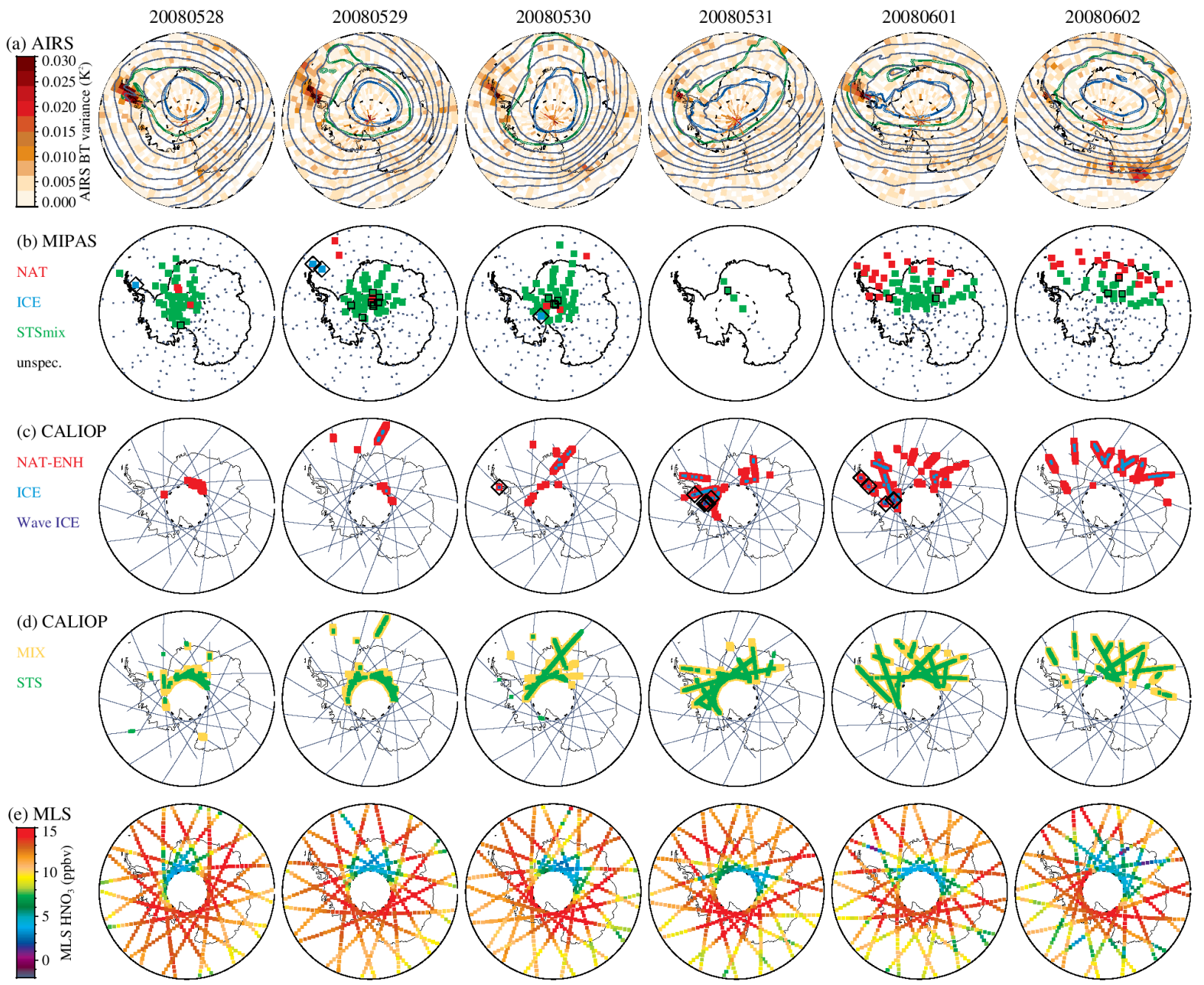

This article is protected by copyright. All rights reserved. 


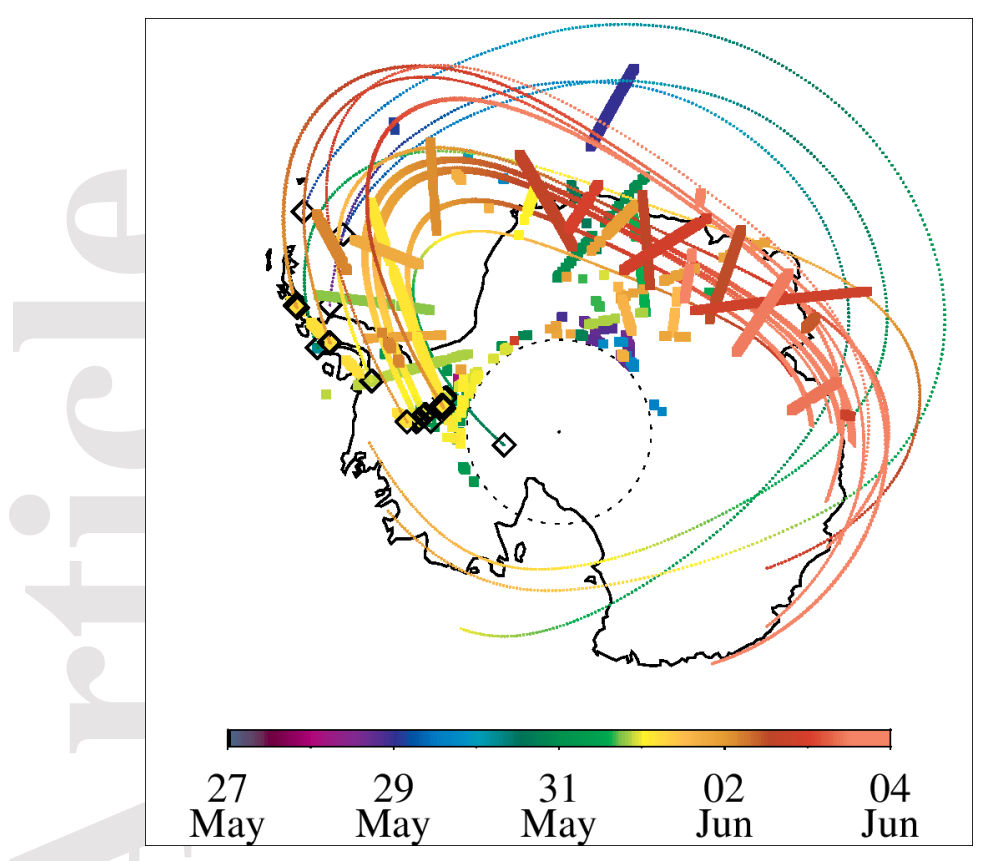

This article is protected by copyright. All rights reserved. 

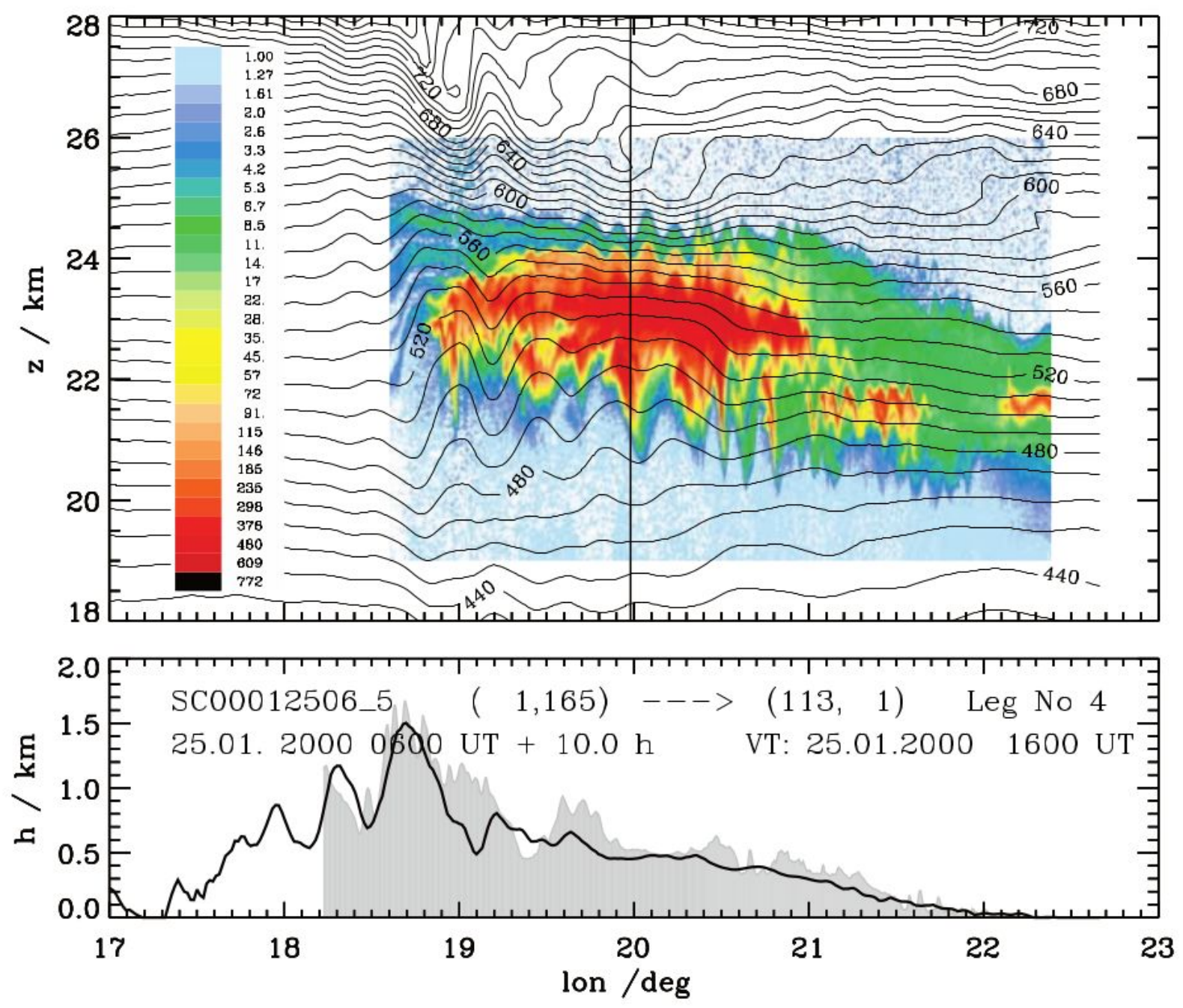

This article is protected by copyright. All rights reserved. 

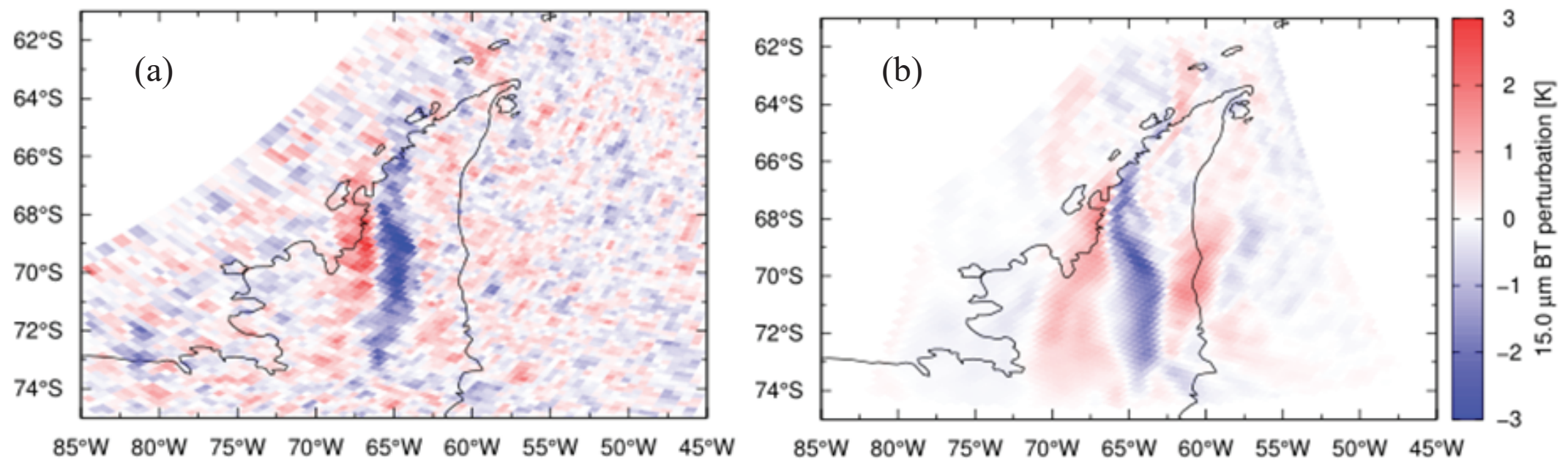
Large ozone depletion in late winter (15 September 2008) at 18-km altitude

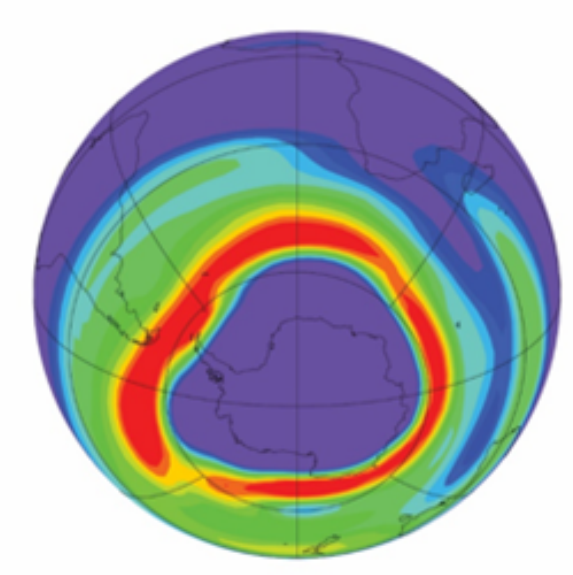

Ozone

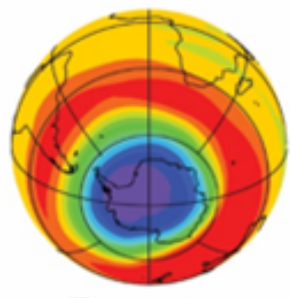

Temperature

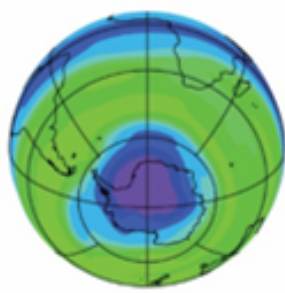

Hydrogen chloride ( $\mathrm{HCl})$

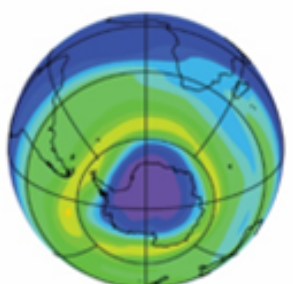

Nitric acid $\left(\mathrm{HNO}_{3}\right)$

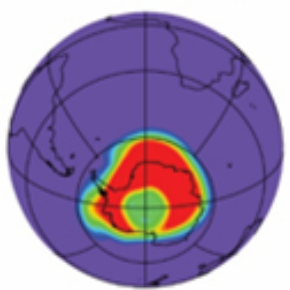

Chlorine monoxide (ClO)

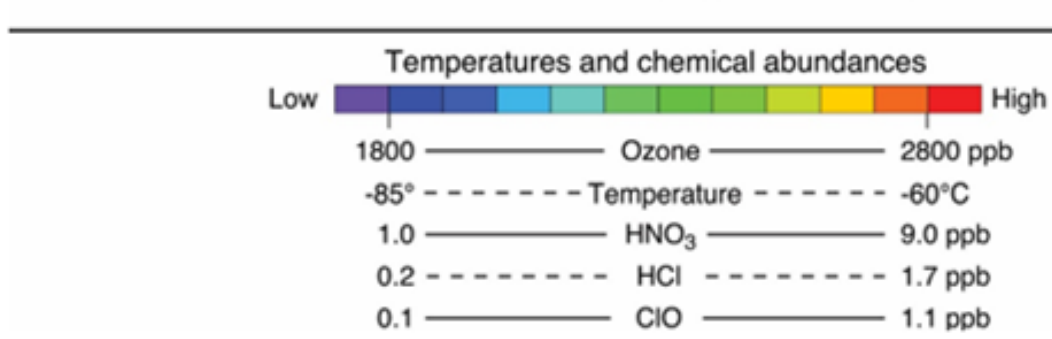

This article is protected by copyright. All rights reserved. 

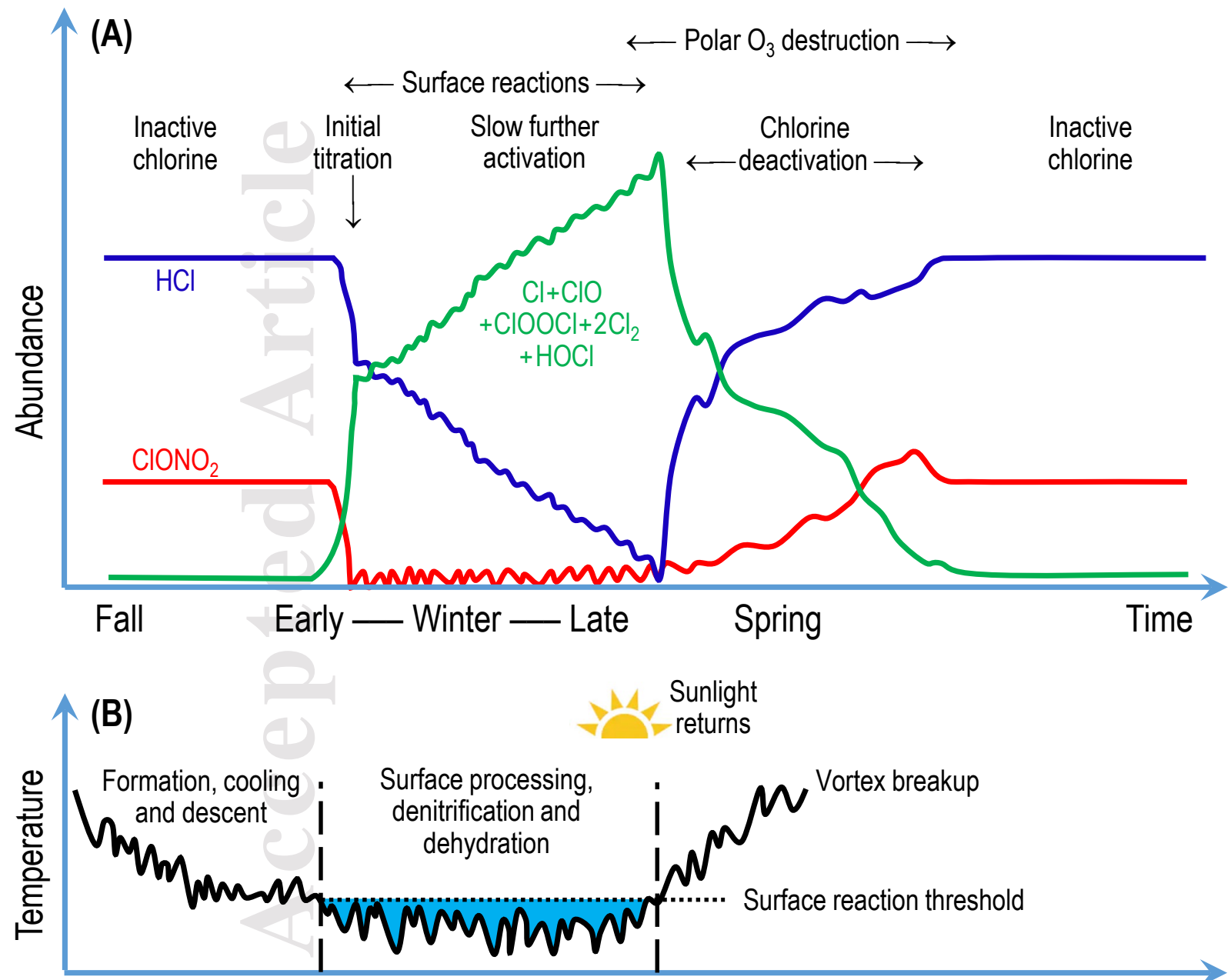

Polar vortex evolution 
$\mathrm{H}_{2} \mathrm{SO}_{4} \mathrm{wt} \%$

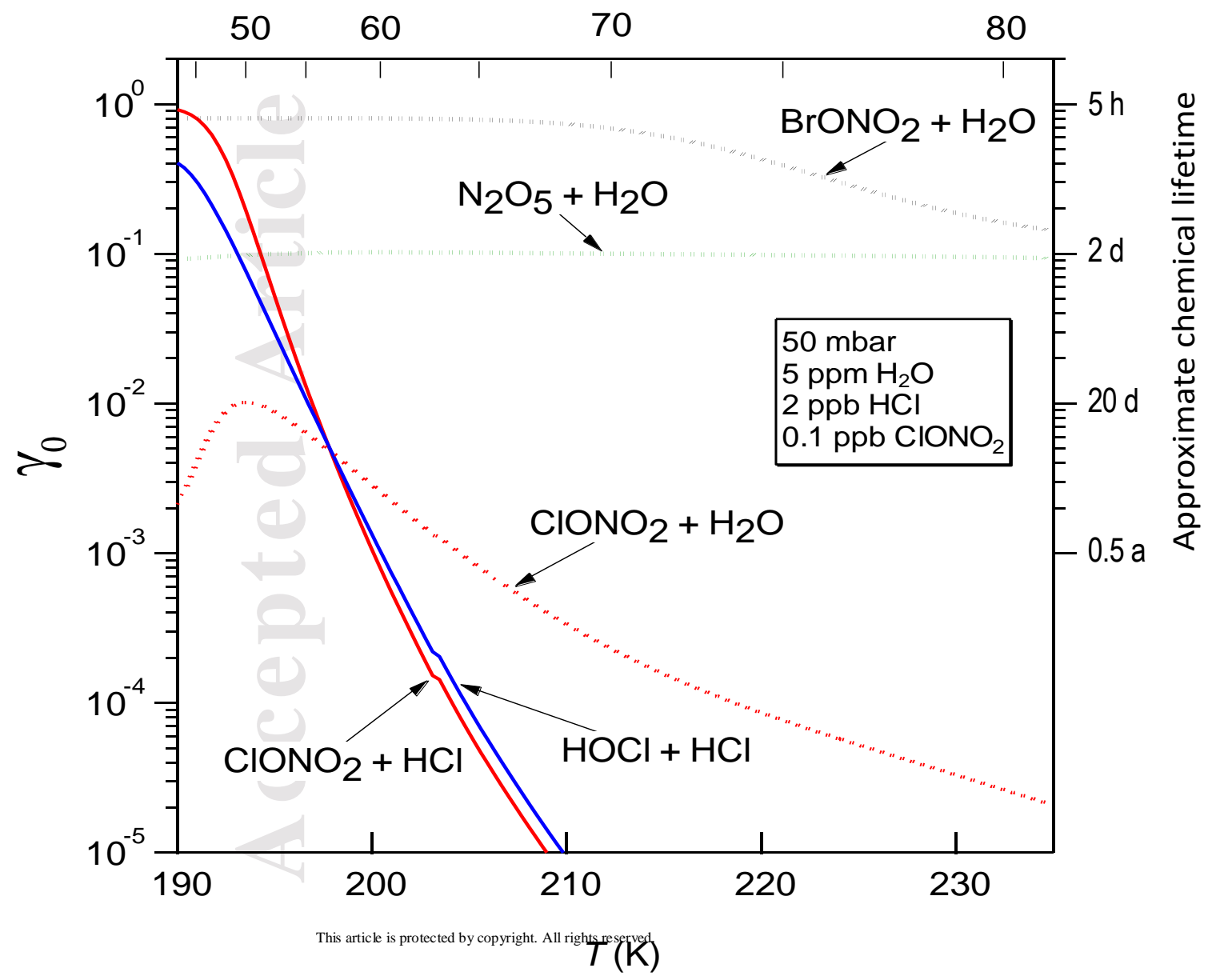




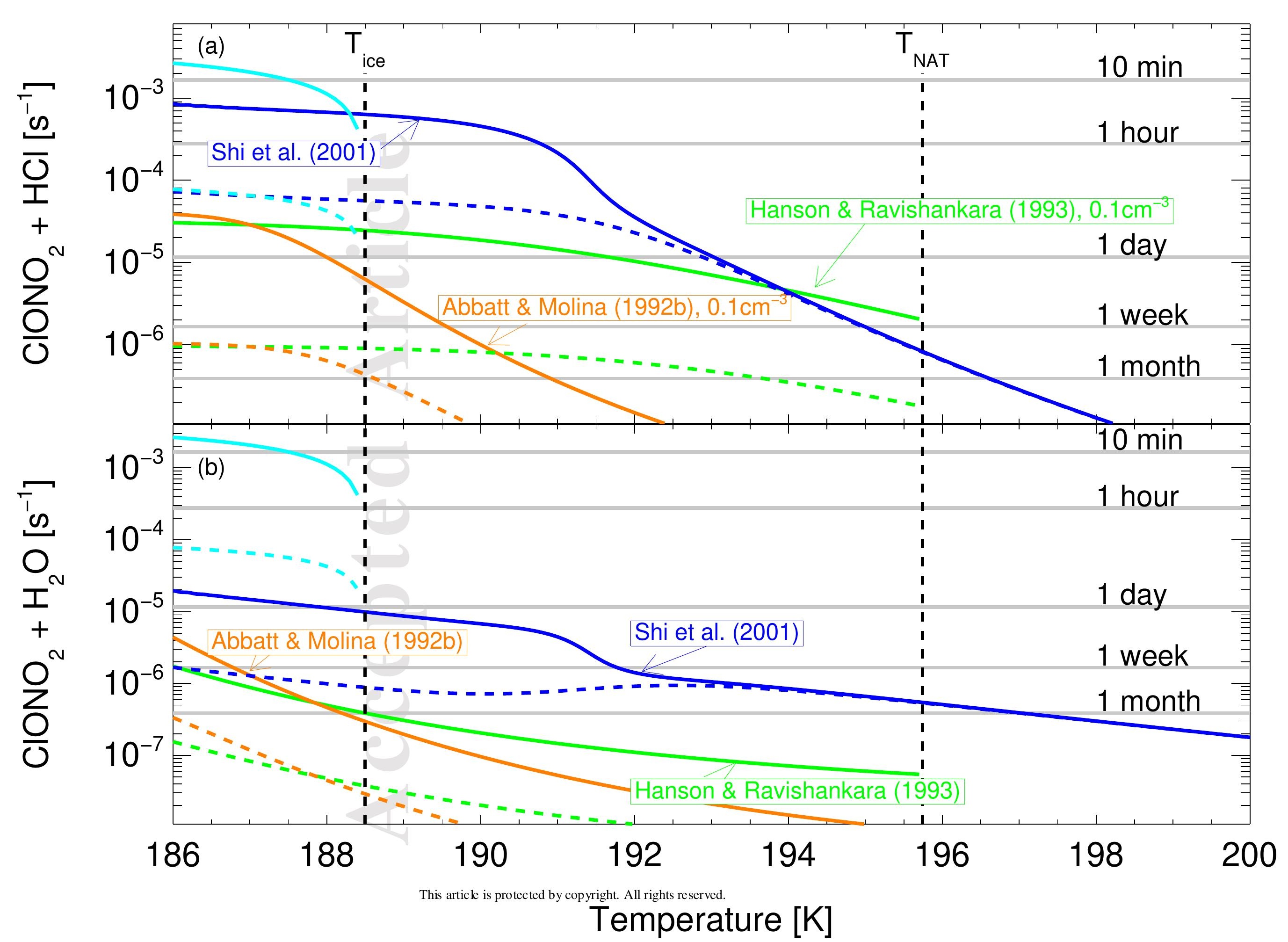




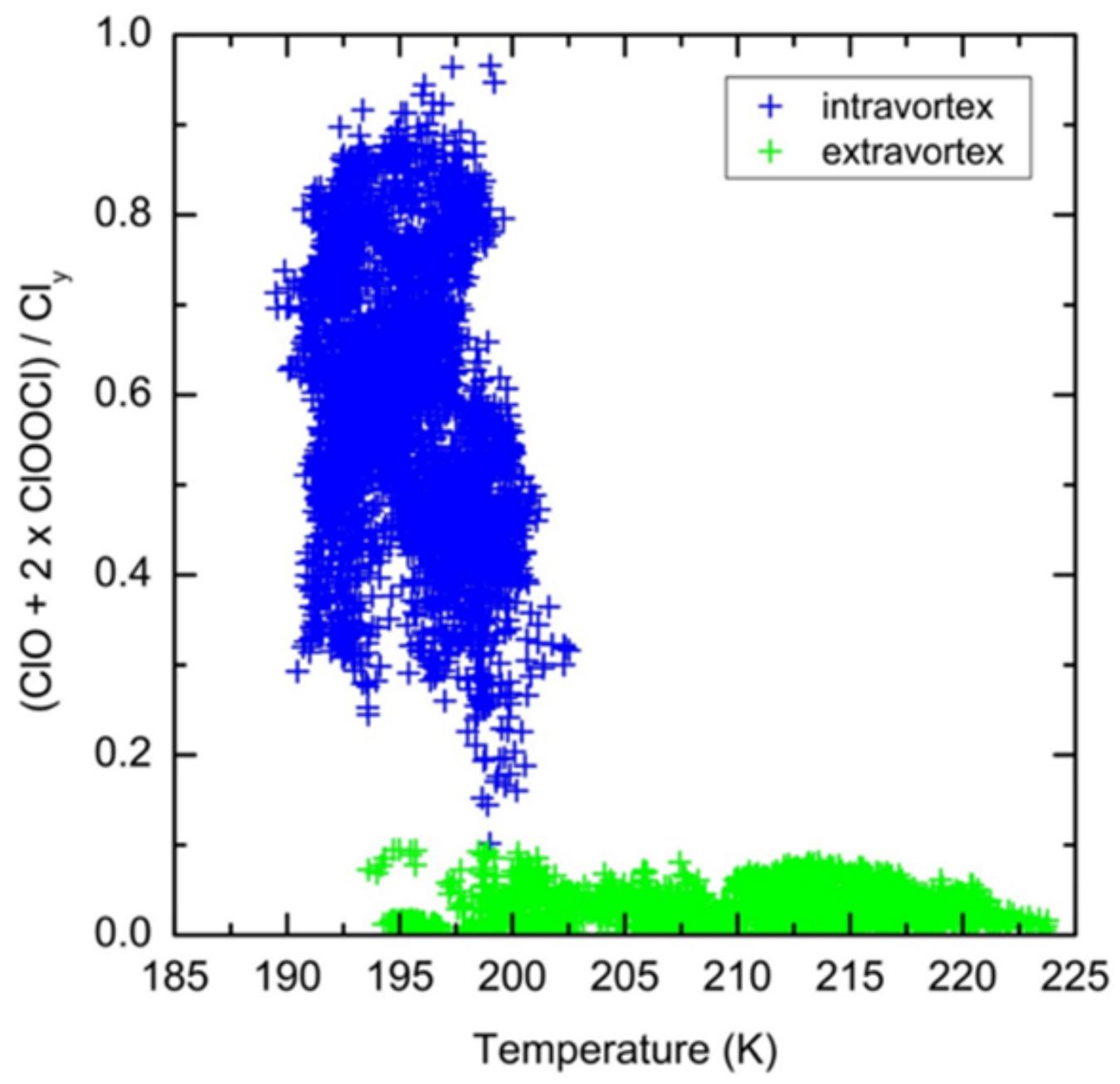

This article is protected by copyright. All rights reserved. 


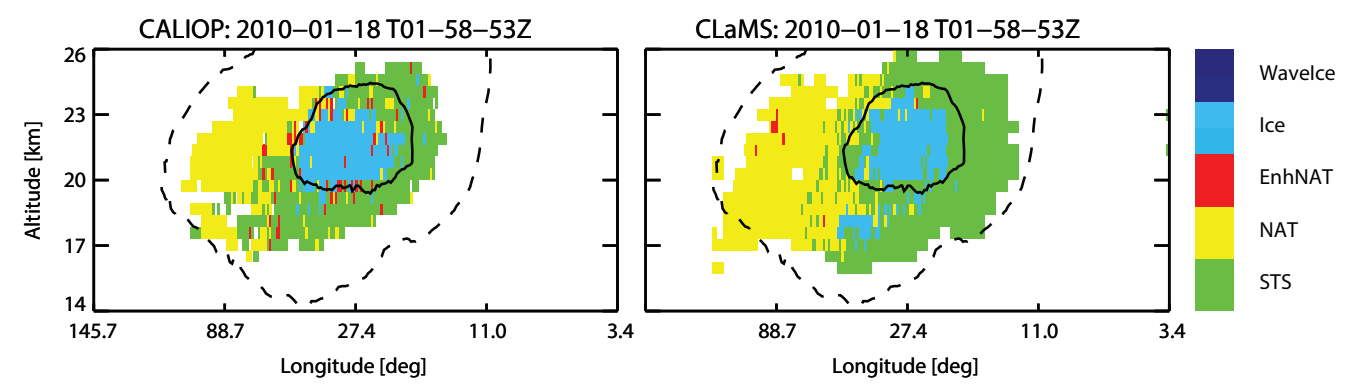

This article is protected by copyright. All rights reserved. 
July
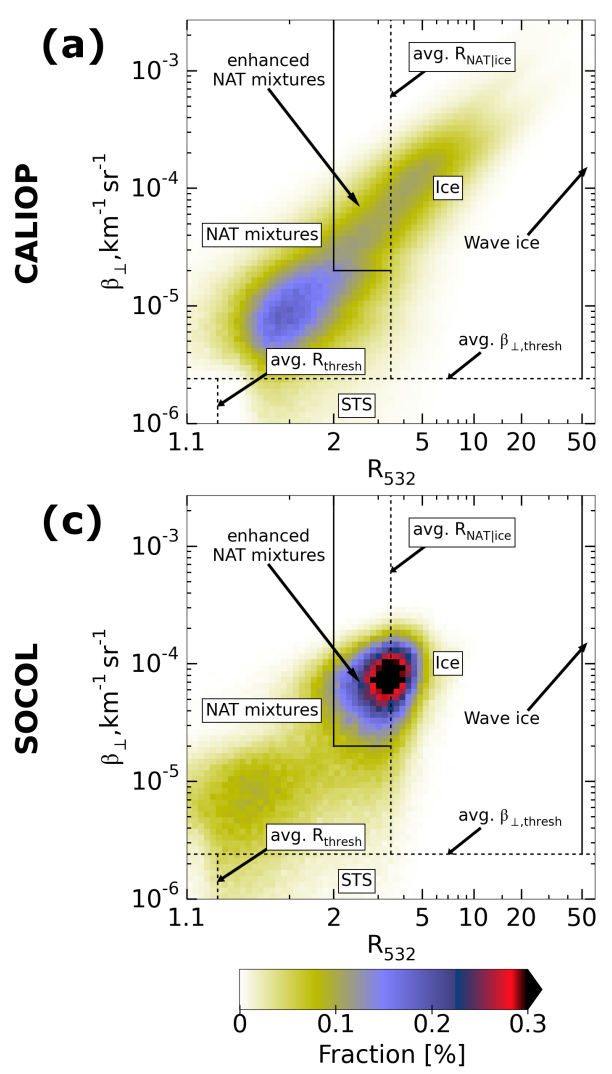

May - October
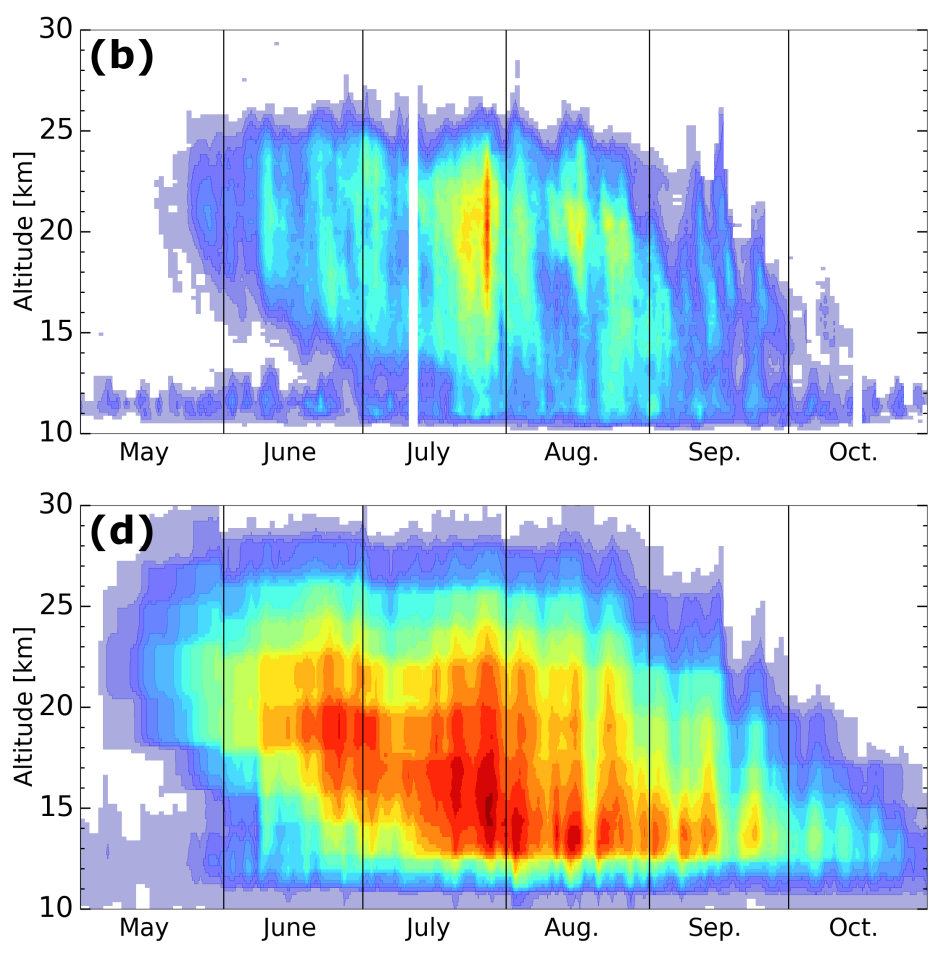

This article is protected by copyright. All rights reserved. 

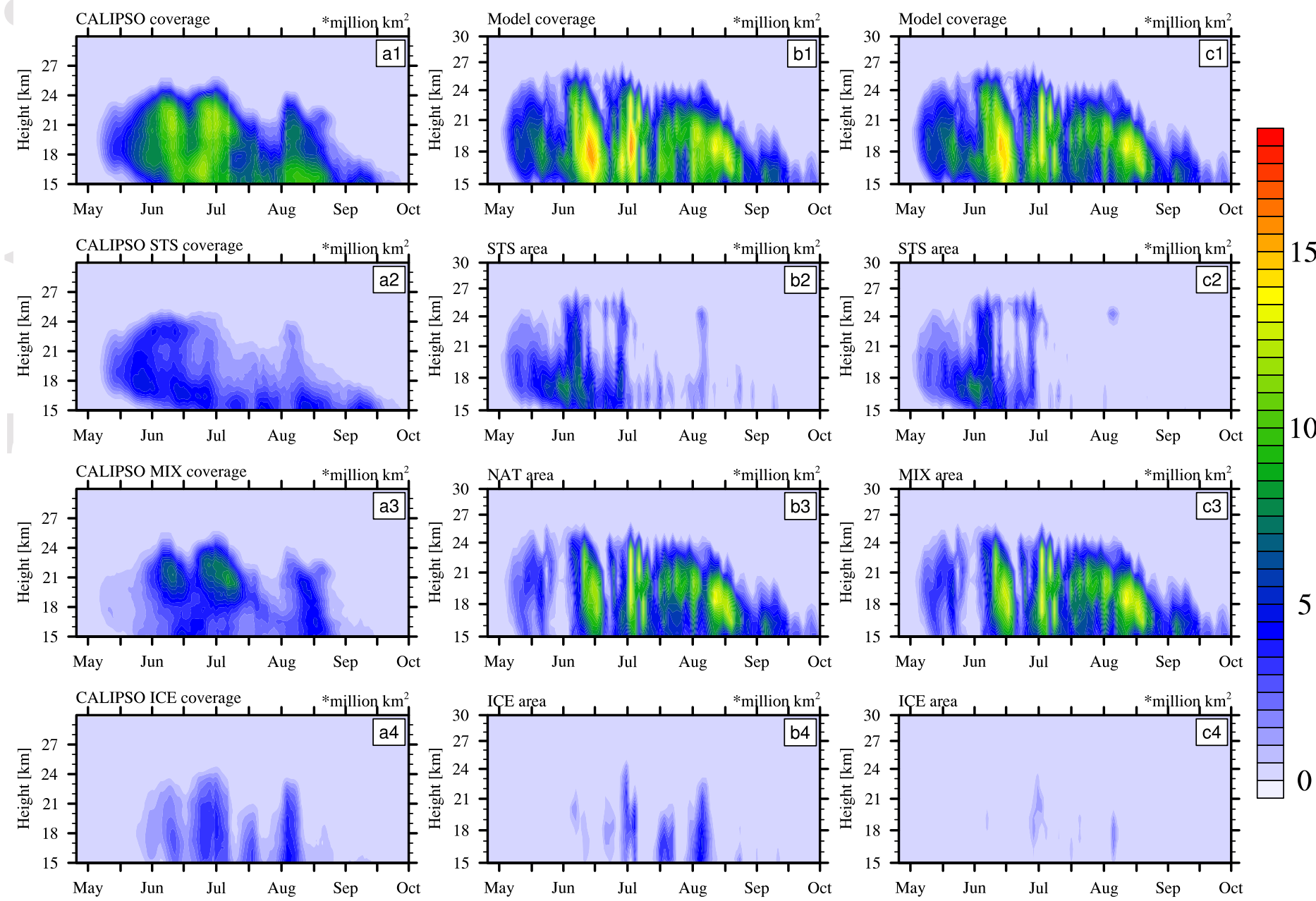

This article is protected by copyright. All rights reserved. 